\title{
Dynamic Walking Models to Understand Asymmetric Gait Characteristics
}

Charles, William G.

How to cite:

Charles, William G. (2019) Dynamic Walking Models to Understand Asymmetric Gait Characteristics. Doctoral thesis, Swansea University.

http://cronfa.swan.ac.uk/Record/cronfa48133

Use policy:

This item is brought to you by Swansea University. Any person downloading material is agreeing to abide by the terms of the repository licence: copies of full text items may be used or reproduced in any format or medium, without prior permission for personal research or study, educational or non-commercial purposes only. The copyright for any work remains with the original author unless otherwise specified. The full-text must not be sold in any format or medium without the formal permission of the copyright holder. Permission for multiple reproductions should be obtained from the original author.

Authors are personally responsible for adhering to copyright and publisher restrictions when uploading content to the repository.

Please link to the metadata record in the Swansea University repository, Cronfa (link given in the citation reference above.)

http://www.swansea.ac.uk/library/researchsupport/ris-support/ 


\section{Dynamic Walking Models to Understand Asymmetric Gait Characteristics}

\section{William George Charles}

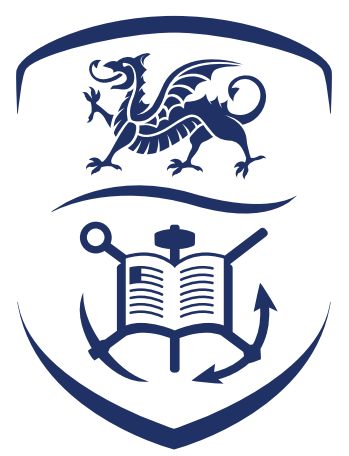

\section{Swansea University Prifysgol Abertawe}

Submitted to Swansea University in fulfilment of the requirements for the Degree of

Doctor of Philosophy 


\title{
Dynamic Walking Models to Understand Asymmetric Gait Characteristics
}

\author{
by \\ William George Charles
}

\begin{abstract}
Passive dynamic walking models remarkably predict gait behaviour such as walk-run transition speeds, preferred step length, stride frequencies and - with the inclusion of springs - ground reaction forces. Muscular or neurological conditions may lead to asymmetric walking characteristics that, in turn, come with long term health risks. Gait analysis may be used to understand an individual patient's conditions to help rehabilitate them. However, people adapt their kinematic and kinetic walking patterns so it can be hard to distinguish the effects of gait alterations such as inertial imbalance or injury. In this thesis a compass walking model with no active controllers is explored to understand the dynamics of gait. To help us interpret the effects of mass imbalance with a prosthetic foot or orthotic device, asymmetric loading conditions are investigated. A simple spring-mass walking model is used to explore the effects of altered touch-down angles and effective leg stiffness to see if these are used as strategies to alter the characteristics of gait. Results show that an asymmetric touchdown angle alters step length while retaining a symmetric stance time. A hybrid model is then derived with springs to emulate human-like ground reaction forces and asymmetric inertial loading of the legs. Results support previous research that pushoff from the trailing leg propels the leg mass more than the body mass. Higher rates of joint forces, larger step lengths and a longer stance time on the residual limb may be due to the prosthetic leg stiffness or the higher location of centre-of-mass. These results help us understand how the dynamic components affect gait characteristics such as step length, stance time and walking speeds. This work is motivated by the needs of persons with disabilities and by the desire to understand human walking.
\end{abstract}




\section{Declaration}

This work has not previously been accepted in substance for any degree and is not being concurrently

Signed

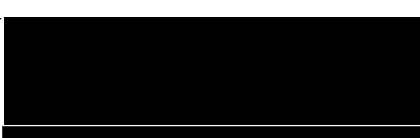

re for any degree.

Date

$01 / 11 / 18$

\section{Statement 1}

This thesis is the result of my own investigations, except where otherwise stated.

Other sources are acknowledged by footnotes giving explicit references. A bibliography is appended.

Signed

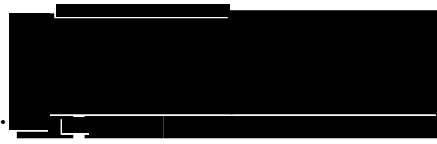

$01 / 11 / 18$

(candidate)

Date

\section{Statement 2}

I hereby give consent for my thesis, if accepted, to be available for photocopying and for inter-library loan, and for the title and summary to be made available to outside organisations.

Signed

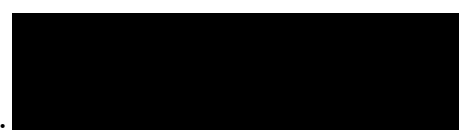

(candidate)

Date
$01 / 11 / 18$ 


\section{Acknowledgments}

Firstly, I would like to thank Dr Rajesh Ransing for his continued dedication, support and guidance and I'd like to thank Prof Mike Friswell for his advice and supervision during this time. I'd also like to thank Dr Isaac Sazonov for his help and Dr Mahmoodi Pooya for his input to get me started and work that has preceded mine.

I also want to thank Prof Cathy Holt from Cardiff University for her insight and expertise and Paul Drayton along the rest of the staff at the Artificial Limb and Appliance Centre at Morriston Hospital for welcoming me to the clinic. Their shared experience and consultation helped me understand the clinical needs of the patients and guided the direction of this research. I'd like to thank the staff at the College of Engineering at Swansea University for providing a vibrant research environment to work in and the Engineering Research Network Wales for funding my research.

Finally I'd like to give special thanks to my family for their love and support over the years and my partner, Maria, for her continued devotion and patience that has given me the encouragement to complete this work. 


\section{Contents}

Abstract $\quad 3$

$\begin{array}{lr}\text { Acknowledgements } & 6\end{array}$

1 Introduction $\quad 29$

1.1 Aims \& objectives . . . . . . . . . . . . . . . . . . . . 31

1.2 Thesis layout . . . . . . . . . . . . . . . . . . 32

1.3 International contributions from work . . . . . . . . . . . . . 33

2 Literature review 35

2.1 The biomechanics of walking . . . . . . . . . . . 36

2.1.1 Goals of walking . . . . . . . . . . . . . . . . 37

2.1.2 Gait Analysis . . . . . . . . . . . . . . . . 37

2.1.3 Muscle activation and metabolic energy costs . . . . . . . . . 41

2.1.4 Finding the COM of the human body . . . . . . . . . . . 43

2.1.5 Walking vs. Running ................ . 44 
2.1.6 Roll-over shape .................. . . 45

2.2 Predictive models for walking . . . . . . . . . . . . . 47

2.2.1 Musculoskeletal models . . . . . . . . . . . . . . . . 47

2.2.2 Inverted pendulum and compass gait . . . . . . . . . . . . 49

2.2.3 Limit cycles and stability . . . . . . . . . . . 51

2.2.4 Other extensions to the compass walking model . . . . . . 53

2.2.5 Spring loaded inverted pendulum . . . . . . . . . . 54

2.2.6 Virtual Pivot Point . . . . . . . . . . . . . . 57

2.3 Asymmetry of walking . . . . . . . . . . . . . . 58

2.3.1 Amputee gait ................. 59

2.3.2 Prosthetic leg mass imbalance . . . . . . . . . . . . . 60

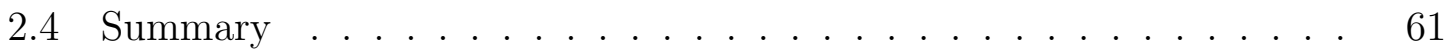

3 Asymmetric passive dynamic walker $\quad 63$

3.1 Equations of motion for the passive walker . . . . . . . . . . . . 64

3.1.1 Rolling contact ................. 65

3.1 .2 Stance phase ................. 66

3.1.3 The double support transition phase .......... . 69

3.1.4 Numerical implementation . . . . . . . . . . . . . . . . 72

3.1.5 Validation of results ............... . . 74 
3.2 Understanding results . . . . . . . . . . . . . . . . . . . . . 74

3.2 .1 Values used for parameters . . . . . . . . . . . . . 74

3.2.2 Limit cycles and a complete walking step . . . . . . . . 75

3.3 Point foot vs. curved foot $\ldots \ldots \ldots \ldots \ldots \ldots$

3.3 .1 Gait characteristics . . . . . . . . . . . . . . 79

3.4 Investigating asymmetry in the passive walker $\ldots \ldots \ldots . \ldots 81$

$3.4 .1 \quad$ Asymmetric leg masses . . . . . . . . . . . . . . . . 81

3.4 .2 Asymmetric roller radius, $R \ldots \ldots \ldots \ldots$

3.5 Discussion of results $\ldots \ldots \ldots \ldots \ldots$

$\begin{array}{lll}4 & \text { Asymmetric spring loaded inverted pendulum } & 89\end{array}$

4.1 Equations of motion for the spring loaded inverted pendulum . . . . 90

4.1.1 Double-support phase . . . . . . . . . . . . . . 93

$4.1 .2 \quad$ Numerical implementation . . . . . . . . . . . . . . . 94

4.1 .3 Validation of results . . . . . . . . . . . . 95

4.2 Evaluating model results . . . . . . . . . . . . . . . . 96

4.2 .1 Limit cycle of stable walking solution $\ldots \ldots \ldots$. . . . 96

4.2 .2 Ground reaction forces $\ldots \ldots \ldots \ldots$

4.2 .3 Walking parameter domains $\ldots \ldots \ldots \ldots$

4.2 .4 Gait characteristics . . . . . . . . . . . . . . 100 
4.2.5 Standard model parameters used . . . . . . . . . . . . 100

4.3 Investigating asymmetry in the spring-loaded inverted pendulum . . . 101

4.3.1 Asymmetric touch-down angle, $\alpha \ldots \ldots \ldots$

4.3.2 Asymmetric leg stiffnesses, $k \ldots \ldots \ldots \ldots$. . . . . . . 104

$4.3 .3 \quad$ Asymmetric roller radius, $R \ldots \ldots \ldots$

4.4 Discussion of results . . . . . . . . . . . . . . . . . 108

$\begin{array}{lll}5 & \text { Spring loaded passive walker } & 111\end{array}$

5.1 Equations of motion for spring-loaded passive walker . . . . . . . 111

5.1.1 Derivation of the equations of motion . . . . . . . . . 113

5.1.2 Double-support phase equations of motion . . . . . . . . 115

5.1.3 Collision mechanics before double-support . . . . . . . . 118

5.1 .4 Numerical implementation . . . . . . . . . . . . . . . . . 119

5.2 Limit cycle of stable walking solution . . . . . . . . . . . . . 119

5.3 Parameter domain for SLPW model . . . . . . . . . . . . . 120

5.4 Model parameters . . . . . . . . . . . . . . . . . . . . . . . 121

5.5 Investigating asymmetry in the spring-loaded passive walker $\ldots \ldots$.

$5.5 .1 \quad$ Asymmetric leg masses . . . . . . . . . . . . . . . . 123

$5.5 .2 \quad$ Asymmetric leg stiffness,$k \ldots \ldots \ldots \ldots$

$5.5 .3 \quad$ Asymmetric roller radius, $R \ldots \ldots \ldots \ldots$ 
5.6 Discussion of results . . . . . . . . . . . . . . . . . . . 131

6 Conclusions 135

6.1 Motivation . . . . . . . . . . . . . . . . 135

6.2 Discussion of Results . . . . . . . . . . . . . . . . . 136

6.3 Future work . . . . . . . . . . . . . . . . . . . . 138

A Calculating the Ground Reaction Forces $\quad 141$

$\begin{array}{ll}\text { Bibliography } & 144\end{array}$ 


\section{List of Figures}

1-1 The human leg system may be analogous to simple models such as (a) the compass model, (b) the spring-loaded inverted pendulum or (c) the proposed spring-loaded compass walking model with rolling contact on curved feet. . . . . . . . . . . . . . . . .

2-1 The gait cycle divided into different events according to Perry [107]. (Obtained from Stöckel et al. [136].) . . . . . . . . . . . . . . . . . 38

2-2 Infographic for the phases of the step during the gait cycle. Grey shading signifies stance time and white for swing time. The first $\sim 10 \%$ of a step is in double-support. . . . . . . . . . . . . . . . . . . 40

2-3 Mean and $3 \sigma$ (shaded) joint angles and torques from 120 unperturbed (red) and 519 perturbed (blue) gait cycles. Subjects walked on a treadmill and were longitudinally perturbed during stance phase with random fluctuations of the treadmill belt speed. (Obtained from Moore et al. [100].) . . . . . . . . . . . . . . . . . . .

2-4 A standard Ground Reaction Force (GRF) plot normalised by stance time and body weight. The solid line represents the vertical GRF, dotted line the fore-aft horizontal GRF. . . . . . . . . . . . . 
2-5 Energy efficiencies of walking and running at different walking speeds show that running is optimal while moving faster than $\sim 2.5 \mathrm{~m} / \mathrm{s}$. The blue line shows walking energy relationship $\dot{E}_{w}$ (Equation 2.1). The red line shows the running relationship $\dot{E}_{r}$ (Equation 2.2). These equations and associated variables are obtained from Long and Srinivasan $[85] \ldots \ldots \ldots \ldots \ldots \ldots \ldots \ldots$

2-6 Roll-over shape is obtained from motion capture data and represents an effective rocker during stance phase. (a) Markers show the trajectory of the ankle and hip, while force plates obtain the COP locations in a laboratory based coordinate system. (b) Trajectory of COP plotted in a local coordinate system aligned with the stance leg. . . . . . . .

2-7 OpenSim, University of Stanford [13]. Experimental data such as body kinematics or GRFs are input into a dynamic model which depicts physiological-like muscles and ligaments. Muscle powers and joint moments are estimated from a forward dynamic simulation. . . . . . .

2-8 Inverted pendulum model to describe the arc formed by the trajectory of the centre of mass (COM) during the single-support stance phase of walking. . . . . . . . . . . . . . . . .

2-9 A passive dynamic walking with a point mass at the hip and a point mass for each leg. Releasing it on a slope with stable initial conditions it will walk with a steady, symmetric gait with potential energy gained from the slope and energy spent mainly through collisional losses as the leg makes contact with the ground. . . . . . . . . . .

2-10 Period doubling of stable walking motions in the simple compass walker. Dotted line represent stable limit cycles. (Obtained from Garcia et al. $[37] . \ldots \ldots \ldots \ldots \ldots \ldots \ldots$ 
2-11 The inverted pendulum and spring-mass system are the standard models for walking and running respectively. The model-predicted stance dynamics (red lines) only fit experimental data (black lines) for the running model. (Obtained from Geyer et al. [39].) . . . . . . . . . .

2-12 Stick figures (left) of walking patterns with different apex conditions. The emphasised events in the left column are touch down (TD), the instant vertical leg orientation (VLO) and lift off (LO) of the stance leg. The right column shows normalised ground reaction forces of a complete gait cycle, with the black leg being the GRF of the stance leg and the overlaid grey line being the GRF of the opposite leg. (Obtained from Rummel et al. [113].) . . . . . . . . . . . . . . . . .

2-13 Schematic describing the Bipedal Trunk Spring Loaded Inverted Pendulum (BTSLIP) model. Locomotion is characterised by elastic axial leg function, leg swinging and balancing. (Obtained from Sharbafi and Seyfarth [126] and originally Lipfert [80].) . . . . . . . . . . .

3-1 A schematic of the proposed smooth rolling passive walker with unbalanced masses. . . . . . . . . . . . . . . . . . . . . 64

3-2 COP of the foot contact in the local coordinate system aligned with the leg is at $\left[x_{\theta}, y_{\theta}\right]$. The arc length is represented in the global coordinate system aligned with floor as $\left[s_{\theta}, 0\right] \ldots \ldots \ldots \ldots$

3-3 Orientation of the biped walker at double-support transition pre-impact. 69

3-4 Flowchart of a complete walking step in the PW model. Can be integrated as the 'Complete Walking Step' block in Figure 3-5. . . . . . . 
3-5 Flowchart of the algorithm used to plot bifurcation diagrams. One parameter is altered independently. If the walker does not reach a stable solution of 200 steps within 500 attempts, the initial set of parameters is seen as a 'failure' and a cycle with the next input parameter is attempted. . . . . . . . . . . . . . . . . . .

3-6 Comparison between the current PW model results (blue) and the results obtained from Goswami et al. [41] (black). Model parameters are mass ratio $\mu=2$ and length ratio $\beta=1$ (see Section 3.2.1). The parameter being altered in these bifurcation diagrams is the angle of the ground decline,$\gamma \ldots \ldots \ldots \ldots \ldots \ldots \ldots$

3-7 Schematic to show how the phase-plane of $\boldsymbol{q}$ evolves over a walking step. The blue line represents the support leg, while the red line shows the evolution of the swing leg. The diamonds represents the point at which a heel-strike condition occurs and the conditions 'jump' as the walker reaches another step. . . . . . . . . . . . . . . 76

3-8 Periodic limit cycles of the point foot model at a slope angle of 4, 5.4 and 5.7 degrees with the phase plane diagrams (right) plotted for the last 32 steps. Plots on the left show that stable gait is achieved within a few walking steps where each dot represents $\theta_{2}$ at the end of the step. Phase-plane limit cycles of the left leg (red) and right leg (blue) show that symmetric periodicity can be seen. . . . . . . . . . . . .

3-9 Bifurcation diagram of the point foot contact zoomed in. The red square shown in (a) is stretched to plot (b) and the blue box in Figures (a) and (b) is stretched to obtain plot (c). . . . . . . . . . 79

3-10 Bifurcation diagram comparisons between point foot (black) and roller foot (blue) PW models as a function of slope angle, $\gamma$. The curved foot has a radius of $30 \%$ of the leg length. . . . . . . . . . . 
3-11 Length ratio $\left(\beta_{A}=\frac{U_{A}}{L_{A}}\right)$ and mass ratio $\left(\mu_{A}=\frac{m_{H}}{m_{A}}\right)$ of leg $A$ remains the same, while $\beta_{B}$ or $\mu_{B}$ for leg $B$ varies. . . . . . . . . . .

3-12 Effect of changing the leg mass ratio $\mu_{B}$ of one leg on the gait characteristics of the PW with a slope angle of $2^{\circ}$. The blue points represents gait characteristics while the unaffected leg is the support leg, while red points indicates that the modified leg is the support. The other leg remains at $\mu_{A}=3.6 \ldots \ldots \ldots$. . . . . . . . . . .

3-13 Effect of changing the leg length ratio $\beta_{B}$ of one leg on the gait characteristics of the PW with a slope angle of $2^{\circ}$. The blue points represents gait characteristics while the unaffected leg is the support leg, while red points indicates that the modified leg is the support. The other leg remains at $\beta_{A}=0.6 \ldots \ldots \ldots$

3-14 Effect of changing the radius of curvature $R_{B}$ of one leg on the gait characteristics of the PW with a slope angle of $2^{\circ}$. The blue points represents gait characteristics while the unaffected leg is the support leg, while red points indicates that the modified leg is the support. The other leg remains at $R_{A}=30 \%$ leg length. . . . . . . . . .

4-1 Spring loaded inverted pendulum (SLIP) model with roller foot during single-support. . . . . . . . . . . . . . . . . . . . . 91

4-2 SLIP model with roller foot during double-support. The front leg's load increases, as the rear leg extends before take-off. . . . . . . . . .

4-3 Flowchart of a complete walking step in the SLIP model. Can be integrated as the 'Complete Walking Step' block in Figure 3-5. . . . . 
4-4 Comparison between the current SLIP model results and the results obtained from Whittington and Thelen [147]. Model parameters are $k=20 \mathrm{kN} / \mathrm{m}, \alpha=20^{\circ}, m=80 \mathrm{~kg}, L=1 \mathrm{~m}$ and a target walking speed of

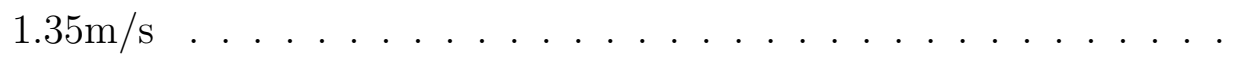

4-5 (a) Stable sequence walking up to 100 steps shows that the model converges to a steady gait from a random set of initial conditions. (b) Vertical COM trajectory for each step exhibits a repeated cyclic pattern. 96

4-6 Ground reactions forces in the X (dotted) and Y (solid) planes of the leg during stance phase (blue). The GRFs of the other leg is shown during double-support (red). . . . . . . . . . . . .

4-7 The net impulse contribution from each leg is calculated by integrating the horizontal Ground Reaction Forces (GRFs) during the total stance

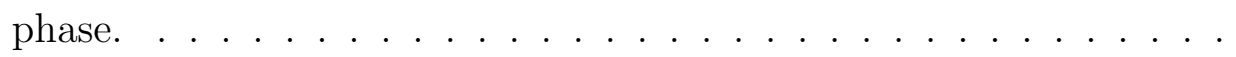

4-8 Parameter domain showing stable walking conditions at combinations of touch-down angle, effective leg stiffness and mechanical energy. Outside plots represent the vertical GRF pattern exhibiting two to six peaks seen in this parameter domain. Only two-peak vGRF patterns (region within red ellipse) are considered in this chapter. . . . . . .

4-9 Effect of changing the touch-down angle of one leg, $\alpha_{B}$, on the gait characteristics of the SLIP model. The blue points represents gait characteristics while the unaffected leg is the support leg, the red points indicates that the modified leg is the support. The other leg remains at $\alpha_{A}=20^{\circ} \ldots \ldots \ldots \ldots$ 
4-10 Vertical Ground Reaction Force (GRF) profile for the SLIP walking model with the touch-down angle of leg $B, \alpha_{B}$, being equal to (a) $15^{\circ}$ and (b) $23^{\circ}$ (shown by the red line), while the other leg touch-down angle, $\alpha_{A}$, remains at $20^{\circ}$ (shown by the blue line). The GRF plot for a symmetric model with $\alpha$ being $20^{\circ}$ for both legs is plotted as the dashed black line. . . . . . . . . . . . . . . . . .

4-11 Effect of changing the effective leg stiffness of one leg, $k_{B}$, on the gait characteristics of the SLIP model. The blue points represents gait characteristics while the unaffected leg is the support leg, the red points indicates that the modified leg is the support. The other leg remains at $k_{A}=25 \mathrm{kN} / \mathrm{m} \ldots \ldots \ldots \ldots \ldots$

4-12 Vertical Ground Reaction Force (GRF) profile for the SLIP walking model with the stiffness of leg $B, k_{B}$, being equal to (a) $17.5 \mathrm{kN} / \mathrm{m}$ and (b) $27.5 \mathrm{kN} / \mathrm{m}$ (shown by the red line), while the other leg touchdown angle, $k_{A}$, remains at $25 \mathrm{kN} / \mathrm{m}$ (shown by the blue line). The GRF plot for a symmetric model with $k$ being $25 \mathrm{kN} / \mathrm{m}$ for both legs is plotted as the dashed black line. . . . . . . . . . . . . . . 107

4-13 Effect of changing the rocker radius $R_{B}$ of one leg on the gait characteristics of the SLIP model. The blue points represents gait characteristics while the unaffected leg is the support leg, the red points indicates that the modified leg is the support. The other leg remains at $R_{A}=30 \%$ leg length. . . . . . . . . . . . . . .

4-14 Vertical Ground Reaction Force (GRF) profile for the SLIP walking model with roller radius of $\operatorname{leg} B, R_{B}$, being equal to (a) $0.1 \mathrm{~m}$ and (b) $0.4 \mathrm{~m}$ (shown by the red line), while the roller radius, $R_{A}$, remains at $0.3 \mathrm{~m}$ (shown by the blue line). The GRF plot for a symmetric model with $R$ being $0.3 \mathrm{~m}$ for both legs is plotted as the dashed black line. 
5-1 Schematic of the Spring Loaded Passive Walker (SLPW) during singlesupport stance phase. . . . . . . . . . . . . . . . .

5-2 Set-up of SLPW during double support phase. The front leg is in contact with the ground at position $\mathbf{P}_{c} \ldots \ldots \ldots \ldots$

5-3 Constrained during double-support phase with coordinates evaluated at the rear leg and Lagrangian constraints added to the front foot. . .

5-4 Flowchart of a complete walking step in the SLPW model. Can be integrated as the 'Complete Walking Step' block in Figure 3-5. . . . . 119

5-5 (a) Stable sequence walking up to 100 steps shows that the model converges from a random set of initial conditions. (b) Phase plane shows a full cycle of model for both steps. . . . . . . . . . . . . . . 120

5-6 Parameter Domain showing stable walking conditions at combinations of effective leg stiffness and mechanical energy. Outside plots represent the GRF pattern exhibiting two to six peaks seen in this parameter domain. Only two-peak GRF patterns (region within red ellipse) are considered in this chapter. . . . . . . . . . . . . . . .

5-7 Length ratio $\left(\beta_{A}=\frac{U_{A}}{L_{A}}\right)$, mass ratio $\left(\mu_{A}=\frac{m_{H}}{m_{A}}\right)$ and leg stiffness of leg $A, k_{A}$, remains the same while $\beta_{B}, \mu_{B}$ or $k_{B}$ for leg $B$ varies. . .

5-8 Effect of changing the effective leg mass ratio, $\mu_{B}$, of one leg on the gait characteristics of the SLPW model. The blue points represents gait characteristics while the unaffected leg is the support leg, the red points indicates that the modified leg is the support. The other leg remains at $\mu_{A}=3.6 \ldots \ldots \ldots \ldots \ldots \ldots \ldots \ldots$ 
5-9 Effect of changing the effective leg mass position ratio, $\beta_{B}$, of one leg on the gait characteristics of the SLPW model. The blue points represents gait characteristics while the unaffected leg is the support leg, the red points indicates that the modified leg is the support. The other leg remains at $\beta_{A}=0.6 \ldots \ldots \ldots \ldots$

5-10 Vertical ground reaction force (GRF) profile for the SLPW walking model with $\mathrm{COM}$ ratio of leg $B, \beta_{B}$, being equal to 0.67 (shown by the red line), while the other leg $\mathrm{COM}$ ratio, $\beta_{A}$, remains at 0.60 (shown by the blue line). The GRF plot for a symmetric model with $\beta$ being 0.60 for both legs is plotted as the dashed black line. . . . . . . 128

5-11 Effect of changing the effective leg stiffness of one leg, $k_{B}$, on the gait characteristics of the SLPW model. The blue points represents gait characteristics while the unaffected leg is the support leg, the red points indicates that the modified leg is the support. The other leg remains at $k_{A}=18 \mathrm{kN} / \mathrm{m} \ldots \ldots \ldots \ldots \ldots \ldots$

5-12 Vertical ground reaction force (GRF) profile for the SLIP walking model with the stiffness of leg $B, k_{B}$, being equal to (a) $16 \mathrm{kN} / \mathrm{m}$ and (b) $20 \mathrm{kN} / \mathrm{m}$ (shown by the red line), while the other leg touchdown angle, $k_{A}$, remains at $18 \mathrm{kN} / \mathrm{m}$ (shown by the blue line). The GRF plot for a symmetric model with $k$ being $18 \mathrm{kN} / \mathrm{m}$ for both legs is plotted as the dashed black line. Both maximum peaks are larger for the compliant limb. . . . . . . . . . . . . . .

5-13 Effect of changing the rocker radius $R_{B}$ of one leg on the gait characteristics of the SLPW model. The blue points represents gait characteristics while the unaffected leg is the support leg, the red points indicates that the modified leg is the support. The other leg remains

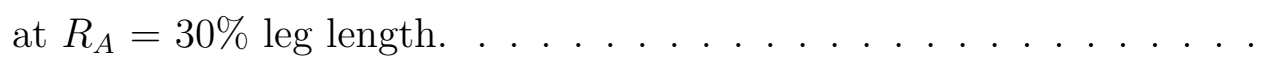


5-14 Vertical ground reaction force (GRF) profile for the SLIP walking model with the stiffness of leg $B, R_{B}$, being equal to (a) $0.26 \mathrm{~m}$ and (b) $0.33 \mathrm{~m}$ (shown by the red line), while the other leg touch-down angle, $R_{A}$, remains at $0.30 \mathrm{~m}$ (shown by the blue line). The GRF plot for a symmetric model with $R$ being $0.30 \mathrm{~m}$ for both legs is plotted as the dashed black line. . . . . . . . . . . . . . . . . . . . . 133

A-1 Calculating vertical and horizontal GRFs knowing the location of COM, COP and spring extensions. . . . . . . . . . . . . . . . . . 142 


\section{List of Tables}

3.1 Standard parameters used for the PW model. . . . . . . . . . . . . 76

4.1 Standard parameters used for the SLIP model. . . . . . . . . . . . . . 101

5.1 Standard parameters used for the SLPW model. . . . . . . . . . . . 122 


\section{List of abbreviations}

$\begin{array}{ll}\text { BOA } & \text { Basin of attraction } \\ \text { COM } & \text { Centre of mass } \\ \text { COP } & \text { Centre of pressure } \\ \text { COT } & \text { Cost of transport } \\ \text { DOF } & \text { Degree of freedom } \\ \text { GRF } & \text { Ground reaction Force } \\ \text { ME } & \text { Mechanical energy } \\ \text { PW } & \text { Passive walker } \\ \text { SLIP } & \text { Spring-loaded inverted pendulum } \\ \text { SLPW } & \text { Spring-loaded passive walker } \\ \text { vGRF } & \text { Vertical ground reaction force }\end{array}$




\title{
Nomenclature
}

\author{
$\theta_{1} \quad$ Angle of stance leg in relation to the ground \\ $\theta_{2} \quad$ Angle between the stance leg and the swing leg \\ $r_{1} \quad$ Extension on stance leg \\ $r_{2} \quad$ Extension on swing leg \\ $\boldsymbol{q}$ State vector of the system \\ $s \quad$ Arc-length of the foot from origin to point of contact \\ $\phi \quad$ Angle of the front leg in relation to the ground \\ $R \quad$ Foot roller radius \\ $\mu \quad$ Mass ratio \\ $\beta \quad$ Leg centre-of-mass ratio \\ $k_{1} \quad$ Stiffness of stance leg \\ $k_{2} \quad$ Stiffness of swing leg or front leg \\ $\gamma \quad$ Angle of the ground slope \\ $L \quad$ Original leg length \\ $y_{i} \quad$ Length from the foot to leg mass $i$ \\ $\mathbf{P}_{1} \quad$ Location of stance leg mass \\ $\mathbf{P}_{2} \quad$ Location of swing leg mass \\ $\mathbf{P}_{3} \quad$ Location of hip mass \\ $\mathbf{P}_{c} \quad$ Location of the front leg point of contact with the floor \\ $\mathbf{P}_{0} \quad$ Origin of the next walking step coordinates \\ $\boldsymbol{v}_{i} \quad$ Velocity of mass $i$ \\ $\mathcal{L}$ Lagrangian function \\ $K \quad$ Kinetic energy \\ $V \quad$ Potential energy \\ $k \quad$ Vertical constraint \\ $l \quad$ Horizontal constraint \\ $\kappa, \lambda$ Lagrangian multipliers
}




\section{Chapter 1}

\section{Introduction}

Walking is a process which people can do autonomously and requires balance and coordination between multiple limbs. The main aim for locomotion is to walk with minimum energy costs for a large period of time and with a stable, steady gait. Gait rehabilitation may involve surgery, physiotherapy, prosthetics or orthotics to give support and mimic an intact limb to walk cosmetically, reduce pain or walk more efficiently [30]. There are a number of factors that may affect gait characteristics such as pain, discomfort, neurological diseases and muscular conditions [148]; however the individual contributions are not well understood. The aim of this thesis is to investigate how the kinetic or kinematic biomechanics are affected by the purely dynamic principles of gait, assuming that human control is not a factor. This will give good insight and understanding into what may affect the passive mechanical principles of walking dynamics and will ultimately help experts make decisions in rehabilitation or the design of assistive devices.

Using dynamic models can help give valuable insight when understanding the biomechanical principles of ambulation to design better robotics, orthoses, prosthetics and aid in gait rehabilitation. Types of models used in gait research range from the simple inverted pendulum $[76,74]$ to complex musculoskeletal models [84, 135]. 


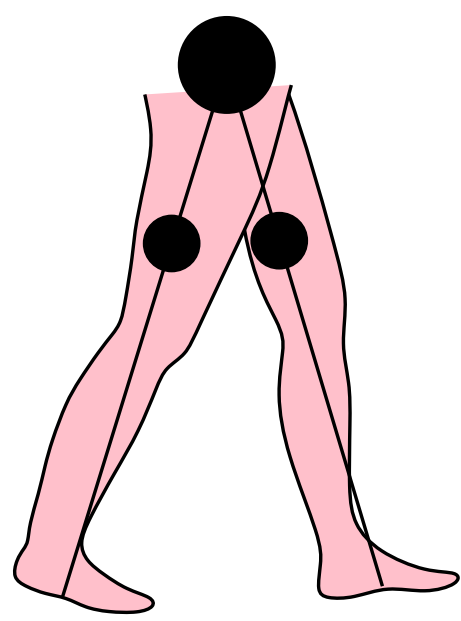

(a)

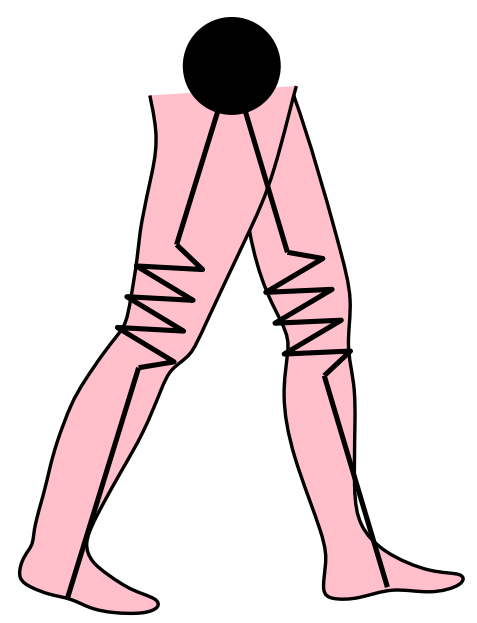

(b)

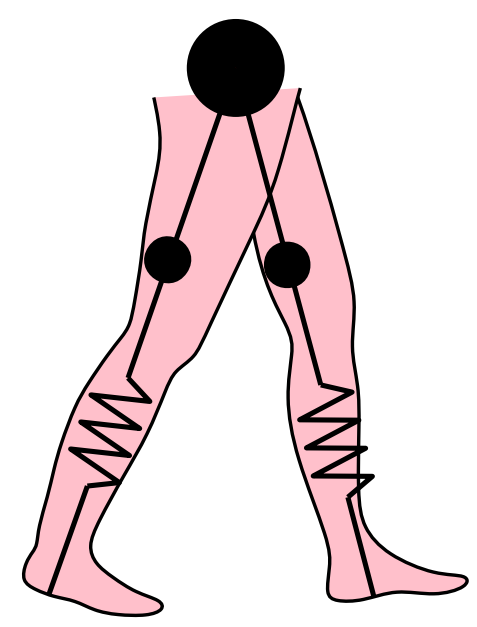

(c)

Figure 1-1: The human leg system may be analogous to simple models such as (a) the compass model, (b) the spring-loaded inverted pendulum or (c) the proposed spring-loaded compass walking model with rolling contact on curved feet.

Simple models are sometimes preferred because they reduce intricacies and have fewer unknown parameters. Stripping away the complexities allows a better understanding of the mechanics during walking without the inclusion of human neuromotor controls that alter impaired gait dynamics [102]. The inverted pendulum is commonly used to simulate the centre of mass (COM) trajectory during a walking step [124]. There has been expansive research into passive dynamic walking [94] and even the comparisons of this model to human experiments $[17,75,81]$. Recently, research has shown how a spring-loaded inverted pendulum can provide better estimations for the ground reaction forces (GRFs) [39, 81]. However, there has not been a lot of work done to investigate the asymmetry of gait using these models [55, 137]. Prosthetic leg rehabilitation makes use of the roll-over shape concept to improve an amputee's gait and symmetrise leg kinematics [18, 106], however the weight of the leg [78, 122], stiffness of the prosthesis [32, 151,33], amongst other factors [148] lead to different dynamics and hence different objectives for gait rehabilitation.

In this thesis, a passive compass walking model (Figure 1-1a) is adapted to include asymmetric masses and roller feet comparable to the human roll-over shape [47, 2]. This will be used to give an in-depth analysis of the effect of changing the inertial 
characteristics of the legs. A spring-mass walking model (Figure 1-1b) is then used to investigate asymmetric spring stiffnesses to understand human locomotion which is lacking in the passive support and propulsion provided by the muscles. As springloaded inverted pendulum models do not incorporate leg masses, a new model is derived (Figure 1-1c) which has the main purpose to examine the effect asymmetric leg inertias have on the GRFs and swing dynamics. The research in this thesis takes advantage of passive dynamic and spring-mass walking models to mimic the musculoskeletal lower limb system with reduced degrees of freedom and predict the dynamics of gait. Results are assessed by studying gait characteristics such as step length, stance time and walking speeds to inspect asymmetries and resultant outcomes between the limbs. To gain extra insight, these results are compared to human experiments to gain appreciation of the comparisons to human gait and assess the limitations of the models. Taking advantage of this insight into underlying dynamic principles will help us understand biomechanical locomotion for the use of clinical experiments and as a reference in the design of assistive devices in future such as prosthetics, orthotics or exoskeletons.

\subsection{Aims \& objectives}

The overall objective of this thesis is to use dynamic walking models without controllers to understand and quantify the mechanical effects of asymmetric gait. The types of dynamic models used are the passive walker (PW), the spring loaded inverted pendulum (SLIP) and a combined spring loaded passive walker (SLPW) model. The aims can be further broken down into a set of achievable goals:

1. Establish how well a model with no controllers predicts gait kinematics.

2. Investigate the effect asymmetric inertial loading conditions have on gait characteristics. 
3. Ascertain if the roll-over shape may be used to improve model predictions.

4. Research the effect of effective leg stiffness or altered touch-down angle as controls for gait alterations.

5. Derive the combined spring-mass/compass walking model to better predict gait dynamics.

6. Evaluate the effect of asymmetric leg weights on the gait characteristics and GRFs.

\subsection{Thesis layout}

A review of the current literature is reported in Chapter 2. It begins by establishing the defined stages of gait for a healthy individual and investigates methods of gait analysis in the laboratory. Focus is directed to introduce models that may be used to predict gait dynamics. These range from the simple inverted pendulum to complex musculoskeletal models and interest is directed towards passive dynamics and springmass models. The symmetry of healthy gait is evaluated and the effects of prostheses on gait characteristics is discussed.

A new model is proposed in Chapter 3 which is adapted from the compass walker to include asymmetric masses and rolling contact analogous to the human step (called the roll-over shape). Bifurcations of gait characteristics caused by variations of leg inertia and roller radii are investigated. A single leg is modified to see how it affects the gait asymmetries.

Chapter 4 investigates the spring loaded inverted pendulum model to match the ground reaction force pattern of human walking trials and explore the effects of asymmetric effective leg stiffnesses. The effect of an altered touch-down angle at each step is evaluated and the reasoning that this may be used as a control strategy is given. 
Chapter 5 extends this work by effectively combining both models from Chapters $3 \& 4$ to produce a new model. This representation has the purpose to improve gait predictions and investigate the effects of asymmetric leg masses on GRFs and walking characteristics. Novel formulation of conservative collision mechanics are derived and results are compared with the previous models.

Chapter 6 concludes this work to offer clinical insights and assess limitations of the models. Future recommended work includes approaches to improve/adapt the model and recommendations to incorporate this work to improve research on prosthetics or musculoskeletal models.

\subsection{International contributions from work}

- Published paper

- W. G. Charles, P. Mahmoodi, R. S. Ransing, I. Sazonov \& M. I. Friswell (2016). Comparison of point foot, collisional and smooth rolling contact models on the bifurcations and stability of bipedal walking, European Journal of Computational Mechanics, 25:3, 273-293. DOI: 10.1080/17797179.2016.1191122

- Dynamic Walking conferences

- Research poster - Optimising prosthetic feet using roll-over shape and spring stiffness to achieve symmetric walking. 4-7 June 2016. Michigan, USA.

- Presentation - A passive spring-mass model with leg masses to investigate the ballistics of swing dynamics. 4-9 June 2017. Mariehamn, Finland.

- ACME UK 23rd Conference on Computational Mechanics

- Presentation - Computational Modelling of Prosthetic Feet. 8-10 April 2015. Swansea, Wales. 


\section{Chapter 2}

\section{Literature review}

This chapter will begin by enabling us to gain an understanding of the basic biomechanical principles of healthy gait. Although the particular gait characteristics change from person to person, there is a standard definition of the normal human gait cycle. Section 2.1 outlines what information is gained from human experiments, different methods of gait analysis and why it is performed. If the norm for walking characteristics is understood, a patient's gait can be evaluated - better attention can be given to correct a person's gait.

Consideration is given to the roll-over shape - the effective rocker a person's lower limb system conforms to during the stance phase of walking. This is commonly ignored in simple pendulum models, however is shown to have remarkable consistency in human walking trials and provides a benefit to the metabolic energy cost of walking.

Section 2.2 evaluates current models that predict human walking and how insight into underlying biomechanical principles has found use in the field of robotics or general gait rehabilitation. These models range from the simple inverted pendulum model to more complex musculoskeletal anatomical models. A lot of the research into passive walking comes from the intended application of robotics that are inspired by human locomotion. These models are way of simplifying the processes of gait to 
provide a better understanding of the inertial dynamics of locomotion.

It is noted in Section 2.3 that normal walking is not completely symmetric; although in some purposes it is sufficient to assume so. Greater asymmetries in gait dynamics may be caused by a mass imbalance of the two legs from devices such as prosthetic feet, orthoses or exoskeletons. These effects will be investigated - with close attention given to the rehabilitation of healthy gait dynamics.

\subsection{The biomechanics of walking}

Bipedal walking results from a complicated process involving the brain, spinal cord, nerves, muscles, bones and joints [148]. Walking is a complex activity, which is still relatively not well understood and efficiencies are gained from a combination of evolution and self-learning. Not only that, it is difficult to define normal walking dynamics. Gait patterns vary to a great degree between some individuals [129] and it is believed that people will perform work with their muscles asymmetrically for support and progression [116]. Also, counter-intuitively, patients do not always optimise gait by minimising energy consumption - opting to expend more energy to maintain their kinematic pattern [122]. Reasons for this are unclear, however are often attributed to reducing discomfort, joint pain or to walk symmetrically for cosmetic reasons [75].

One approach to understand the biomechanics of walking is to strip down the

complexities and analyse a simple biomechanical model $[75,56]$. This is discussed in Section 2.2. This section, however, investigates the characteristics of normal walking and the methods commonly used to collect data from human experiments. Representation of the ground reaction force (GRF) pattern and methods to measure metabolic energy costs are also discussed. 


\subsubsection{Goals of walking}

When discussing locomotion from one place to the next, some simple goals for transportation need to be considered:

- Propel the body forward at a desired speed.

- Use the least amount of energy possible.

- Cause the least amount of pain for people with painful conditions.

- Robust against factors in the environment and the ability to recover after a perturbation.

Types of gaits can vary and include walking, jogging, skipping, running and sprinting. These have cyclic patterns and each limb completes a cycle in the same length of time. Walking is the most common type of gait: with the average human walking 10,000

steps a day [140]. A walking gait has a clear double-support phase during each step in which both feet are in contact with the ground (Figure 2-2). People tend to walk at preferred locomotion speeds that minimise metabolic energy spent and is comfortable to maintain for long periods of time [110].

\subsubsection{Gait Analysis}

Common pathological conditions that affect gait may be neurological; for example cerebral palsy, spastic hemiplegia, spastic diplegia, parkinsonism among other conditions. In this case, accurate diagnosis may only be possible using the techniques of systematic gait analysis [148]. Comparatively, gait abnormalities due to more peripheral disorders, such as diseases of the joints, tend to be much easier to identify. These biomechanical abnormalities may cause discomfort [28] and increase risks of further injury [114]. 
Gait analysis is a method for identifying and assessing lower limb dysfunctions in dynamic movement and loading. Patients are asked to walk in a biomechanics lab with motion capture markers placed on standard anatomical landmarks for point of reference. Biomechanical information is collected, including joint positions, joint velocities and GRF data. Skilled professionals are required for conventional gait analysis as the dynamics of walking are very complex and it can be hard to extract useful information from gait experiments. Gait analysis also requires accurate equipment and may be a long process, which will affect a patient's recovery time if it is needed for diagnosis. The force platform is used to measure the GRF and map the centre of pressure $(\mathrm{COP})$ of the foot during the gait cycle. Inverse kinematics may be used to estimate joint torques, knowing the geometry of the joints in relation to each other and GRF data.

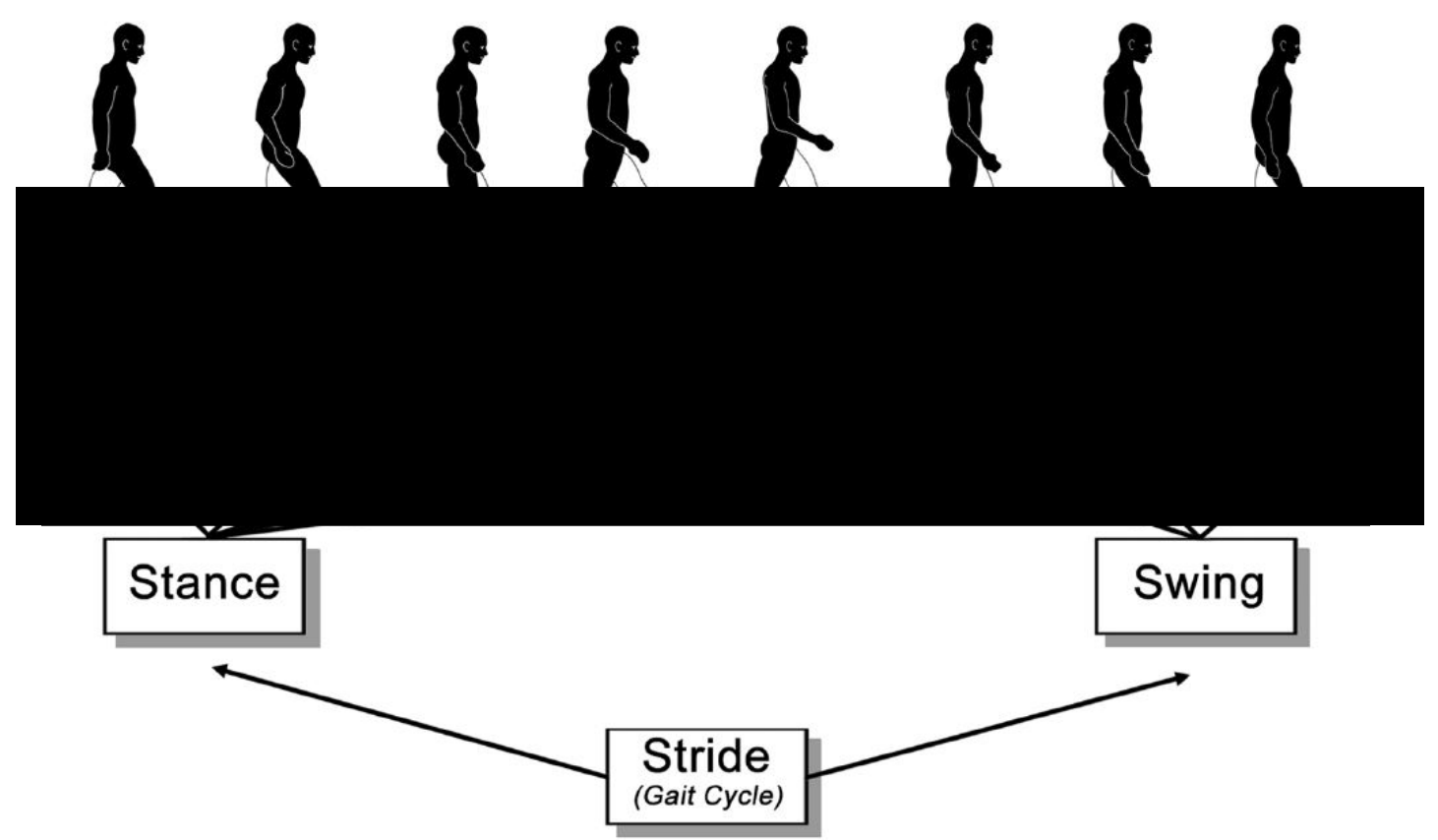

Figure 2-1: The gait cycle divided into different events according to Perry [107]. (Obtained from Stöckel et al. [136].) 
The gait cycle can be separated by different events as shown in Figure 2-1. The normal timing of these events as a percentage of the full gait cycle (heel contact to opposite heel contact) are [148, 107]:

- Stance phase

- Initial Contact - 0\%

- Loading Response - 0-10\%

- Midstance - 10-30\%

- Terminal Stance - 30-50\%

- Pre swing - 50-60\%

- Swing phase

- Initial Swing - 60-70\%

- Mid Swing - 73-87\%

- Late Swing or Terminal Swing - 87-100\%

The gait cycle is a periodic pattern involving steps and strides. Figure 2-2 shows a standard stride and the approximate percentage of each step. A step is defined as the time taken from heel-strike to opposite heel contact, a stride is the whole gait cycle until the original leg makes contact with the ground again. The double-support phase occurs when both feet are in contact with the ground and takes up approximately $10 \%$ of each step. Stance phase is defined as when the foot is on the ground and comprises of about $60 \%$ of the gait cycle. The foot that is in the air and not in contact with the floor is said to be in the swing phase of gait.

Joint kinematics is the study of the spatio-temporal positions and angular displacements of different joints in relation to each other during motion. Joint kinetics deal with the actions of forces in producing or changing the motions of the joints. In short, kinematics describe the movement (displacement, time, velocity), kinetics 


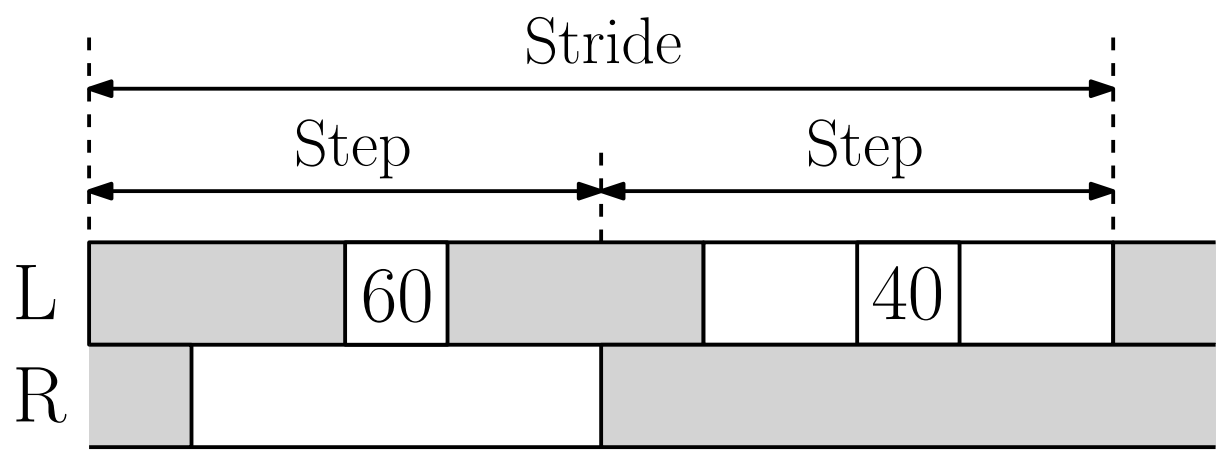

Figure 2-2: Infographic for the phases of the step during the gait cycle. Grey shading signifies stance time and white for swing time. The first $\sim 10 \%$ of a step is in doublesupport.

is the study of forces that cause motion (torque gravity, friction). In the study of gait, patterns repeat during the gait cycle and there is a typical relationship for the motion of walking at different speeds. An example of a typical kinematic and kinetic pattern is shown in Figure 2-3.

During gait analysis Ground Reaction Forces (GRFs) are measured using force plates that the patient has to step on while performing an activity to measure a response of loading. These force platforms usually measure in 3 dimensions XYZ and may be installed in treadmills [68]. The typical form of the vertical GRF during walking is an M-shaped curve with two peaks as can be seen in Figure 2-4. The second peak being the similar value to the first at self-selected speeds. At slow speeds, the peak forces are less distinct, and at higher forces, the first peak is larger, while the second peak of the vertical GRF is lower.

GRFs may help us understand the acceleration of the COM and how the body redirects it during the various stages of gait. Most people have asymmetric GRFs between each leg [51] so these results should be kept in mind for rehabilitation with patients recovering from neuromuscular or musculoskeletal injuries/diseases. 
Perturbed, $\mathrm{N}=519 \longrightarrow$ Unperturbed, $\mathrm{N}=120$
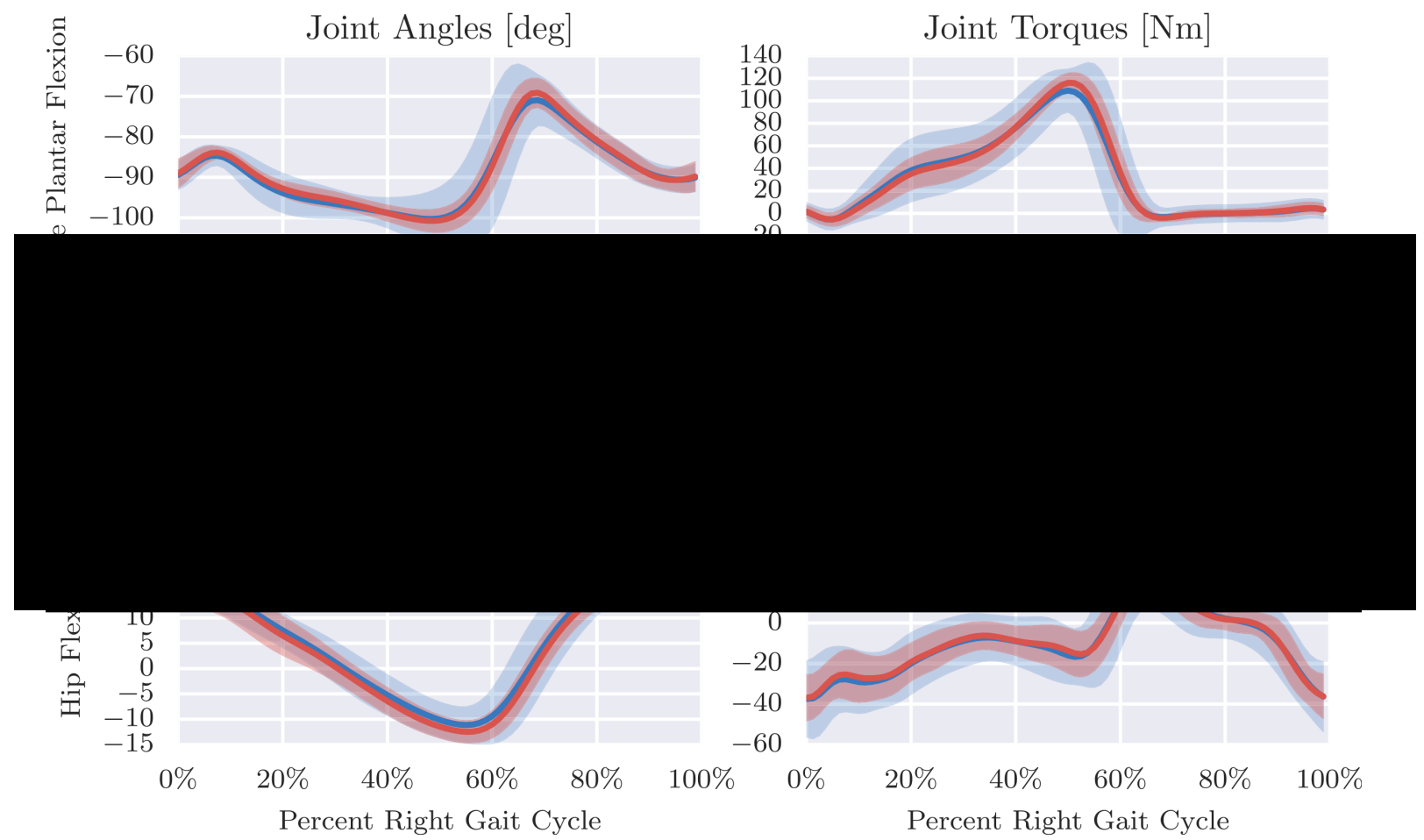

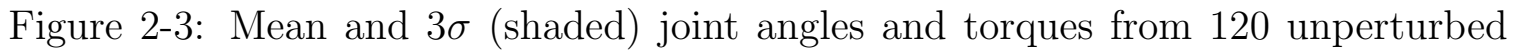
(red) and 519 perturbed (blue) gait cycles. Subjects walked on a treadmill and were longitudinally perturbed during stance phase with random fluctuations of the treadmill belt speed. (Obtained from Moore et al. [100].)

\subsubsection{Muscle activation and metabolic energy costs}

Muscles produce forces to support the body and work to lift and propel the body [115, 84]. Liu et al. [83] suggest that a relatively small group of muscles provides most of the forward progression and support needed for normal walking. Zelik et al. [153] shows that a 6 Degree of Freedom (DOF) kinematic analysis has a better energy estimate than a 3 DOF analysis for estimating biomechanical work.

Steady state walking is a cyclic action in which mechanical energy of the body remains relatively constant. An inverted pendulum model is often used to describe the energetics of gait, with the majority of work being performed during doublesupport $[11,76]$. However, net work output by muscles is highest while the COM moves upwards in early single-limb support even though kinetic and potential energy 


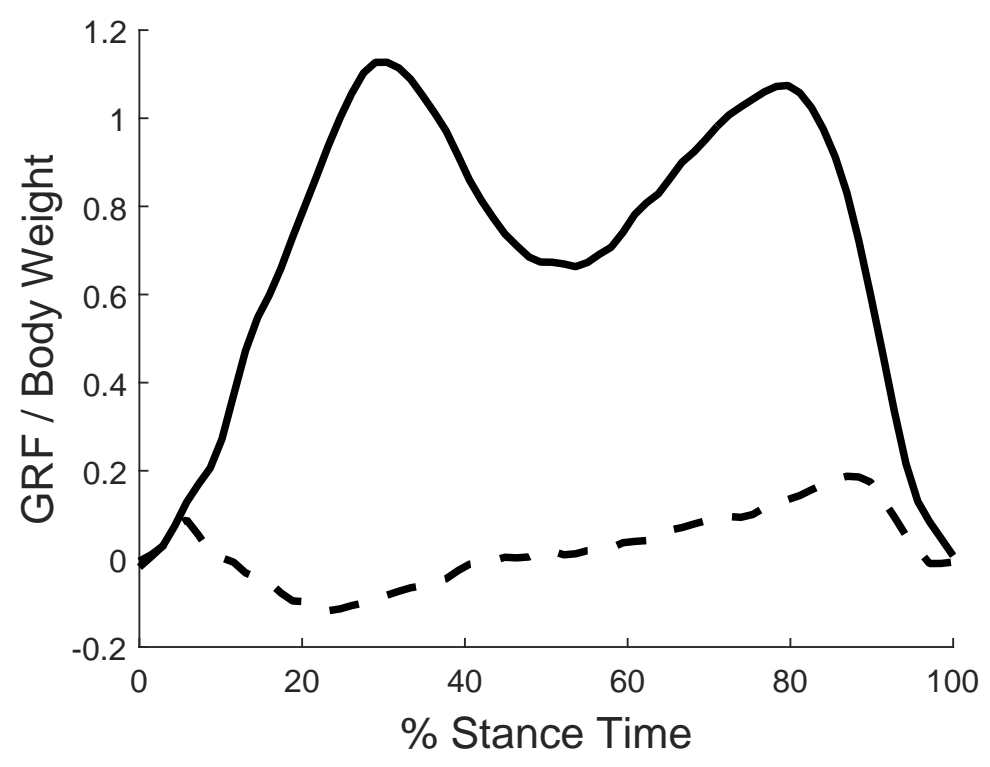

Figure 2-4: A standard Ground Reaction Force (GRF) plot normalised by stance time and body weight. The solid line represents the vertical GRF, dotted line the fore-aft horizontal GRF.

is exchanged [101]. Muscle mechanical energy cost is dominated not only by the need to redirect the COM in double support but also by the need to raise the COM in single support. High power action of the ankle extensors during late stance has been noted in research [24, 82], which causes a redirection of the body COM just before touch-down of the leading leg. This push-off before heel-strike is also shown to have a positive effect on the efficiency of bipedal robotics by decreasing the collisional loss at heel-strike [73].

One of the most important goals of walking from an evolutionary perspective, is to minimise energy spent to travel [110]. Metabolic powers can be derived from measurements of oxygen consumption and carbon dioxide production [6]. Net metabolic powers during walking are calculated by subtracting metabolic power during walking from metabolic power during standing. Energy efficiency can also be represented by the cost of transport (COT), which is expressed as energy spent per unit of distance travelled. The metabolic energy required for people to walk is mainly influenced by walking velocity and body mass, while the COT is influenced by walking velocity and 
leg length [69].

\subsubsection{Finding the COM of the human body}

From a mechanics point of view, it is important to find the COM which is a function of the positions and inertial properties of all of the limbs. During the majority of gait, the trunk is supported by one leg with the COM not above the base of support. This means that the dynamics of stance phase is analogous to an inverted pendulum

- an unstable system which requires active control or another mechanism for stability.

Three different techniques are commonly used to calculate the body's COM [38]. The sacral marker method estimates the whole body COM motion by tracking the position of the reflective marker on the sacrum of subjects as they walked. The sacrum being a large triangular bone at the base of the spine, between the two hip bones of the pelvis. The body segmental analysis technique determines the COM from a weighted average of the centres of mass for each body segment. Anthropomorphic data from standard tables are used to estimate the mass ratios and locations of COM for each body segment $[105,119]$. The third technique involved calculating the COM motion through double integration of force platform data. Gard et al. [38] compared these techniques at finding the vertical excursion of the body $\mathrm{COM}$ and found that at slow speeds all three techniques are similar, however at higher speeds the sacral marker significantly overestimated the vertical excursion of the body's COM compared to the other two methods. Rabuffetti and Baroni [109] evaluated the difference in assuming the COM is fixed onto the pelvis (approximate) and the method of finding the COM by dividing the body into 16 segments and determining their inertial properties from anthropometric studies (analytical). They determine that the COM approximate estimation is less accurate, but may be worth implementing depending on the type of experiment as it may reduce complexity.

Zatsiorsky and Seluyanov [150] use bony landmarks as reference points for locating 
the COM of body segments. de Leva [20] improves on this and provides tables that gives good inertial parameters of body segments based from joint centres that are more commonly used by researchers today. Schüler [119] provides a more recent comparison of different approaches to estimate body segment parameters.

\subsubsection{Walking vs. Running}

A running gait is defined to have a stance phase with only one limb touching the ground and an aerial phase in which no limbs are in contact with the ground [31]. At a speed of greater than $2-2.5 \mathrm{~m} / \mathrm{s}$ people tend to prefer running instead of walking. At this transition a running gait is more energy efficient; with velocity, body mass and leg length being the main influences for metabolic energy cost [69].

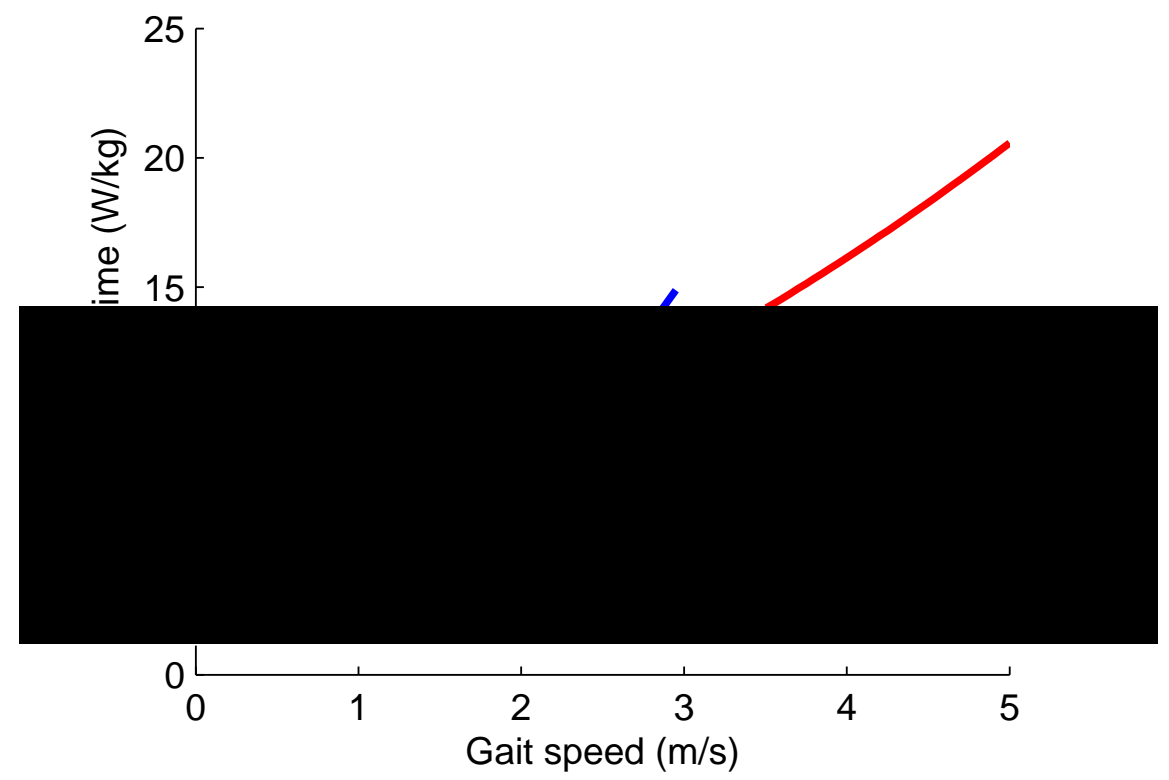

Figure 2-5: Energy efficiencies of walking and running at different walking speeds show that running is optimal while moving faster than $\sim 2.5 \mathrm{~m} / \mathrm{s}$. The blue line shows walking energy relationship $\dot{E}_{w}$ (Equation 2.1). The red line shows the running relationship $\dot{E}_{r}$ (Equation 2.2). These equations and associated variables are obtained from Long and Srinivasan [85].

Long and Srinivasan [85] used a quadratic function to fit energy expenditure for walking and running with speed and this can be seen in Figure 2-5. The walking 
metabolic rate can be expressed as

$$
\dot{E}_{w}=a_{0}+a_{2} v^{2}
$$

using $a_{0}=1.91 \mathrm{~W} \mathrm{~kg}^{-1}, a_{2}=1.49 \mathrm{~W}\left(\mathrm{~ms}^{-1}\right)^{-2}$. For running, while it is hard to distinguish between linear and quadratic models using metabolic data, the quadratic model for running is given by

$$
\dot{E}_{r}=b_{0}+b_{1} v+b_{2} v^{2}
$$

with $b_{0}=5.17 \mathrm{~W} \mathrm{~kg}^{-1}, b_{1}=1.38 \mathrm{~W}\left(\mathrm{~ms}^{-1}\right)^{-1}$ and $b_{2}=0.34 \mathrm{~W}\left(\mathrm{~ms}^{-1}\right)^{-2}$ and all values obtained from Long and Srinivasan [85].

A characteristic of walking is that the foot lands on the heel, while in a running gait the foot contact is further forward, closer to the ball of the foot. The standard model used to simulate simple walking is the inverted pendulum with locked knees, while the running model is usually a spring-loaded inverted pendulum with unlocked knees like a pogo stick.

\subsubsection{Roll-over shape}

During stance phase, a person's lower limb system conforms to an effective rocker from initial heel-strike to contralateral heel contact. The roll-over shape is found by taking measurements of the hip, knee, ankle, and centre of pressure (COP) during walking and defining a local coordinate system along the shank of the leg (Figure 2-6). This rocker or roll-over shape determines the trajectory of COM that a person conforms to during walking. Studies have found that the roll-over shape of non-disabled lowerlimb systems does not change appreciably with walking speed [47], shoe heel height [45], while carrying extra weight [46] and walking on inclined surfaces [48]. The rollover shape may be a simple tool in order to asses a person's gait and for the design and evaluation of lower limb prosthesis [18]. 


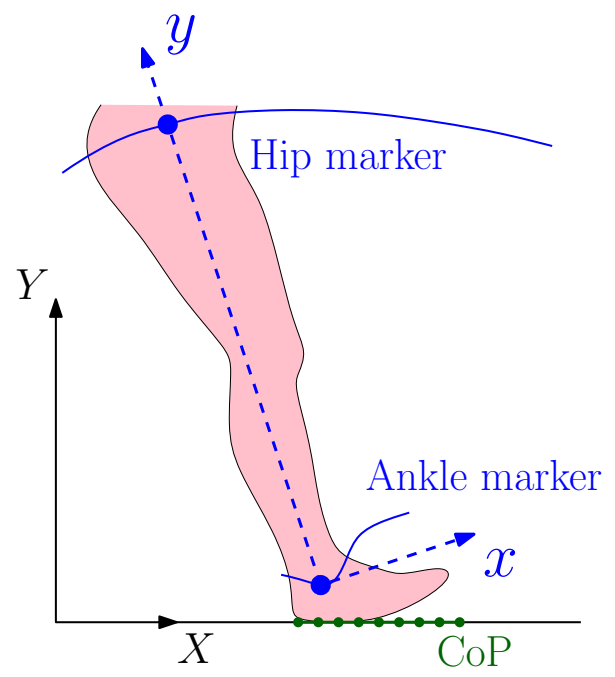

(a) Global coordinate system.

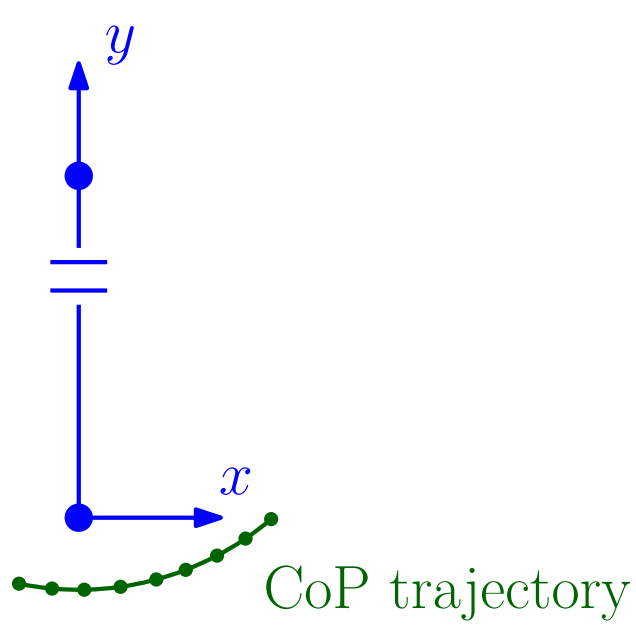

(b) Local coordinate system.

Figure 2-6: Roll-over shape is obtained from motion capture data and represents an effective rocker during stance phase. (a) Markers show the trajectory of the ankle and hip, while force plates obtain the COP locations in a laboratory based coordinate system. (b) Trajectory of COP plotted in a local coordinate system aligned with the stance leg.

Adamczyk et al. [2] found the effective rocker radius to minimise metabolic consumption is approximately $1 / 3$ of the leg length. They also found that larger arc radii resulted in smoother collisions. Wang and Hansen [145] experimented with people walking wearing rocker shoes of different radii. They confirmed that the ankle responded to changes in rocker shoe radius and walking speed to maintain a consistent ankle-foot roll-over shape. Additionally, it seems the largest changes to kinematics were at the ankle joint, rather than knee flexion, hip flexion or pelvic obliquity. This study suggests that consistent ankle-foot roll-over shapes for walking are not simply by-products of consistent ankle kinematics. The specific reason for consistent roll-over shapes is still relatively unknown, however this phenomenon may help us understand the biomechanics of walking and a constant roll-over shape may be used to simplify passive models. 


\section{$2.2 \quad$ Predictive models for walking}

Although a vast amount of information is gained from experiments on humans, it can sometimes be difficult to gain understanding of the underlying biomechanical principles involved [17]. Experiments may only tell us so much and it is not easy to define the gold standard for healthy walking. Sometimes muscles may not perform as intended due to injury or birth abnormality. Analogous computational models can help us understand the dynamics involved in human locomotion [75]. This section outlines some biomechanical models which may simulate the human system during walking. Multibody musculoskeletal simulations consist of representations of bones, muscles, ligaments and other structures. Calculations of joint moments generated by muscles can be used to explore the effects of changing musculoskeletal geometry and other model parameters. Simple models range from the inverted pendulum model and can increase in complexity by adding the swinging leg, knee or ankle joints, motor elements or compliant links between the joints.

\subsubsection{Musculoskeletal models}

Musculoskeletal models such as AnyBody [19], BoB [128], LifeMOD, SIMM [21] and OpenSim [13] (Figure 2-7) use inverse dynamics to estimate the control systems involved in human gait. By taking a skeleton and muscle system with realistic human properties and applying the same GRFs and kinematics from experimental motion data, these models use a controller to estimate the joint torque and muscle power required to perform the same motions. However, it is not easy to predict controller changes with external perturbations or injuries without running another individual's experiment $[86,129]$.

These multibody simulations have clinical implications in decision-making pro-

cesses and have capabilities in industry including simulating movements such as walking, running, jumping, cycling and weight lifting among others. In the field 


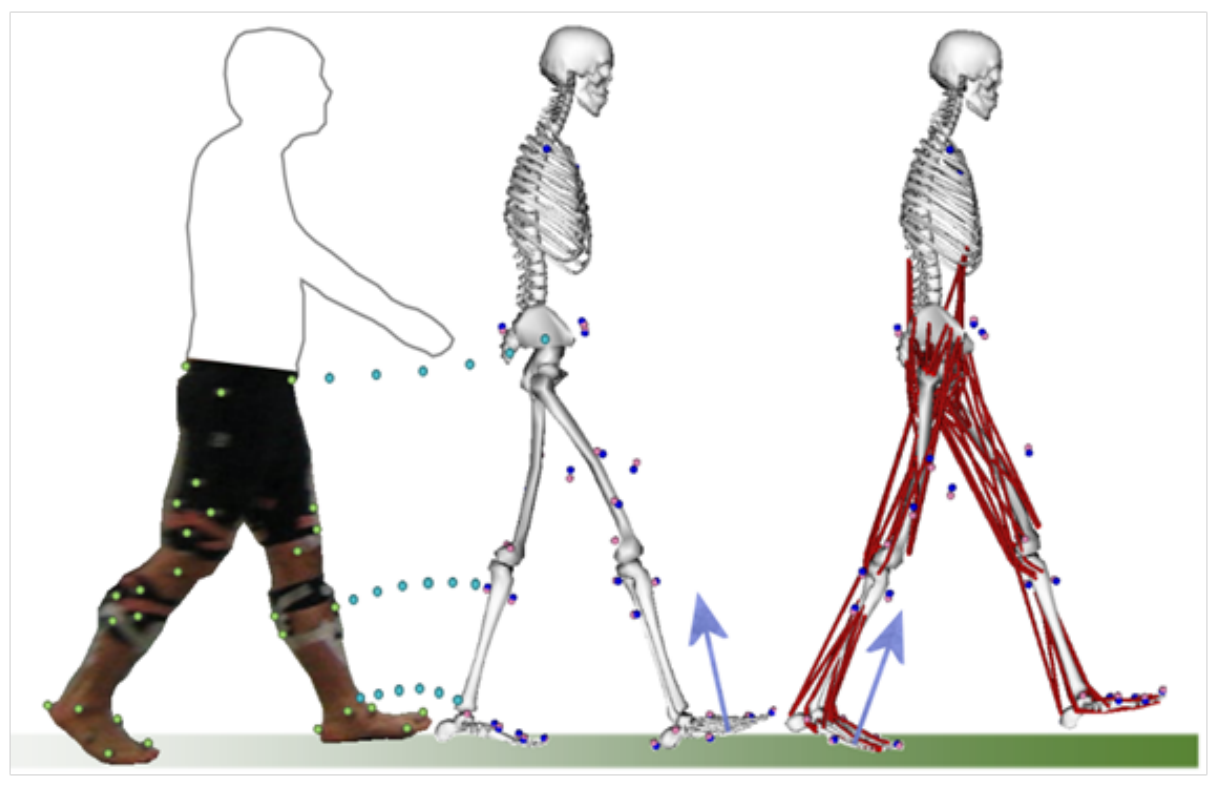

Figure 2-7: OpenSim, University of Stanford [13]. Experimental data such as body kinematics or GRFs are input into a dynamic model which depicts physiologicallike muscles and ligaments. Muscle powers and joint moments are estimated from a forward dynamic simulation.

of rehabilitation, these models have been used to understand crouch-gait [135].

The properties of these musculoskeletal models are usually based on cadaver data and scaled to fit an individual patient based on simple linear scaling laws [29]. This means that, although some good insight may be gained of the biomechanical principles during movement tasks, a model will not accurately represent an individual patient. There is also a lack of consistency among models and output differences are not simply an artefact of naturally occurring differences between individuals [144]. That work can be useful for understanding contributions from individual muscles and for gait rehabilitation [138]. Although this insight is useful for ergonomics, orthopaedics and occupational health; variations can exist due to uncertainty in marker position [87] or over-fitting of the data. 


\subsubsection{Inverted pendulum and compass gait}

The six determinants of gait was among the early concepts for understanding energy usage during walking. The hypothesis behind this theory was that in order to conserve as much energy as possible; the human system would try and minimise the amount centre of mass (COM) excursion during gait. As the body performs work in order to raise the position of the $\mathrm{COM}$, it seems intuitive that keeping the COM level during walking would minimise energy costs. It would later be discovered that humans do not maintain a constant COM height and do, in fact, follow a cyclic trajectory with the COM being raised and lowered during each step.

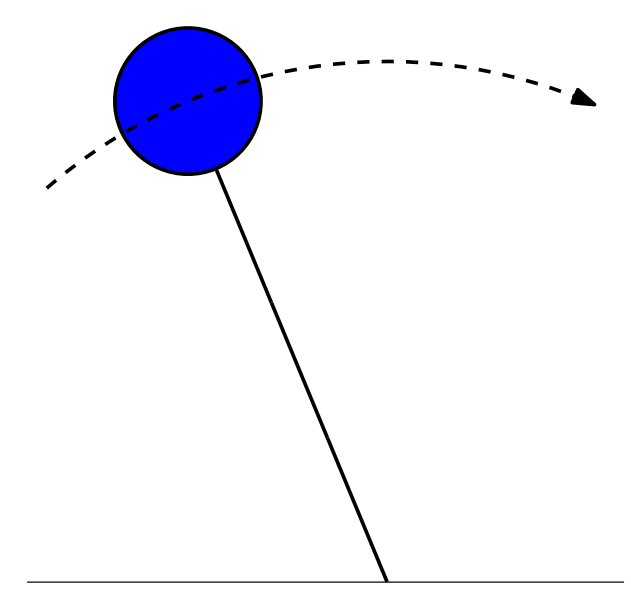

Figure 2-8: Inverted pendulum model to describe the arc formed by the trajectory of the centre of mass $(\mathrm{COM})$ during the single-support stance phase of walking.

The stance phase of a gait can be envisioned by the inverted pendulum (Figure 2-8), with the COM at the hip, the leg being a rigid link and the foot pivoting on the floor $[9,10,99]$. The body behaves like an inverted pendulum rotating about the stance foot, while the swing leg behaves like a regular pendulum about the hip. An inverted pendulum theory provides a good model for walking mechanics; the mechanical energy remains consistent, with work being performed during doublesupport phase to redirect the trajectory of the COM between each stance phase.

McGeer [94] discovered the passive dynamics of walking by building a walking mechanism that could traverse down a slope with no controllers or actuators. A 
schematic of this can be seen in Figure 2-9. This mechanism propels itself forward due to gravitational and inertial effects, exhibiting a gait comparable to that of a human. To negate foot scuffing during leg swing, raised platforms were used in experiments. Walking on a slope has the effect of supplying potential energy as energy is lost, with energy losses mainly resulting from collisions as the foot impacts on the ground. A steady symmetric gait is maintained when collisional energy is lost at the same rate potential energy is gained.
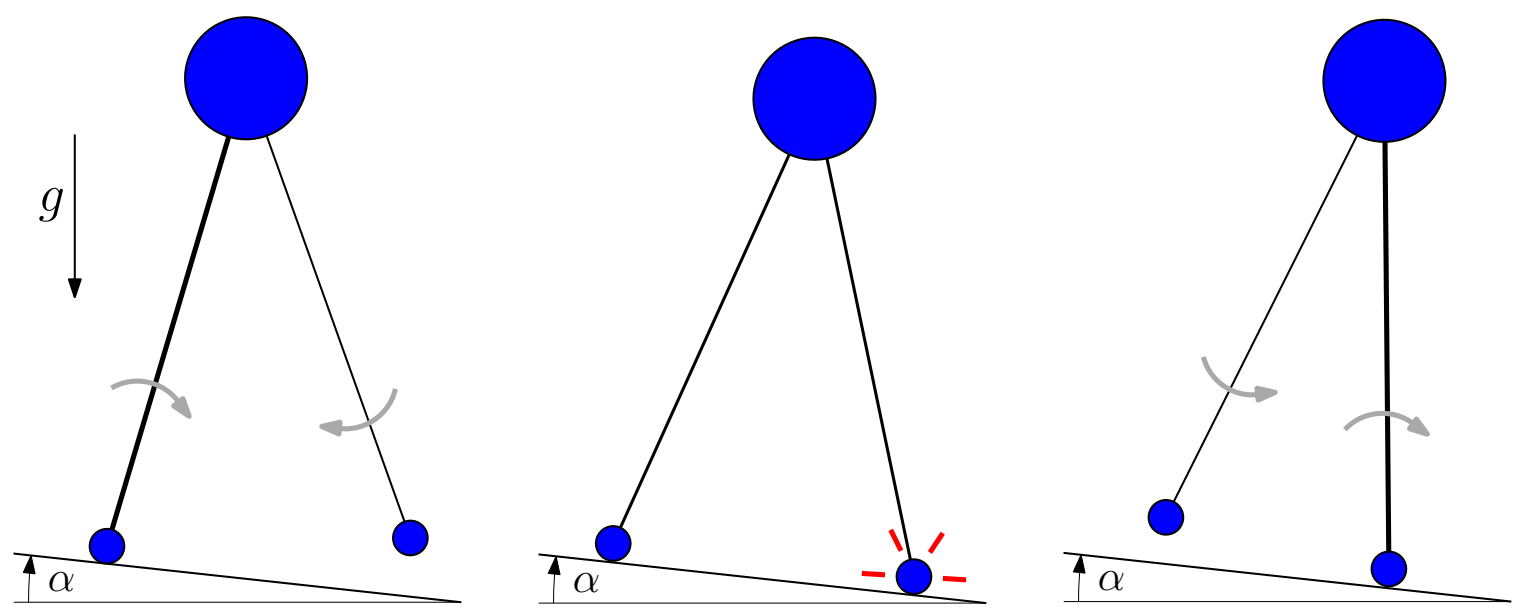

Figure 2-9: A passive dynamic walking with a point mass at the hip and a point mass for each leg. Releasing it on a slope with stable initial conditions it will walk with a steady, symmetric gait with potential energy gained from the slope and energy spent mainly through collisional losses as the leg makes contact with the ground.

The step-to-step transition is an unavoidable energetic consequence of the inverted pendulum gait [74]. As the swing leg makes contact with the ground, it is assumed that an instantaneous and inelastic collision occurs and the trailing leg loses contact with the ground. The double-support phase time is considered negligible in computations for the biped's dynamics. Although passive dynamics minimises mechanical energy losses during the stance phase; energy is used by the biped to redirect the COM during the instantaneous double-support phase.

Kuo [73] applied a torque to the stance leg and an impulse applied immediately before heel-strike to explore powered walking. It was discovered that pre-emptive push-off can reduce the COM redirection vector of the collision by as much as a factor 
of two, which results in a factor of four reduction in the energy dissipated during the collision. Kuo et al. [76] suggests that a substantial metabolic cost is associated with walking like an inverted pendulum. The work performed on the COM in step-to-step transitions can be estimated experimentally [24]. Adamczyk and Kuo [1] examined the pendulum model's predictions for how the COM is redirected between arcs and although the model predicts trends in COM velocity and work, the non-rigid human leg performs the step-to-step transition over a considerable fraction of the gait cycle (approximately 20-27\% of a stride). Also, while the inverted pendulum with a rigid leg support has been commonly used to describe gait, this model does not match the correct ground reaction forces due to the assumption that the leg is rigid [39]. An instantaneous, inelastic collision is not necessarily a good assumption for human gait. Spring-Loaded Inverted Pendulum (SLIP) models have a defined double support phase and are discussed more in Section 2.2.5.

Inverted pendulum models have been shown to predict many aspects of human walking mechanics including walking speed frequency [5], walk-run transition speeds [66], preferred walking speed - step length ratios [72], the influence of preferred step width [22], stability [37] and the relation between work-rate and stride frequency [97]. While passive dynamic walking models are inspired by human locomotion, these models may also be used to understand human gait mechanics. Collins [17] focuses on the function of arm swinging, balance and fall risk among the elderly and improvement in prosthetic foot design. Kuo and Donelan [75] suggests that these dynamic models should not be considered a solution to rehabilitation on its own, but as a useful starting point for experimental enquiry.

\subsubsection{Limit cycles and stability}

Even though the dynamics of the passive walker during the stance phase is a chaotic double-pendulum system, the walker settles onto a periodic cycle at each step [40]. As the slope angle increases (or variation of another parameter), the walker settles to 
a 2-period bifurcation and walking dynamics become asymmetric, as shown in Figure 2-10. This may increase up to n-period gate until chaotic walking and then the biped becomes unstable.

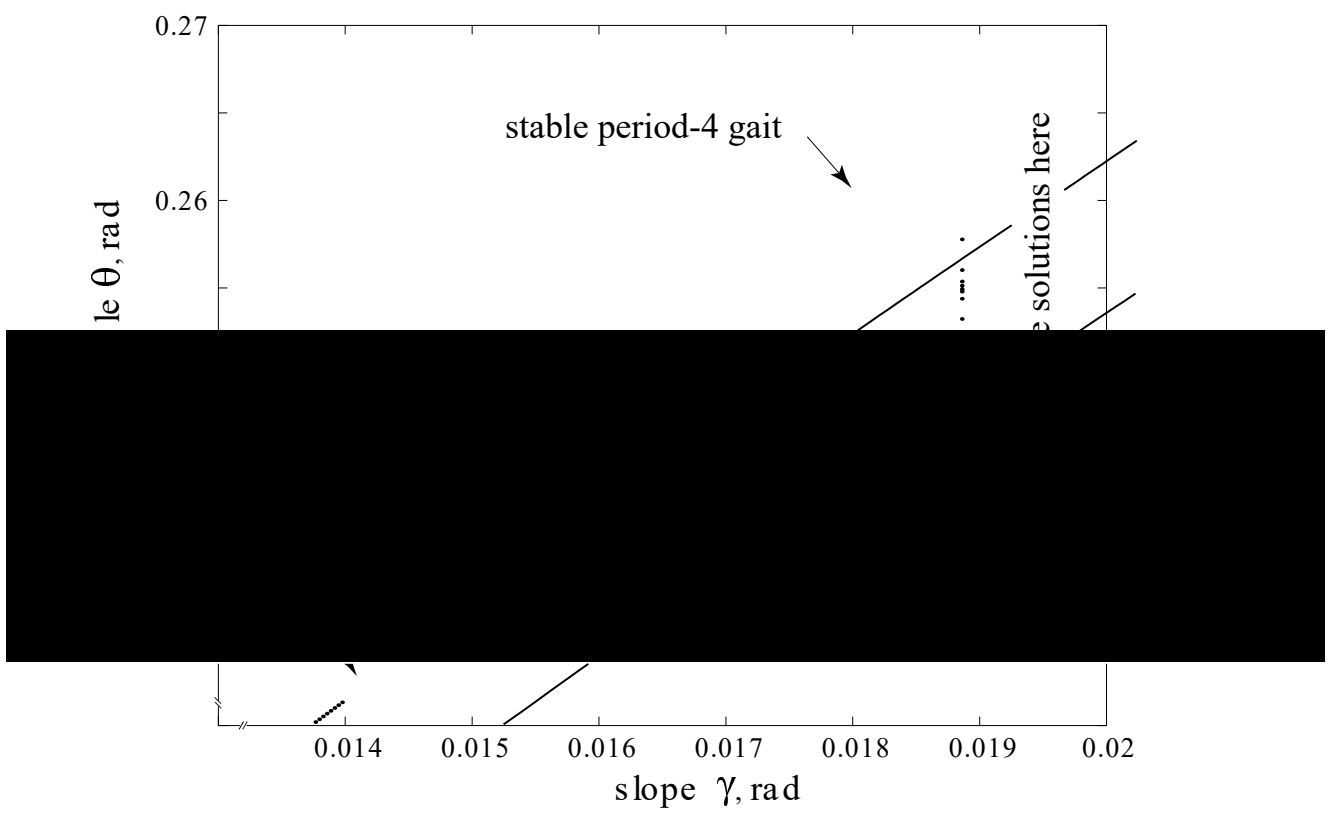

Figure 2-10: Period doubling of stable walking motions in the simple compass walker. Dotted line represent stable limit cycles. (Obtained from Garcia et al. [37].)

An active area of research is to linearise the system [37, 154]. By linearising the equations of motion, analytical methods can be used to quantify the level of stability and robustness, which equates to the ability to keep walking under random perturbations. The motivation for understanding this stability was often in the field of robotics. If the robot encounters unexpected knocks in the real world, such as surface friction, strong winds, tripping up on objects or walking on uneven terrain it will be beneficial to be able to maintain a steady walking condition without falling over. The most robust walking parameters are not necessarily found from the best linearised stability, as it is not comparable to the basin of attraction (BOA) stability [120]. If the initial conditions of the walker lie within the BOA, the model reaches a stable limit cycle without falling over. Bruijn et al. [7] notes that falling poses a major threat to the steadily growing population of the elderly and provides a review of different stability measures. Existing stability measures include zero moment point, 
Floquent multipliers and basin of attraction and disturbance rejection criteria [52].

\subsubsection{Other extensions to the compass walking model}

As discussed in Section 2.1.6, the human neuro-musculoskeletal system conforms to rolling contact while walking. Most passive dynamic walkers use point feet as a simplification to the walking dynamics, however the shape of the foot has been shown to have a direct effect on the gait behaviours [89]. A passive biped may have flat feet $[63,146]$ or arc feet $[58]$ and comparisons between them have been published $[77,61]$, showing that curved feet can increase the stable walking range and basin of attraction with flat-feet also providing an advantage over point foot by less collisional losses. Wisse et al. [149] investigated how flat feet with ankle springs can mimic arc-shaped feet. They discovered that spring stiffness has a similar effect to the foot radius and similarly it reduces sensitivity to perturbation and hence improves its disturbance handling.

Robots based on passive dynamics use small active power sources to walk on level ground [15]. Extensive work has been done to increase the complexity of the simple passive walker in other ways: Adding a torso to the upper body with the effect of increasing stability [14, 82]; Extending the model from 2D to 3D to add the frontal plane [42, 49]; Adding more passive elements to the walking model such as dampers and knees [16, 139]; Adding a damper to the hip to increase robustness and stability [41] etc. Some robots based on passive dynamics use knees to negate foot scuffing and have a more human-like looking gait. Song et al. [134] found that a spring for the swing leg - rather than a rigid pendulum - gives a better estimation of the trajectory and kinetics of the leg. Work has do be done by the walker to make up for collisional energy losses and maintain a steady gait. An impulse at the foot demonstrates how elastic ankle work can improve the efficiency of gait [152]. If the impulse is activated shortly before contralateral heel-strike, it can potentially reduce the collisional energy losses [73]. 


\subsubsection{Spring loaded inverted pendulum}

The inverted pendulum model for the stance phase of human gait assumes that the support leg is rigid. This is not the case, as the human leg is compliant during the gait cycle [1]. Experiments have shown that the tendon of the gastrocnemius medialis muscle absorbs and releases energy elastically during walking [35]. From this it can be deduced that walking efficiency does not just depend on how close the COM trajectory follows an inverted pendulum arc, but also how the lower leg musculoskeletal system performs contractile forces to support and displace the body. Although the inverted pendulum model assumes that the majority of work is performed during the doublesupport to redirect the trajectory of COM velocity [23, 73]; Neptune et al. [101]'s work suggests that a large amount of metabolic energy is used to raise the COM during early single-stance. This suggests the inverted pendulum model may not be the best approximation as it assumes a rigid support and minimal work done during the stance phase.

Spring loaded inverted pendulum (SLIP) models are usually used to model running gaits and correctly match the ground reaction forces. In research, running analysis is typically done on a SLIP, while walking is done on a rigid inverted pendulum. Figure 2-11 illustrates how the inverted pendulum model with a rigid link does not correctly match experimental GRFs, however a spring-mass inverted pendulum model is commonly used to simulate running dynamics. Note that the horizontal GRF, $F_{x}$, for the running spring-mass system in Figure 2-11 does not comprehensively match the 'wiggle' at the start of the step. This 'wiggle' in the experimental data may be due to effects not simulated in the spring-mass model such as vibration of the force plate or effects of the heel making contact with the ground before the ball of the foot $[67,65,141]$.

Geyer et al. [39] used a simple bipedal spring-mass model to show that compliant legs are essential to obtain the basic mechanics and GRF behaviour of human walking. This model found stable walking with two equal peaks for the vertical GRF that 


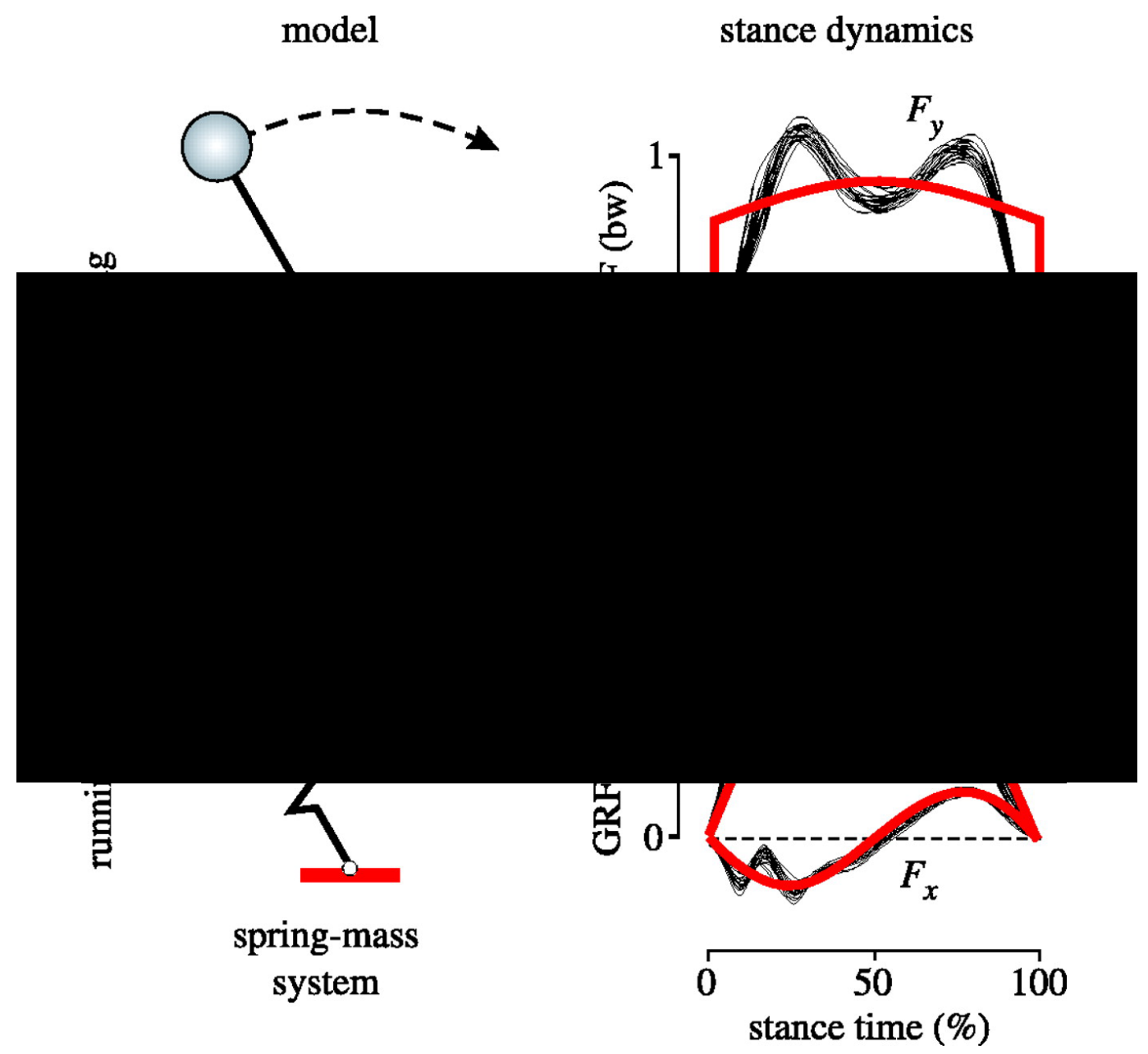

Figure 2-11: The inverted pendulum and spring-mass system are the standard models for walking and running respectively. The model-predicted stance dynamics (red lines) only fit experimental data (black lines) for the running model. (Obtained from Geyer et al. [39].)

defines the known M-shape. This suggests that the leg behaves pseudo-elastically during the stance and double-support phases.

Whittington and Thelen [147] investigated the GRFs of a simple mass-spring model with roller feet and observed a similarity with human walking. They evaluated the effect of roller radius, impact angle and leg spring stiffness and compared with human COP excursions. In this model, both the limb stiffness and limb impact angle needed to be adapted to match speed-related changes in GRFs. Both Geyer et al. [39] and Whittington and Thelen [147] assume that the leg spring velocity is zero when the leg is vertical - leading to a minimum in the GRF plot at mid-stance. However 
this is not always the case as proven in Figure 2-12 taken from Rummel et al. [113], where the vertical velocity at mid-stance may be adapted with the touch-down angle of the swing leg.

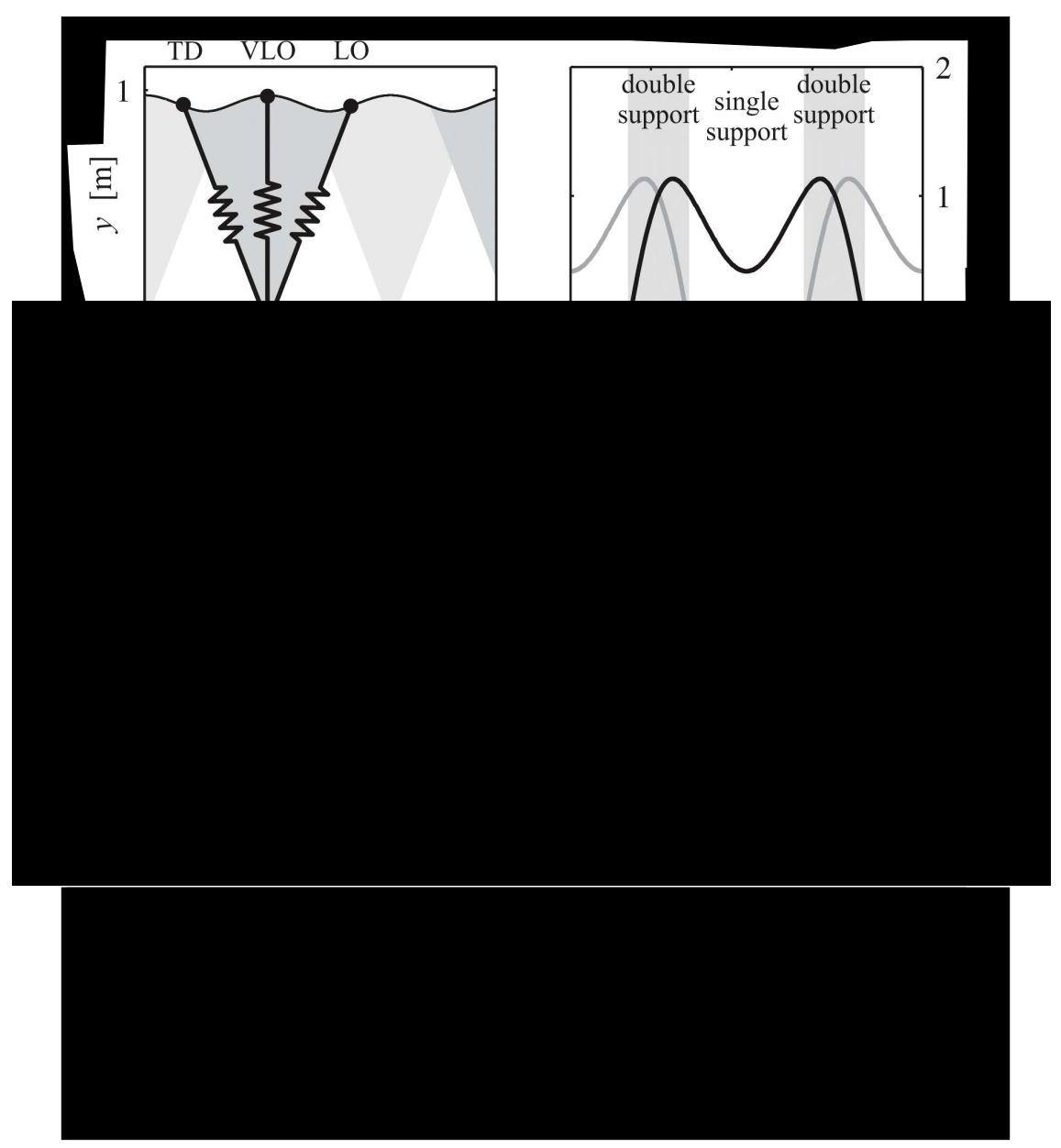

Figure 2-12: Stick figures (left) of walking patterns with different apex conditions. The emphasised events in the left column are touch down (TD), the instant vertical leg orientation (VLO) and lift off (LO) of the stance leg. The right column shows normalised ground reaction forces of a complete gait cycle, with the black leg being the GRF of the stance leg and the overlaid grey line being the GRF of the opposite leg. (Obtained from Rummel et al. [113].)

By using experimental gait data, comparisons can be made between the springmass model and the human system. Using Hooke's law for a spring, $F=k x$, taking $x$ is the distance between COM and COP (effective spring length) and $F$ from the GRFs, the effective original rest length and spring stiffness, $k$, can be found. Lipfert et al. [81] used experimental data to fit the spring-mass model for walking and running. 
The model parameters such as weight, leg rest length and effective leg stiffness were obtained through measurements and experimentation. Through this analysis they found that the spring-mass model gave accurate results at typical walking speeds $(1.04 \mathrm{~m} / \mathrm{s}$ and $1.55 \mathrm{~m} / \mathrm{s})$, but linear-spring like behaviour can not be assumed for all walking speeds. At very slow and very fast walking conditions, the force-length curve for the effective leg length exhibited a non-linear relationship.

\subsubsection{Virtual Pivot Point}

Animals and humans have been shown to point the GRF vectors above the COM towards a virtual pivot point (VPP) [93]. This creates a stable system, conceptualised with the analogy of a rocking boat; the centre of buoyancy counters the tipping forces due to gravity. The VPP approach does not use postural feedback to explain dynamic stability. This is a concept which may help us discover insight into the stability of gait; by taking unstable dynamics (an inverted pendulum) and converting it into an analogous stable system (regular pendulum). In the case of an uncontrolled inverted pendulum model, the GRF vectors point directly towards the COM and the angular momentum is conserved. Similar to the spring-mass model, the VPP concept describes how mechanical behaviour rather than a control algorithm can produce stability. Although VPP is a single position, the forces do not need to be directed towards this single point, rather generally point above the COM to produce a torque opposite of the centre of gravity moments. Sharbafi and Seyfarth [126] model the

human locomotor system as three basic sub-functions: Support from an elastic leg, leg swing dynamics and balancing. They use the bipedal trunk spring loaded inverted pendulum (BTSLIP) model as seen in Figure 2-13. 


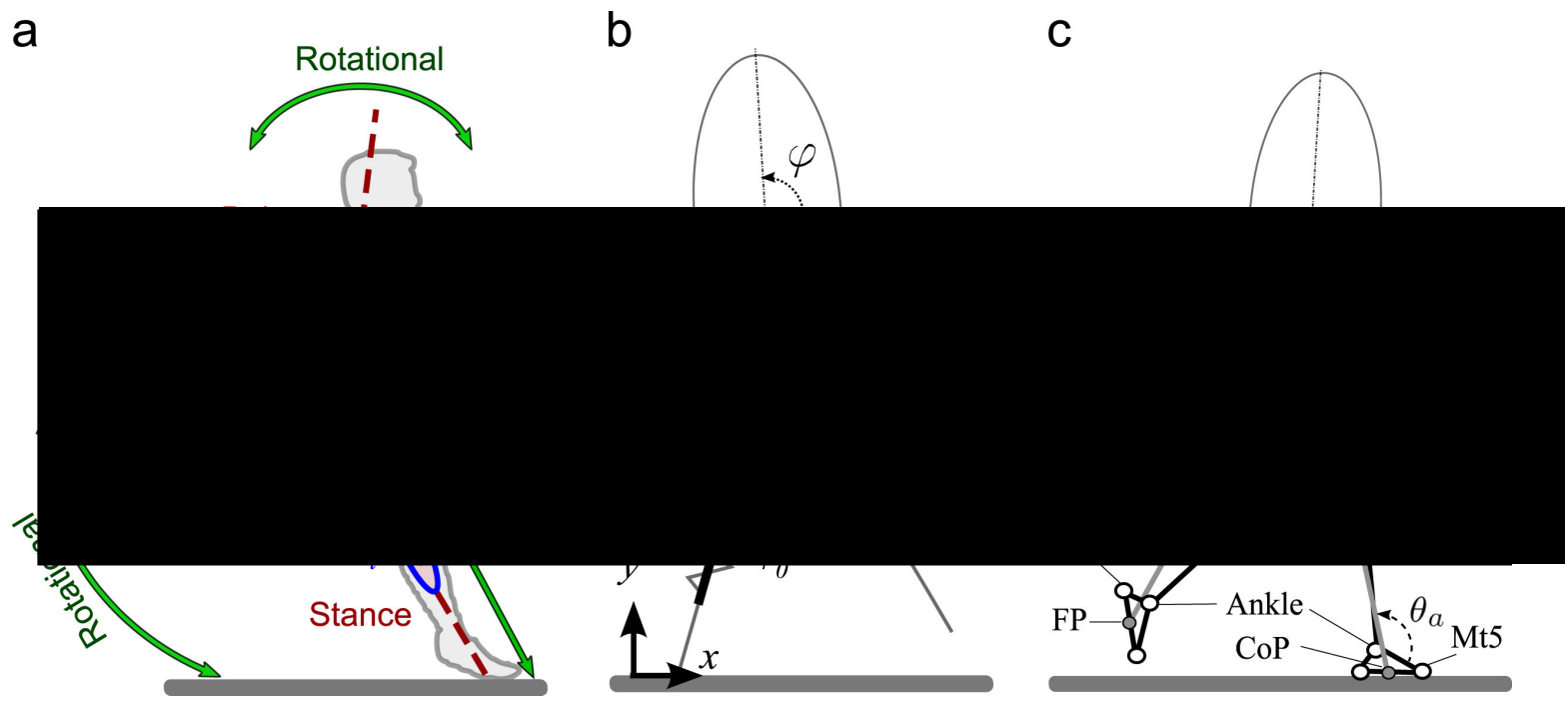

Figure 2-13: Schematic describing the Bipedal Trunk Spring Loaded Inverted Pendulum (BTSLIP) model. Locomotion is characterised by elastic axial leg function, leg swinging and balancing. (Obtained from Sharbafi and Seyfarth [126] and originally Lipfert [80].)

\subsection{Asymmetry of walking}

Asymmetric walking mechanics may be a product of stroke, injury, diseases of the joints, neuromuscular disorders etc. Persons walking with prosthetic feet have a shorter stance time on the prosthetic and higher peak forces on the contralateral intact limb. For amputees the aim of rehabilitation should not always be to symmetrise gait, as the temporal asymmetry can be considered a sensible adaptation to the impairment due to the prosthesis [53].

Some researchers suggest that healthy walking is not symmetric; one leg is more responsible for propulsion, while the other is responsible for support [130]. Sadeghi et al. [116] perform a review of symmetry and limb dominance in able-bodied gait. They suggest that although symmetry is often assumed to simplify data collection and analysis; able-bodied gait is naturally asymmetrical and may be associated with the different contributions of the lower limbs in carrying out propulsion and control. One limb is mainly responsible for supporting the body, while the contralateral limb contributes more to propulsion [115]. Herzog et al. [51] suggests that normal walking 
is asymmetric and this should be kept in mind when trying to achieve gait symmetry for patients recovering from neuromuscular or musculoskeletal accidents/diseases. However, Ankaral et al. [3] believe that although dynamical asymmetry in walking is significant and statistically distinguishable, ignoring these asymmetries in walking may be advantageous; providing not only modelling convenience, but also a more consistent and predictive model. Passive walking models also predict asymmetric walking due to 2-period bifurcations at faster speeds [43, 44], a behaviour described in Figure 2-10.

\subsubsection{Amputee gait}

Amputees are highly susceptible to fall related injury while ambulating [142] and most amputee patients are over the age of 55 [96]. Amputees walk with an asymmetrical gait, which may lead to future musculoskeletal degenerative changes $[8,71,28,114]$. Both stability and adaptability of coordination are reduced in people walking with prosthetic feet. Amputees may have an increases risk of falling because their walking pattern is less stable across a wide range of velocities and less adaptive to variations in walking velocity. The stance time of the prosthetic leg is significantly shorter than that of the normal leg $[4,59,91,25]$. The reasons for the different stance times are unclear but are usually attributed to pain or discomfort in the residual limb, instability within the socket, lack of feedback regarding foot position, strength decrements on the prosthetic side, and/or reduced confidence in supporting body weight on the prosthetic leg. [92]

Schaarschmidt et al. [118] notes that deficits of a prosthetic leg such as missing active knee extension and ankle push-off are compensated by the intact leg. Altered touch-down angle for the prosthetic enables forward propulsion while load bearing is largely shifted to the intact leg. Snyder et al. [133] tested the effect of five different prosthetic feet on the gait and loading of the sound limb. Their results (in agreement with others $[108,27,118])$ indicate that the sound limb is susceptible to increased 
vertical forces during loading, despite a reduced walking velocity; with the exception of the Flex-Foot, which they note has a flexible long keel and large arc of dorsiflexion motion. Hurley et al. [57] supports that amputees have a more asymmetrical walking pattern than non-amputees, but claim that the forces acting across the joints of the contralateral limb are not significantly higher than that of a non-amputee. Nolan et al. [103] is in agreement that transtibial amputees experienced a greater GRF peak on their intact limb compared to their prosthetic, and while this was also observed for transfemoral amputees, the difference was not significant.

\subsubsection{Prosthetic leg mass imbalance}

Designers of prosthetic feet are more inclined to use lightweight materials, being about 30-40\% times the weight of an effective physiological foot [92]. Using lightweight prostheses has previously been suggested as a contributing factor to walking asymmetries, however when the prosthetic leg is loaded as the same as the intact leg, the energy cost of walking increases [132]. Distally positioned prosthesis masses result in higher metabolic costs of walking, whereas proximally positioned prosthesis masses had less effect on metabolic costs of walking [36, 78, 123]. Mattes et al. [92] also found that matching the moments of inertia for the prosthetic and intact lower legs resulted in greater asymmetry and higher energy cost. These results support the hypothesis that matching the inertial characteristics of the legs do not result in a more symmetrical gait. Selles et al. [122] sought out to find if a perturbation of a different weight of a prosthetic foot would influence the kinematics (joint angles) or the kinetics (joint torques) of the joints during the walking cycle. This study suggests that transtibial amputees predominantly adjust to mass perturbation with a kinematic invariance strategy in which joint kinematics (walking speed, stride length and stride frequency) remain the same after mass perturbation, whereas joint torques changes. In other words - an amputee will expend more energy in order to maintain the same joint angles during walking gait. It is unknown why this strategy is employed as these 
results suggest minimisation of energy expenditure is not a driving mechanism. One reason may be that the amputee would want to walk cosmetically similar to nonamputees or match the prosthetic leg kinematics to the contralateral leg. Smith and Martin [131] recommends at least 5 minutes of walking for individuals to become well accommodated to asymmetrical changes in lower extremity inertial properties.

Mahmoodi et al. [90] used a passive bipedal walker model to investigate gait characteristics of asymmetric masses. Honeycutt et al. [55] showed that stable asymmetric walking patterns exist for PDW models by changing mass, mass location, knee location and leg length of one leg. Rasouli et al. [111] extended previous passive dynamic models by making the legs asymmetric. They compared the models' unstable boundaries, stable periodic intervals and average velocity of motion. They demonstrated that asymmetric passive walkers are more sensitive to changes of mass ratio.

\subsection{Summary}

This literature review evaluates how clinicians and researchers assess a person's gait for the use of gait rehabilitation and how biomechanical models are used as useful tools to investigate muscular work and gait dynamics. Multibody musculoskeletal simulations provide key insight into the biomechanics of the muscles and joints during movement, however it is difficult to predict a person's change in control strategy following an injury or neuromuscular disease. The symmetry of gait was also assessed in this review, with some researchers supporting the concept that normal walking is asymmetric. However, these asymmetries are small compared to asymmetry of gait behaviour resulting from injury, neuromuscular diseases, prosthetic feet or orthoses. For amputees, symmetrising the gait characteristics may not be the recommended method of rehabilitation [53]. A prosthetic foot has a different weight compared to an intact foot and this has a direct effect on the stance and swing dynamics of gait. The inability for a prosthetic foot or orthoses to mimic the contralateral muscular 
contributions mean that a healthy contralateral leg has to perform a different work pattern than usual, which may lead to further health problems.

Great insight may be gained by understanding asymmetric gait mechanics by using dynamic walking models. Merker et al. [95] used a bipedal spring-mass model to investigate asymmetries in touch-down angle, leg stiffness and system energy. They state that considerable differences between the contralateral legs can be tolerated and in some cases actually improve the robustness of the system dynamics. Rasouli et al. [111] use a passive dynamic walker with asymmetric leg mass positions claiming that asymmetric passive walkers are more sensitive to changes in mass ratio. Honeycutt et al. [55] also investigates asymmetric passive walkers but with changes in parameters such as mass, mass location, knee location and leg length of one leg. Their motivation is to analyse the passive dynamics of walking separate from cognitive control to test different gait rehabilitation hypotheses.

Research on using dynamic principles to understand asymmetric gait is lacking. Not only can this research into passive dynamics be expanded on further, but insight can also be gained by understanding the GRFs of asymmetric walking characteristics. Existing passive walking models do not simulate the human-like double-hump GRF (Figure 2-4), while spring-mass systems have not modelled individual leg masses and have not been used to investigate asymmetric leg inertias. The investigation into the effects of asymmetric gait dynamics to provide insight into gait rehabilitation is novel and is presented in this thesis. It is important to note that dynamic walking is only one of many possible approaches. These simple principles do not offer a solution to gait rehabilitation but these insights should be integrated into clinical observations and controlled experiments. 


\section{Chapter 3}

\section{Asymmetric passive dynamic walker}

As discussed in Section 2.2.2, a passive dynamic walker can traverse down a slope with remarkable human-like behaviour. With the COM following an inverted pendulum arc and the majority of energy transfer happening at the double-support, these dynamics can give us an insight into the underlying biomechanics of gait $[17,75]$. These models have sometimes been used to investigate asymmetric gait behaviour. Sushko et al. [137] altered the knee position of an asymmetric passive dynamic walker and showed that, contrary to traditional prosthetic designs, the prosthetic knee may not need to be in the same position as an intact knee to achieve optimal gait. Mahmoodi et al. [89] used a passive walker (PW) to in investigate the effect of roll-over shape on the characteristics of gait. Rasouli et al. [111] used a PW with asymmetric leg masses to study the chaotic and periodic behaviour. This chapter will focus on the effect of asymmetric leg conditions on the symmetry and stability of gait of the passive walking model. Close attention is given to the resultant gait characteristics and the effects an altered leg has on the healthy contralateral leg.

A passive dynamic walker model with incorporated rolling contact is used in this 
chapter to simulate bipedal walking dynamics. A circular foot shape has been shown to match the kinematics of the lower limb system $[45,2]$ and can mimic the trajectory of the COM during stance phase [94, 77]. The model set-up and derivations are explained in Section 3.1. Model parameters are selected to be close to 'normal' subject's gait characteristics in Section 3.2.1. Justification is made for adding a rocker to the end of the leg to simulate the roll-over kinematics. Comparisons are made with the point foot and walking with rocker feet in Section 3.3. In Section 3.4.1, a single leg's mass ratio and length ratio is altered to see the effect of asymmetrical inertial characteristics between the legs. In section 3.4.2 the effect of changing the roll-over shape's radius of curvature of one foot is investigated to see the effect this has on the dynamics of gait for the affected and unaffected legs.

\subsection{Equations of motion for the passive walker}

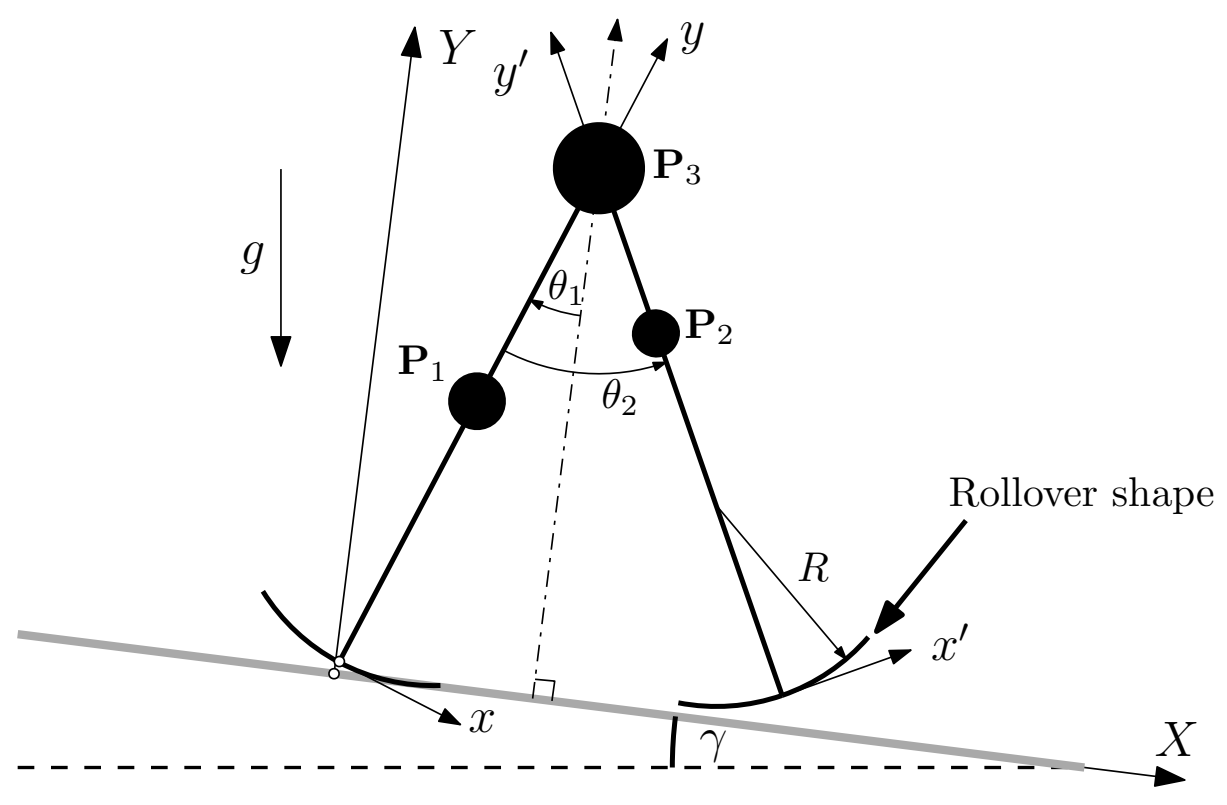

Figure 3-1: A schematic of the proposed smooth rolling passive walker with unbalanced masses.

The governing equations of the model consist of non-linear differential equations for the stance phase (Section 3.1.2) and algebraic equations for the double-support transition (Section 3.1.3). A schematic of the biped model is shown in Figure 3-1. The 
model's configuration can be described by $\theta_{1}$ being the angle made between the support leg with the normal to the ground and $\theta_{2}$, the angle between the support leg and the non-support swinging leg. The state vector $\boldsymbol{q}$ associated with the model is then

$$
\boldsymbol{q}=\left[\theta_{1}, \theta_{2}\right]^{T}
$$

The motion of the stance leg is determined by the roll-over shape, which has the form $y=f(x)$ in the reference frame defined $\{x, y\}$ in Figure 3-1. The analogous reference frame $\left\{x^{\prime}, y^{\prime}\right\}$ represents the non-support swinging leg. Let point mass $m_{1}$ be located at point $\mathbf{P}_{1}$ having fixed co-ordinates $\left[x_{1}, y_{1}\right]$ in the $\{x, y\}$ reference frame and point mass $m_{2}$ be located at point $\mathbf{P}_{2}$ having fixed co-ordinates $\left[x_{2}^{\prime}, y_{2}^{\prime}\right]$ in the $\left\{x^{\prime}, y^{\prime}\right\}$ reference frame. The third mass $m_{3}$ is located at the hip joint $\mathbf{P}_{3}$ at the

centre of rotation between the $\{x, y\}$ and $\left\{x^{\prime}, y^{\prime}\right\}$ reference frames and having the same co-ordinates, $\left[x_{3}, y_{3}\right]$ in the both frames. The global coordinates of each mass can be described by the $\{X, Y\}$ reference frame, aligned along the ground slope and its origin defined so that the point of contact with the floor, $s\left(\theta_{1}\right)$, is 0 when $\theta_{1}=0$.

\subsubsection{Rolling contact}

Roller feet provide better simulations of the inverted pendulum trajectory of the COM and progression of COP under the human foot during stance [47]. Figure 3-2 represents the location of COP. The point that the foot is in contact with the floor is defined by $x_{\theta}$ and $y_{\theta}$ in the coordinate system aligned with the stance leg, $\{x, y\}$ :

$$
\begin{aligned}
& x_{\theta}\left(\theta_{1}\right)=R \sin \left(\theta_{1}\right) \\
& y_{\theta}\left(\theta_{1}\right)=-R \cos \left(\theta_{1}\right)+R
\end{aligned}
$$

where $R$ is the radius of curvature of the rolling contact. In terms of the location of contact in the global coordinate system, $\{X, Y\}$, the point of contact with the ground 


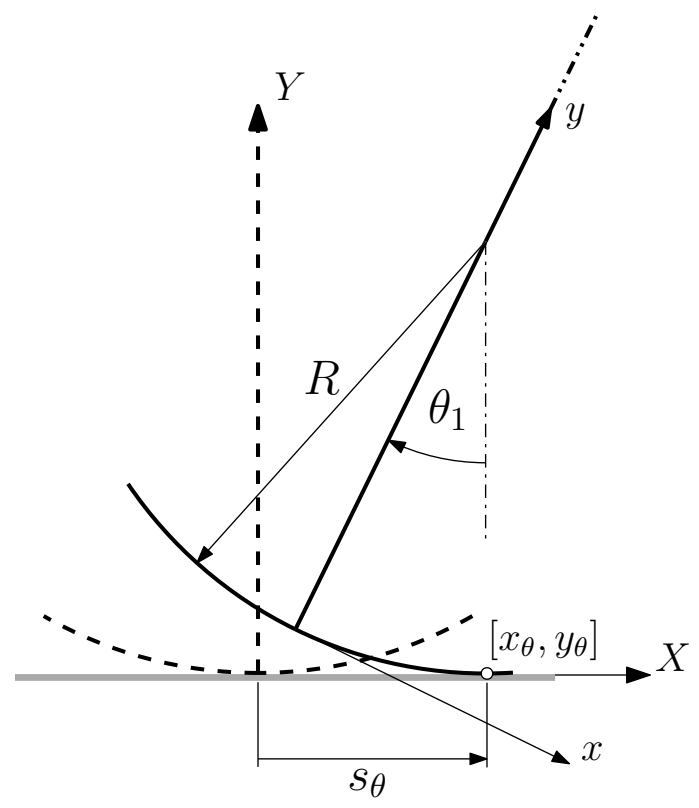

Figure 3-2: COP of the foot contact in the local coordinate system aligned with the leg is at $\left[x_{\theta}, y_{\theta}\right]$. The arc length is represented in the global coordinate system aligned with floor as $\left[s_{\theta}, 0\right]$.

is characterised by the arc-length of the foot,

$$
s_{\theta}(\theta)=R \theta
$$

\subsubsection{Stance phase}

The planar double-pendulum equations are adapted to include roll-over support for the foot contact. Its dynamical equations can be derived from the Euler-Lagrange approach:

$$
\frac{d}{d t}\left(\frac{\partial \mathcal{L}(\boldsymbol{q}, \dot{\boldsymbol{q}})}{\partial \dot{\boldsymbol{q}}}\right)-\frac{\partial \mathcal{L}(\boldsymbol{q}, \dot{\boldsymbol{q}})}{\partial \boldsymbol{q}}=0
$$

where the Lagrangian $\mathcal{L}(\boldsymbol{q}, \dot{\boldsymbol{q}})$ is the difference between kinetic and potential energies $\mathcal{L}(\boldsymbol{q}, \dot{\boldsymbol{q}})=K(\boldsymbol{q}, \dot{\boldsymbol{q}})-V(\boldsymbol{q})$. If the ground plane is at an angle, $\gamma$, with the horizontal plane $(\gamma<0$ if walking downhill as in Fig. 3-1) then the potential energy can be written with respect to a reference point which is defined as the point of COP when 
the stance leg is at an angle $\theta_{1}=0$ :

$V(\boldsymbol{q})=m_{1} g\left(X_{1} \sin \gamma+Y_{1} \cos \gamma\right)+m_{2} g\left(X_{2} \sin \gamma+Y_{2} \cos \gamma\right)+m_{3} g\left(X_{3} \sin \gamma+Y_{3} \cos \gamma\right)$

with $g$ being due to the gravitational effect and the positions for the points $\mathbf{P}_{1}$ and $\mathbf{P}_{3}$ are defined by the global coordinates

$$
\begin{aligned}
& X_{1}\left(\theta_{1}\right)=\left(x_{1}-x_{\theta}\left(\theta_{1}\right)\right) \cos \theta_{1}+\left(y_{1}-y_{\theta}\left(\theta_{1}\right)\right) \sin \left(\theta_{1}\right)+s\left(\theta_{1}\right) \\
& Y_{1}\left(\theta_{1}\right)=-\left(x_{1}-x_{\theta}\left(\theta_{1}\right)\right) \sin \theta_{1}+\left(y_{1}-y_{\theta}\left(\theta_{1}\right)\right) \cos \left(\theta_{1}\right) \\
& X_{3}\left(\theta_{1}\right)=\left(x_{3}-x_{\theta}\left(\theta_{1}\right)\right) \cos \theta_{1}+\left(y_{3}-y_{\theta}\left(\theta_{1}\right)\right) \sin \left(\theta_{1}\right)+s\left(\theta_{1}\right) \\
& Y_{3}\left(\theta_{1}\right)=-\left(x_{3}-x_{\theta}\left(\theta_{1}\right)\right) \sin \theta_{1}+\left(y_{3}-y_{\theta}\left(\theta_{1}\right)\right) \cos \left(\theta_{1}\right)
\end{aligned}
$$

where functions $x_{\theta}\left(\theta_{1}\right)$ and $y_{\theta}\left(\theta_{1}\right)$ represent the position of roll-over shape in contact with the floor and are defined with $[0,0]$ being at the end of the leg. Also for the swing leg $\mathbf{P}_{2}$,

$$
\begin{aligned}
& X_{2}\left(\theta_{1}, \theta_{2}\right)=\left(x_{2}\left(\theta_{2}\right)-x_{\theta}\left(\theta_{1}\right)\right) \cos \theta_{1}+\left(y_{2}\left(\theta_{2}\right)-y_{\theta}\left(\theta_{1}\right)\right) \sin \theta_{1}+s\left(\theta_{1}\right) \\
& Y_{2}\left(\theta_{1}, \theta_{2}\right)=-\left(x_{2}\left(\theta_{2}\right)-x_{\theta}\left(\theta_{1}\right)\right) \sin \theta_{1}+\left(y_{2}\left(\theta_{2}\right)-y_{\theta}\left(\theta_{1}\right)\right) \cos \theta_{1}
\end{aligned}
$$

where $\left[x_{2}, y_{2}\right]$ are coordinates of point $\mathbf{P}_{2}$ in the $\{x, y\}$ reference frame are defined by

$$
\begin{aligned}
& x_{2}\left(\theta_{2}\right)=\left(x_{2}^{\prime}-x_{3}\right) \cos \theta_{2}+\left(y_{2}^{\prime}-y_{3}\right) \sin \theta_{2}+x_{3} \\
& y_{2}\left(\theta_{2}\right)=-\left(x_{2}^{\prime}-x_{3}\right) \sin \theta_{2}+\left(y_{2}^{\prime}-y_{3}\right) \cos \theta_{2}+y_{3}
\end{aligned}
$$

The kinetic energy may be rewritten as

$$
K(\boldsymbol{q}, \dot{\boldsymbol{q}})=\frac{1}{2} m_{1}\left|\boldsymbol{v}_{1}\right|^{2}+\frac{1}{2} m_{2}\left|\boldsymbol{v}_{2}\right|^{2}+\frac{1}{2} m_{3}\left|\boldsymbol{v}_{3}\right|^{2}
$$


The vectors for velocities of each mass are given by:

$$
\begin{aligned}
& \overrightarrow{\boldsymbol{v}}_{1}=\dot{X}_{1} \vec{\imath}+\dot{Y}_{1} \vec{\jmath} \\
& \overrightarrow{\boldsymbol{v}}_{2}=\dot{X}_{2} \vec{\imath}+\dot{Y}_{2} \vec{\jmath} \\
& \overrightarrow{\boldsymbol{v}}_{3}=\dot{X}_{3} \vec{\imath}+\dot{Y}_{3} \vec{\jmath}
\end{aligned}
$$

By inserting Equations (3.12), (3.13) and (3.14) into (3.11), $K(\boldsymbol{q}, \dot{\boldsymbol{q}})$ can be rewritten as:

$$
K(\boldsymbol{q}, \dot{\boldsymbol{q}})=\frac{1}{2} \dot{\boldsymbol{q}}^{T} \boldsymbol{M}(\boldsymbol{q}) \dot{\boldsymbol{q}}
$$

or expanding to separate out $\dot{\theta}_{1}$ and $\dot{\theta}_{2}$

$$
K(\boldsymbol{q}, \dot{\boldsymbol{q}})=\frac{1}{2} M_{11} \dot{\theta}_{1}^{2}+\frac{1}{2} M_{22} \dot{\theta}_{2}^{2}+M_{12} \dot{\theta}_{1} \dot{\theta}_{2}
$$

where the values for $M_{i j}$ can be found from:

$$
M_{i j}=\sum_{m=1}^{3} m_{m}\left(\frac{\partial X_{m}}{\partial q_{i}} \frac{\partial X_{m}}{\partial q_{j}}+\frac{\partial Y_{m}}{\partial q_{i}} \frac{\partial Y_{m}}{\partial q_{j}}\right)
$$

The equations of motion, derived from the Lagrangian (Equation (3.5)), can then be described as

$$
\begin{aligned}
& \boldsymbol{M}(\boldsymbol{q}) \ddot{\boldsymbol{\theta}}+\boldsymbol{N}(\boldsymbol{q}, \dot{\boldsymbol{q}}) \dot{\boldsymbol{q}}+\boldsymbol{V}^{\prime}(\boldsymbol{q})=0 \\
& \boldsymbol{M}(\boldsymbol{q})=\left[\begin{array}{ll}
M_{11} & M_{12} \\
M_{21} & M_{22}
\end{array}\right] \\
& \boldsymbol{N}(\boldsymbol{q}, \dot{\boldsymbol{q}})=\left[\begin{array}{ll}
N_{11} & N_{12} \\
N_{21} & N_{22}
\end{array}\right] \\
& \boldsymbol{V}^{\prime}(\boldsymbol{q})=\left[\begin{array}{ll}
\frac{\partial V}{\partial \theta_{1}} & \frac{\partial V}{\partial \theta_{2}}
\end{array}\right]^{T}
\end{aligned}
$$

with

$$
N_{i j}=\sum_{n=1}^{2} \frac{\partial M_{i n}}{\partial q_{j}} \dot{q}_{n}-\frac{1}{2} \sum_{n=1}^{2} \frac{\partial M_{j n}}{\partial q_{i}} \dot{q}_{n}
$$




\subsubsection{The double support transition phase}

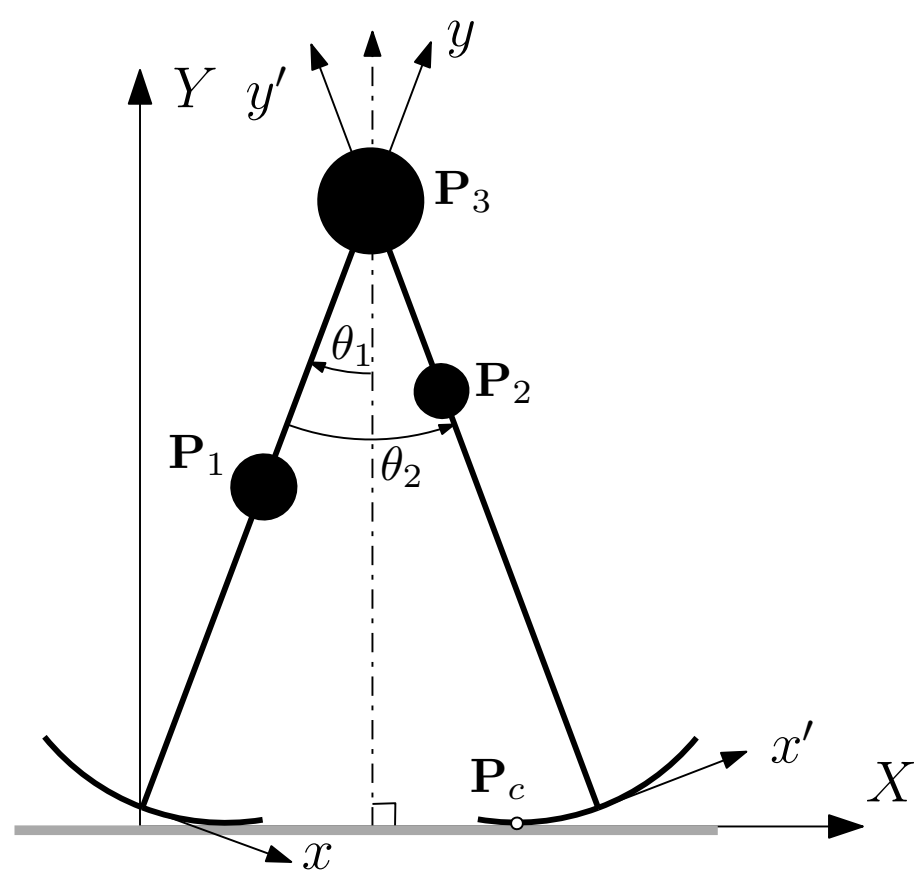

Figure 3-3: Orientation of the biped walker at double-support transition pre-impact.

The transition equations for the point foot model have been outlined previously by McGeer [94] and Goswami et al. [40]. The PW model has rigid, non-elastic legs and the impact with the ground is assumed to be instantaneous. At the double-support phase the COM momentum changes direction. Heel strike occurs when the swing leg touches the floor and the following conditions are met:

- The swing leg is travelling backwards at the initial contact $-\dot{\theta}_{2}$ is positive at heel strike.

- The swing leg is in front and has passed the stance leg $-\theta_{2}$ is negative.

The contact made with the floor by the swinging leg results in an instantaneous impact with no slipping. As the body configuration remains unchanged during impact, the support leg is switched to the front leg using the function

$$
\boldsymbol{q}^{+}=\boldsymbol{J} \boldsymbol{q}^{-}
$$


where the superscripts ${ }^{-}$and ${ }^{+}$indicate respectively pre-impact and post-impact variables and

$$
\boldsymbol{J}=\left[\begin{array}{cc}
1 & 1 \\
0 & -1
\end{array}\right]
$$

The point at which the swing leg makes contact with the floor is donated by $\mathbf{P}_{c}$ as shown in Figure 3-3, having coordinates $\left[X_{c}, 0\right]$ at heel-strike. The position of $\mathbf{P}_{c}$ is given by

$$
\begin{aligned}
& X_{c}=\left(x_{c}-x_{\theta}\right) \cos \theta_{1}+\left(y_{c}-y_{\theta}\right) \sin \theta_{1}+s\left(\theta_{1}\right) \\
& Y_{c}=-\left(x_{c}-x_{\theta}\right) \sin \theta_{1}+\left(y_{c}-y_{\theta}\right) \cos \theta_{1} \\
& x_{c}=\left(x_{\theta_{2}}-x_{3}\right) \cos \theta_{2}+\left(y_{\theta_{2}}-y_{3}\right) \sin \theta_{2}+x_{3} \\
& y_{c}=-\left(x_{\theta_{2}}-x_{3}\right) \sin \theta_{2}+\left(y_{\theta_{2}}-y_{3}\right) \cos \theta_{2}+y_{3}
\end{aligned}
$$

with $s\left(\theta_{1}\right)$ given by Equation (3.4). Let $\mathbf{P}_{0}$ be the origin $[0,0]$ of the next step in the $\{X, Y\}$ frame and

$$
\mathbf{P}_{0}=\mathbf{P}_{c}-s_{2}
$$

with $s_{2}$ being the arc-length of the swing leg the collision.

Although for simplicity, the transition stage is assumed to be instantaneous, it must be remembered that the hind leg can only leave the ground after the front leg has made contact and is supporting the walker. During the transfer there is an completely inelastic collision in which support transfers from one leg to the other. Derived from the rimless wheel mechanics by McGeer [94], the system must conserve the following conditions at collision:

- Angular momentum of the whole system about the point of collision.

- Angular momentum of the trailing leg about the hip joint.

Equations (3.28) and (3.29) are obtained by conserving angular momentum, first from each mass about the collision point $\mathbf{P}_{c}$ and then at the trailing leg about the hip joint 
$\mathbf{P}_{3}$. The coordinates are taken from the leading leg.

$$
\begin{aligned}
m_{1} \overrightarrow{\mathbf{P}_{c} \mathbf{P}_{1}} \times \boldsymbol{v}_{1}^{-}+m_{2} \overrightarrow{\mathbf{P}_{c} \mathbf{P}_{2}} & \times \boldsymbol{v}_{2}^{-}+m_{3} \overrightarrow{\mathbf{P}_{c} \mathbf{P}_{3}} \times \boldsymbol{v}_{3}^{-} \\
& =m_{1} \overrightarrow{\mathbf{P}_{c} \mathbf{P}_{1}} \times \boldsymbol{v}_{1}^{+}+m_{2} \overrightarrow{\mathbf{P}_{c} \mathbf{P}_{2}} \times \boldsymbol{v}_{2}^{+}+m_{3} \overrightarrow{\mathbf{P}_{c} \mathbf{P}_{3}} \times \boldsymbol{v}_{3}^{+} \\
& m_{1} \overrightarrow{\mathbf{P}_{3} \mathbf{P}_{2}} \times \boldsymbol{v}_{1}^{-}=m_{1} \overrightarrow{\mathbf{P}_{3} \mathbf{P}_{2}} \times \boldsymbol{v}_{1}^{+}
\end{aligned}
$$

With the vectors given by:

$$
\begin{aligned}
& \overrightarrow{\mathbf{P}_{c} \mathbf{P}_{1}}=\left(X_{1}^{+}-s\left(\theta_{1}^{+}\right)\right) \vec{\imath}+Y_{1} \vec{\jmath} \\
& \overrightarrow{\mathbf{P}_{c} \mathbf{P}_{2}}=\left(X_{2}^{+}-s\left(\theta_{1}^{+}\right)\right) \vec{\imath}+Y_{2} \vec{\jmath} \\
& \overrightarrow{\mathbf{P}_{c} \mathbf{P}_{3}}=\left(X_{3}^{+}-s\left(\theta_{1}^{+}\right)\right) \vec{\imath}+Y_{3} \vec{\jmath} \\
& \overrightarrow{\mathbf{P}_{3} \mathbf{P}_{1}}=\left(X_{1}^{+}-X_{3}^{+}\right) \vec{\imath}+\left(Y_{1}-Y_{3}\right) \vec{\jmath}
\end{aligned}
$$

and the superscripts ${ }^{-}$and ${ }^{+}$indicate respectively pre-impact and post-impact variables. At post impact, $\mathbf{P}_{1}$ becomes the swinging leg and $\mathbf{P}_{2}$ becomes the stance leg. The conservation of angular momentum leads to the condition

$$
\dot{\boldsymbol{q}}^{+}=\frac{\boldsymbol{Q}^{-}(\theta)}{\boldsymbol{Q}^{+}(\theta)} \dot{\boldsymbol{q}}^{-}
$$

with the pre-impact transfer matrix $\boldsymbol{Q}^{-}$being

$$
\begin{aligned}
Q_{11}^{-} & \left.=m_{1}\left(\frac{\partial Y_{1}}{\partial \theta_{1}^{-}}\left(X_{1}^{+}-s^{+}\right)\right)-\frac{\partial X_{1}}{\partial \theta_{1}^{-}} Y_{1}\right) \\
& +m_{2}\left(\frac{\partial Y_{2}}{\partial \theta_{1}^{-}}\left(X_{2}^{+}-s^{+}\right)-\frac{\partial X_{2}}{\partial \theta_{1}^{-}} Y_{2}\right) \\
& +m_{3}\left(\frac{\partial Y_{3}}{\partial \theta_{1}^{-}}\left(X_{3}^{+}-s^{+}\right)-\frac{\partial X_{3}}{\partial \theta_{1}^{-}} Y_{3}\right) \\
Q_{12}^{-} & =m_{2}\left(\frac{\partial Y_{2}}{\partial \theta_{2}^{-}}\left(X_{3}^{+}-s^{+}\right)-\frac{\partial X_{2}}{\partial \theta_{2}^{-}} Y_{2}\right) \\
Q_{21}^{-} & =m_{1}\left(X_{3} X_{1 p r e} \frac{\partial Y_{1}}{\partial \theta_{1}^{-}}-Y_{3} \vec{Y}_{1 p r e} \frac{\partial X_{1}}{\partial \theta_{1}^{-}}\right) \\
Q_{22}^{-} & =0
\end{aligned}
$$


and for the post-impact transfer matrix $\boldsymbol{Q}^{-}$,

$$
\begin{aligned}
Q_{11}^{+} & =m_{1}\left(\frac{\partial Y_{1}^{+}}{\partial \theta_{1}^{+}}\left(X_{1}^{+}-s^{+}\right)-\frac{\partial X_{1}^{+}}{\partial \theta_{1}^{+}} Y_{1}\right) \\
& +m_{2}\left(\frac{\partial Y_{2}}{\partial \theta_{1}^{+}}\left(X_{2}^{+}-s^{+}\right)-\frac{\partial X_{2}}{\partial \theta_{1}^{+}} Y_{2}\right) \\
& +m_{3}\left(\frac{\partial Y_{3}^{+}}{\partial \theta_{1}^{+}}\left(X_{3}^{+}-s^{+}\right)-\frac{\partial X_{3}^{+}}{\partial \theta_{1}^{+}} Y_{3}^{+}\right) \\
Q_{12}^{+} & =m_{1}\left(\frac{\partial Y_{2}}{\partial \theta_{2}^{+}}\left(X_{2}^{+}-s^{+}\right)-\frac{\partial X_{2}}{\partial \theta_{2}^{+}} Y_{2}\right) \\
Q_{21}^{+} & =m_{1}\left(\overrightarrow{X_{3} X_{1 p o s t}} \frac{\partial Y_{2}}{\partial \theta_{1}^{+}}-Y_{3} Y_{1 \text { post }} \frac{\partial X_{2}}{\partial \theta_{1}^{+}}\right) \\
Q_{22}^{+} & =m_{1}\left(\overrightarrow{X_{3} X_{1 \text { post }}} \frac{\partial Y_{2}}{\partial \theta_{2}^{+}}-\vec{Y}_{3} Y_{1 \text { post }} \frac{\partial X_{2}}{\partial \theta_{2}^{+}}\right)
\end{aligned}
$$

\subsubsection{Numerical implementation}

To solve the stance-phase dynamics outlined in Equation 3.18, the MATLAB ode45 function is implemented to solve the ordinary differential equations $[\dot{\boldsymbol{q}}, \ddot{\boldsymbol{q}}]$ using the Runge-Kutta method. The collision event is enabled when the touch-down conditions are met and $\mathbf{P}_{c}=0$. A diagram of the order of a single walking step can be seen in Figure 3-4. Before results are evaluated, the model is given a random set of initial conditions $\boldsymbol{q}_{0}$. If the walking model falls over in under 200 steps, a different set of random initial conditions is given. This continues until the model reaches a stable walking solution (defined in Section 3.2.2) or no stability is found within 500 attempts. Figure 3-5 demonstrated a flowchart indicating the algorithm implemented.

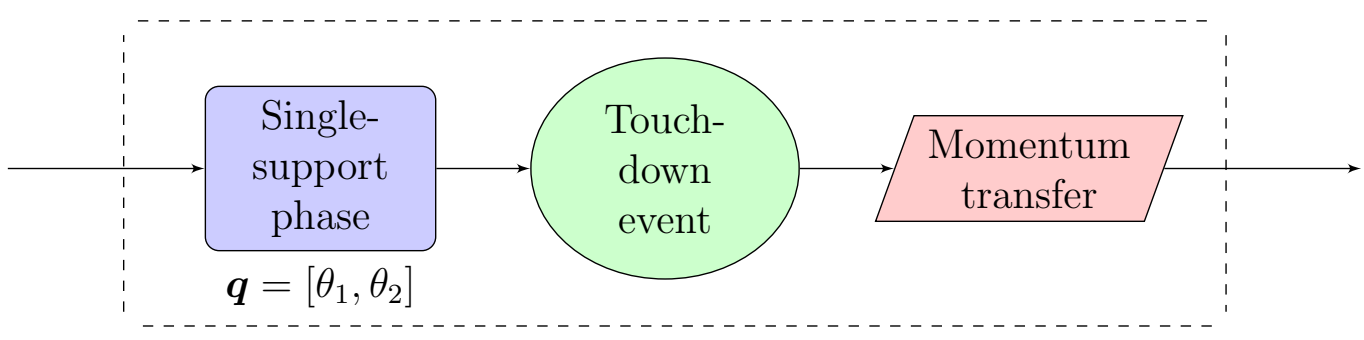

Figure 3-4: Flowchart of a complete walking step in the PW model. Can be integrated as the 'Complete Walking Step' block in Figure 3-5. 


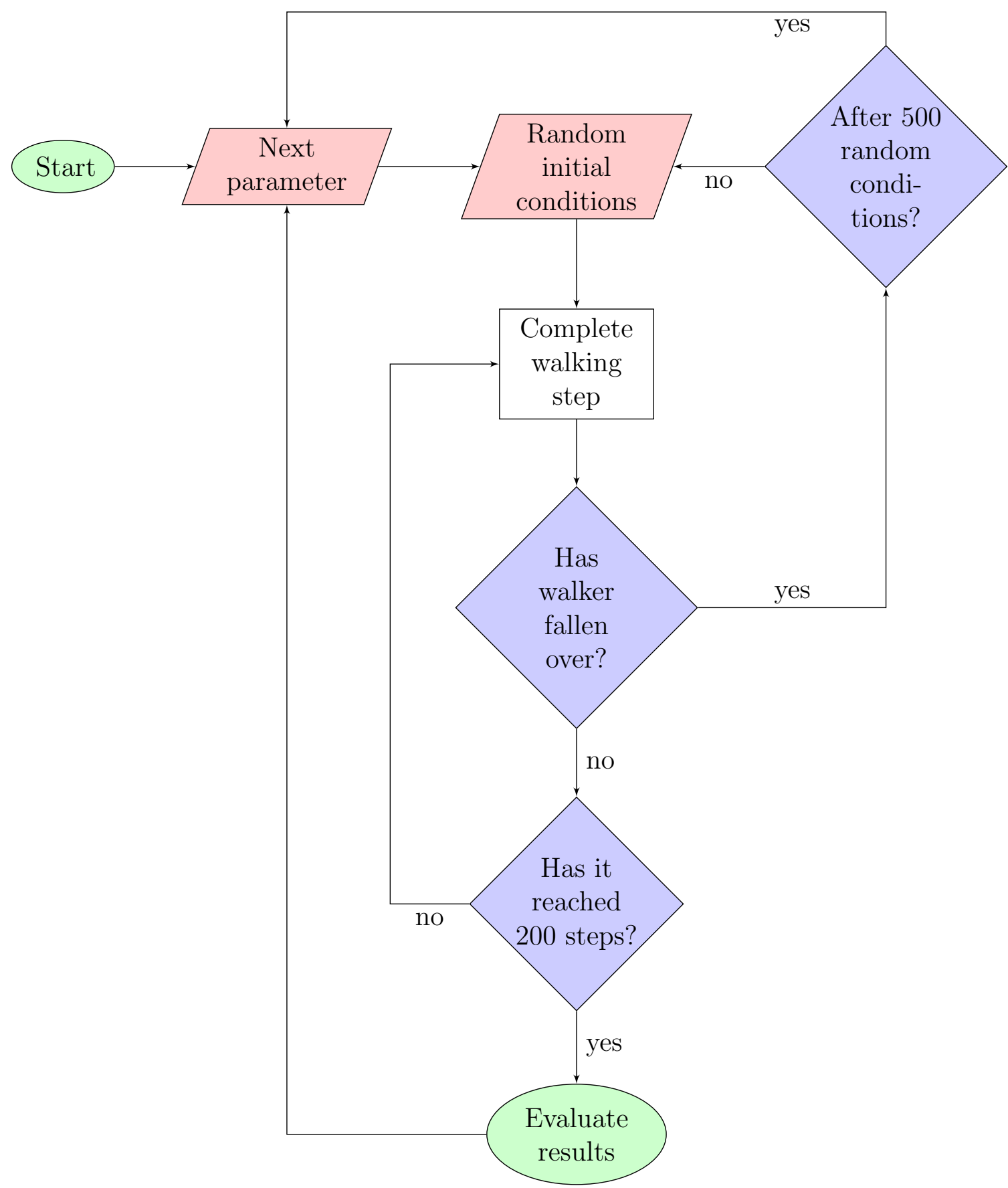

Figure 3-5: Flowchart of the algorithm used to plot bifurcation diagrams. One parameter is altered independently. If the walker does not reach a stable solution of 200 steps within 500 attempts, the initial set of parameters is seen as a 'failure' and a cycle with the next input parameter is attempted. 


\subsubsection{Validation of results}

The model can be set up the same as the compass walker in research from Goswami et al. [41]. A comparison between the two models is seen in Figure 3-6. An extra chaotic gait is seen in the current PW model at a slope of $4^{\circ}$. This nature was not captured in the model from Goswami et al. [41] however this type of nature has been noted in previous research [79] and is out of the scope for this thesis.

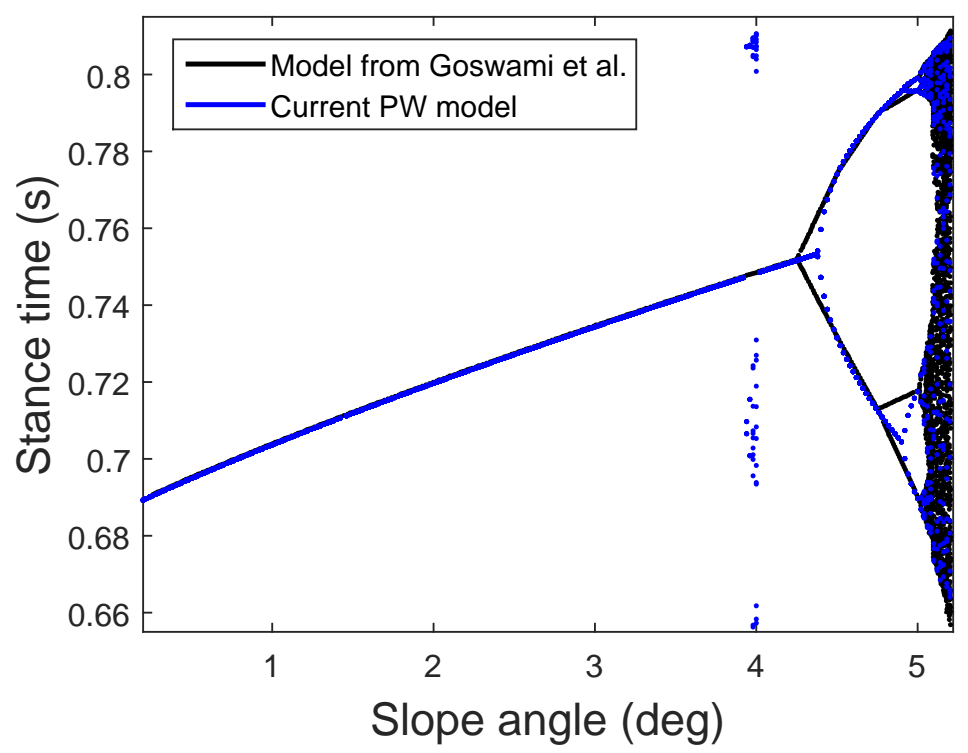

Figure 3-6: Comparison between the current PW model results (blue) and the results obtained from Goswami et al. [41] (black). Model parameters are mass ratio $\mu=2$ and length ratio $\beta=1$ (see Section 3.2.1). The parameter being altered in these bifurcation diagrams is the angle of the ground decline, $\gamma$.

\subsection{Understanding results}

\subsubsection{Values used for parameters}

The dynamics of the model during stance phase is comparable to a double-inverted

pendulum and has four states $\theta_{1}(t), \theta_{2}(t), \dot{\theta}_{1}(t)$ and $\dot{\theta}_{2}(t)$. To compare these results 
to clinical observational data, model parameters are arbitrarily selected to be close to the human locomotor system.

The model is comprised of a mass concentrated at the hip and a smaller mass placed along each leg. The coordinates of the foot contact is represented as a function of the leg angle $x_{\theta}\left(\theta_{1}\right), y_{\theta}\left(\theta_{1}\right)$ (derived from roller radius in Appendix 3.1.1) which allows for flexibility for the shape of the foot. The foot shape may be represented as a polynomial function with a defined heel length and forefoot length [89, 12], however in this section, the radius of curvature of the foot is taken as $30 \%$ of the leg length $[94,45]$ to match empirical data of the human system. To normalise results for the walking model, the mass ratio is defined,

$$
\mu=\frac{\text { Hip mass }}{\operatorname{Leg} \text { mass }}=\frac{m_{H}}{m_{A}}
$$

and leg mass position ratio

$$
\beta=\frac{\text { Upper leg length }}{\text { Lower leg length }}=\frac{y_{H}-y_{A}}{y_{A}} .
$$

can be rearranged this to obtain the location of leg mass in terms of coordinates $[x, y]$,

$$
y_{A}=\frac{y_{H}}{\beta+1}
$$

To have a comparison between the model dynamics and human walking, anatomical parameters are used. Table 3.1 outlines the standard values for the parameters used. Mahmoodi [88] has derived these dimensionless values for light/medium/heavy persons from anthropomorphic data obtained by Ohio [105].

\subsubsection{Limit cycles and a complete walking step}

If given a set of unstable initial conditions, the model will fall over and not complete a step cycle. In this model the swing leg does not retract; it is assumed that the foot 


\begin{tabular}{lcc}
\hline Name & Notation & Value \\
\hline Leg length & $L$ & $1 \mathrm{~m}$ \\
Total mass & $\sum m$ & $80 \mathrm{~kg}$ \\
Length ratio & $\beta$ & 0.6 \\
Mass ratio & $\mu$ & 3.6 \\
Radius of gyration/leg length & $R$ & 0.3 \\
Slope angle & $\gamma$ & $2^{\circ}$ \\
\hline
\end{tabular}

Table 3.1: Standard parameters used for the PW model.

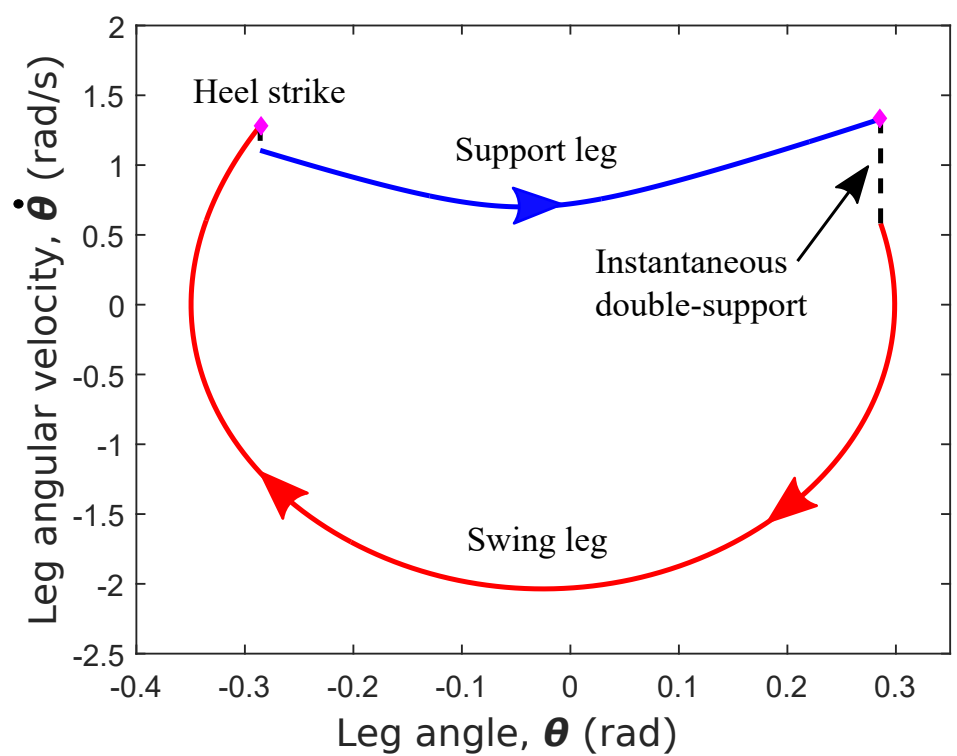

Figure 3-7: Schematic to show how the phase-plane of $\boldsymbol{q}$ evolves over a walking step. The blue line represents the support leg, while the red line shows the evolution of the swing leg. The diamonds represents the point at which a heel-strike condition occurs and the conditions 'jump' as the walker reaches another step.

does not scuff on the floor and contact is ignored as the swing leg moves forward. A successful walking step is reached if the swing leg is in front of the support leg $\left(\theta_{2}<0\right)$ and is retracting $\left(\dot{\theta}_{2}>0\right)$. An immediate failure occurs if the model falls (the hip mass touches the ground).

The schematic diagram as shown in Figure 3-7 describes the phase-plane from an average walking step. The angle of the support leg increases as COM moves forward and at heel-strike, a completely inelastic collision occurs in a negligible time frame as the walker loses mechanical energy at the transition. After a few walking steps, 
the model is considered to be 'stable', at which point it may have a symmetric or n-period gait.

Periodic solutions exist for the state of $\boldsymbol{q}(t)=(\boldsymbol{\theta}(t), \dot{\boldsymbol{\theta}}(t))$. These orbits in space state are known as limit cycles, which correspond to the Poincaré return map. As can be seen in Figure 3-8, the walker converges to a steady gait from a random set of initial conditions. As the slope angle $\gamma$ increases, the walking model exhibits perioddoubling [125], in which the walker converges to a steady asymmetric gait, as one leg has a different phase-plot than the other. Figures 3-8d and 3-8f demonstrate a 2-period and 4-period gait respectively. For practical reasons, the walker takes 100 steps and the last 32 steps are plotted, so that steady, stable walking is reached and a symmetry of up to 32-period may be seen. Figure 3-9 shows the inter-leg angle at heel-strike in respect to an increasing slope angle, $\gamma$. As the slope is increased further, these bifurcations increase to chaotic walking as is highlighted in Figure 3-9c. This bifurcation can also be seen from changing other gait parameters such as mass ratio and length ratio [40, 41, 154].

\subsection{Point foot vs. curved foot}

The simple compass walker model usually has a point foot [37]. Humans tend to walk on a roll-over shape or effective rocker radius of about $30 \%$ the length of the leg $[94,45,2]$, however few models use this as the foot contact. Figure 3-10 outlines a summary of the gait characteristics of both models as the slope angle, $\gamma$, increases. From the comparison of gait parameters of the two models in Figure 3-10, the curved foot seems more robust - capable of reaching a larger slope angle (energy input) and a higher walking velocity. Although point feet may be used for model simplicity, these results suggest that the rolling contact foot has a significant effect on the gait of the model.

For the results in this section there are no limits on the arc length of rolling contact 


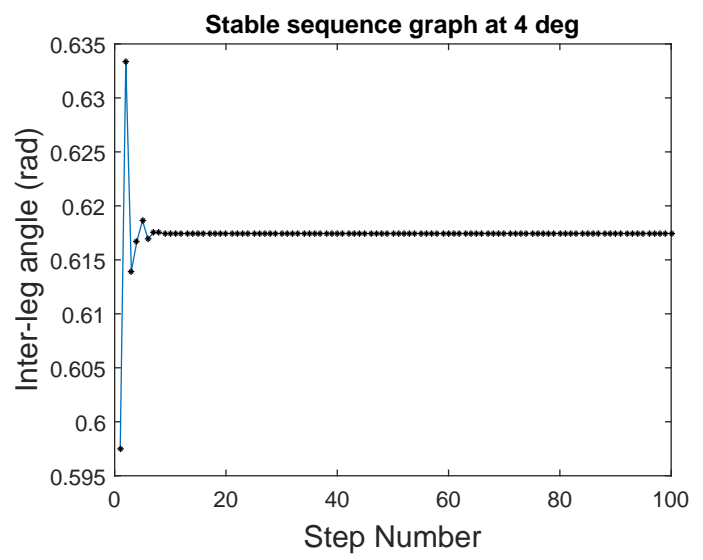

(a)

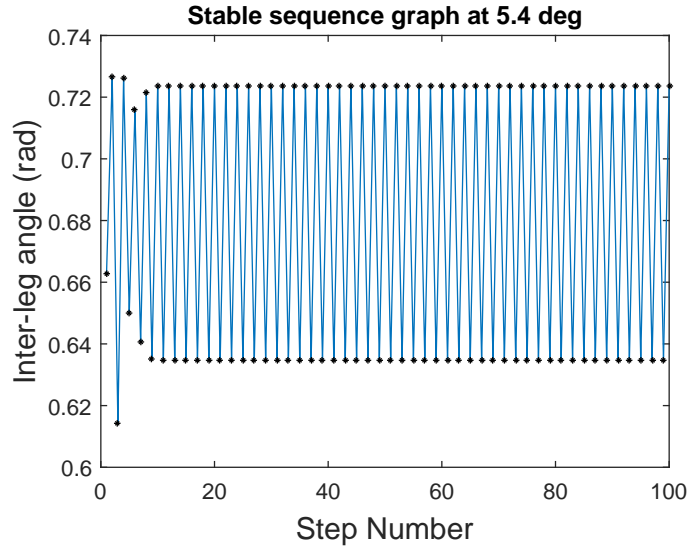

(c)

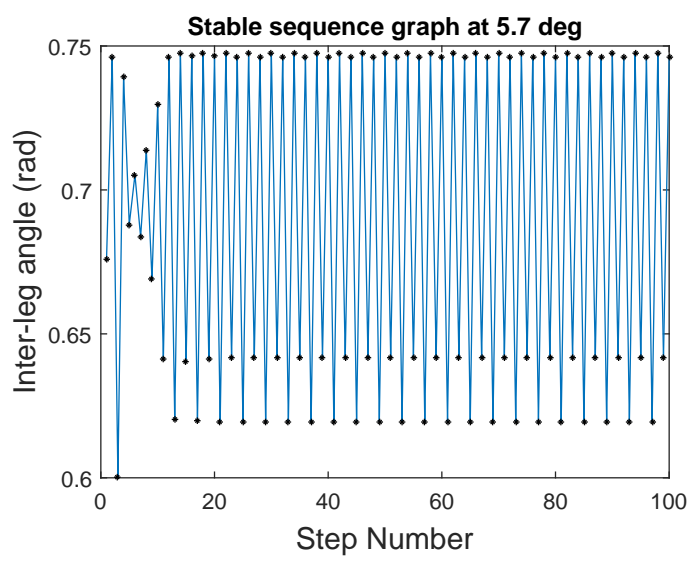

(e)

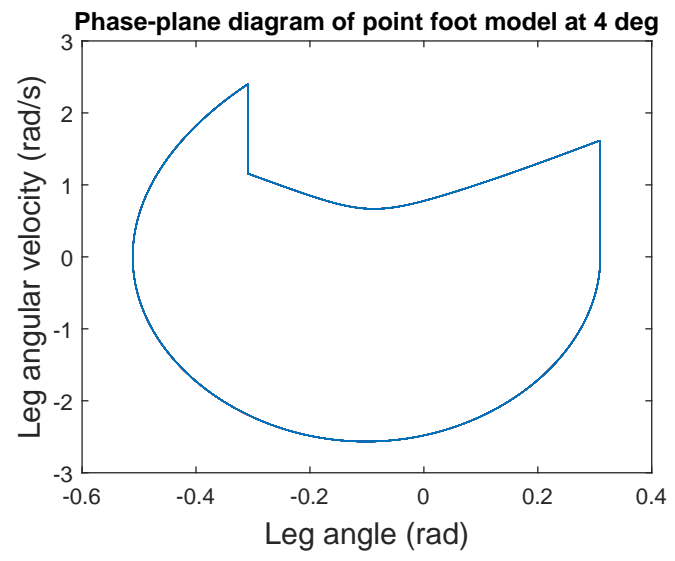

(b)

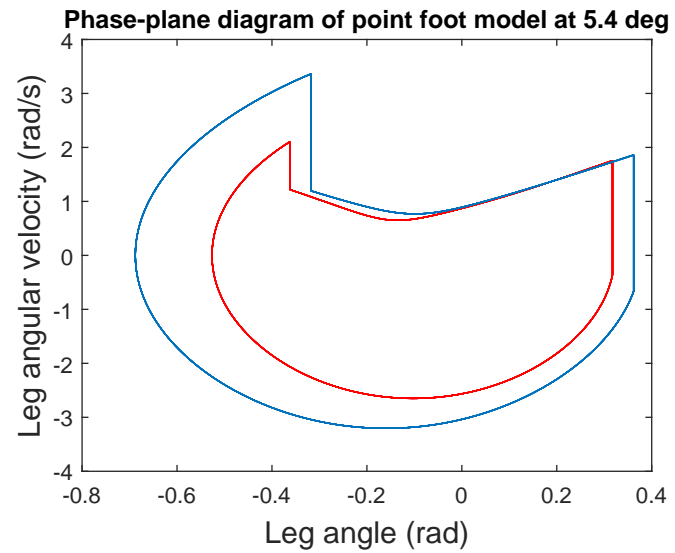

(d)

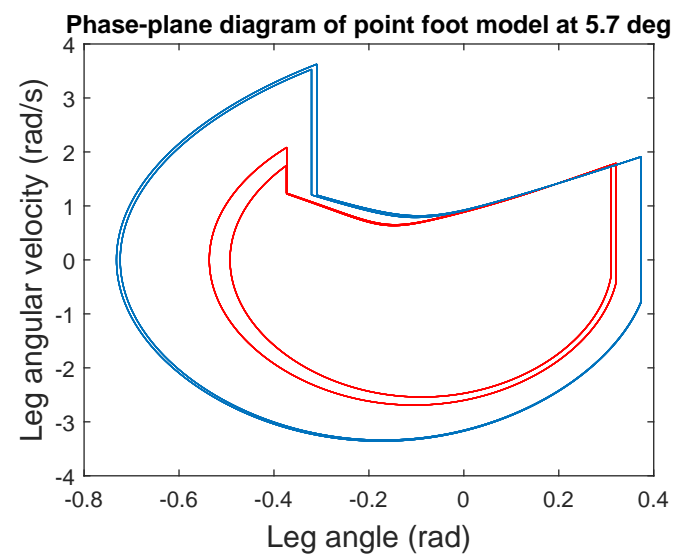

(f)

Figure 3-8: Periodic limit cycles of the point foot model at a slope angle of 4, 5.4 and 5.7 degrees with the phase plane diagrams (right) plotted for the last 32 steps. Plots on the left show that stable gait is achieved within a few walking steps where each dot represents $\theta_{2}$ at the end of the step. Phase-plane limit cycles of the left leg (red) and right leg (blue) show that symmetric periodicity can be seen. 


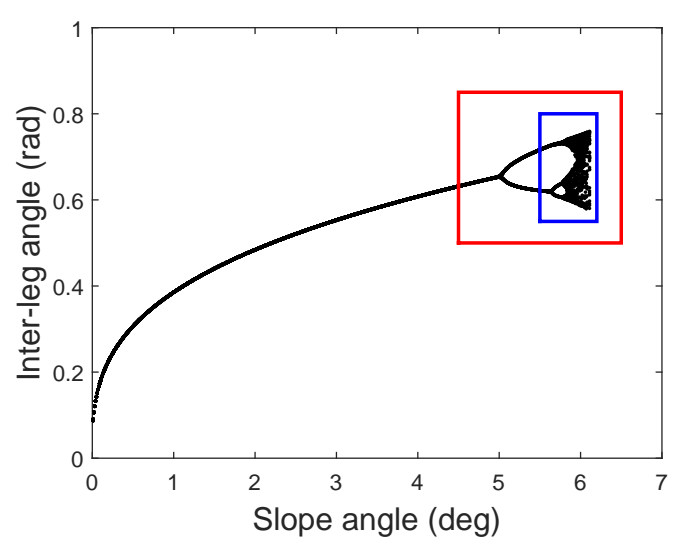

(a)

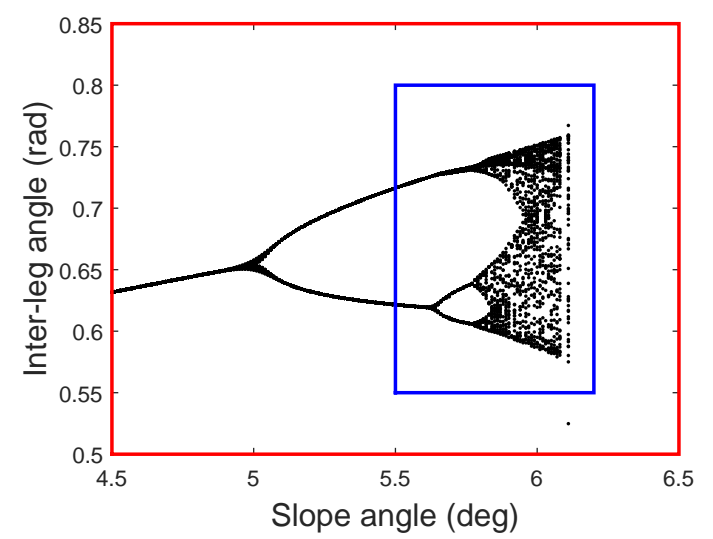

(b)

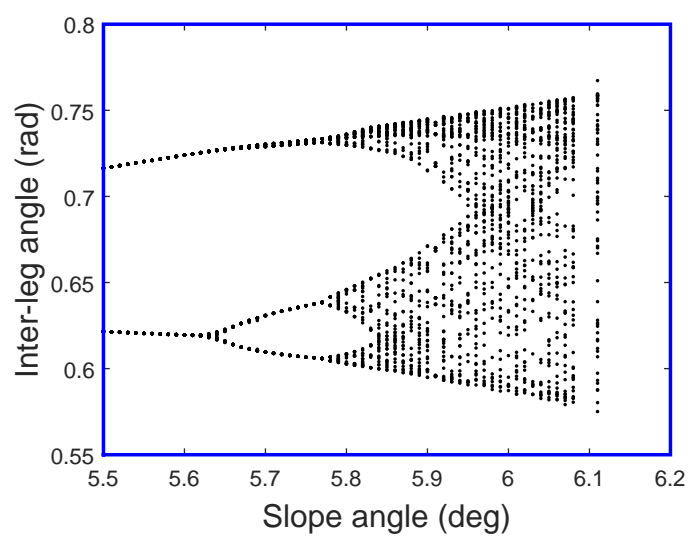

(c)

Figure 3-9: Bifurcation diagram of the point foot contact zoomed in. The red square shown in (a) is stretched to plot (b) and the blue box in Figures (a) and (b) is stretched to obtain plot (c).

used, however this may be worth investigating in future research. It is worth noting that due to there being no limit to the foot length, $\theta_{2}$ remains equal to twice $\theta_{1}$ at the double-support phase due to geometric constraints. This property is eliminated if a heel length and forefoot length is added, however the contact angle $\theta_{2}$ can still be found as a function of $\theta_{1}$ with the double-support constraint.

\subsubsection{Gait characteristics}

The solution $\boldsymbol{q}$ for each step can be evaluated by its components $\theta_{1}(t), \theta_{2}(t), \dot{\theta}_{1}(t) \&$ $\dot{\theta}_{2}(t)$. Gait can be evaluated using spatial and temporal parameters such as inter-leg 


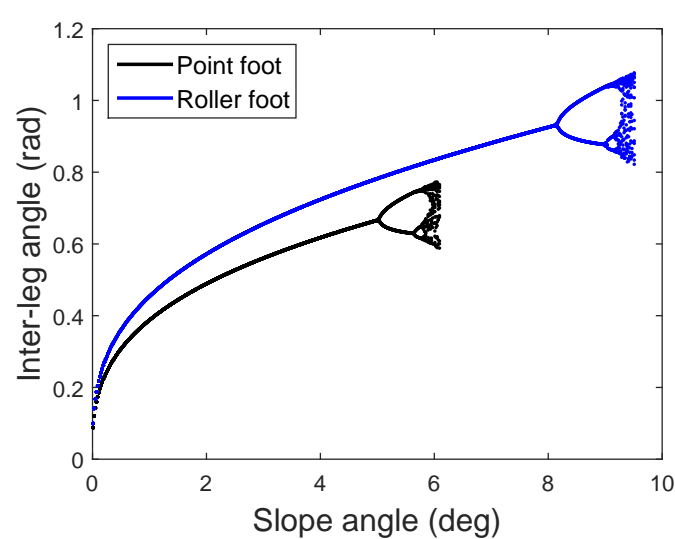

(a)

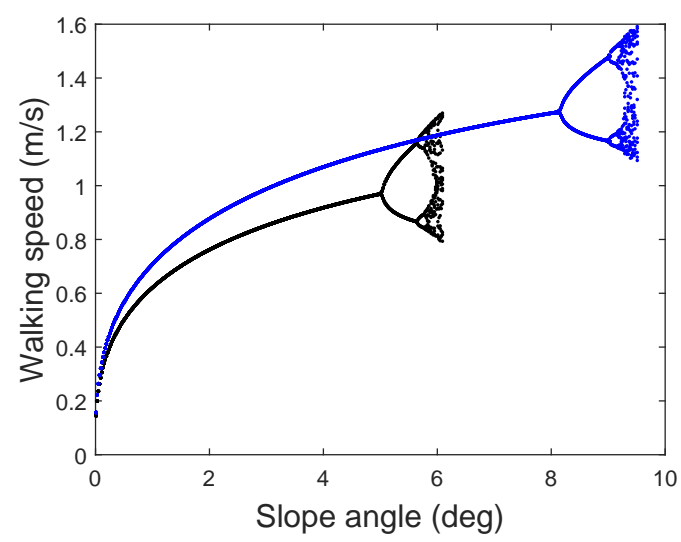

(c)

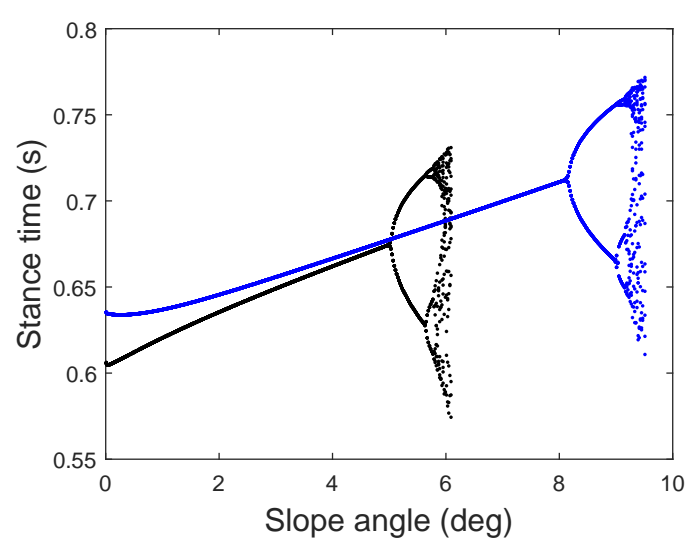

(b)

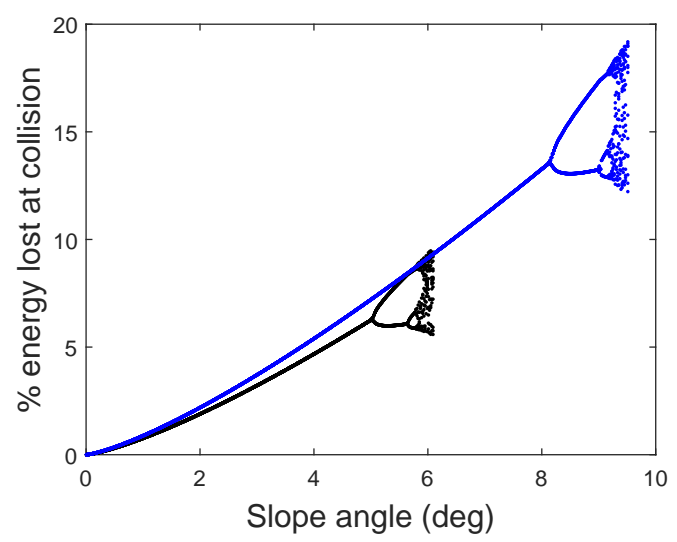

(d)

Figure 3-10: Bifurcation diagram comparisons between point foot (black) and roller foot (blue) PW models as a function of slope angle, $\gamma$. The curved foot has a radius of $30 \%$ of the leg length.

angle (analogous to step length), stance time (from heel-strike to opposite heel-strike) and walking speed [54]. The passive compass walking model can also predict the energy lost at the double-support to redirect the COM [24]. These gait parameters help to differentiate the different gait dynamics of each step and relate to human walking characteristics. The sub-plots in Figures 3-12, 3-13 \& 3-14 show the resulting gait characteristics; (a) Inter-leg angle, $\theta_{2}$ at heel-strike; (b) The time taken between each step; (c) The average walking speed of each step; (d) The ratio of mechanical energy lost during the collision at the end of the step. This is discussed in detail in the next section. 


\subsection{Investigating asymmetry in the passive walker}

Injury or disability may cause asymmetric walking and alter whole body gait patterns. In this section, the dynamic effects of changing only one leg (see Figure 3-11) will be investigated to see how this affects the gait characteristics and the unmodified contralateral leg. Real world applications may be to investigate the dynamic effects of mass imbalance due to rehabilitative devices such as prosthetic feet, orthotics or exoskeletons. Symmetric leg function is not necessarily the aim of rehabilitation [53], however these results will help distinguish how gait characteristics are affected by loading conditions or alterations in the effective roll-over shape for the COM trajectory during stance.

\subsubsection{Asymmetric leg masses}

A mass imbalance of both legs is common for prosthetic feet and other devices that affect the weight of the lower leg. Both the quantity and position of the COM of the leg will have an effect on the mechanics of gait during the stance phase and swing phase. This section will investigate, in particular, the effects of an asymmetric leg mass ratio $\mu$ and leg $\mathrm{COM}$ position ratio $\beta$.

The effect of variation of mass ratios and leg ratios has been investigated before in symmetric passive walkers [40], however there have been few published results on the effect of asymmetric masses on these models [55]. It is the objective of this research to quantify the effects of these asymmetries on a range of gait characteristics.

\section{Asymmetric leg mass ratio, $\mu$}

The mass ratio $\mu$ of one leg $\left(\mu_{B}=m_{H} / m_{B}\right)$ is changed, while the other leg remains at a constant mass ratio, $\mu_{B}$, of 3.6. In a standard pendulum model (without stepto-step transitions), the quantity of mass will not have an effect on the gait period, 


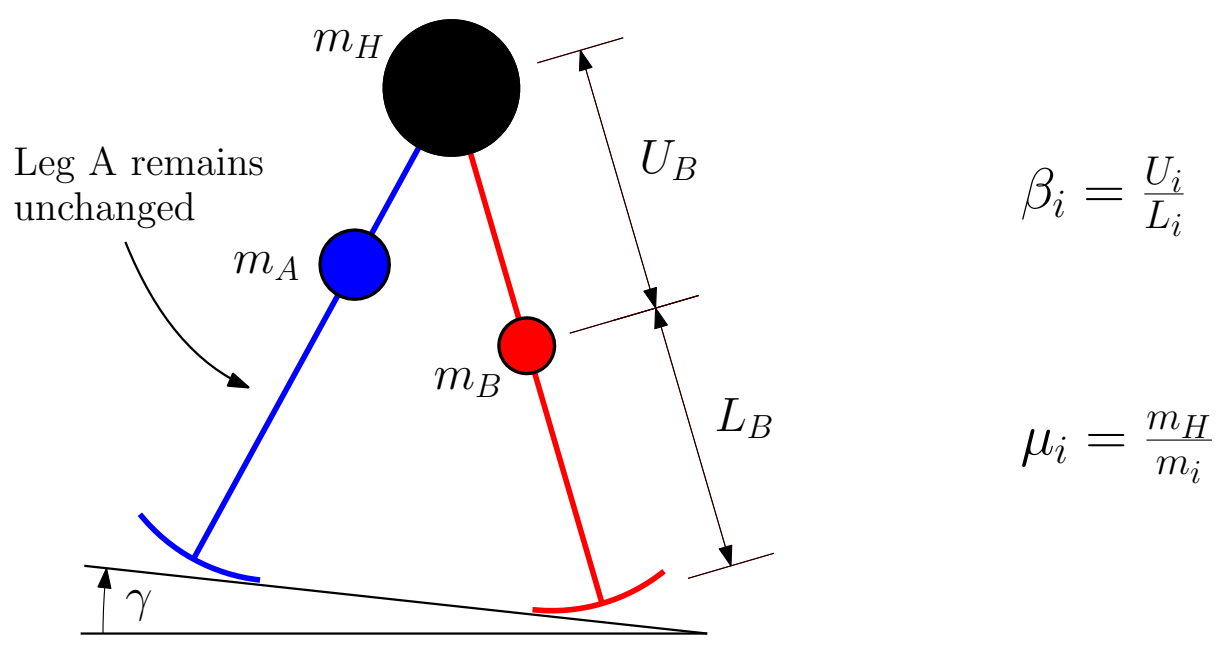

Figure 3-11: Length ratio $\left(\beta_{A}=\frac{U_{A}}{L_{A}}\right)$ and mass ratio $\left(\mu_{A}=\frac{m_{H}}{m_{A}}\right)$ of leg $A$ remains the same, while $\beta_{B}$ or $\mu_{B}$ for leg $B$ varies.

as gravitational acceleration is always the same. However, with the inclusion of the double-support phase, the gait dynamics will be different. The hip mass, $m_{H}$, and the mass of leg $A, m_{A}$, does not change. So referring to Equation (3.43); as the mass ratio $\mu_{B}$ increases, the magnitude of the mass of leg $B, m_{B}$, will decrease.

Figure 3-12 represents the effect of changing $\mu_{B}$ on various aspects of gait including inter-leg angle, step period, walking speed and energy lost due to collisions. From the analysis of gait characteristics, the stance time of a lighter leg was longer than for the unaltered leg (Figure 3-12b). These results oppose those from empirical data performed for amputees walking with prosthetic legs $[4,59,91,25]$. The phenomenon of a shorter stance time for the prosthetic limb may be due to a variety of reasons, such as a method to avoid pain and minimise discomfort on the amputated limb and to minimise the need for controlling the mechanical interaction between the prosthetic leg and the environment, as the healthy leg would have more control during stance support. Increasing $\mu_{B}$ by $30 \%$ increased stance time for leg $B$ by $2.45 \%$ and reduced stance time for the heavier leg $A$ by $2.15 \%$. These results suggest that the total mass of the limb has a direct effect of the stance time of the affected limb, however do not contribute to an amputees' shorter stance time. This model can also be used to estimate the collisional losses for redirecting the COM during the inelastic double- 
support collision. Figure 3-12d shows that although the mass of one leg, $m_{B}$, has an effect on the energy lost, the difference does not seem to be significant.

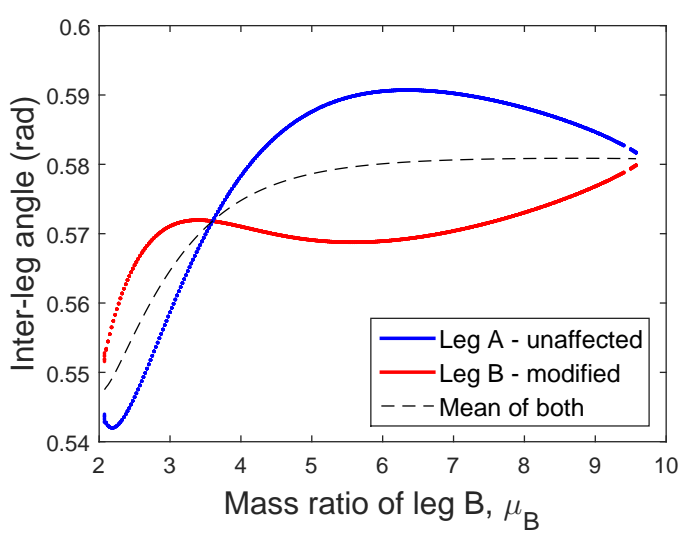

(a)

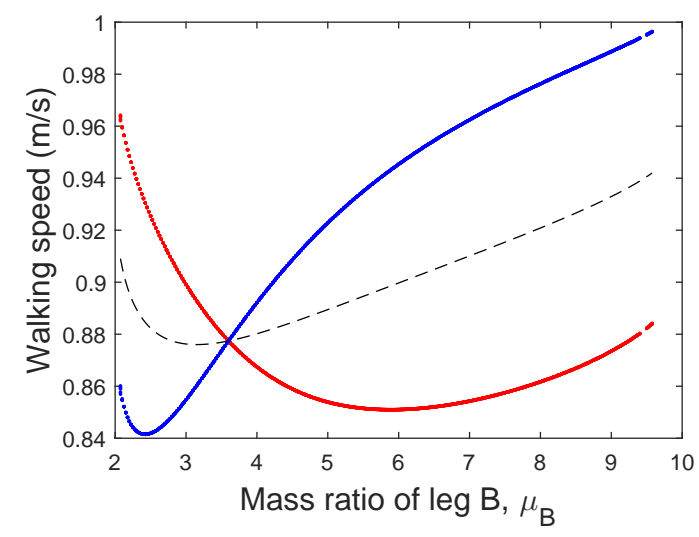

(c)

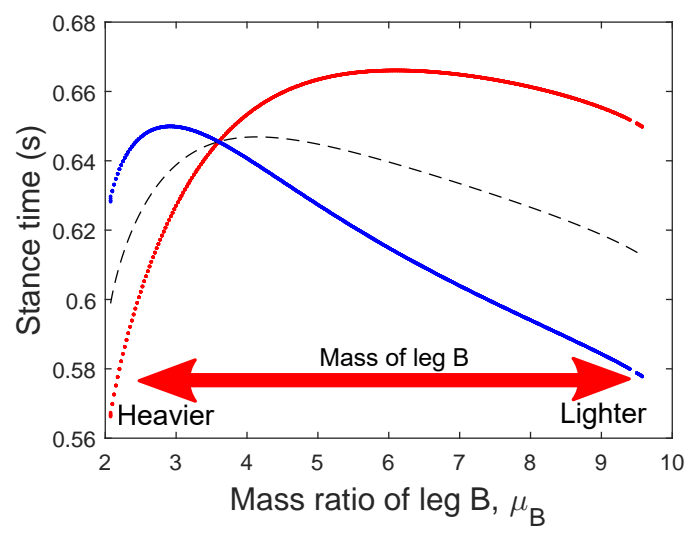

(b)

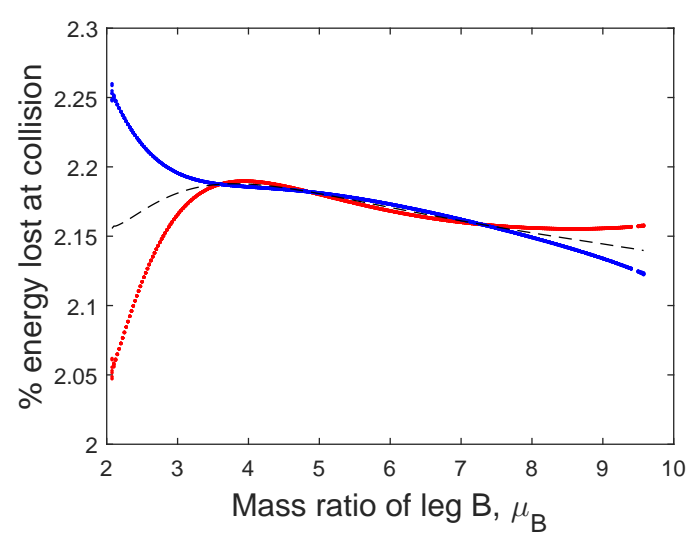

(d)

Figure 3-12: Effect of changing the leg mass ratio $\mu_{B}$ of one leg on the gait characteristics of the PW with a slope angle of $2^{\circ}$. The blue points represents gait characteristics while the unaffected leg is the support leg, while red points indicates that the modified leg is the support. The other leg remains at $\mu_{A}=3.6$

\section{Asymmetric leg mass position ratio, $\beta$}

In this case the effects of variation in length ratio (position of the leg COM) is investigated. As before the contralateral leg remains unchanged at a leg ratio, $\beta_{A}$, equal to 0.6. From Equation (3.45); as the ratio $\beta_{B}$ increases, the leg mass will be lower and further away from the hip mass, $m_{H}$. 
Bifurcation diagrams showing the difference in gait characteristics for both legs with respect to $\beta_{B}$ are shown in Figure 3-13. As $\beta_{B}$ increases, the average stance time increases (Figure 3-13b), while the walking speed decreases (Figure 3-13c). If $\beta_{B}$ is reduced by $35 \%$, the average stance time decreases by $10.66 \%$ while the average walking speed of both legs increases by $8.59 \%$. As $\beta_{B}$ increases there is also a larger step length during the higher massed leg in stance, while the leg with the lower mass has less of a change (Figure 3-13a). These results show that the average gait characteristics of both legs also change, for example increasing $\beta_{B}$ will decrease the stance speed of both legs as can be seen in Figure 3-13c. This means that symmetrising the difference between the two legs may not achieve optimal dynamics comparable to a healthy gait.

\subsubsection{Asymmetric roller radius, $R$}

As discussed in Section 2.1.6, the contact created by the human lower leg system conforms to a rocker that has a radius of approximately one third the leg length $[94,45,2]$. The model outlined in this chapter has the capability of using a polynomial function for the foot contact $[89,12]$, however the results in this section use a model with no defined foot length and radius of curvature, $R_{A}=0.3 L$, where $L$ is the length of the leg (and donates the position of hip mass, $m_{H}$ ). In this section the legs have a symmetric leg mass ratio and leg length ratio but a variation of rocker radius of a single leg, $R_{B}$.

Figure 3-14 represents the effect of $R_{B}$ on gait characteristics for each leg. By only changing the rocker radius of the one leg, the effect of asymmetric roll-over shapes can be observed. When viewing the inter-leg variation in Figure 3-14a, it is worth noting that due to geometric constraints, the inter-leg angle, $\theta_{2}$, is not exactly equal to $2 \theta_{1}$ because of the different rocker radii. These bifurcation plots have been the result of all stable solutions found within the range. For amputees, a shorter stance time with the unaffected leg has been attributed to pain in the stump or discomfort, however these 


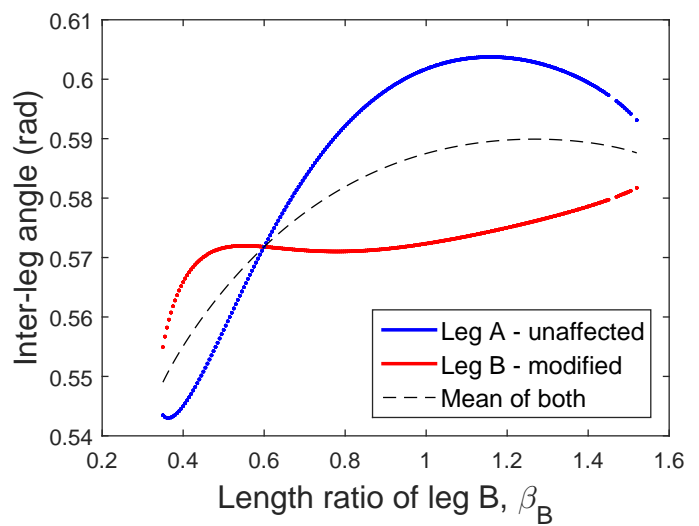

(a)

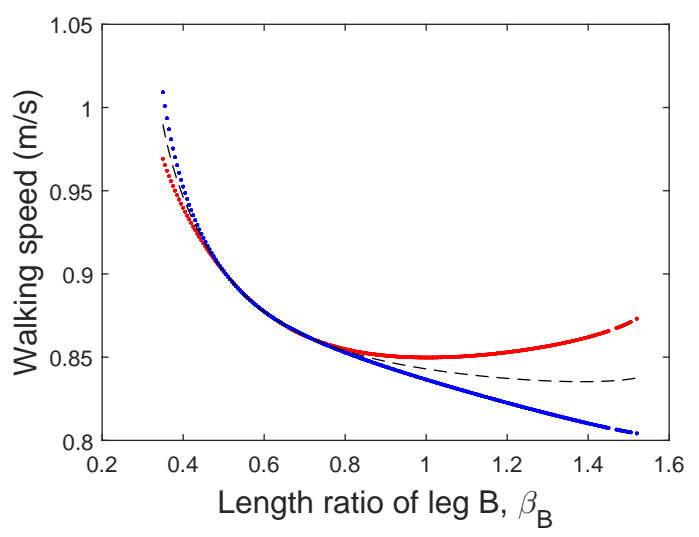

(c)

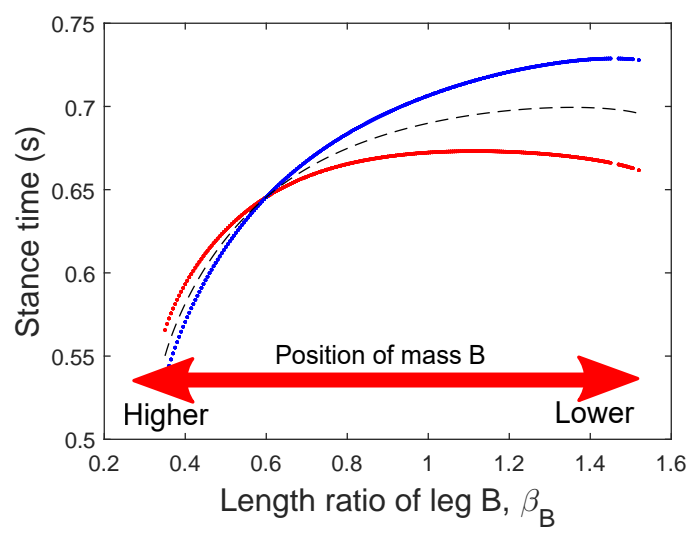

(b)

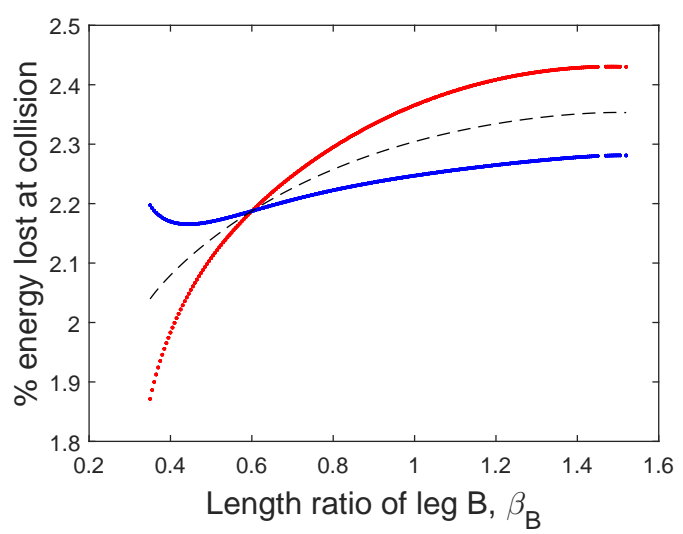

(d)

Figure 3-13: Effect of changing the leg length ratio $\beta_{B}$ of one leg on the gait characteristics of the PW with a slope angle of $2^{\circ}$. The blue points represents gait characteristics while the unaffected leg is the support leg, while red points indicates that the modified leg is the support. The other leg remains at $\beta_{A}=0.6$

results show that this may be due to the inability of some prosthetic feet to match an appropriate roll-over shape. There is an almost symmetrical relationship between the difference in rocker radii on most of the gait characteristics, whether it increases or decreases; the asymmetric nature is more apparent rather than the average effect on gait parameters. Figure $3-14 \mathrm{~d}$ represents the percentage of mechanical energy loss due to the inelastic collision at double-support. Although there is a significant difference between the two legs, the average energy lost over a range of steps in not significant. 


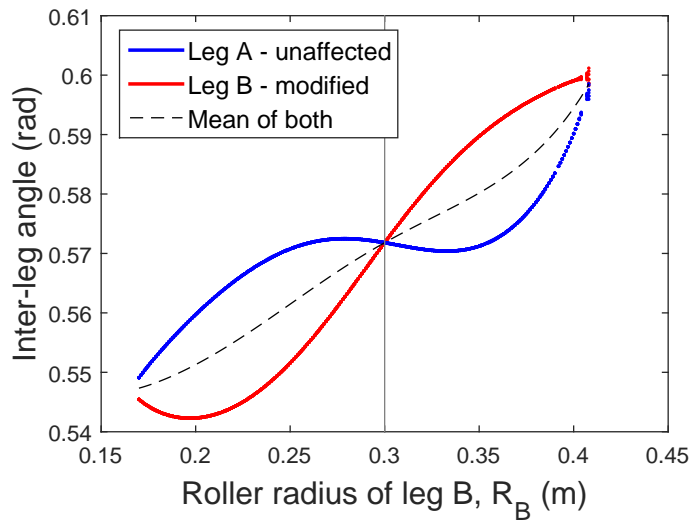

(a)

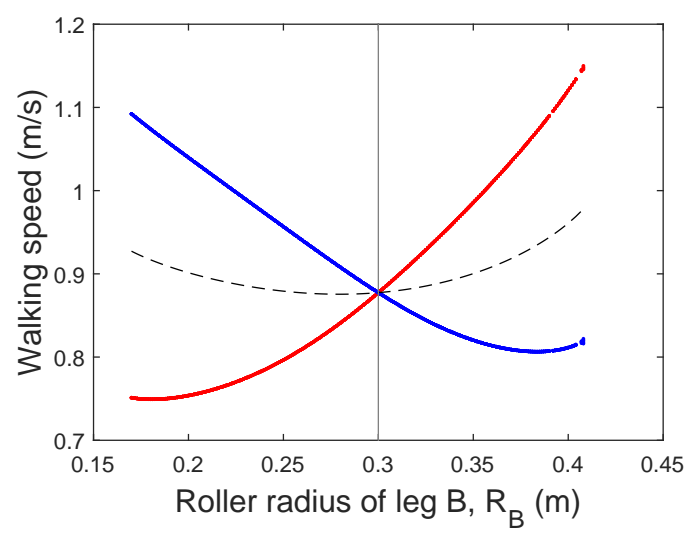

(c)

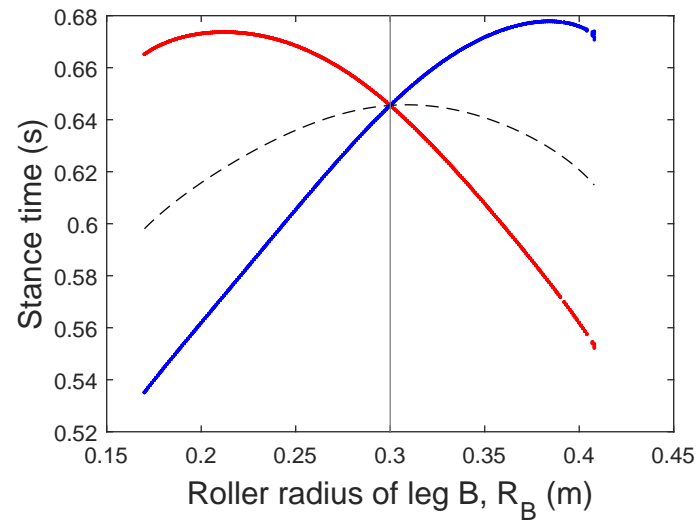

(b)

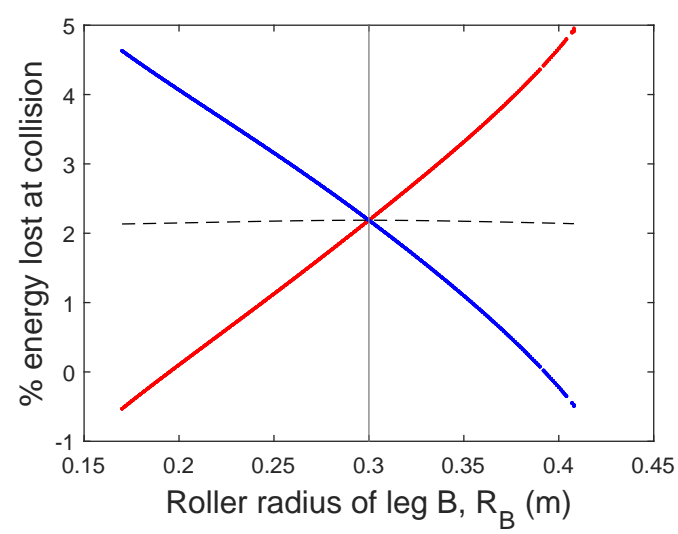

(d)

Figure 3-14: Effect of changing the radius of curvature $R_{B}$ of one leg on the gait characteristics of the PW with a slope angle of $2^{\circ}$. The blue points represents gait characteristics while the unaffected leg is the support leg, while red points indicates that the modified leg is the support. The other leg remains at $R_{A}=30 \%$ leg length.

\subsection{Discussion of results}

This chapter has used a passive walking model with curved feet and independent leg parameters to research asymmetric walking characteristics. It is worth noting that these results represent the behaviour expected if neurological controllers are not involved - as the passive walking model has no active controllers. As has been discussed in Section 2.3.2, the human system adapts to changes in inertial characteristics; these results informs us how the dynamics are affected due to mass perturbation and asymmetric roll-over shapes. 
An amputee has a shorter stance time on their prosthetic foot $[4,59,91,25]$. This is normally attributed to reasons such as pain or discomfort in the residual limb, reduced confidence in prosthetic support etc. These results suggest that, although the inertial characteristics of the limb have an effect on stance time (Figures 3-12b \& 3-13b), a lighter leg is predicted to have a longer stance time. A prosthetic leg's higher $\mu$ and lower $\beta$, lead to dynamics that inherently alter stance time of the lighter prosthetic foot. Both the length ratio and mass ratio of a prosthetic limb or other device should be considered as both affect the stance time, step length and associated asymmetry. For example, a heavy bionic foot would induce a shorter stance time on the prosthetic. These models can also be used to predict the ratio of mechanical energy lost due to the double-support redirection of the COM. Figures 3-12d and 3-13d represent the variation of collisional losses, although they do not appear to be significant.

The effect of roll-over shape also has an effect on the symmetry of gait. The radius of curvature of the roll-over shape in human walking does not change with respect to walking speed [47], shoe heel height [45], while carrying extra weight [46] and walking on inclined surfaces [48]. However if a person's gait is impaired, this may have an effect of the roll-over shape an individual is able to conform to. The effect of asymmetric roll-over shapes has been explored, noting that this may lead to different stance times, walking speeds and collisional energy for each step (Figure 3-14). It is also important to note that changing the parameters of one leg has an effect on the gait characteristics of both the altered and unaltered legs.

As new research is done on prosthetics, orthotics and exoskeletons: these results are important for understanding the difference in gait dynamics from the effect of asymmetric loading of leg masses. The different inertias of the legs lead to a change in gait characteristics and lead to a user having different strategy in leg kinetics to match kinematics [122]. The next step is to add a spring to the passive walking model to match the correct GRF pattern. The effect of effective stiffness of an affected leg will also be investigated. 


\section{Chapter 4}

\section{Asymmetric spring loaded inverted pendulum}

In the previous chapter, a passive walking model was used to study asymmetric gait dynamics. Compass walkers exhibit human-like gait behaviour, however they do not model the same M-shaped ground reaction force (GRF) pattern as a normal person. Passive walkers also have an instantaneous double-support phase, which is not comparable to human walking, as the double-support of human walking lasts approximately $20-27 \%$ of a stride [1].

Traditionally, the inverted pendulum is used to predict the centre of mass (COM) trajectory during walking, while a springy/compliant leg gave good predictions for running models. Geyer et al. [39] adapted the spring loaded inverted pendulum (SLIP) running model into a model to represent walking gait. This induced GRFs comparable to human gait and marked an improvement over the inverted pendulum to correctly match the human gait kinematics. This also follows the philosophy in this thesis that human-like characteristics can be obtained from passive models with no active controllers. In previous research, comparisons have been made between the spring-mass model and human walking data [81] and it has been found that 
parameters such as effective leg stiffness and touch-down angle may be estimated directly from experimentally observed data; force-length curves and motion data. Although the SLIP model can give good prediction for moderate walking (and medium running) speeds, the model does not provide successful simulations at faster walking speeds. Whittington and Thelen [147] extended the SLIP model by adding roller feet and found that to match ground reactions measured during normal human walking, simultaneous modulation of limb touch-down angle and limb stiffness were needed to induce speed-related changes.

Previous models simulate locomotion with equal parameters for both legs. In this chapter the spring-mass walking model is extended to incorporate asymmetry. Asymmetric touch-down angle and effective stiffnesses for each leg will be investigated in Section 4.3 to see their effect on the gait dynamics. As with the previous chapter, asymmetric roll-over shapes will also be investigated. However as the SLIP model only has a hip mass with no leg masses, asymmetric leg inertias cannot be investigated using this model. Gait characteristics such as step length, stance time and maximum GRF peaks will be described and comparisons made to human gait.

\subsection{Equations of motion for the spring loaded in- verted pendulum}

In this section the equations of motion are derived for a system similar to the SLIP model with roller feet derived by Whittington and Thelen [147], however the state of the model is represented by the stance leg angle, $\theta$ and the leg extension, $r$, as the degrees of freedom instead of the $[X, Y] \mathrm{COM}$ coordinates. In comparison to the previous model in Chapter 3, the mechanical energy of the system remains constant as there are no collisional losses and the ground is level (slope angle $\gamma=0$ ).

Figure 4-1 represents the schematic of the SLIP model. Let the location of mass 


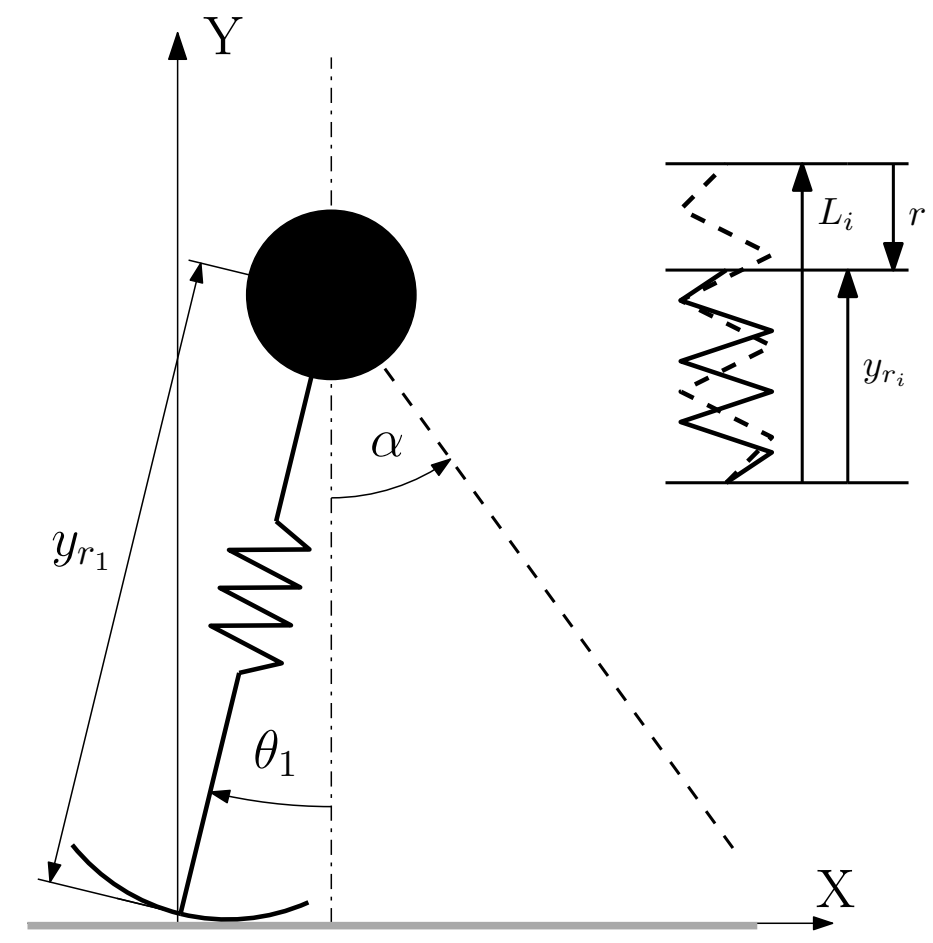

Figure 4-1: Spring loaded inverted pendulum (SLIP) model with roller foot during single-support.

be represented by

$$
\begin{aligned}
& X=-x_{\theta}(\theta) \cos (\theta)+\left(y_{r}(r)-y_{\theta}(\theta)\right) \sin (\theta)+s(\theta) \\
& Y=x_{\theta}(\theta) \sin (\theta)+\left(y_{r}(r)-y_{\theta}(\theta)\right) \cos (\theta)
\end{aligned}
$$

Where $y_{r}$ is the leg length and $y_{r}=L_{0}+r ; L_{0}$ being the original leg length. $x_{\theta}$ and $y_{\theta}$ represent the local coordinates of the COP along the foot as in Section 3.1.1. The model's potential energy is a combination of gravitational potential and spring potential,

$$
V=m g Y+\frac{1}{2} m k r^{2}
$$

and the kinetic energy is a function of the hip mass velocity vector,

$$
K=\frac{1}{2} m\left|\overrightarrow{\boldsymbol{v}}_{1}\right|^{2}=\frac{1}{2} m\left(\dot{X}^{2}+\dot{Y}^{2}\right)=\frac{1}{2} m\left(\left(\frac{\partial X}{\partial \theta} \dot{\theta}+\frac{\partial X}{\partial r} \dot{r}\right)^{2}+\left(\frac{\partial Y}{\partial \theta} \dot{\theta}+\frac{\partial Y}{\partial r} \dot{r}\right)^{2}\right)
$$

The total Mechanical Energy (ME) is the sum of kinetic energy, $K$, and potential 
energy, $V$ :

$$
\mathrm{ME}=\frac{1}{2} m\left(\left(\frac{\partial X}{\partial \theta} \dot{\theta}+\frac{\partial X}{\partial r} \dot{r}\right)^{2}+\left(\frac{\partial Y}{\partial \theta} \dot{\theta}+\frac{\partial Y}{\partial r} \dot{r}\right)^{2}\right)+m g Y+\frac{1}{2} m k r^{2}
$$

The equations of motion are derived via the Lagrange equation

$$
\frac{d}{d t}\left(\frac{\partial \mathcal{L}(\boldsymbol{q}, \dot{\boldsymbol{q}})}{\partial \dot{\boldsymbol{q}}}\right)-\frac{\partial \mathcal{L}(\boldsymbol{q}, \dot{\boldsymbol{q}})}{\partial \boldsymbol{q}}=0
$$

and to maintain consistent notation with Chapters $3 \& 5$, the equations of motions may be written as:

$$
\boldsymbol{M}(\boldsymbol{q}) \ddot{\boldsymbol{q}}+\boldsymbol{N}(\boldsymbol{q}, \dot{\boldsymbol{q}}) \dot{\boldsymbol{q}}+\boldsymbol{V}^{\prime}(\boldsymbol{q})=0
$$

with $\boldsymbol{q}=[\theta, r]$ and the matrices given as

$$
\begin{gathered}
\boldsymbol{M}(\boldsymbol{q})=\left[\begin{array}{ll}
M_{11} & M_{12} \\
M_{21} & M_{22}
\end{array}\right] \\
\boldsymbol{N}(\boldsymbol{q}, \dot{\boldsymbol{q}})=\left[\begin{array}{ll}
N_{11} & N_{12} \\
N_{21} & N_{22}
\end{array}\right] \\
\boldsymbol{V}^{\prime}(\boldsymbol{q})=\left[\begin{array}{ll}
\frac{\partial V}{\partial \theta} & \frac{\partial V}{\partial r}
\end{array}\right]^{T}
\end{gathered}
$$

with $V$ being the potential energy of the system (Equation 4.2) and the values of matrices in Equations (4.7) \& (4.8) being

$$
\begin{gathered}
M_{i j}=m\left(\frac{\partial X}{\partial q_{i}} \frac{\partial X}{\partial q_{j}}+\frac{\partial Y}{\partial q_{i}} \frac{\partial Y}{\partial q_{j}}\right) \\
N_{i j}=\sum_{n=1}^{2} \frac{\partial M_{i n}}{\partial q_{j}} \dot{q}_{n}-\frac{1}{2} \sum_{n=1}^{2} \frac{\partial M_{j n}}{\partial q_{i}} \dot{q}_{n}
\end{gathered}
$$

where $i$ and $j$ represent the location in the matrix and $n$ is the number of variables (which is equal to two $-\theta$ and $r$ ). 


\subsubsection{Double-support phase}

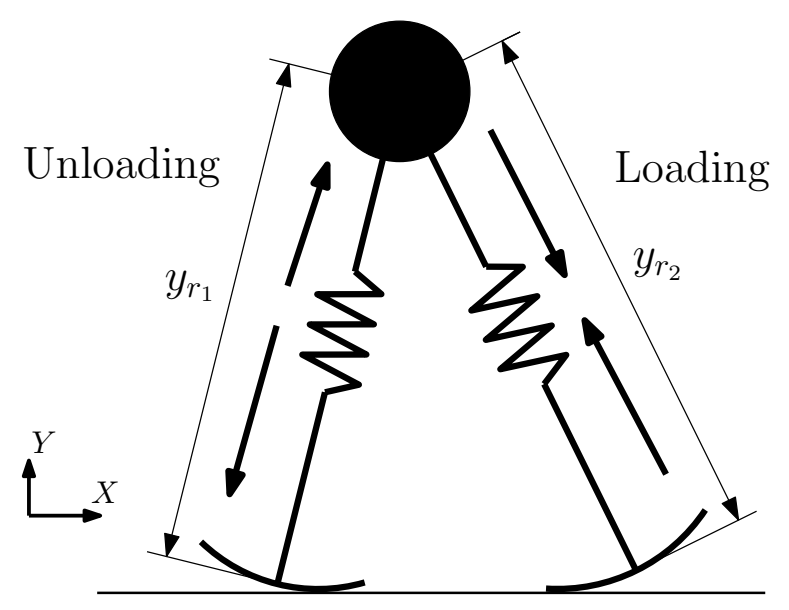

Figure 4-2: SLIP model with roller foot during double-support. The front leg's load increases, as the rear leg extends before take-off.

During single-support the extension of the swing leg $r_{2}$ is not modelled so is equal to 0 when calculating the energy in Equation (4.4). Instead the swing leg is assumed to land at a defined touch-down angle $\alpha$. Once the front leg spring makes contact with the ground, the touch-down event occurs. At the double-support there is no slipping so the front leg is constrained by the touch down point. The extension of the front leg at double-support is represented as $r_{2}\left(\theta, r_{1}\right)$. Therefore at any stage the walker may be represented by stance angle and spring length, $\boldsymbol{q}(t)=\left[\theta(t), r_{1}(t)\right]$. With both springs in contact with the floor, the potential energy becomes

$$
V=m g Y+\frac{1}{2} m k_{1} r_{1}^{2}+\frac{1}{2} m k_{2} r_{2}^{2}
$$

and the total mechanical energy

$$
\mathrm{ME}=\frac{1}{2} m\left(\left(\frac{\partial X}{\partial \theta} \dot{\theta}+\frac{\partial X}{\partial r} \dot{r}\right)^{2}+\left(\frac{\partial Y}{\partial \theta} \dot{\theta}+\frac{\partial Y}{\partial r} \dot{r}\right)^{2}\right)+m g Y+\frac{1}{2} m k_{1} r_{1}^{2}+\frac{1}{2} m k_{2} r_{2}^{2}
$$

A schematic of the model in double-support is shown in Figure 4-2. After the front leg makes contact with the ground, the front spring $r_{2}$ compresses and the rear leg spring $r_{1}$ extends until $r_{1}$ is equal to 0 . At this point, rear leg contact is lost and 
the model switches to the single-support phase again with the front leg being the new support leg.

\subsubsection{Numerical implementation}

Similar to the previous model as explained in Section 3.1.4, the walker is given a set of random initial conditions until stability is reached or there is no successful solution after 500 attempts. A full walking step comprises of hybrid dynamics. The singlesupport phase ends either if the walker falls over or the touch-down event occurs; when the front swinging leg makes contact with the ground. If the touch-down event occurs, the double-support dynamics are initiated and this ends when the rear leg loses contact with the floor (when $r=0$ the leg is fully extended). Once this situation is reached, the next step initiates and the model transfers to single-support with the opposite leg.

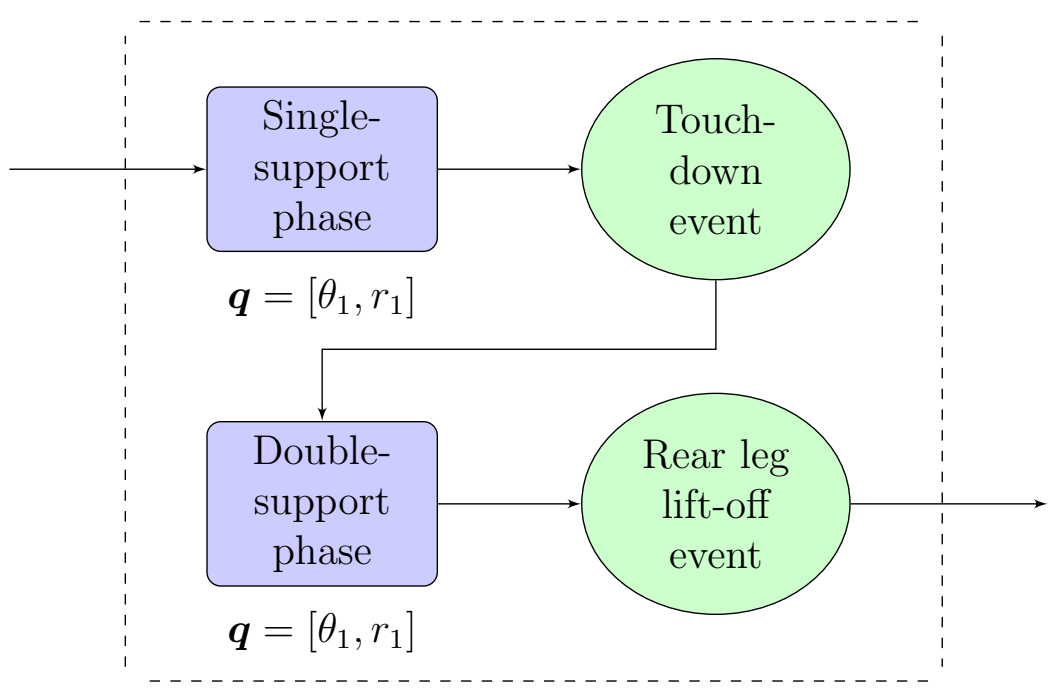

Figure 4-3: Flowchart of a complete walking step in the SLIP model. Can be integrated as the 'Complete Walking Step' block in Figure 3-5. 


\subsubsection{Validation of results}

Figure 4-4 demonstrates the similarities between the SLIP model derived in this chapter and the model derived from Whittington and Thelen [147]. The main differences between the two systems is that Whittington and Thelen's model has walking speed as an input with the initial conditions altered to match the objective walking speed and symmetry is maintained as $\dot{r}=0$ at mid-stance. Whereas the current model uses ME as a parameter input and relies on eventual stable walking, with symmetry verified with bifurcation diagrams. Regardless of this, there is an average difference of $1.95 \%$ in the values between the maximum GRF of each roller radius in Figure 4-4. The advantage of this SLIP is that asymmetry is possible and a constant metabolic energy cost can be assumed. This provides useful insights when investigating asymmetry as in Section 4.3.

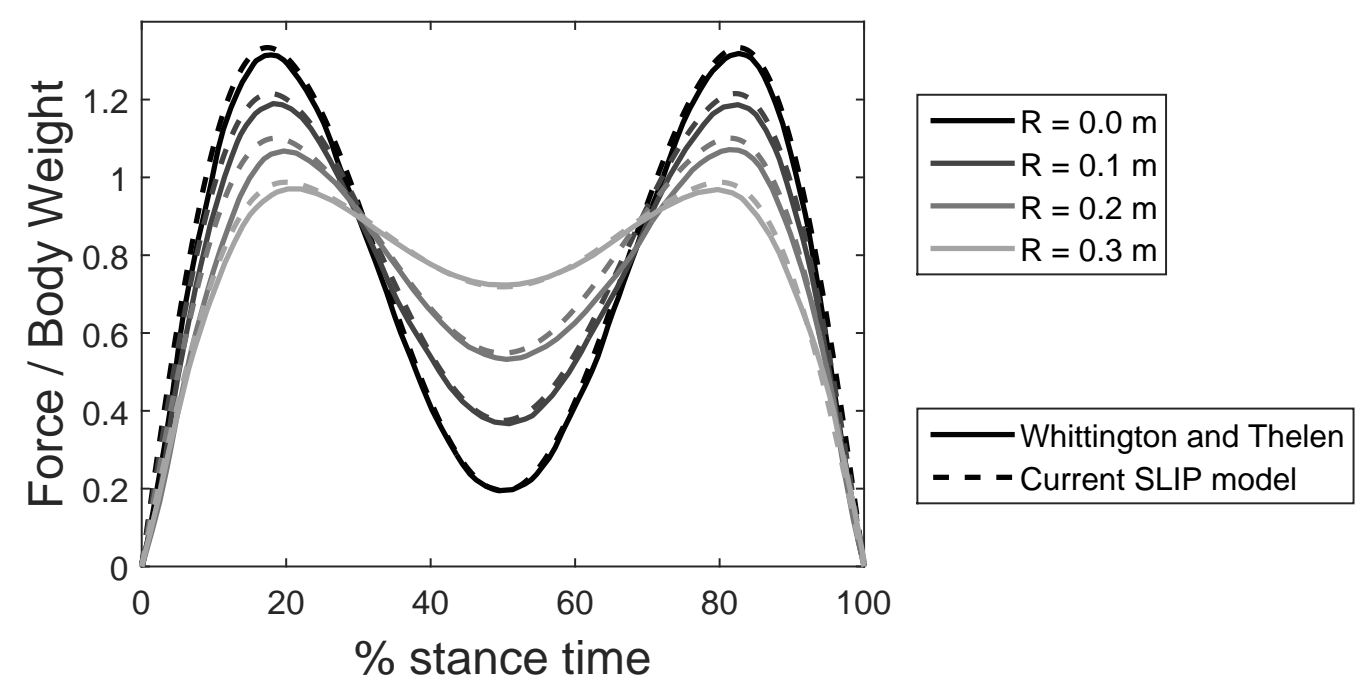

Figure 4-4: Comparison between the current SLIP model results and the results obtained from Whittington and Thelen [147]. Model parameters are $k=20 \mathrm{kN} / \mathrm{m}$, $\alpha=20^{\circ}, m=80 \mathrm{~kg}, L=1 \mathrm{~m}$ and a target walking speed of $1.35 \mathrm{~m} / \mathrm{s}$ 


\subsection{Evaluating model results}

\subsubsection{Limit cycle of stable walking solution}

Similar to the previous model in Chapter 3, if given a set of stable initial conditions for $[\boldsymbol{q}, \dot{\boldsymbol{q}}]=[\theta, r, \dot{\theta}, \dot{r}]$, the model will settle to a periodic gait cycle. The sequence graph shown in Figure 4-5a shows that the gait will settle to a steady cycle after a number of walking steps. It is worth noting that although it may seem that this system is experiencing dissipation, there is no energy loss in this model. Ruina [112] has noted previously that a conservative system may demonstrate an apparent asymptotic stability. Figure 4-5b represents the cyclic pattern of the vertical trajectory of the COM during a steady, stable gait pattern. If the dynamics are compared to the passive-walking model (Section 3.2.2), the SLIP model seems to oscillate a lot more, taking a greater number of walking steps before settling to a steady gait.

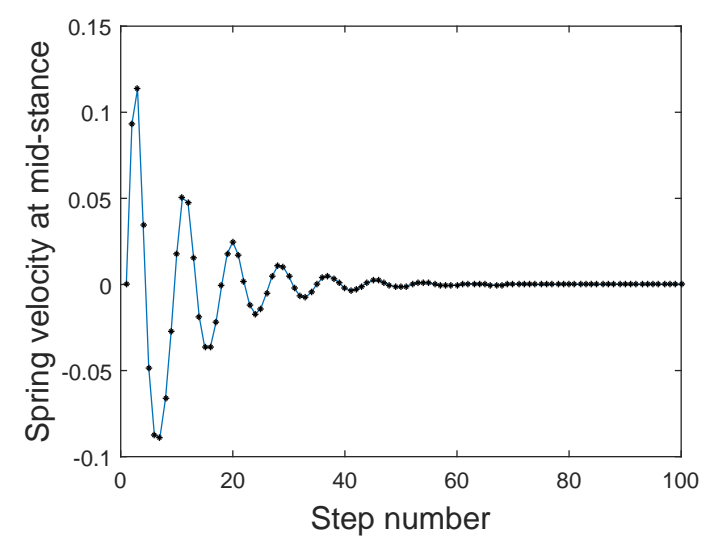

(a)

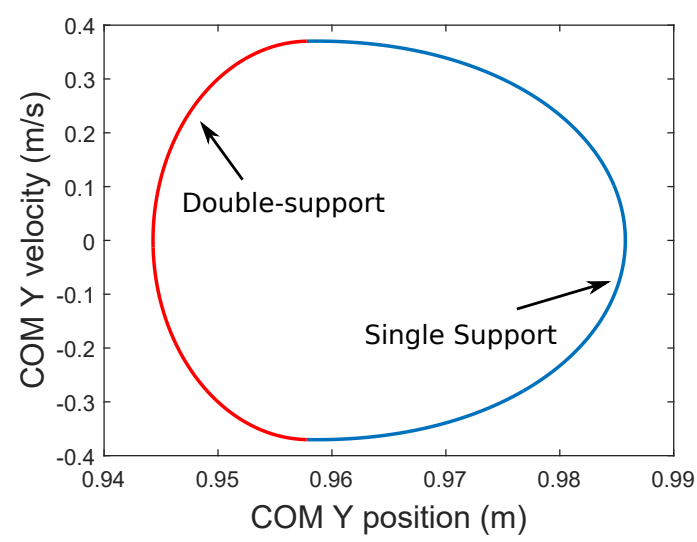

(b)

Figure 4-5: (a) Stable sequence walking up to 100 steps shows that the model converges to a steady gait from a random set of initial conditions. (b) Vertical COM trajectory for each step exhibits a repeated cyclic pattern. 


\subsubsection{Ground reaction forces}

The Ground Reaction Forces (GRFs) provide insight into how the COM is propelled/supported by each individual limb. A standard vGRF pattern is shown in Figure 4-6. In normal human walking, the vertical GRF curve is M-shaped with two distinct peaks; FY1 is the value of the first peak and FY2 is the second. The horizontal GRF provides insight into the braking and propulsion performed by the limbs.
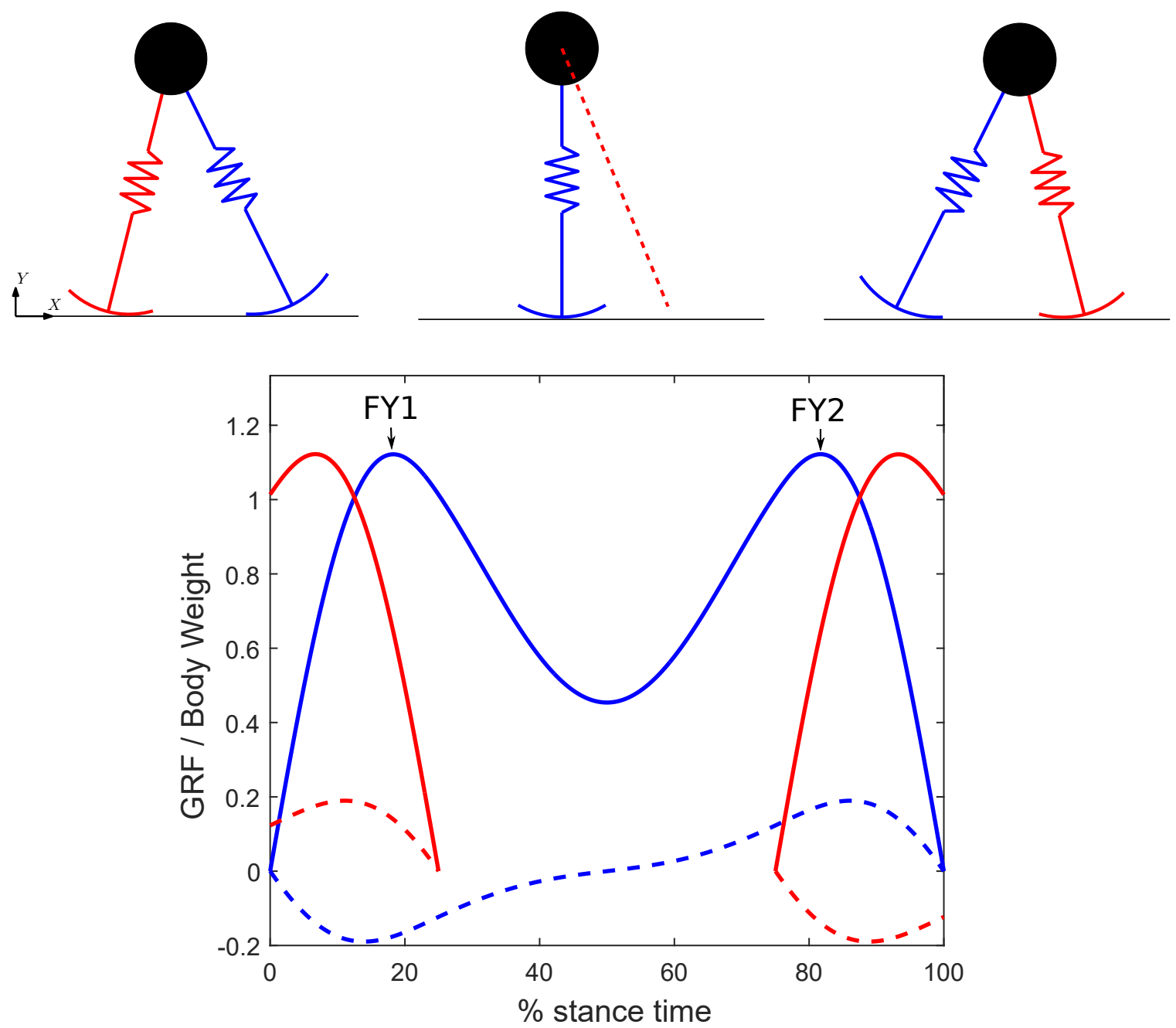

Figure 4-6: Ground reactions forces in the $\mathrm{X}$ (dotted) and $\mathrm{Y}$ (solid) planes of the leg during stance phase (blue). The GRFs of the other leg is shown during doublesupport (red).

The horizontal impulse of each step is also calculated. Integrating the horizontal 
GRFs as in Figure 4-7 can be used to quantify the contribution from each limb to the braking or propulsion of the body mass. The net impulse represents the momentum change from each limb:

$$
\int_{0}^{T} F_{x} \mathrm{~d} t=m \Delta v_{x}
$$

so the net horizontal impulse, $m \Delta v_{x}$, is obtained by integrating the total horizontal GRF, $F_{x}$, over the total stance time the limb is in contact with the ground, $T$.

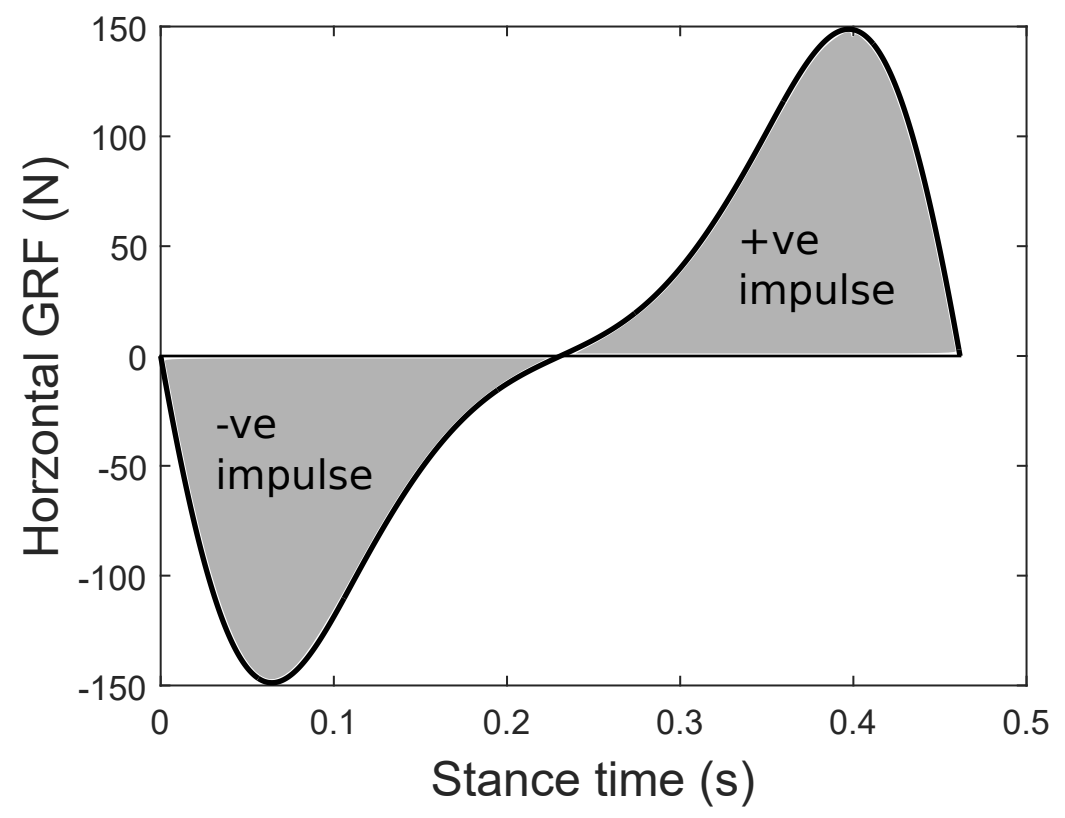

Figure 4-7: The net impulse contribution from each leg is calculated by integrating the horizontal Ground Reaction Forces (GRFs) during the total stance phase.

\subsubsection{Walking parameter domains}

Model parameters such as leg length and body mass can be set to an appropriate value arbitrarily close to the average human parameters. Other parameters such as Mechanical Energy (ME), touch-down angle, $\alpha$ and effective leg stiffness, $k$, can be estimated from experiments but are more difficult to define in this study as a person may adapt these values based on walking speed [81, 147].

Figure 4-8 shows stable walking is found at certain combinations of ME, $\alpha$ and $k$ 
with initial spring compression at mid-stance, $r_{0}$ of $15 \mathrm{~mm}$ and spring velocity, $\dot{r}_{0}$ of 0. If the model reaches a stable walking pattern, a voxel is plotted. Empty squares mean that no stable solution was found from the set of initial conditions. Domains are separated by the number of 'humps' of GRF plot with different modes of locomotion found; the model having stable solutions with two to six GRF peaks found within the parameter space shown. In this thesis, interest is directed to the double-hump GRF pattern as this is the common pattern seen is human walking [62]. The number of peaks in the GRF pattern are checked from the MATLAB peaks function and verified via inspection.

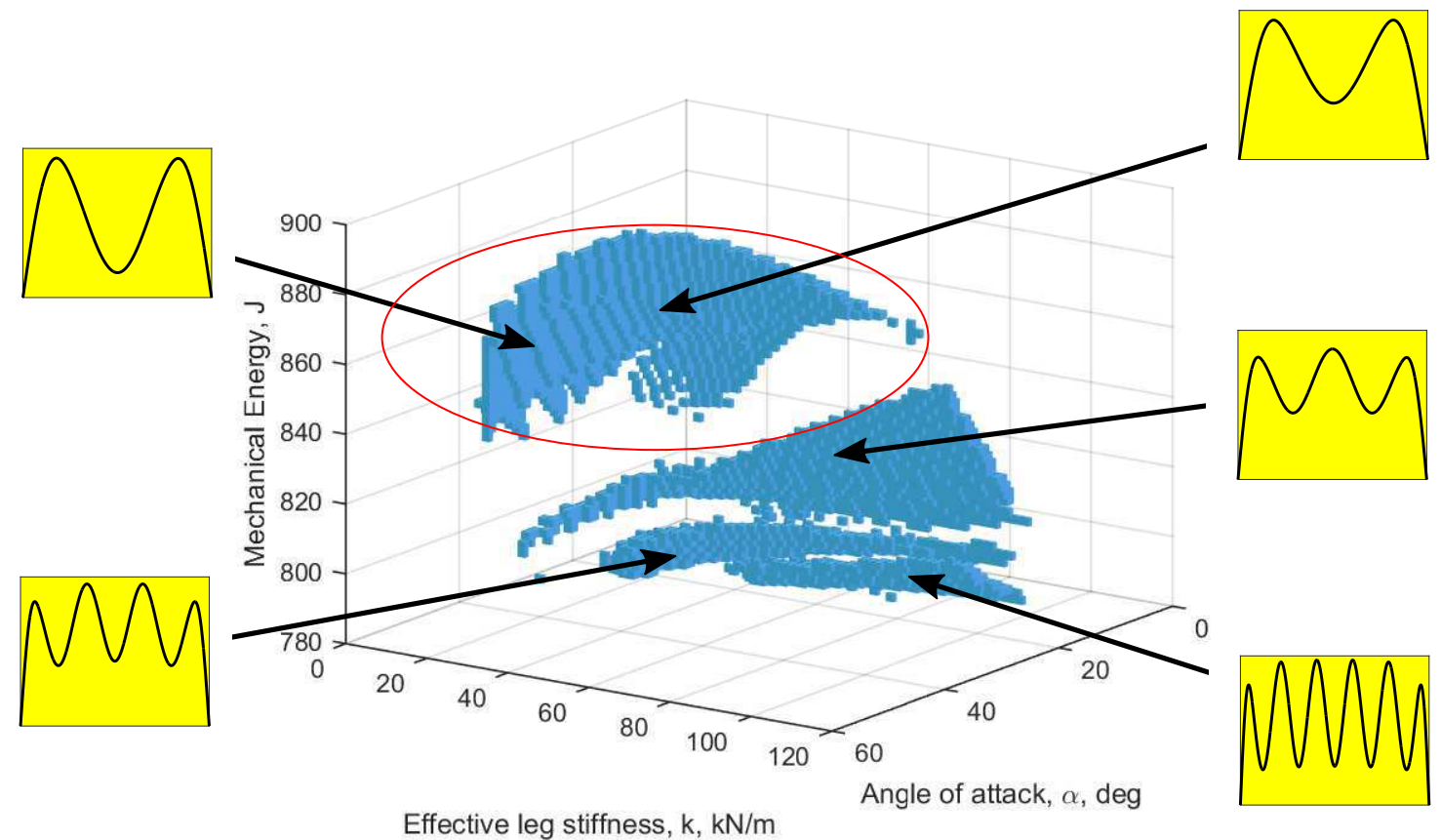

Figure 4-8: Parameter domain showing stable walking conditions at combinations of touch-down angle, effective leg stiffness and mechanical energy. Outside plots represent the vertical GRF pattern exhibiting two to six peaks seen in this parameter domain. Only two-peak vGRF patterns (region within red ellipse) are considered in this chapter. 


\subsubsection{Gait characteristics}

To help gain understanding of this model, gait characteristics are evaluated at stable walking conditions. These offer events comparable to human experiments so that corresponding observation may be made. The gait characteristics later used in Figures 4-9, 4-11 \& 4-13 are: (a) inter-leg angle between the legs, $\theta_{2}$, at the end of the step at touch-down; (b) stance time, the total time the limb is in contact with the ground for each step (i.e. From heel-strike to toe-off); (c) walking speed, distance per unit time from touch-down to opposite touch-down and (d) net horizontal impulse during the stance phase from Equation 4.14. The vertical Ground Reaction Force (vGRF) has also been quantified by the maximum (e) first vGRF peak, FY1, and (f) second vGRF peak, FY2, observed for each leg.

Gait characteristics will be evaluated to see the effect of changing one leg's touchdown angle or leg stiffness to the symmetry of gait. Schaarschmidt et al. [118] notes that amputees alter their touch-down angle of the prosthetic to enable forward propulsion on the prosthetic, while load bearing is shifted to the intact leg. These springmass walking models also confirm that a larger $\alpha$ increases the peak FY1, while a shallower $\alpha$ increases FY2 [113]. A faster walking speed also increases both of these GRF peaks.

\subsubsection{Standard model parameters used}

Parameters are selected so that the model's specification is close to human anthropology to better compare walking data as previously in Section 3.2.1. Table 4.1 outlines the variable used for each parameter, unless specifically altered in each section. As the effects of asymmetry are to be investigated in this chapter, these standard parameters are selected to provide moderate walking speeds; as this have been shown to provide good predictions for the SLIP model [81]. 


\begin{tabular}{lcc}
\hline Name & Notation & Value \\
\hline Natural leg length & $L$ & $1 \mathrm{~m}$ \\
Total mass & $\sum m$ & $80 \mathrm{~kg}$ \\
Radius of gyration/leg length & $\mathrm{R}$ & 0.3 \\
Touch-down angle & $\alpha$ & $20^{\circ}$ \\
Effective leg stiffness & $k$ & $25 \mathrm{kN} / \mathrm{m}$ \\
Mechanical Energy & $\mathrm{ME}$ & $860 \mathrm{~J}$ \\
\hline
\end{tabular}

Table 4.1: Standard parameters used for the SLIP model.

\subsection{Investigating asymmetry in the spring-loaded inverted pendulum}

Walking is assumed to be symmetric, however injury or neuromuscular diseases may effect the symmetry of a person's gait. In this section the effect of asymmetric touchdown angle $\alpha$ and effective leg spring stiffness $k$ will be investigated. The effects of an asymmetric roll-over shape will also be explored by investigating asymemtric roller radius, $R$. The consequence of these asymmetries on the gait characteristics for both legs are evaluated. These results will also be used to see if altering leg touch-down angle or effective leg stiffness is a possible mechanism for adapting gait dynamics. Experiments can only evaluate differences between an intact and an altered leg, it can be hard to compare to a normal gait. Using this model, the dynamic effects will be explored to gain understanding of asymmetric gait dynamics without any adaptive controllers.

\subsubsection{Asymmetric touch-down angle, $\alpha$}

The leg touch-down angle may be used as a control mechanism for desired gait mechanics [143]. In this model the legs are massless, with the touch-down angle, $\alpha$, as a constant to represent the angle of the swing leg at the point of heel-strike. Simulations of the model are performed with the model having arbitrary parameters as described in Table 4.1. One leg has a constant touch-down angle, $\alpha_{A}$ of $20^{\circ}$, while 
variation of the other leg's touch-down angle, $\alpha_{B}$, is observed. Figure 4-9 represents the stable gait characteristics as a function of $\alpha_{B}$

Inter-leg angle is a function of touch-down angle, $\alpha$, and stance leg angle, $\theta$, for a given spring compression, $r_{1}$, at heel-strike. It seems natural that increasing $\alpha$ would increase the step length for the contralateral leg (Figure 4-9a). Interestingly, although the stance time increases with $\alpha_{B}$, this gait characteristic remains symmetric although the model has asymmetric touch-down angles between each leg (as can be seen in Figure 4-9b). Asymmetry is seen in the walking speed for each leg (Figure 4-9c), with a larger touch-down angle decreasing the speed of the next step. If the touch-down angle of leg $B, \alpha_{B}$, is $5^{\circ}$ shallower, the smaller step length of leg $B$ increases stance speed by $17 \%$, while the speed during stance of the contralateral leg $A$ decreases by $28 \%$. An altered touch-down angle modifies the step length and walking speed while maintaining symmetric stance-time for both legs.

Figure 4-9d represents the net horizontal impulse provided by each leg during stance phase. The leg with a larger touch-down angle contributes to slow down the body mass velocity, while the propulsion from the other leg increases. As the touchdown angle increases, although the vertical GRF peaks become more asymmetric, the maximum value of the first peak value FY1 does not decrease significantly. If the stance phase is initiated with a larger touch-down angle, the COM is propelled with a larger force than the contralateral leg. Figures 4-10a and 4-10b show the vertical GRFs with $\alpha_{B}$ at $15^{\circ}$ and $23^{\circ}$ respectively. As the touch-down angle becomes shallower, the trough at mid-stance becomes lower and the model approaches take-off failure as shown in Figure 4-10a. If the spring retracts completely before the other leg makes contact with the ground, the model takes off during stance phase which is characterised by a running gait. Empirical evidence suggest that amputees increase their intact leg angle of the prosthetic foot [60,92] which has the effect of minimising GRFs while increasing propulsion [113]. 


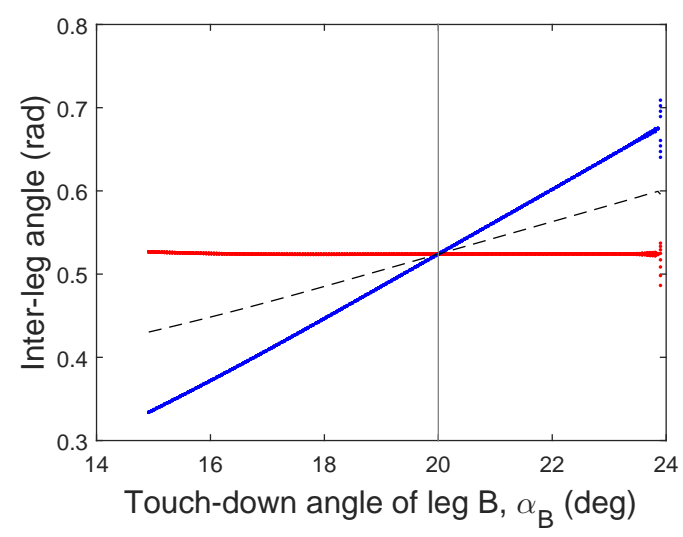

(a)

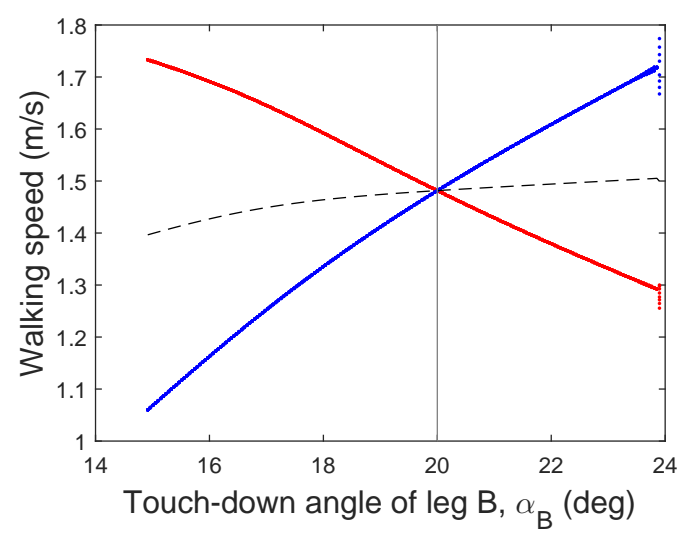

(c)

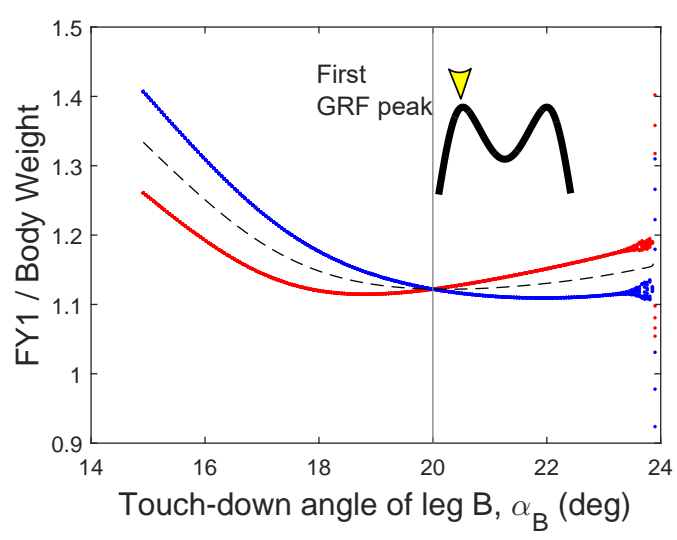

(e)

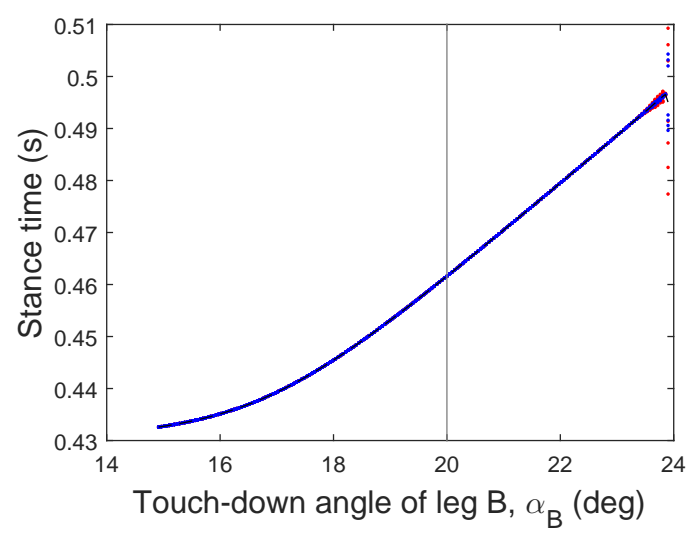

(b)

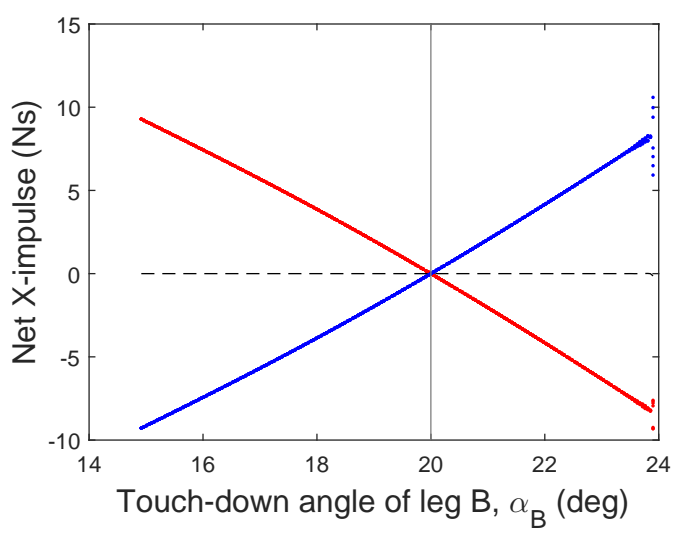

(d)

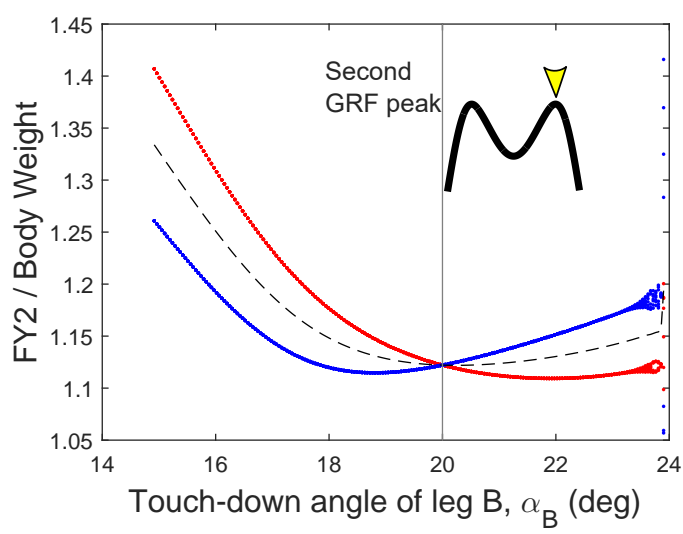

(f)

Figure 4-9: Effect of changing the touch-down angle of one leg, $\alpha_{B}$, on the gait characteristics of the SLIP model. The blue points represents gait characteristics while the unaffected leg is the support leg, the red points indicates that the modified leg is the support. The other leg remains at $\alpha_{A}=20^{\circ}$. 


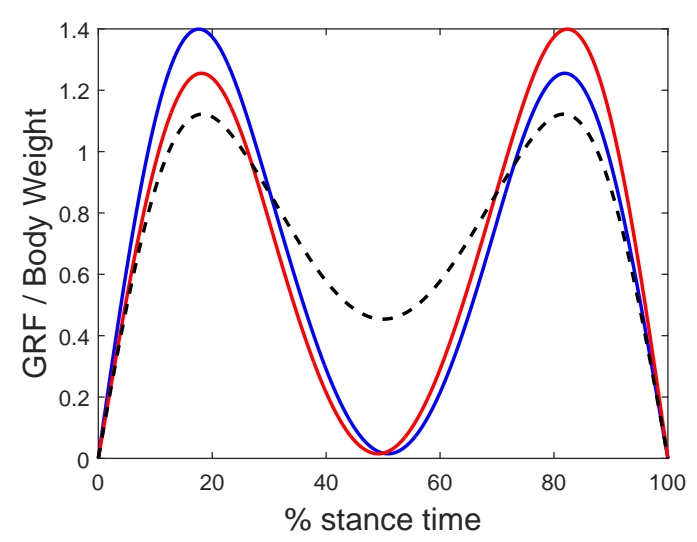

(a) $\alpha_{B}=15^{\circ}$

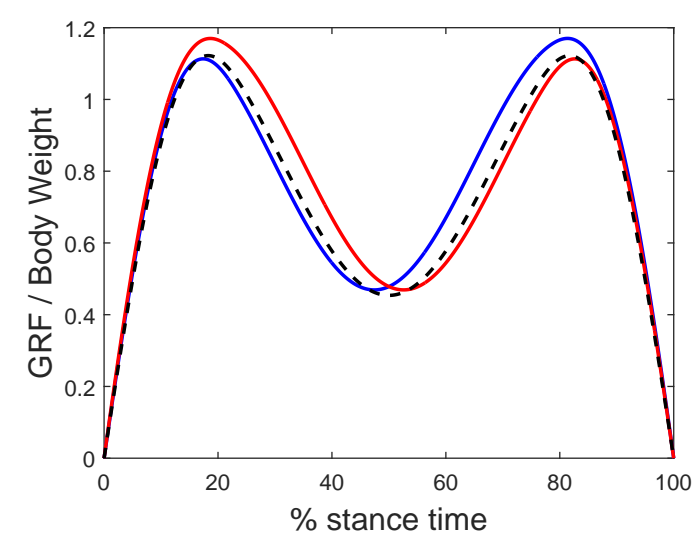

(b) $\alpha_{B}=23^{\circ}$

Figure 4-10: Vertical Ground Reaction Force (GRF) profile for the SLIP walking model with the touch-down angle of $\operatorname{leg} B, \alpha_{B}$, being equal to (a) $15^{\circ}$ and (b) $23^{\circ}$ (shown by the red line), while the other leg touch-down angle, $\alpha_{A}$, remains at $20^{\circ}$ (shown by the blue line). The GRF plot for a symmetric model with $\alpha$ being $20^{\circ}$ for both legs is plotted as the dashed black line.

\subsubsection{Asymmetric leg stiffnesses, $k$}

A linear relationship between the GRF and the length between foot COP and COM can be used to find the the effective leg stiffness the human system conforms to during gait. An inverted pendulum model with a compliant (rather than a rigid) leg has been shown to replicate the GRF behaviour and suggests that human dynamics during walking are analogous to the spring-mass model. In this section, the effect of a person with asymmetric leg stiffnesses and the response that has on the person's gait characteristics is examined using the SLIP model to predict human walking. Asymmetric effective leg stiffnesses, $k$ may be a cause of a person's neuromuscular activity resulting from an injury or using a prosthetic device and represents the linear force-length characteristics of the supporting leg.

Figure 4-11 represents the change in gait characteristics with a different effective leg stiffness value, $k_{B}$, of the leg $B$. At $k_{B}$ of $29.5 \mathrm{kN} / \mathrm{m}$, the model exhibits asymmetric walking and after $30 \mathrm{kN} / \mathrm{m}$, no stability is found for the model. Investigation into these bifurcations is representative of a specific mode which is unlikely to occur, so is 
beyond the scope of this thesis. From Figure 4-11a, it should be noted that although the step length is relatively symmetric, the overall step length seems to increase as $k_{B}$ increases. Figures $4-11 b$ \& $4-11$ c show us that a variation of effective leg stiffness has more of an effect on the step length and walking speed of the unaltered contralateral leg $A$, rather than the altered leg $B$. Research from empirical experiments suggest that leg stiffness increases with walking speed [64]. This seems counter-intuitive to the results from Figure 4-11c, as the stiffer leg has a slower walking speed. However, the spring affects the propulsion energy, which means that a stiffer leg initiates a faster walking velocity for the next step - or the opposite leg.

Figure 4-11d shows that as $k_{B}$ increases, the horizontal impulses become approximately symmetric. However if $k_{B}$ decreases, the impulses from the legs become asymmetric, with the stiffer leg having more propulsion, while the compliant leg contributes to braking - reducing the horizontal body mass momentum during stance.

Looking at the maximum GRF peaks in Figures 4-11e \& 4-11f, asymmetric $k$ values increase the maximum forces of both peaks, with the stiffer leg having higher forces than the compliant leg. If the stiffness of $\operatorname{leg} B, k_{B}$, is reduced by $30 \%$, the compliant leg GRF peaks decrease by $12 \%$ and $17 \%$, while the stiffer unchanged leg $A$ has increased maximum peaks of $23 \%$ and $20 \%$ higher for the first and second peak respectively. It can be seen from Figure 4-12 that the trough of the vertical GRF curve is altered by variation in the contralateral leg stiffness; although leg $B$ has an altered stiffness, the mid-stance GRF remains the same.

\subsubsection{Asymmetric roller radius, $R$}

The human locomotor system conforms to a roll-over shape of $30 \%$ the leg length $[94,2]$. This shape remains constant and doesn't change with walking speed [47], heel height [45] or when carrying extra weight [46]. Figure 4-13 shows the effect of an altered roller radius on the characteristics of gait with the SLIP model. A roller 


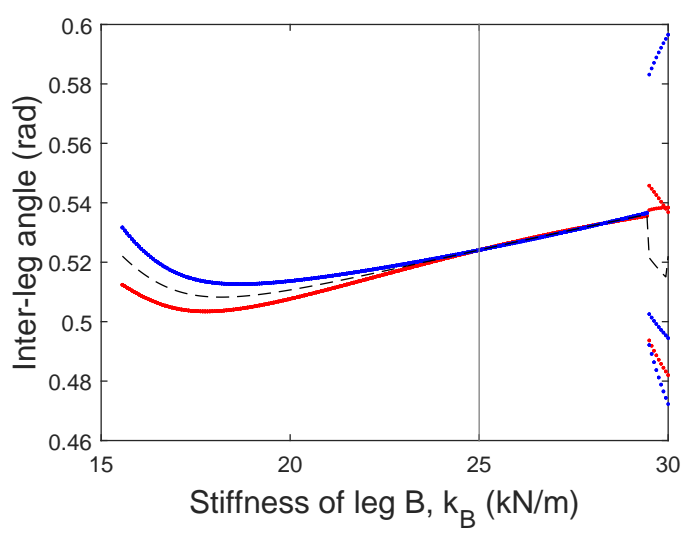

(a)

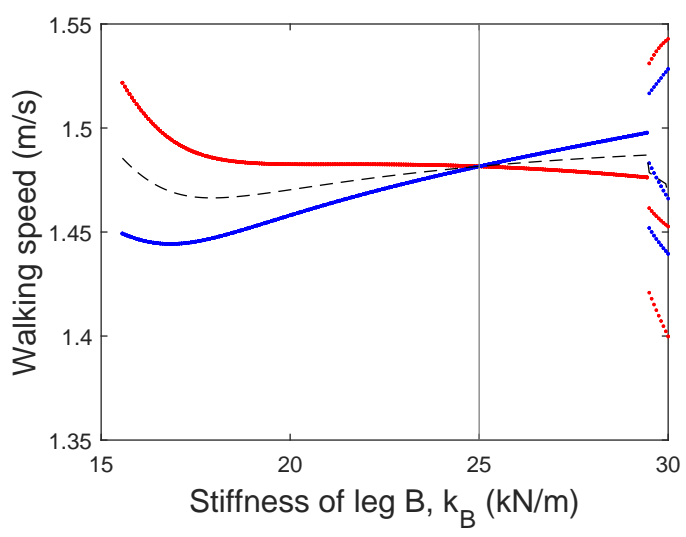

(c)

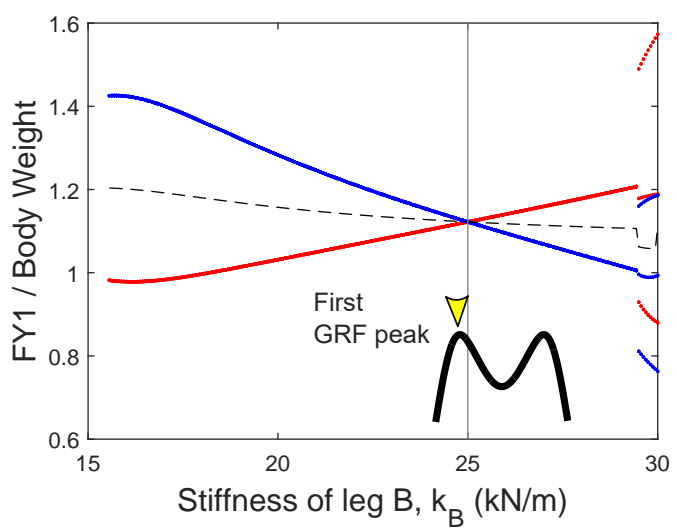

(e)

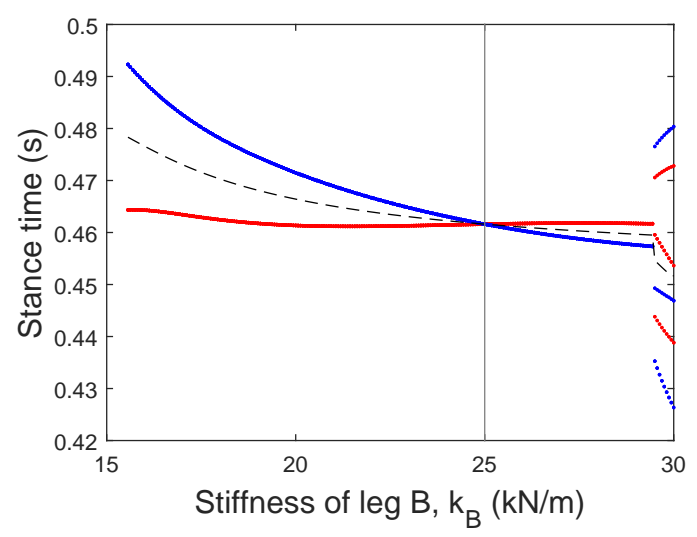

(b)

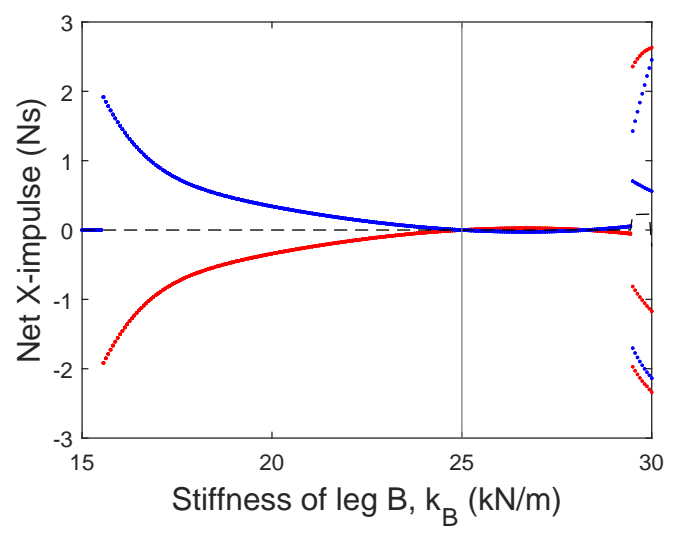

(d)

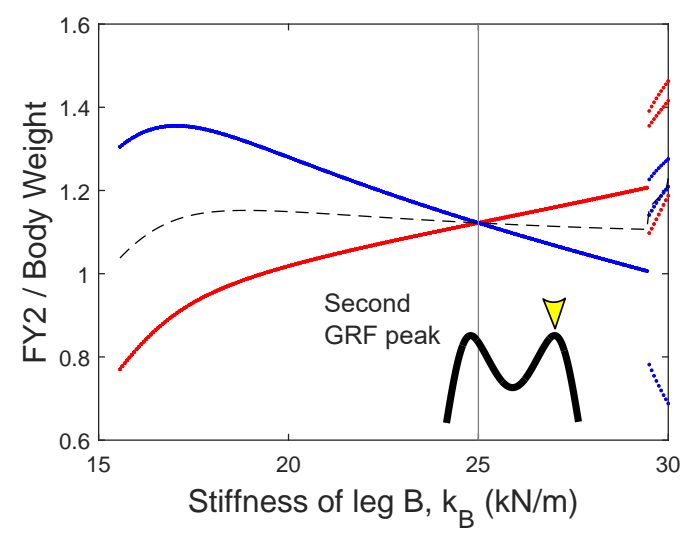

(f)

Figure 4-11: Effect of changing the effective leg stiffness of one leg, $k_{B}$, on the gait characteristics of the SLIP model. The blue points represents gait characteristics while the unaffected leg is the support leg, the red points indicates that the modified leg is the support. The other leg remains at $k_{A}=25 \mathrm{kN} / \mathrm{m}$. 


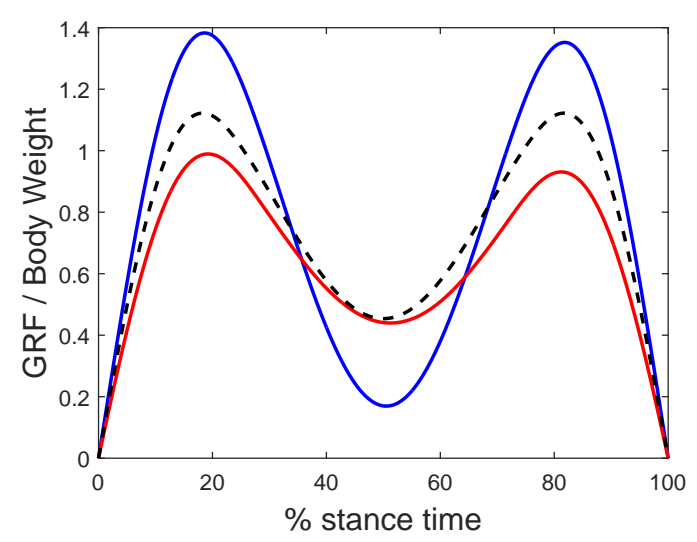

(a) $k_{B}=17.5 \mathrm{kN} / \mathrm{m}$

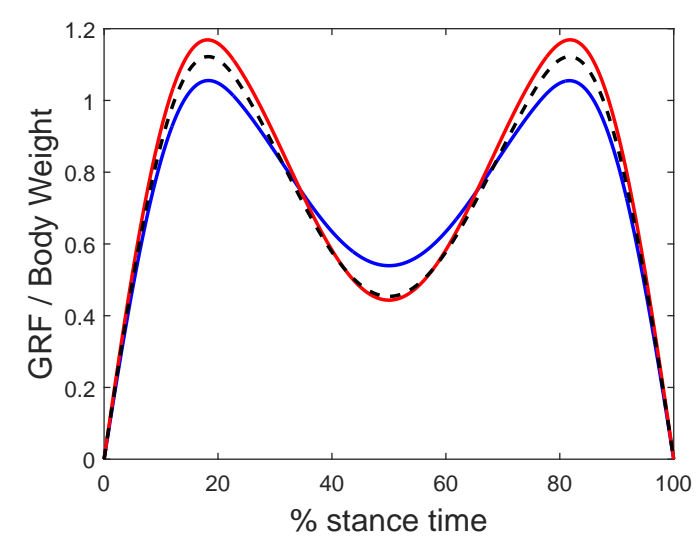

(b) $k_{B}=27.5 \mathrm{kN} / \mathrm{m}$

Figure 4-12: Vertical Ground Reaction Force (GRF) profile for the SLIP walking model with the stiffness of leg $B, k_{B}$, being equal to (a) $17.5 \mathrm{kN} / \mathrm{m}$ and (b) 27.5 $\mathrm{kN} / \mathrm{m}$ (shown by the red line), while the other leg touch-down angle, $k_{A}$, remains at $25 \mathrm{kN} / \mathrm{m}$ (shown by the blue line). The GRF plot for a symmetric model with $k$ being $25 \mathrm{kN} / \mathrm{m}$ for both legs is plotted as the dashed black line.

radius, $R_{B}$, of 0 means that the leg has a point foot. The rocker does not have a defined foot length.

Increasing one leg's roller radius, $R_{B}$ increases the stance time on that leg, which conflicts the prediction seen in the passive dynamic walker in Figure 3-14b from Section 3.4.2. This importantly shows how due to assumptions in these models, they may not accurately match human gait dynamics. Variation in $R_{B}$ seems to affect step length and stance time (Figures 4-13a \& 4-13b) which causes a small variation in walking speed with respect to rocker radius (Figure 4-13c). As $R_{B}$ decreases up to point foot, the stance time and step length of the contralateral unaffected leg increases.

Figures 4-13e \& 4-13f respectively show the first and second maximum vertical GRF peaks in relation to a single changing roller radius, $R_{B}$. A pointed foot has less of a maximum force than the contralateral flatter foot, although the GRF peaks of both limbs increase as $R_{B}$ decreases. This means that utilising rolling contact for the artificial leg has a beneficial effect on the joint loads of the contralateral leg. This is in agreement with experimental evidence from Adamczyk et al. [2]; increasing the 
roller radius decreases the peak vertical GRFs.

\subsection{Discussion of results}

A spring loaded inverted pendulum (SLIP) model has been used to investigate asymmetries in gait mechanics. Using this type of model, it is assumed walking is a mostly passive system; with stability found through a maintained touch-down angle or effective leg stiffness. Amputees spend a longer time on their prosthetic foot than the intact healthy foot $[4,59,91,25]$. The results from this SLIP model suggest that this asymmetry is not likely to be due to the difference in touch-down angle (Figure 4-9b). Although this produces an overall dynamic effect, the simple spring model seems to adapt the walking speed rather than stance time for asymmetries in $\alpha$. Reducing the touch-down angle of leg $B$ by $25 \%$ increases the walking speed of the shallower leg in support by $17 \%$, while the speed of leg $A$ decreased by $28 \%$. A larger step length is responsible for braking/slowing down the body of mass, while a smaller touch-down angle accelerates the COM, propelling the body and speeding up the next step.

An altered effective leg stiffnesses, $k_{B}$, has more of an effect on the gait characteristics of the contralateral non-affected limb $A$, than the altered leg $B$ (Figures 4-11b \& 4-11c). Another benefit of the SLIP model is that it is able to simulate the humanlike M-shaped GRF curve. This model suggests that an asymmetric leg stiffness, $k$, appears to alter the maximum vertical GRF peaks, as can be seen in Figures 4-11e \& 4-11f. Reducing the stiffness of a leg by $30 \%$ decreases the maximum GRF forces of the compliant limb by $12-17 \%$, while increasing the stiffer contralateral leg GRF forces by 20-23\%. This, in turn, may increases impact forces and joint pain for a person with a prosthesis that is not the same effective stiffness as the healthy leg.

The effective rocker radius of the foot also modifies the dynamics of gait. A smaller rocker radius for one foot, $R_{B}$, increases the stance time and step length of the contralateral leg as shown in Figures 4-13b \& 4-13a. Human experiments from 


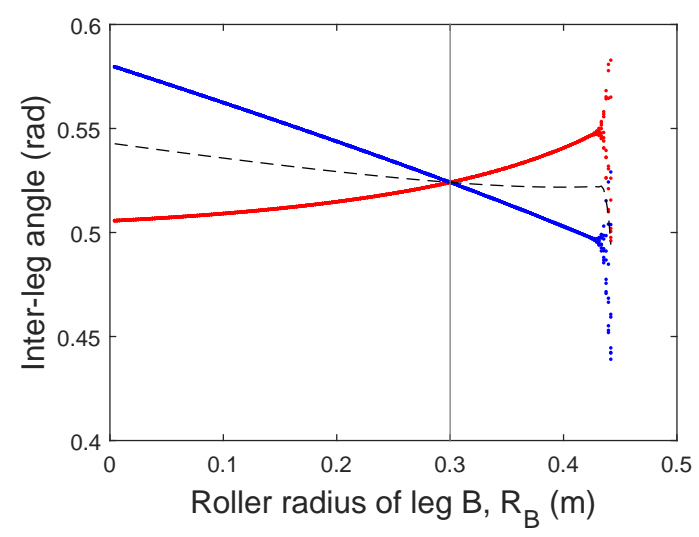

(a)

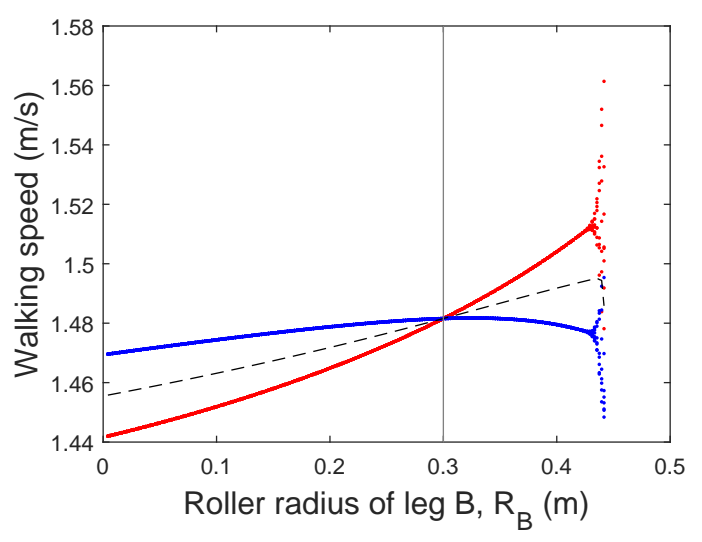

(c)

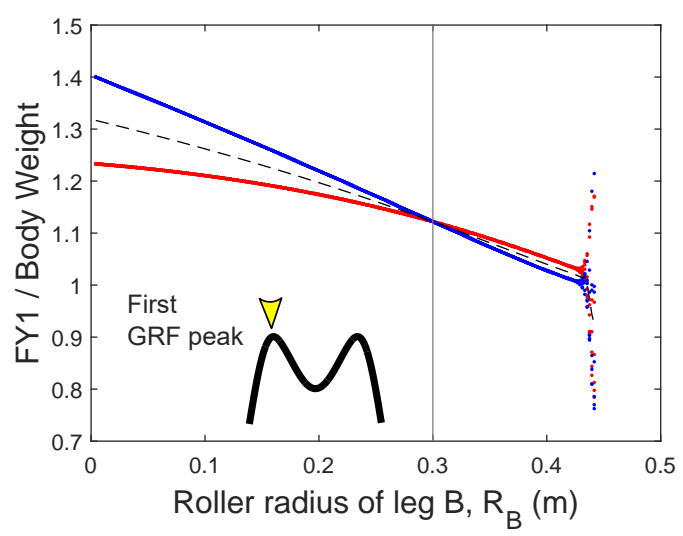

(e)

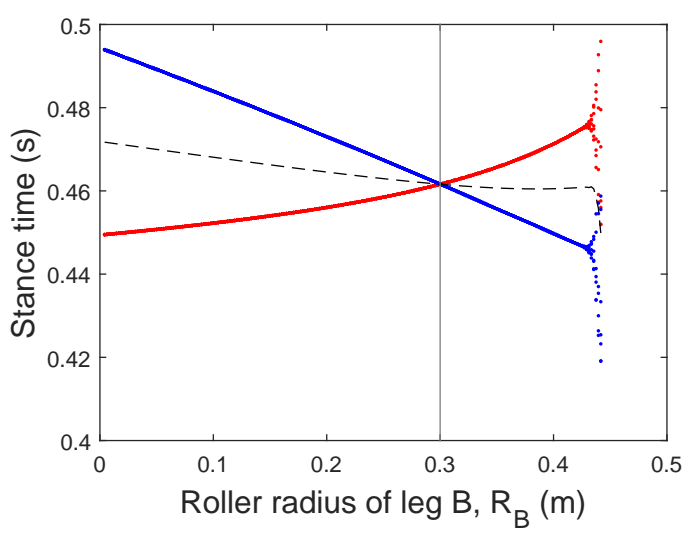

(b)

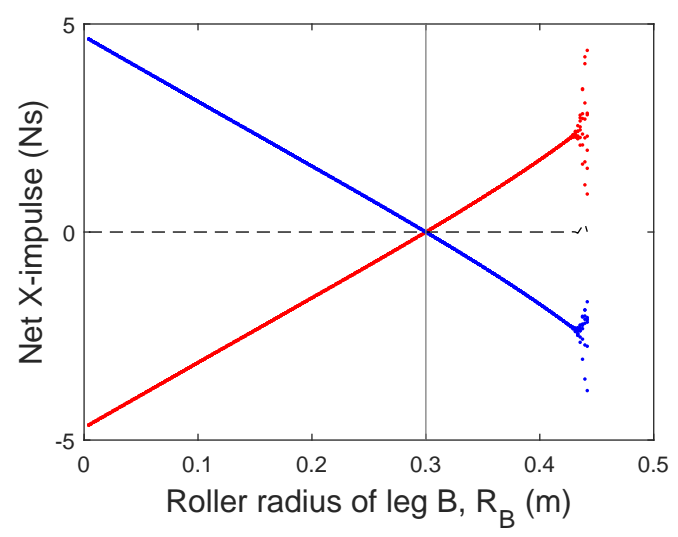

(d)

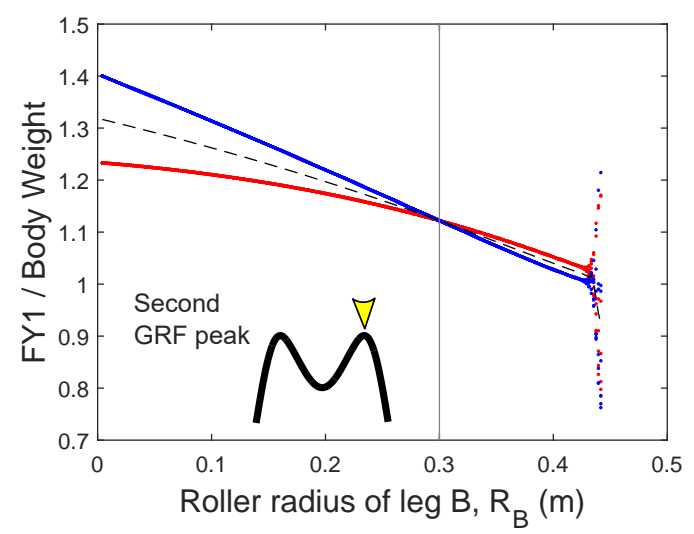

(f)

Figure 4-13: Effect of changing the rocker radius $R_{B}$ of one leg on the gait characteristics of the SLIP model. The blue points represents gait characteristics while the unaffected leg is the support leg, the red points indicates that the modified leg is the support. The other leg remains at $R_{A}=30 \%$ leg length. 


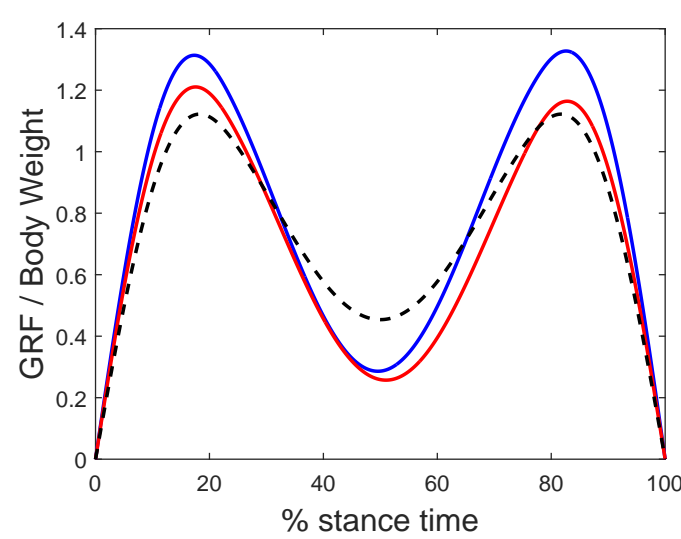

(a) $R_{B}=0.1 \mathrm{~m}$

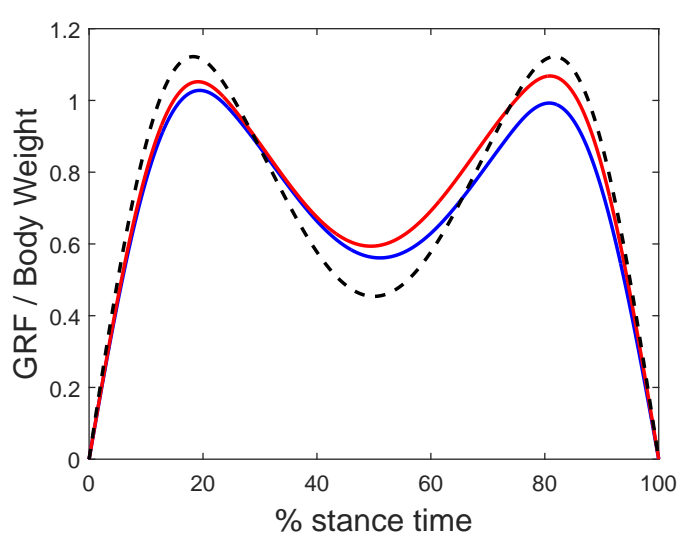

(b) $R_{B}=0.4 \mathrm{~m}$

Figure 4-14: Vertical Ground Reaction Force (GRF) profile for the SLIP walking model with roller radius of leg $B, R_{B}$, being equal to (a) $0.1 \mathrm{~m}$ and (b) $0.4 \mathrm{~m}$ (shown by the red line), while the roller radius, $R_{A}$, remains at $0.3 \mathrm{~m}$ (shown by the blue line). The GRF plot for a symmetric model with $R$ being $0.3 \mathrm{~m}$ for both legs is plotted as the dashed black line.

Adamczyk et al. [2] demonstrate that increasing roller radius of the shoes decreases peak vertical GRFs. Figures 4-13e \& 4-13f show from simulations of the SLIP model, a larger rocker radius for one foot decreases the maximum GRF peaks for both legs.

These results are intended so that understanding is gained from natural dynamics of a simplified system to aid in gait rehabilitation. Prosthetic energy storage and return (ESAR) feet have a similar feature and determine the timing of push-off during gait. However, the SLIP does not model the ballistics of the swing leg dynamically. The next Chapter will extend the models from Chapters $3 \& 4$ to simulate a springloaded passive walker. 


\section{Chapter 5}

\section{Spring loaded passive walker}

The goal of this chapter is to derive a model which incorporates features from both the PW model (Chapter 3) and the SLIP model (Chapter 4). A spring loaded passive walker (SLPW) with masses for each leg is used to investigate the gait characteristics with unbalanced masses or asymmetric leg stiffnesses. The effects of asymmetric gait dynamics on the ballistics of the swing leg and the GRFs are quantified in Section 5.5. This model will ultimately provide insight into these effects to help understand gait rehabilitation from devices and physiotherapy.

\subsection{Equations of motion for spring-loaded passive walker}

The compass model described in Chapter 3 [94] is now adapted to include compliant springs for each leg $[39,113]$ in order to match the ground reaction forces and give insight into the double-support phase of human walking. The model's configuration can be described by the leg angles $\boldsymbol{\theta}=\left[\theta_{1}, \theta_{2}\right]^{T}$ and the leg extensions $\boldsymbol{r}=\left[r_{1}, r_{2}\right]^{T}$ as seen in Figure 5-1. $\theta_{1}$ being the angle of the support leg with the normal to the ground and $\theta_{2}$ being the angle between the support leg and the swinging leg. The 


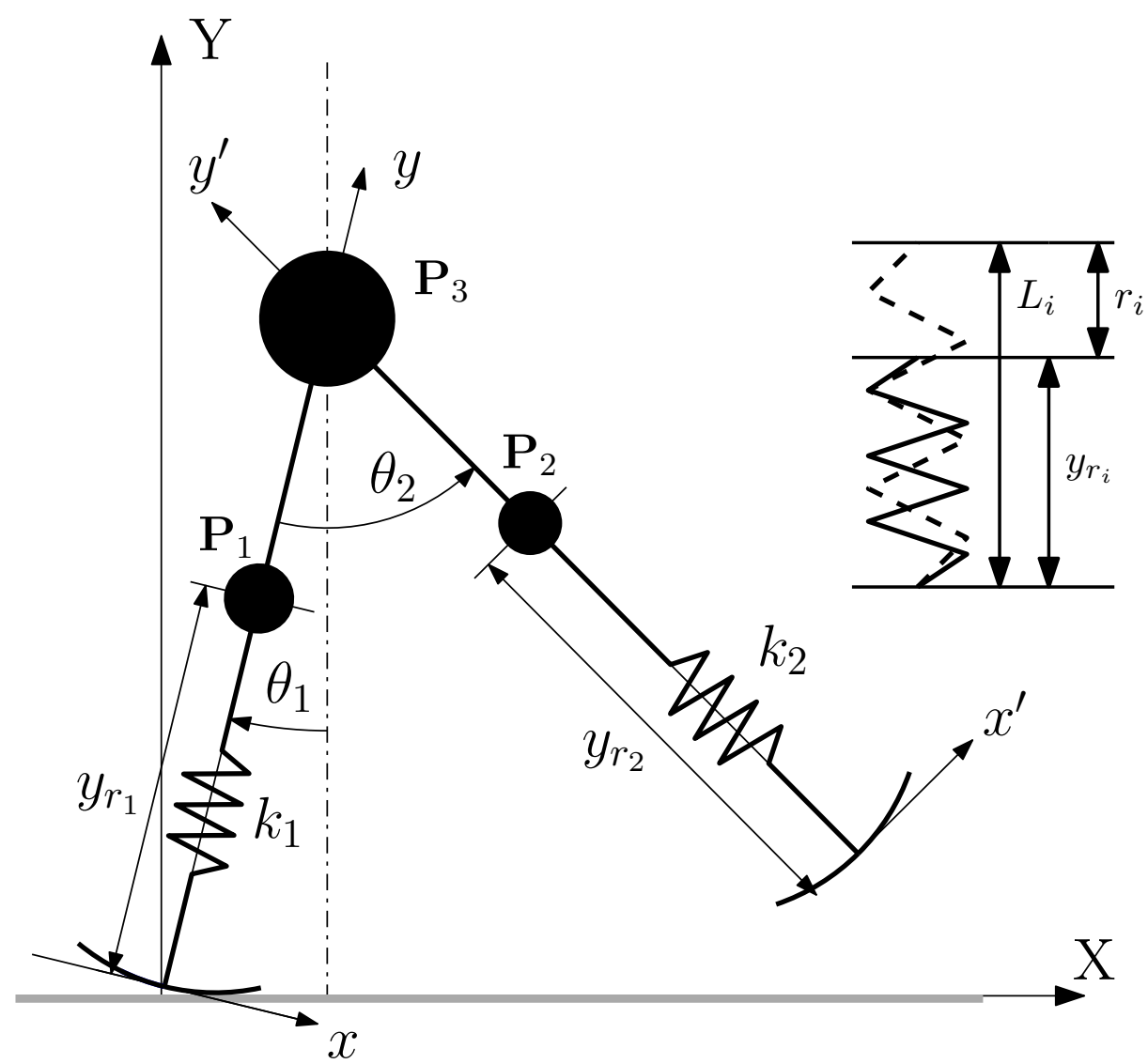

Figure 5-1: Schematic of the Spring Loaded Passive Walker (SLPW) during singlesupport stance phase.

spring extension for the stance leg and swinging leg is respectively $r_{1}$ and $r_{2}$. The state vector $\boldsymbol{q}$ associated with the model is then:

$$
\boldsymbol{q}=[\boldsymbol{\theta}, \boldsymbol{r}]^{T}=\left[\theta_{1}, \theta_{2}, r_{1}, r_{2}\right]^{T}
$$

The model has 3 body masses: a mass $m_{3}$ at the hip at the position $\mathbf{P}_{3}$ and masses $m_{1}$ and $m_{2}$ along each leg at positions $\mathbf{P}_{1}$ and $\mathbf{P}_{2}$ respectively. The locations of mass along the legs are defined in each local coordinate system $\{x, y\}$ and $\left\{x^{\prime}, y^{\prime}\right\}$ as

$$
y_{r_{i}}=L_{i}+r_{i}
$$

for $i=1,2$ and $L_{i}$ is the original mass position of the legs under no load. For each 
leg the total length and position of the hip mass is donated by

$$
y_{H_{i}}=L_{H}+r_{i}
$$

where $L_{H}$ is the original length of the leg. The global coordinates for each mass are

$$
\begin{aligned}
& X_{1}\left(\theta_{1}, r_{1}\right)=-x_{\theta}\left(\theta_{1}\right) \cos \theta_{1}+\left(y_{r_{1}}\left(r_{1}\right)-y_{\theta}\left(\theta_{1}\right)\right) \sin \theta_{1}+s\left(\theta_{1}\right) \\
& Y_{1}\left(\theta_{1}, r_{1}\right)=x_{\theta}\left(\theta_{1}\right) \sin \theta_{1}+\left(y_{r_{1}}\left(r_{1}\right)-y_{\theta}\left(\theta_{1}\right)\right) \cos \theta_{1} \\
& X_{3}\left(\theta_{1}, r_{1}\right)=-x_{\theta}\left(\theta_{1}\right) \cos \theta_{1}+\left(y_{H_{1}}\left(r_{1}\right)-y_{\theta}\left(\theta_{1}\right)\right) \sin \theta_{1}+s\left(\theta_{1}\right) \\
& Y_{3}\left(\theta_{1}, r_{1}\right)=x_{\theta}\left(\theta_{1}\right) \sin \theta_{1}+\left(y_{H_{1}}\left(r_{1}\right)-y_{\theta}\left(\theta_{1}\right)\right) \cos \theta_{1}
\end{aligned}
$$

where functions $x_{\theta}\left(\theta_{1}\right)$ and $y_{\theta}\left(\theta_{1}\right)$ represent the location of COP and are defined from Equations (3.2) and (3.3) using the roll-over function. Also for the swinging leg,

$$
\begin{aligned}
& X_{2}\left(\theta_{1}, \theta_{2}, r_{1}\right)=\left(x_{p_{2}}\left(\theta_{2}\right)-x_{\theta}\left(\theta_{1}\right)\right) \cos \theta_{1}+\left(y_{p_{2}}\left(\theta_{2}, r_{1}\right)-y_{\theta}\left(\theta_{1}\right)\right) \sin \theta_{1}+s\left(\theta_{1}\right) \\
& Y_{2}\left(\theta_{1}, \theta_{2}, r_{1}\right)=-\left(x_{p_{2}}\left(\theta_{2}\right)-x_{\theta}\left(\theta_{1}\right)\right) \sin \theta_{1}+\left(y_{p_{2}}\left(\theta_{2}, r_{1}\right)-y_{\theta}\left(\theta_{1}\right)\right) \cos \theta_{1}
\end{aligned}
$$

where $\left[x_{p_{2}}, y_{p_{2}}\right]$ are the coordinates of point $\mathbf{P}_{2}$ in the $\left\{x_{1}, y_{1}\right\}$ reference frame:

$$
\begin{aligned}
x_{p_{2}}\left(\theta_{2}\right) & =\left(y_{r_{2}}-y_{H_{2}}\right) \sin \theta_{2} \\
y_{p_{2}}\left(\theta_{2}, r_{1}\right) & =\left(y_{r_{2}}-y_{H_{2}}\right) \cos \theta_{2}+y_{H_{1}}\left(r_{1}\right)
\end{aligned}
$$

\subsubsection{Derivation of the equations of motion}

The dynamical equations can be derived from the Euler-Lagrange approach:

$$
\frac{d}{d t}\left(\frac{\partial \mathcal{L}(\boldsymbol{q}, \dot{\boldsymbol{q}})}{\partial \dot{\boldsymbol{q}}}\right)-\frac{\partial \mathcal{L}(\boldsymbol{q}, \dot{\boldsymbol{q}})}{\partial \boldsymbol{q}}=0
$$


where the Lagrangian $\mathcal{L}(\boldsymbol{q}, \dot{\boldsymbol{q}})$ is the difference between kinetic and potential energies $\mathcal{L}(\boldsymbol{q}, \dot{\boldsymbol{q}})=K(\boldsymbol{q}, \dot{\boldsymbol{q}})-V(\boldsymbol{q})$.

$$
\begin{gathered}
K(\boldsymbol{q}, \dot{\boldsymbol{q}})=\frac{1}{2} m_{1}\left|\overrightarrow{\boldsymbol{v}}_{1}\right|^{2}+\frac{1}{2} m_{2}\left|\overrightarrow{\boldsymbol{v}}_{2}\right|^{2}+\frac{1}{2} m_{3}\left|\overrightarrow{\boldsymbol{v}}_{3}\right|^{2} \\
V(\boldsymbol{q})=m_{1} g Y_{1}+m_{2} g Y_{2}+m_{3} g Y_{3}+\frac{1}{2} k_{1} r_{1}{ }^{2}+\frac{1}{2} k_{2} r_{2}{ }^{2}
\end{gathered}
$$

The velocity vectors of each mass $m=1: 3$ are given by

$$
\overrightarrow{\boldsymbol{v}}_{m}=\dot{X}_{m} \vec{\imath}+\dot{Y}_{m} \vec{\jmath}
$$

and the velocities of each mass are given by

$$
\begin{gathered}
\dot{X}_{m}=\frac{\partial X_{m}}{\partial \theta_{1}} \dot{\theta}_{1}+\frac{\partial X_{m}}{\partial r_{1}} \dot{r}_{1}+\frac{\partial X_{m}}{\partial \theta_{2}} \dot{\theta}_{2}+\frac{\partial X_{m}}{\partial r_{2}} \dot{r}_{2} \\
\dot{Y}_{m}=\frac{\partial Y_{m}}{\partial \theta_{1}} \dot{\theta}_{1}+\frac{\partial Y_{m}}{\partial r_{1}} \dot{r}_{1}+\frac{\partial Y_{m}}{\partial \theta_{2}} \dot{\theta}_{2}+\frac{\partial Y_{m}}{\partial r_{2}} \dot{r}_{2}
\end{gathered}
$$

By separating out variables for the state vector, the Kinetic energy (Equation (5.9)) may be rewritten as

$$
\begin{aligned}
K=\frac{1}{2} M_{11} \dot{\theta}_{1}^{2}+\frac{1}{2} M_{22} \dot{\theta}_{2}^{2}+\frac{1}{2} M_{33} \dot{r}_{1}^{2}+\frac{1}{2} M_{44}{\dot{r_{2}}}^{2}+M_{12} \dot{\theta}_{1} \dot{\theta}_{2} \\
+M_{13} \dot{\theta}_{1} \dot{r}_{1}+M_{14} \dot{\theta}_{1} \dot{r}_{2}+M_{23} \dot{\theta}_{2} \dot{r}_{1}+M_{24} \dot{\theta}_{2} \dot{r}_{2}+M_{34} \dot{r}_{1} \dot{r}_{2}
\end{aligned}
$$

Where the values for $M_{i j}$ can be found from:

$$
M_{i j}=\sum_{m=1}^{3} m_{m}\left(\frac{\partial X_{m}}{\partial q_{i}} \frac{\partial X_{m}}{\partial q_{j}}+\frac{\partial Y_{m}}{\partial q_{i}} \frac{\partial Y_{m}}{\partial q_{j}}\right)
$$

Deriving from the Euler-Lagrange Equations (5.8) and inserting into a state-space format:

$$
\boldsymbol{M}(\boldsymbol{q}) \ddot{\boldsymbol{q}}+\boldsymbol{N}(\boldsymbol{q}, \dot{\boldsymbol{q}}) \dot{\boldsymbol{q}}+\boldsymbol{V}^{\prime}(\boldsymbol{q})=0
$$


with values of the matrices $\boldsymbol{N}$ and $\boldsymbol{V}^{\prime}$ defined by

$$
N_{i j}=\sum_{n=1}^{4} \frac{\partial M_{i n}}{\partial q_{j}} \dot{q}_{n}-\frac{1}{2} \sum_{n=1}^{4} \frac{\partial M_{j n}}{\partial q_{i}} \dot{q}_{n}
$$

and

$$
\boldsymbol{V}^{\prime}(\boldsymbol{q})=\left(\begin{array}{llll}
\frac{\partial V}{\partial \theta_{1}} & \frac{\partial V}{\partial \theta_{2}} & \frac{\partial V}{\partial r_{1}} & \frac{\partial V}{\partial r_{2}}
\end{array}\right)^{T}
$$

During single-support stance phase, the swing leg extension, $r_{2}$ is equal to 0 and

$$
\boldsymbol{q}=\left[\theta_{1}, \theta_{2}, r_{1}\right]
$$

This means that during the single-support $\boldsymbol{M}$ and $\boldsymbol{N}$ are $3 \times 3$ matrices and $\boldsymbol{V}^{\prime}$ is a

$3 \times 1$ matrix. No energy is lost at the collision (shown later in Section 5.1.3), therefore there is no need for the walker to traverse down a slope. However, adaptations can be easily made to include a sloped ground and a damper for each leg.

\subsubsection{Double-support phase equations of motion}

The double-support phase initiates as soon as the swinging leg makes contact with the ground. The spring of the leading leg now compresses while the trailing leg decompresses. Double-support lasts until the trailing leg spring completely unloads $\left(r_{2}=0\right)$ and the model takes off into the single-support phase [39].

To adapt the single-support maths to the double-support constraint, Lagrangian constraints are introduced. A schematic of the model during double-support is shown in Figure 5-2. The variable $\phi$ is introduced and represents the angle of the front leg and

$$
\phi=\theta_{1}+\theta_{2}
$$

The point at which the front foot is in contact with the floor $\mathbf{P}_{c}$ is represented by its coordinates $X_{c}$ and $Y_{c}$. The point $\mathbf{P}_{0}$ represents the origin of the $\{X, Y\}$ coordinates 


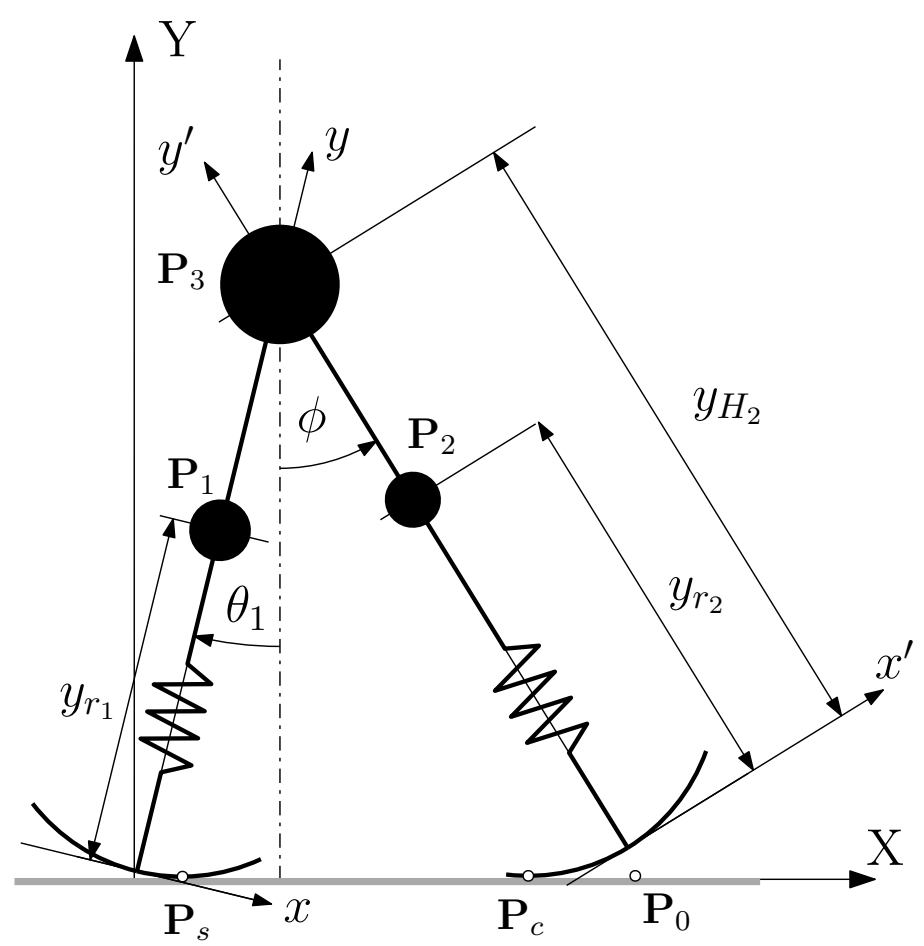

Figure 5-2: Set-up of SLPW during double support phase. The front leg is in contact with the ground at position $\mathbf{P}_{c}$.

of the next step and $\mathbf{P}_{c}=\mathbf{P}_{0}$ when $\phi=0$. The vertical component of the front leg's contact is fixed, so the first constraint is

$$
l=Y_{c}=0
$$

and both feet are allowed to roll with no slipping, so the horizontal constraint is equal to the rolling location of the front foot as visualised in Figure 5-3,

$$
k=X_{c}=X_{0}+s_{2}(\phi)
$$

The walker is now constrained at both feet so with inclusion of the Lagrangian multipliers the Lagrangian becomes

$$
\mathcal{L}(\boldsymbol{q}, \dot{\boldsymbol{q}}, \kappa, \lambda)=K(\boldsymbol{q}, \dot{\boldsymbol{q}})-V(\boldsymbol{q})+\kappa \cdot k(\boldsymbol{q})+\lambda \cdot l(\boldsymbol{q})
$$

So if we add the Lagrangian multipliers, $\kappa$ and $\lambda$, to Equation (5.15) the state space 


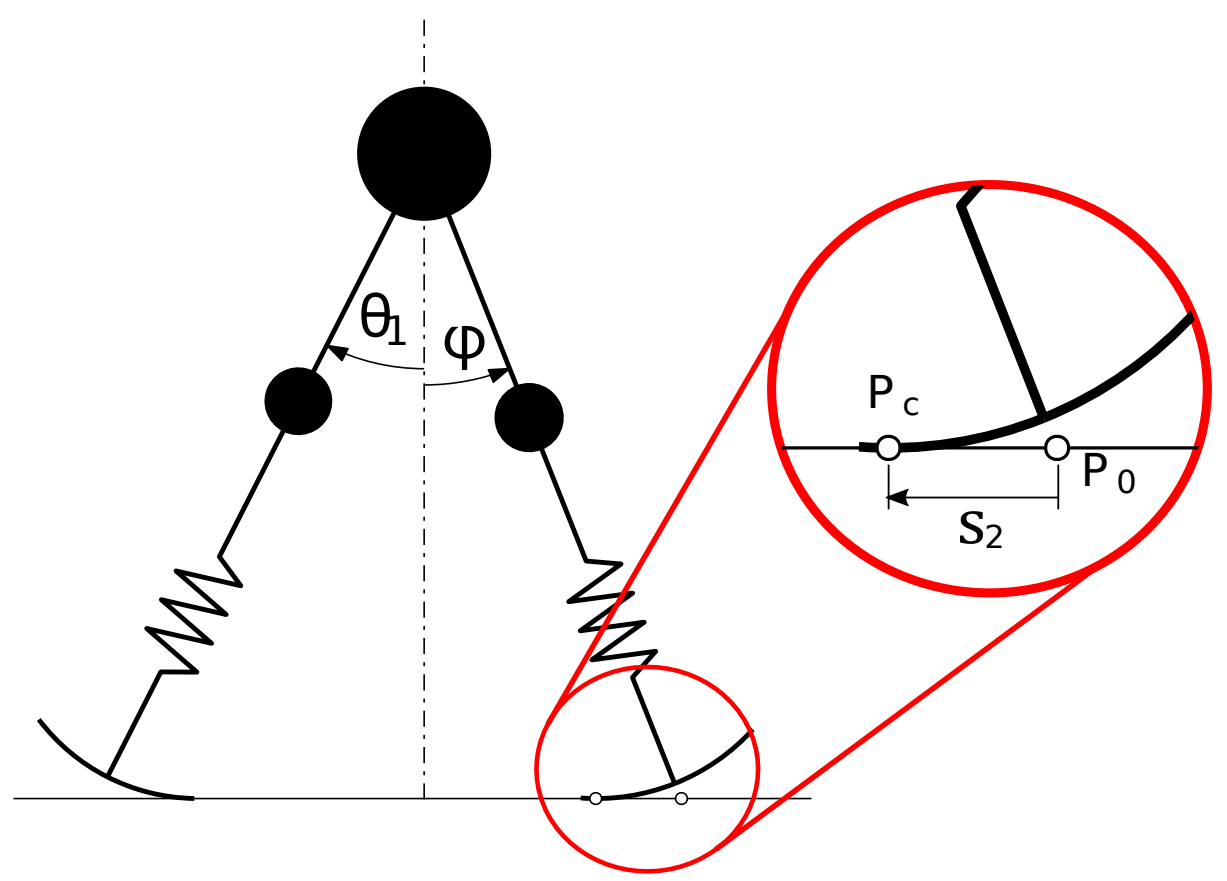

Figure 5-3: Constrained during double-support phase with coordinates evaluated at the rear leg and Lagrangian constraints added to the front foot.

equation during double-support is

$$
\boldsymbol{M}(\boldsymbol{q}) \ddot{\boldsymbol{q}}+\boldsymbol{N}(\boldsymbol{q}, \dot{\boldsymbol{q}}) \dot{\boldsymbol{q}}+\boldsymbol{V}^{\prime}(\boldsymbol{q})-\kappa \cdot \boldsymbol{k}^{\prime}(\boldsymbol{q})-\lambda \cdot \boldsymbol{l}^{\prime}(\boldsymbol{q})=0
$$

There are now 6 unknowns: $\theta_{1}, \theta_{2}, r_{1}, r_{2}, \kappa$ and $\lambda$. Deriving from Equation (5.12), The accelerations of $X_{c}$ and $Y_{c}$ are:

$$
\begin{aligned}
& \ddot{X}_{c}=\sum_{i=1}^{4} \sum_{j=1}^{4} \frac{\partial^{2} X_{c}}{\partial q_{i} \partial q_{j}} \dot{q}_{i} \dot{q}_{j}+\sum_{i=1}^{4} \frac{\partial X_{c}}{\partial q_{i}} \ddot{q}_{i}=\frac{d^{2} s}{d t^{2}}\left(q_{1}, q_{2}\right) \\
& \ddot{Y}_{c}=\sum_{i=1}^{4} \sum_{j=1}^{4} \frac{\partial^{2} Y_{c}}{\partial q_{i} \partial q_{j}} \dot{q}_{i} \dot{q}_{j}+\sum_{i=1}^{4} \frac{\partial Y_{c}}{\partial q_{i}} \ddot{q}_{i}=0
\end{aligned}
$$

and the solution for $\ddot{\boldsymbol{q}}$ can now be resolved from Equations 5.23 and 5.24. 


\subsubsection{Collision mechanics before double-support}

As the swing leg makes contact with the floor, the system transfers from singlesupport to double support. The SLIP model from Chapter 4 comprises of just a hip mass so there is no change of momentum once the front virtual leg makes contact with the ground. In this SLPW model, the front leg has a defined mass, and this constraint leads to a change in momentum as the front swing leg becomes constrained with the ground.

The SLIP model reduces collisional losses between each step due to global elastic behaviour [81]. From the passive walking model, a push-off just before touch-down is hypothesized to reduce collisional losses [73, 23, 15]. For this SLPW model, it is assumed that an impulse is sent from the rear leg immediately before touch-down so that kinetic energy is maintained as the front foot collides with the ground. This leads to the following assumptions:

- Kinetic energy is maintained during the collision.

- Angular momentum of the stance leg and hip mass about the rear leg contact is conserved.

- The horizontal front leg contact velocity, $\dot{X}_{c}$, is equal to the front leg rolling velocity $\dot{s}_{2}$ after the collision.

- The vertical front leg contact velocity, $\dot{Y}_{c}$, is equal to zero after the collision.

or to express this into equation form:

- $K^{-}=K+$

- $m_{1}\left|\overrightarrow{\boldsymbol{v}}_{1}\right|^{-}+m_{3}\left|\overrightarrow{\boldsymbol{v}}_{3}\right|^{-}=m_{1}\left|\overrightarrow{\boldsymbol{v}}_{1}\right|^{+}+m_{3}\left|\overrightarrow{\boldsymbol{v}}_{3}\right|^{+}$

- $\dot{X}_{c}^{+}=\dot{s}_{2}(\phi, \dot{\phi})$

- $\dot{Y}_{c}^{+}=0$ 


\subsubsection{Numerical implementation}

The SLPW model comprises of hybrid dynamics consisting of single-support and double-support phases. As with the SLIP model, the model transfers to single-support when the rear leg extends and loses contact with the floor (when $r_{1}=0$ ). When the swinging leg makes contact with the floor again and is retracting (i.e. $\dot{\theta}_{2}>0$ as with PW model) the touch-down event occurs. Momentum transfer ensures that mechanical energy and angular momentum is maintained with no foot slipping and the model's dynamics alters back to double-support at the start of the next step.

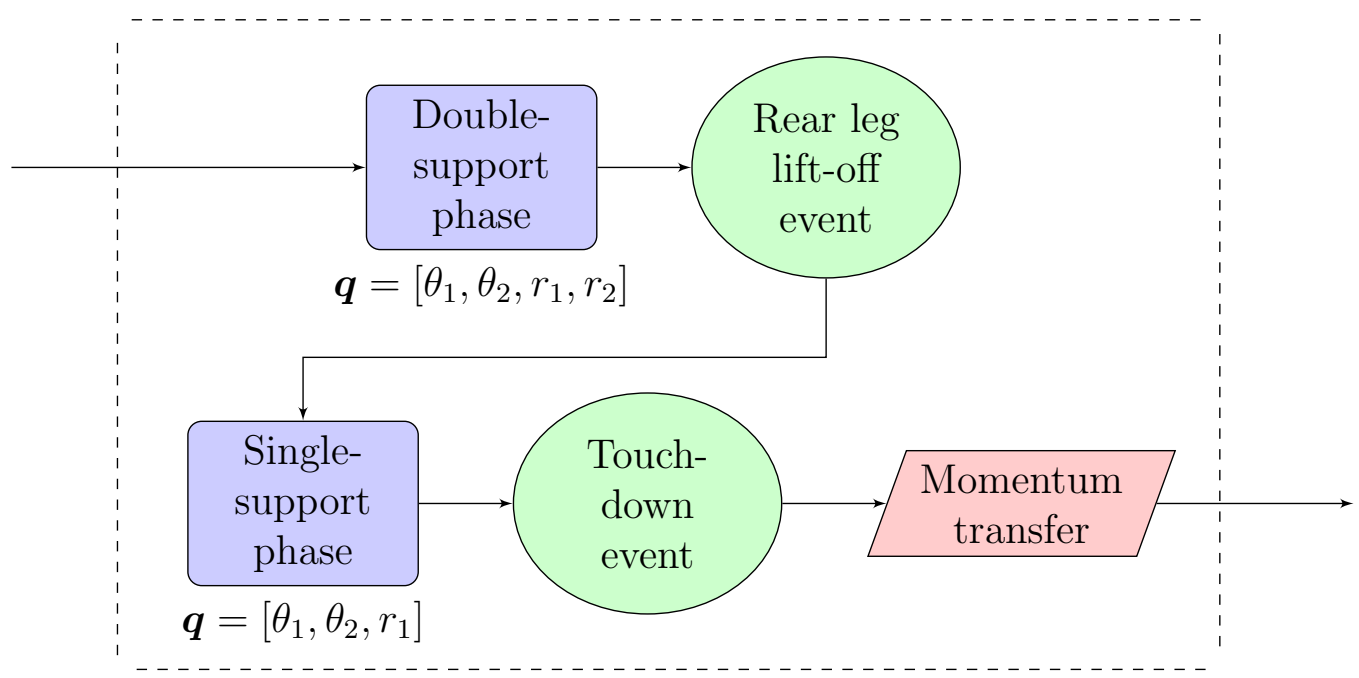

Figure 5-4: Flowchart of a complete walking step in the SLPW model. Can be integrated as the 'Complete Walking Step' block in Figure 3-5.

\subsection{Limit cycle of stable walking solution}

Figure 5-5a show that with a random set of initial conditions, the model can settle to a stable walking cycle within a few steps. The leg kinetics and kinematics during a walking step can be visualised with the help of a phase-plane diagram. Figure 5-5b shows the evolution of the walker at a stable, symmetric gait pattern. This phaseplane diagram can provide important insight into the characteristics of the dynamics 
of walking. During swing phase, the swing leg extension, $r_{2}$, and velocity, $\dot{r}_{2}$, are locked at zero until the swing leg makes contact with the ground again.

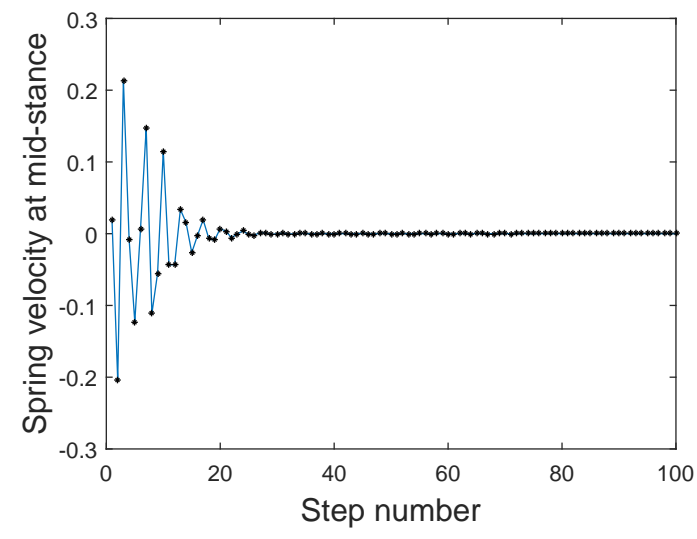

(a)

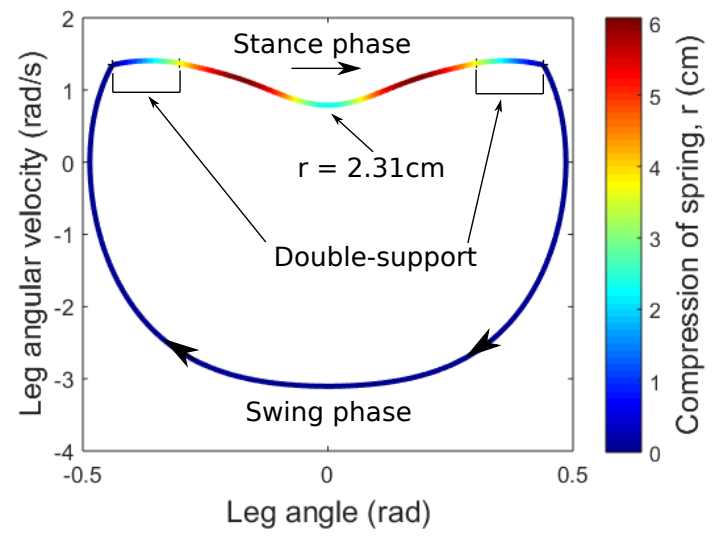

(b)

Figure 5-5: (a) Stable sequence walking up to 100 steps shows that the model converges from a random set of initial conditions. (b) Phase plane shows a full cycle of model for both steps.

\subsection{Parameter domain for SLPW model}

While parameters such as body mass, $M$, and leg length, $L$, are fixed for an individual, Mechanical Energy (ME) and effective leg stiffness, $k$, are variable with walking speed. Figure 5-6 shows the that stable solutions are found for combinations of ME and $k$. Domains may be separated by the number of 'peaks' in the ground reaction force (GRF) curve. In normal human walking, the GRF curved is M-shaped, having 2

defined 'peaks' as shown in Figure 2-4. For the following results of investigations into asymmetry, only results with 2 peaks will be considered. 


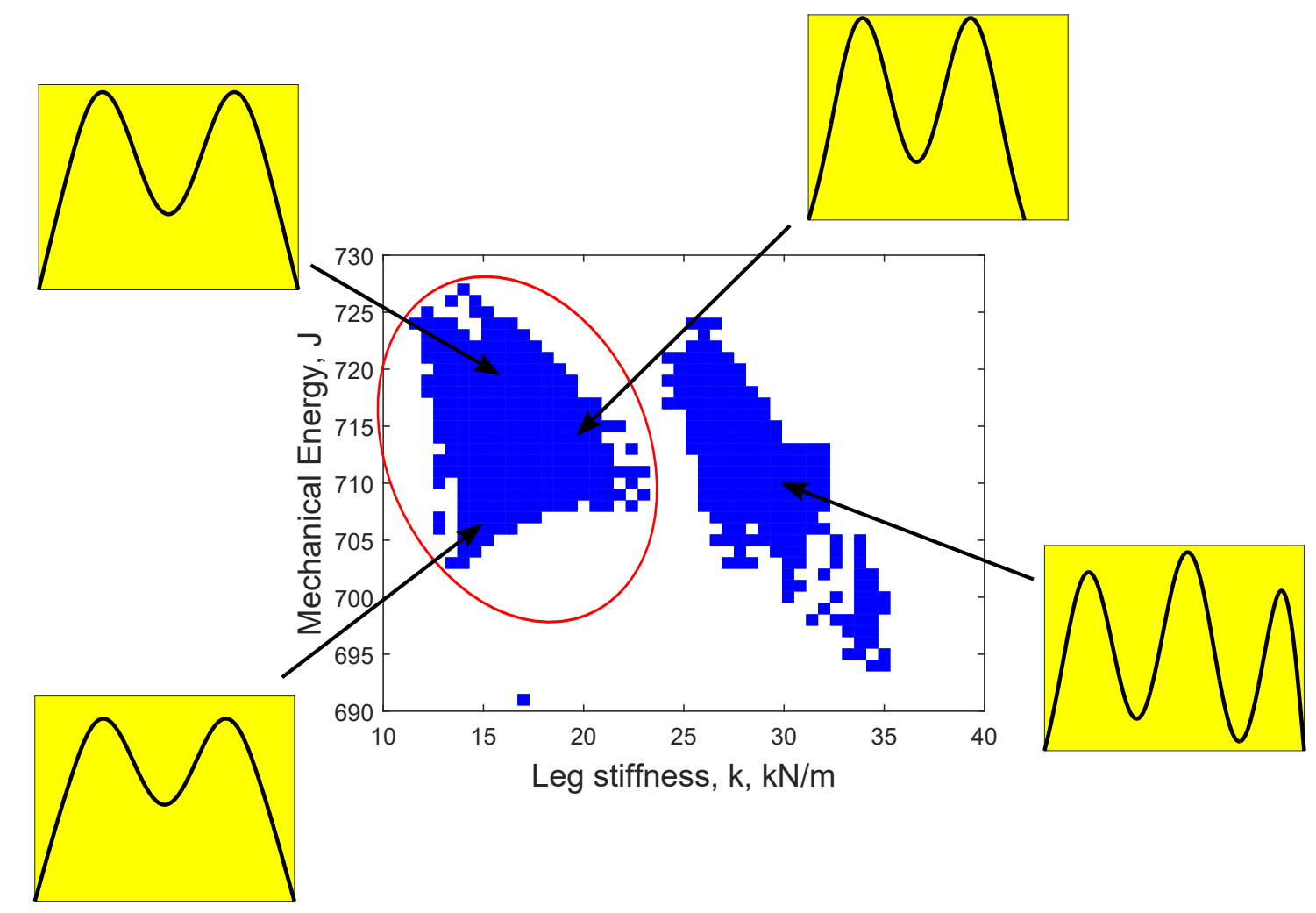

Figure 5-6: Parameter Domain showing stable walking conditions at combinations of effective leg stiffness and mechanical energy. Outside plots represent the GRF pattern exhibiting two to six peaks seen in this parameter domain. Only two-peak GRF patterns (region within red ellipse) are considered in this chapter.

\subsection{Model parameters}

As with the previous models, the model specifications are selected to be similar to human walking parameters. Values for the total body mass, $M$, the leg mass ratio, $\mu$, and leg length ratio, $\beta$, are estimated using anthropomorphic data from Ohio [105]. Effective leg stiffness is estimated from human gait data by studying the force-length curves [81] or by looking into the parameter space for an appropriate walking speed. The roll-over shape is replicated using a rocking contact of $30 \%$ leg length [47]. Table 5.1 outlines the standard parameters used in the following sections, unless a specific variable is stated to have been altered to investigate asymmetry. 


\begin{tabular}{lcc}
\hline Name & Notation & Value \\
\hline Natural leg length & $L$ & $1 \mathrm{~m}$ \\
Total mass & $\sum m$ & $80 \mathrm{~kg}$ \\
Length ratio & $\beta$ & 0.6 \\
Mass ratio & $\mu$ & 3.6 \\
Radius of gyration/leg length & $\mathrm{R}$ & 0.3 \\
Effective leg stiffness & $k$ & $18 \mathrm{kN} / \mathrm{m}$ \\
Mechanical Energy & $\mathrm{ME}$ & $710 \mathrm{~J}$ \\
\hline
\end{tabular}

Table 5.1: Standard parameters used for the SLPW model.

\subsection{Investigating asymmetry in the spring-loaded passive walker}

To gain understanding of this model and make comparisons with the human locomotor system, gait characteristics are defined as follows: (a) inter-leg angle, the angle made between the legs, $\theta_{2}$, at the end of the step at heel-strike; (b) stance time, the total time the leg is in contact with the ground for each step (i.e. from heel-strike to toe-off); (c) walking speed, distance per unit time from touch-down to opposite touch-down and (d) net horizontal impulse from the leg during stance phase. The vertical GRF has also been quantified by the maximum (e) first vGRF peak and (f) second vGRF peak observed for each leg.

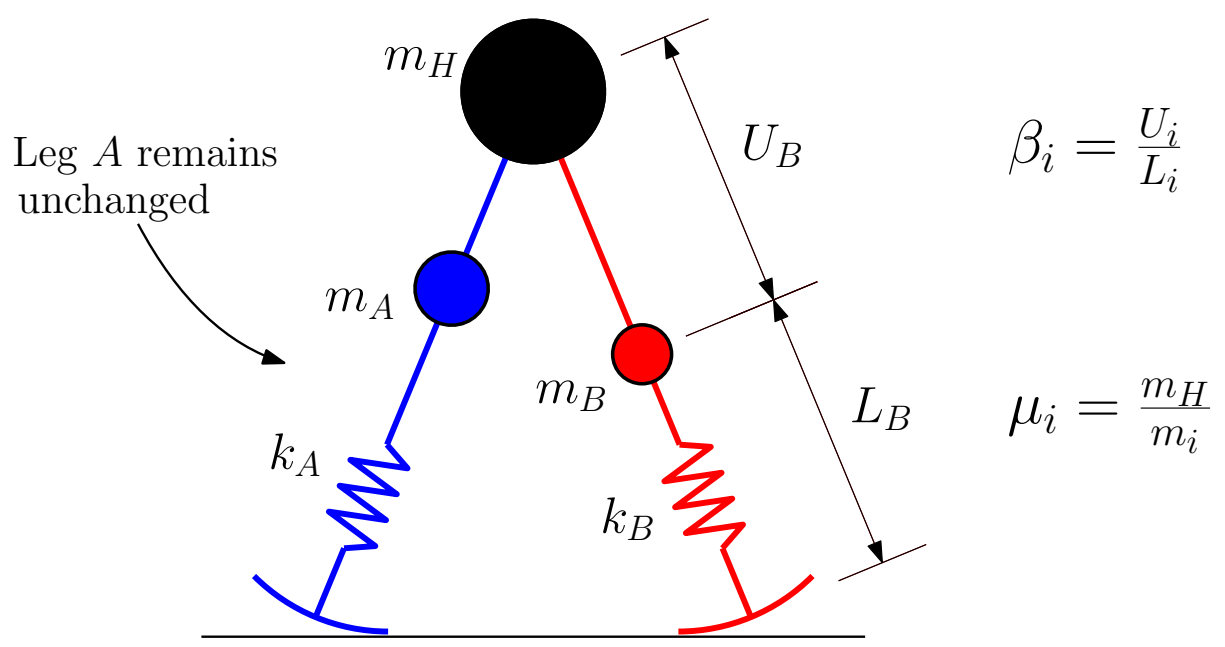

Figure 5-7: Length ratio $\left(\beta_{A}=\frac{U_{A}}{L_{A}}\right)$, mass ratio $\left(\mu_{A}=\frac{m_{H}}{m_{A}}\right)$ and leg stiffness of leg $A, k_{A}$, remains the same while $\beta_{B}, \mu_{B}$ or $k_{B}$ for leg $B$ varies. 
Figure 5-7 represents the asymmetric SLPW used to investigate asymmetry. The parameters for leg $B$ are changed independently, while the parameters for leg $A$ remain constant for each section in this chapter with the unchanged values found in Table 5.1.

\subsubsection{Asymmetric leg masses}

The effects of asymmetric leg masses on the dynamics of gait have been shown in the PW model in Section 3.4.1, however the current SLPW model more accurately predicts the double-support time and simulates the effect on the GRFs. Prosthetic feet generally weigh about $1 / 4$ of the weight of the effective physiological leg, with energy expenditure increasing if it is loaded to the same value [36]. The mass ratio, $\beta$, of a transtibial amputee is approximately 4.7 , while the COM position ratio is estimated to be 0.38. Other devices such as bionic prosthetics or exoskeletons can make the limb weigh a lot more than average. With this model, the effect of altered mass and mass position can be seen in the passive dynamics of gait.

\section{Asymmetric leg mass ratio, $\mu$}

The mass ratio, $\mu$, is the ratio of hip mass to leg mass. A higher mass ratio of the leg will lead to a lighter leg. Leg $A$ has a constant mass ratio, $\mu_{A}$, of 3.6. Figure 5-8 shows the evolution of gait parameters with one leg altered to evaluate the asymmetry of gait. A minimum of mass ratio of leg $B, \mu_{B}$, of 3.49 is achieved before the model performs chaotically and stable walking below this region is not found.

Figure 5-8c shows the evolution of walking speed for both stance times as the mass ratio of leg $B, \mu_{B}$, increases. The model is not robust enough to deal with a large variation of asymmetric $\mu$ as compared to the rigid passive walker in Chapter 3 . The model retains symmetry from alteration of $\mu_{B}$, however the total walking speed increases as one of the legs becomes lighter. The gait characteristics for the rigid 
passive walking model outlined in Section 3.4.1 are not symmetric. It is important to note that a walking model with compliant legs reacts differently to asymmetries in leg mass ratios compared with the previous PW model in Chapter 3.

The maximum vertical GRF peaks, shown in Figures 5-8e \& 5-8f, do not change much until the one leg reaches a mass ratio, $\mu_{B}$ of 3.78 and the net horizontal impulse starts to become rapidly asymmetric (5-8d). In this model, the horizontal impulse from a lighter leg increases. It may be interesting to investigate the stability when a lighter foot cannot provide the necessary impulse, for example with passive prosthetic feet.

\section{Asymmetric leg mass position ratio, $\beta$}

The SLPW is comprised of a hip mass and two leg masses. The COM of the leg mass is at a position along the leg, $L_{i}$, shown in Figure 5-7. A higher leg position ratio, $\beta_{B}$, leads to a lower position of mass, $y_{B}$. Changing the position of mass of the leg does not seem to change the stance time appreciably as can be seen in Figure 5-9b. However, from Figures 5-9a \& 5-9c it can be seen that the inter-leg angle and walking speed both increase with a lower position of mass. Hekmatfard et al. [50] investigated the effects of prosthetic mass distribution to and found that with a higher $\beta_{B}$, the speed and step lengths of the prosthetic were significantly larger than those of the intact limb which is in alignment from the results of these simulations.

Figure 5-9d represents the net horizontal impulse from each leg. A lower leg mass contributes more to propulsion, while a higher leg mass contributes to braking. Figures 5-9e and 5-9f display the first and second vGRF peaks, FY1 and FY2, respectively. A larger value for $\beta_{B}$ increases FY1 of leg $B$ more than leg $A$. Increasing $\beta_{B}$ also increases the maximum FY2 for both legs. Figure 5-10 shows the vGRF profiles for both legs with an altered COM location of $\operatorname{leg} B, \beta_{B}$ of 0.67 . The first and second peak of the altered leg vGRF profile increased by $15.4 \%$ and $7.7 \%$ respectively. The unaltered leg $A$ 's vGRF peaks (with $\beta_{A}$ remaining at 0.60 ) increased by $4.7 \%$ and 


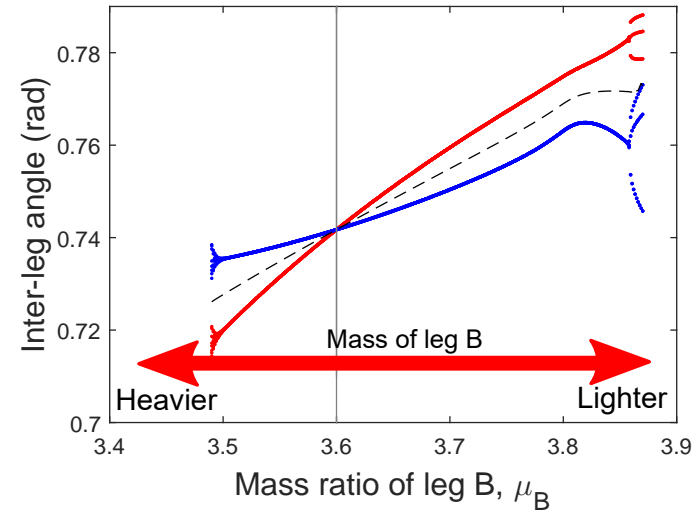

(a)

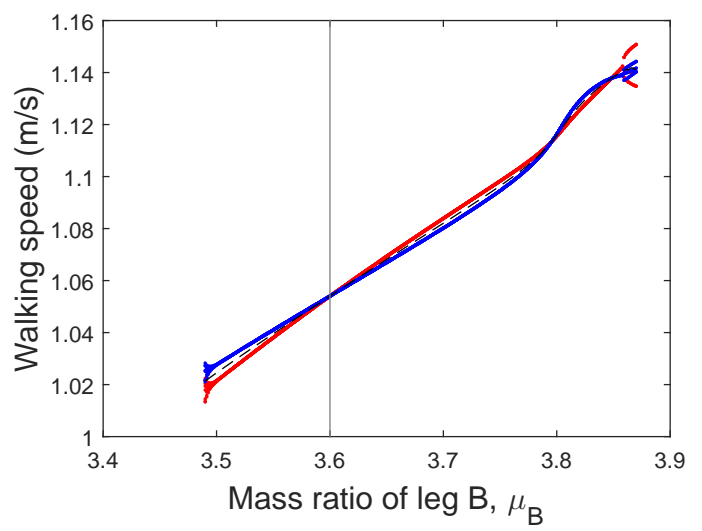

(c)

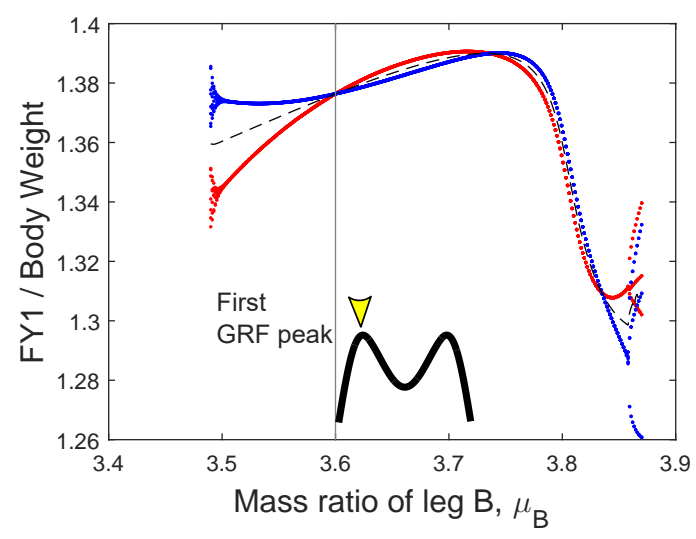

(e)

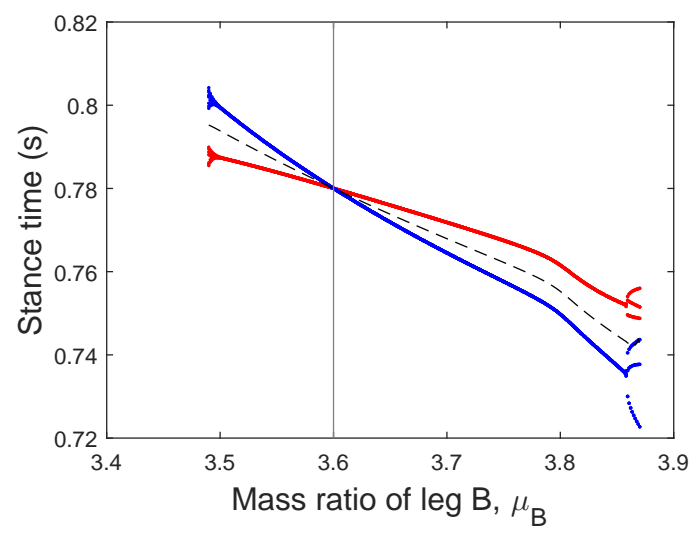

(b)

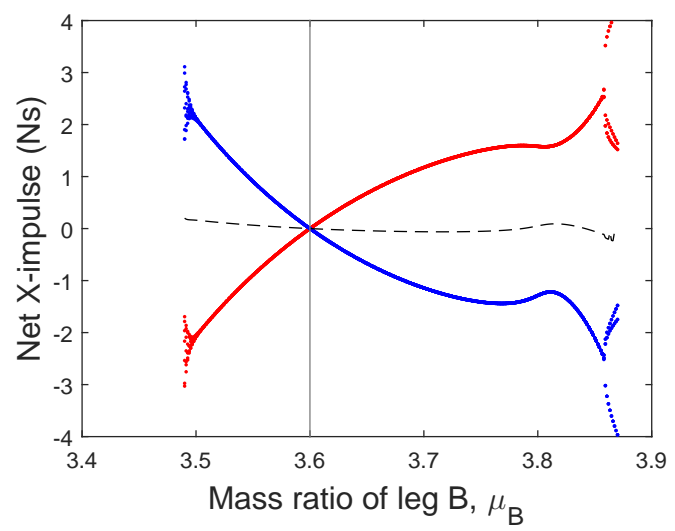

(d)

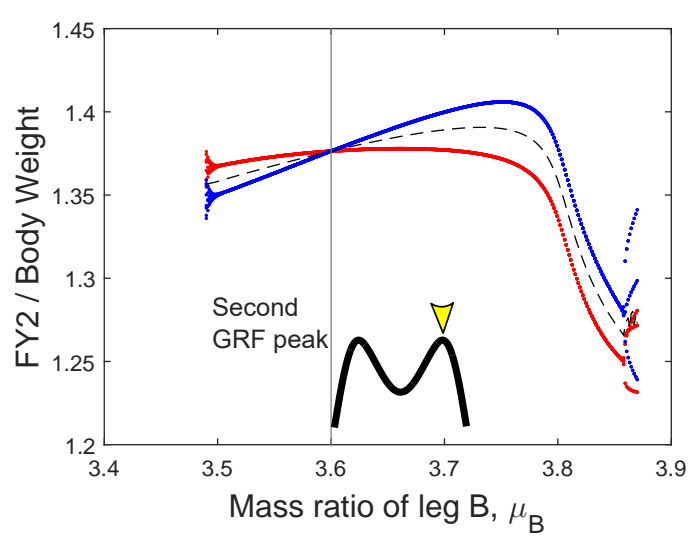

(f)

Figure 5-8: Effect of changing the effective leg mass ratio, $\mu_{B}$, of one leg on the gait characteristics of the SLPW model. The blue points represents gait characteristics while the unaffected leg is the support leg, the red points indicates that the modified leg is the support. The other leg remains at $\mu_{A}=3.6$ 
$11.2 \%$ respectively; comparing to the symmetric unaltered model.

This model becomes unstable below $\beta_{B}$ values of 0.56 . Although there are limitations of the model to predict below this point (while keeping the other variables the same), the type of asymmetric behaviour is clearly seen. It is not known how a person would adapt other variables - such as ME and $k$ - to maintain stable walking conditions with an altered $\beta$.

\subsubsection{Asymmetric leg stiffness, $k$}

Effective leg stiffness in human walking is characterised by the force-length curve during gait. The length being the distance between the centre of mass (COM) and centre of pressure (COP) during stance phase and the ground reaction corce (GRF) obtained via force plates. A spring-loaded inverted pendulum model is able to simulate the double-hump GRF as seen in human walking with a linear relationship for the spring stiffness providing a good estimation at normal walking speeds [81].

By keeping all of the other variables the same, and just changing the leg stiffness of leg $B, k_{B}$, the effect on gait characteristics such as walking speed, step length and peak vertical GRF forces are investigated to gain understanding of asymmetric gait dynamics resulting in Figure 5-11. From Figures 5-11a \& 5-11b, it can be seen that a larger $k_{B}$, induces a smaller step length for leg $B$ and a longer stance time for leg $A$. This results in a slower walking speed for the stiffer limb. A stiffer leg reduces the maximum vertical GRF peaks, while increasing the vGRF for the more compliant contralateral leg as can be seen in Figures 5-11e \& 5-11f. These forces may increase loading on the joints and cause further discomfort not just for the affected leg but also for the unaffected leg and may be why amputees suffer from higher rates of osteoarthritis on their sound limb $[27,103,114]$. Figure 5-12a represents the vertical GRF profile with $k_{B}$ equal to $16 \mathrm{kN} / \mathrm{m}$. For the altered leg $B$, the first and second peaks increase by $7.3 \%$ and $4.9 \%$ respectively, while the vGRF peaks for leg 


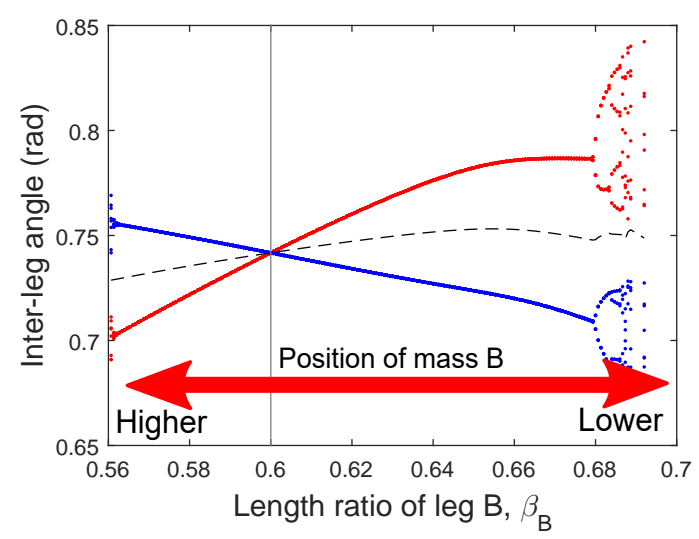

(a)

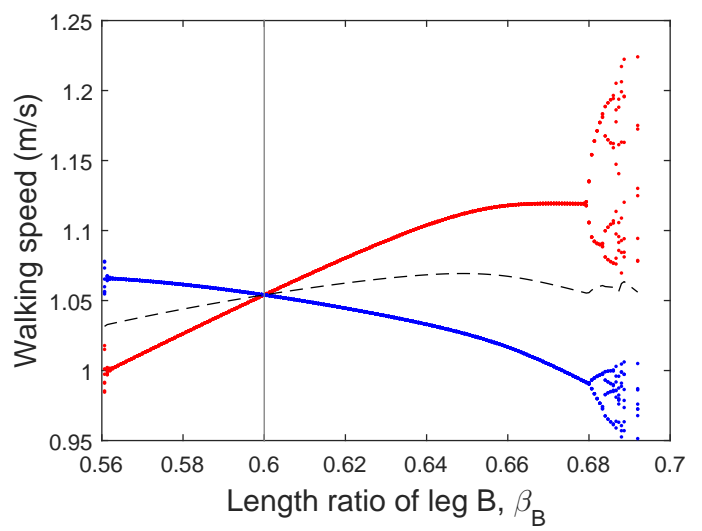

(c)

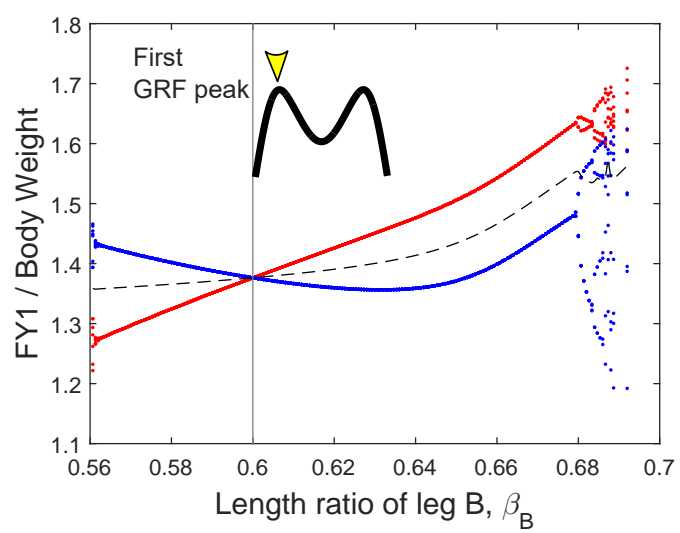

(e)

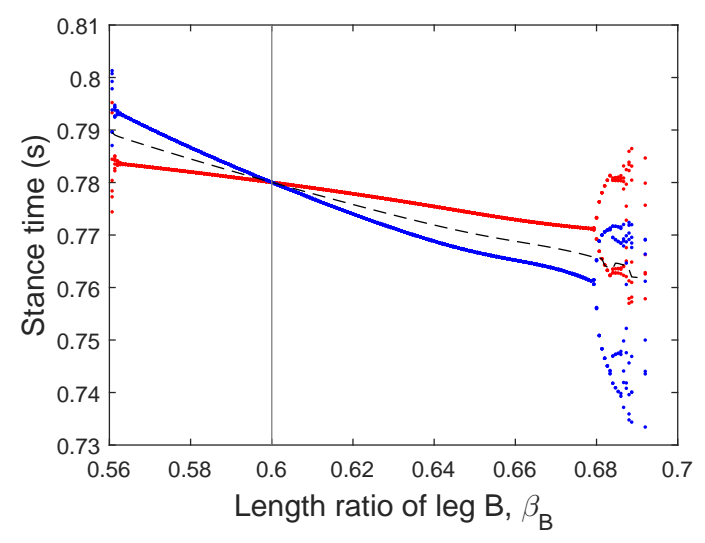

(b)

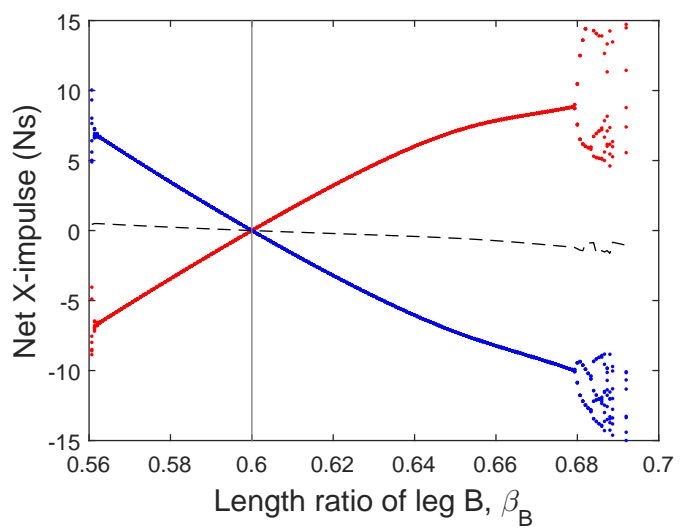

(d)

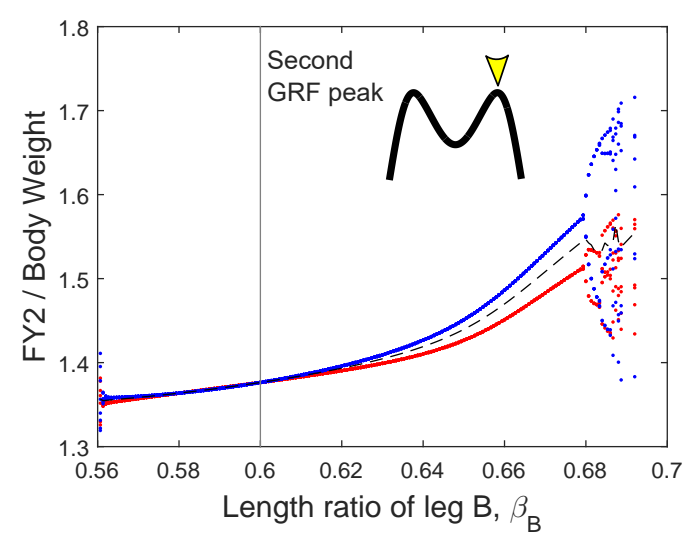

(f)

Figure 5-9: Effect of changing the effective leg mass position ratio, $\beta_{B}$, of one leg on the gait characteristics of the SLPW model. The blue points represents gait characteristics while the unaffected leg is the support leg, the red points indicates that the modified leg is the support. The other leg remains at $\beta_{A}=0.6$ 


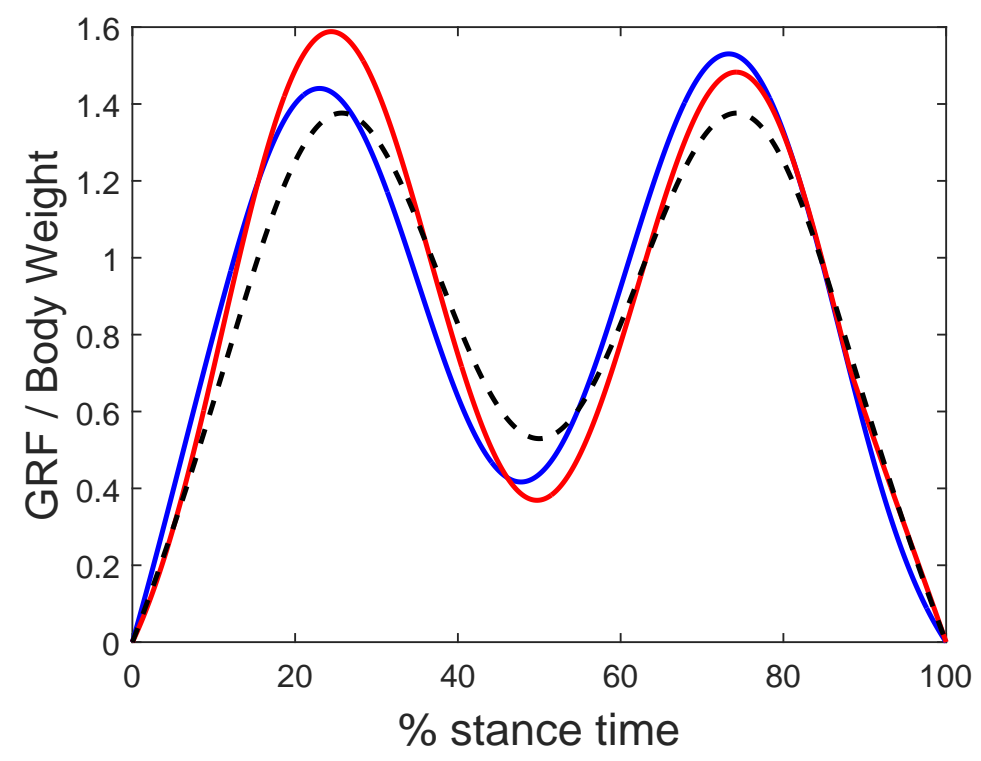

Figure 5-10: Vertical ground reaction force (GRF) profile for the SLPW walking model with COM ratio of leg $B, \beta_{B}$, being equal to 0.67 (shown by the red line), while the other leg COM ratio, $\beta_{A}$, remains at 0.60 (shown by the blue line). The GRF plot for a symmetric model with $\beta$ being 0.60 for both legs is plotted as the dashed black line.

$A$ decrease by $10.3 \%$ and $6.9 \%$. Similarly, increasing $k_{B}$ to $20 \mathrm{kN} / \mathrm{m}$ increases leg $A$ 's vertical GRF peaks by $9.9 \%$ and $9.0 \%$ while decreasing leg $B$ 's first and second peaks by $1.9 \%$ and $0.7 \%$ respectively.

Interestingly, these results contradict the results from the SLIP model in the previous chapter as shown in Figure 4-11. The SLIP model ignores the ballistics of the swing leg, and instead assumes that the swing leg makes contact with the ground at a constant defined touch-down angle, $\alpha$. These results show that spring stiffness has an effect on the swing leg dynamics and needs to be considered; with a more compliant limb having a greater push-off, a larger step length and a shorter swing time. 


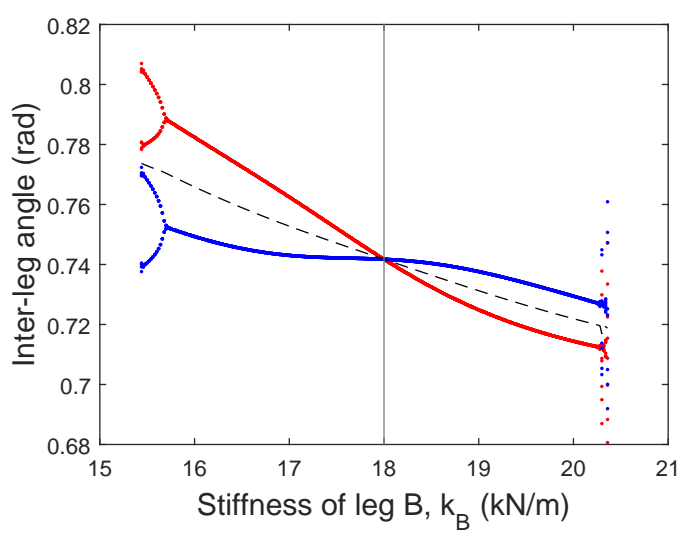

(a)

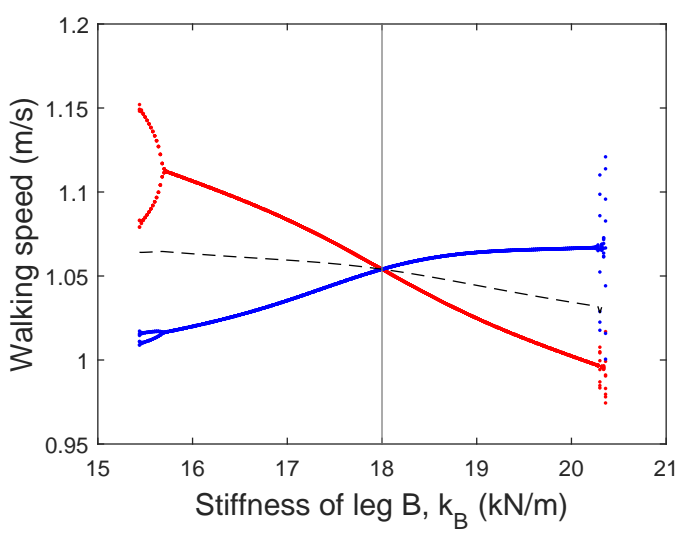

(c)

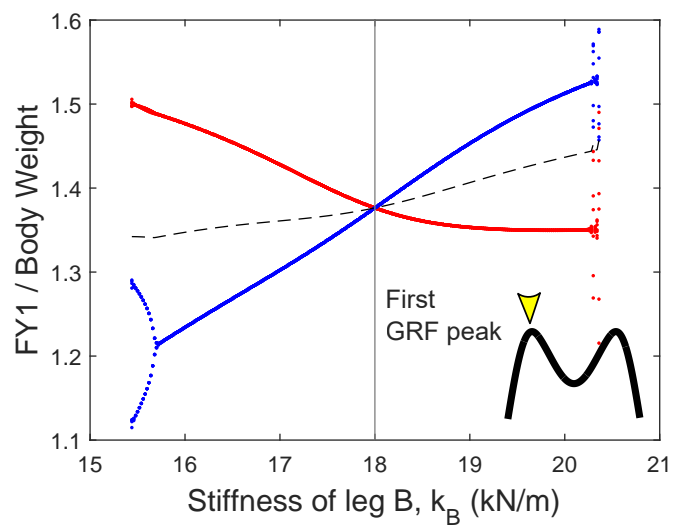

(e)

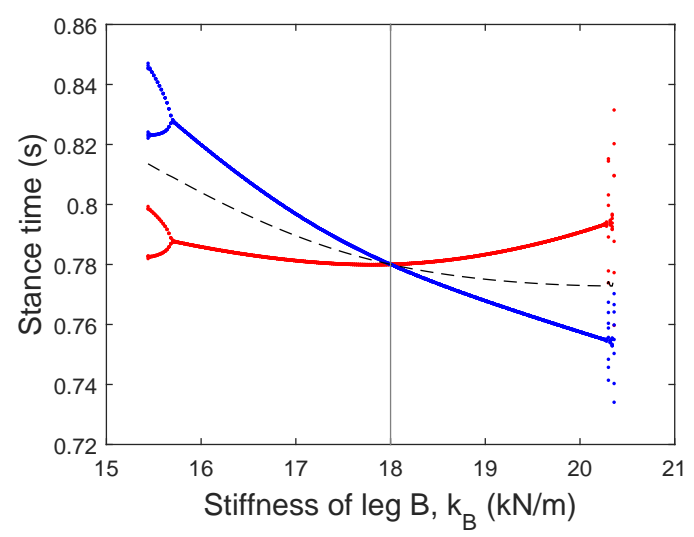

(b)

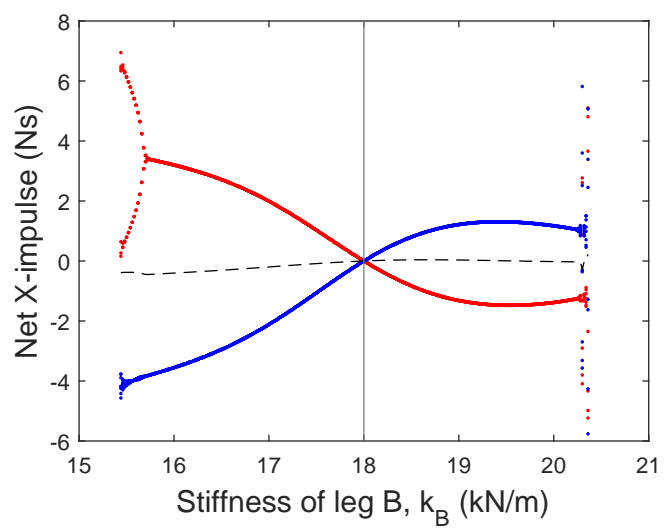

(d)

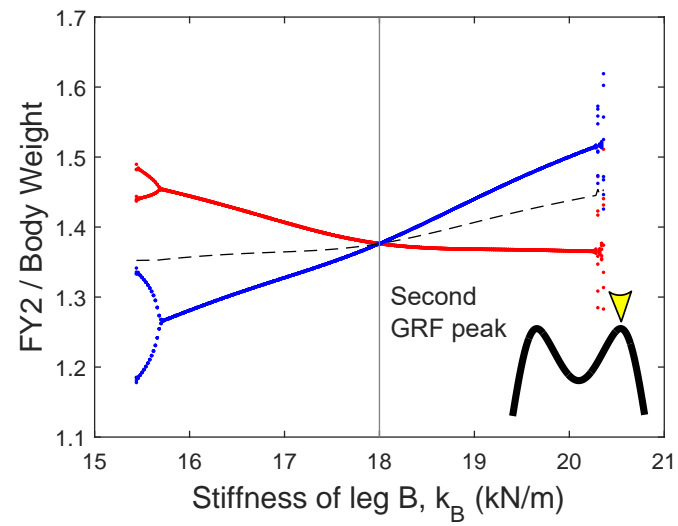

(f)

Figure 5-11: Effect of changing the effective leg stiffness of one leg, $k_{B}$, on the gait characteristics of the SLPW model. The blue points represents gait characteristics while the unaffected leg is the support leg, the red points indicates that the modified leg is the support. The other leg remains at $k_{A}=18 \mathrm{kN} / \mathrm{m}$. 


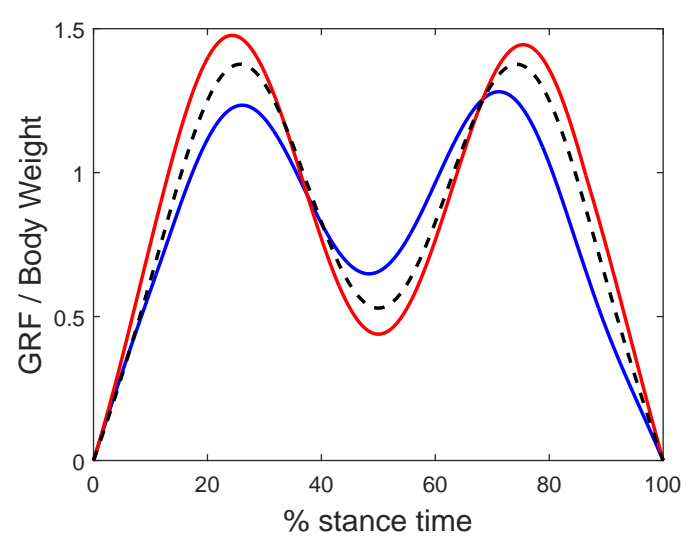

(a) $k_{B}=16 \mathrm{kN} / \mathrm{m}$

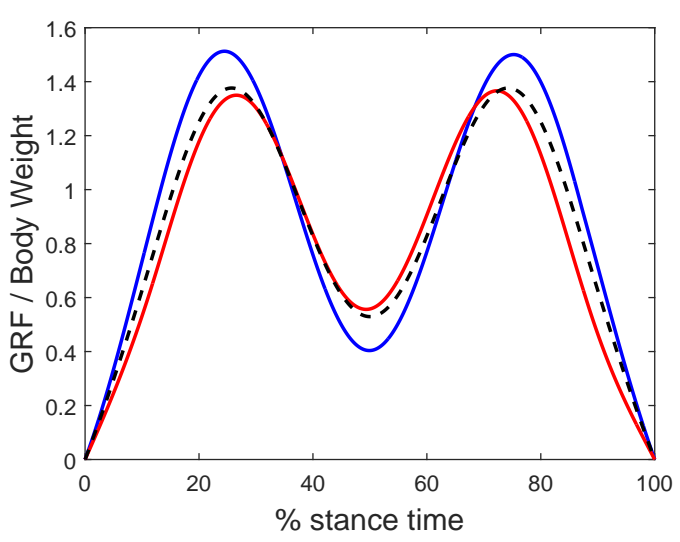

(b) $k_{B}=20 \mathrm{kN} / \mathrm{m}$

Figure 5-12: Vertical ground reaction force (GRF) profile for the SLIP walking model with the stiffness of leg $B, k_{B}$, being equal to (a) $16 \mathrm{kN} / \mathrm{m}$ and (b) $20 \mathrm{kN} / \mathrm{m}$ (shown by the red line), while the other leg touch-down angle, $k_{A}$, remains at $18 \mathrm{kN} / \mathrm{m}$ (shown by the blue line). The GRF plot for a symmetric model with $k$ being $18 \mathrm{kN} / \mathrm{m}$ for both legs is plotted as the dashed black line. Both maximum peaks are larger for the compliant limb.

\subsubsection{Asymmetric roller radius, $R$}

The roller radius or roll-over shape [47] represents the kinematic trajectory of the hip during stance phase and provides better estimations than the point-foot inverted pendulum [147]. The roll-over shape that most people conform to is a radius of approximately $30 \%$ of the leg length $[94,2]$. People will adapt their ankle kinematics to maintain this roll-over shape [145]. If a person is impaired due to injury, neuromuscular problems or from an improperly aligned prosthesis/orthosis, this roll-over shape may not be optimal.

To investigate the effects of asymmetry in roll-over shape, this model simulates walking with a variation of one leg's rocker radius, $R_{B}$. A larger radius of curvature produces a flatter foot, which increases the stance time and inter-leg angle at heelstrike for that leg, while decreasing the value for the contralateral limb (Figures 5-13a \& 5-13b).

From Figure 5-13d it can be seen that a flatter foot contributes to propulsion and 
a point foot contributes to braking during steady state walking. A small variation is seen in the contributions to the vertical GRF peaks in Figures 5-13e and 5-13f. Full vertical GRF plots are shown for $R_{B}$ equal to $0.26 \mathrm{~m}$ and $0.33 \mathrm{~m}$ in Figures 5-14a and 5-14b respectively. The first peak, during the first half of the stance phase has a larger variation with the flatter foot having a larger vertical GRF.

\subsection{Discussion of results}

To gain understanding into the dynamics of asymmetric gait, a new model is proposed: a spring-loaded passive walking model with rolling feet. This model produced symmetric, stable walking with kinematics and kinetics similar to human gait. Variations of a single leg's mass ratio, $\mu_{B}$, length ratio, $\beta_{B}$, leg stiffness, $k_{B}$, and rocker radius, $R_{B}$, were simulated and gait characteristics for stable walking conditions were found that exhibited asymmetric gait patterns.

A prosthetic foot has a smaller push-off force, longer step length, longer swing time and a shorter stance time than the intact limb [92]. According to research from Zelik et al. [151], a softer spring for a prosthetic leads to greater energy storage, energy return and prosthetic limb COM push-off work. Amputees experience a larger FY1 peak on their intact limb compared to their prosthetic limb and a greater chance of osteoarthritis for the sound limb $[27,103,114]$. This model suggests that a larger force for the sound limb may be due to a stiffer contralateral prosthetic limb. A larger leg stiffness of leg $B, k_{B}$, induced a slower walking speed, with a shorter inter-leg angle and longer stance time for the stiffer limb. The maximum peaks for the ground reaction forces (GRFs) shows that a stiffer limb reduced maximum forces for that limb while increasing the forces for the unaffected, more compliant, contralateral limb. Reducing a limb stiffness from $18 \mathrm{kN} / \mathrm{m}$ to $16 \mathrm{kN} / \mathrm{m}$ increased the first and second maximum vGRF peaks by $7.3 \%$ and $4.9 \%$ respectively, while decreasing the vGRF peaks for the stiffer contralateral limb by $10.3 \%$ and $6.9 \%$ respectively. Previous 


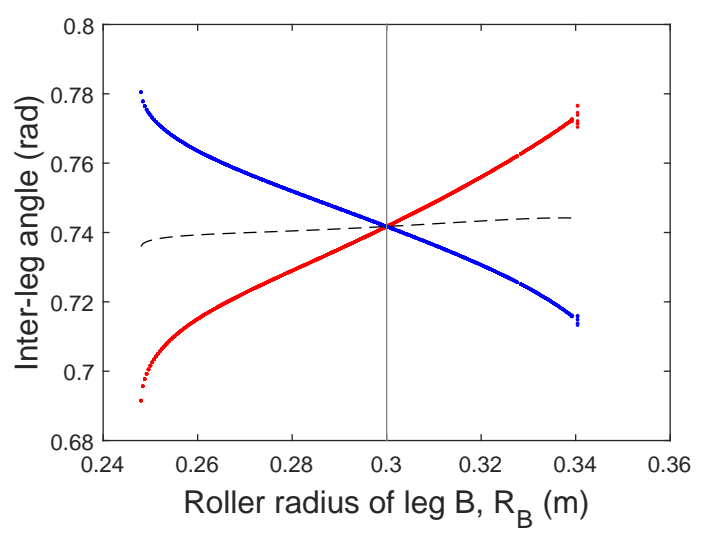

(a)

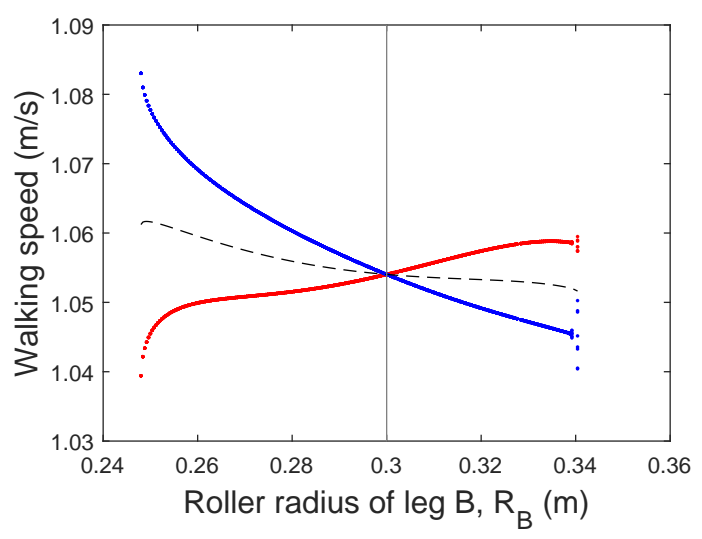

(c)

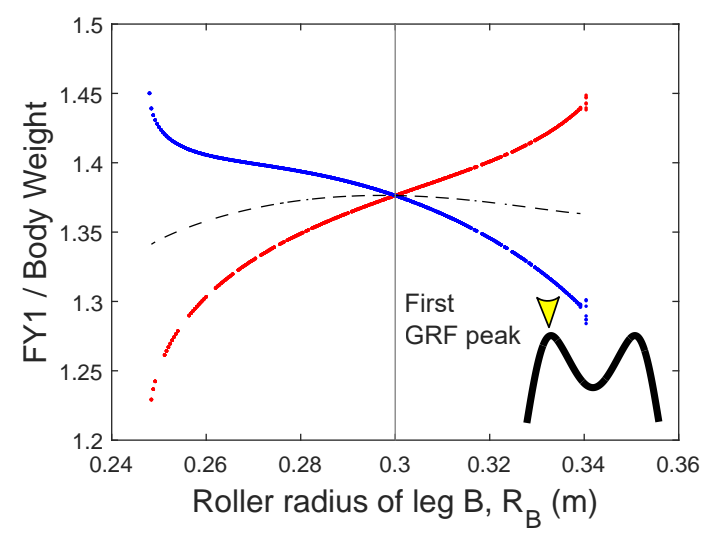

(e)

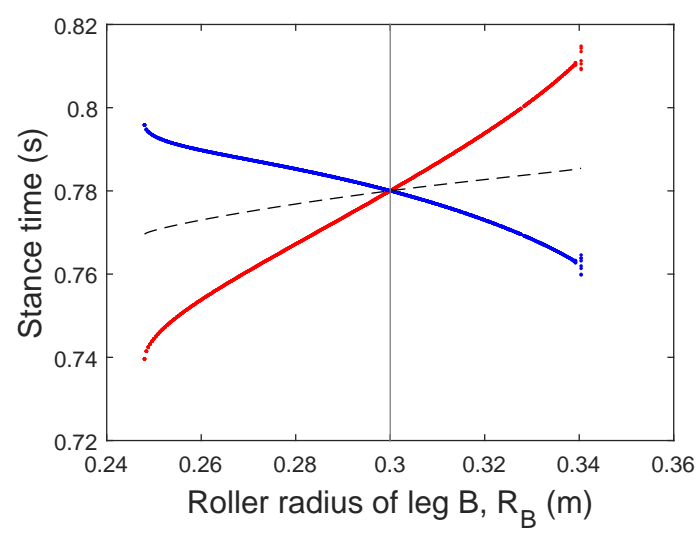

(b)

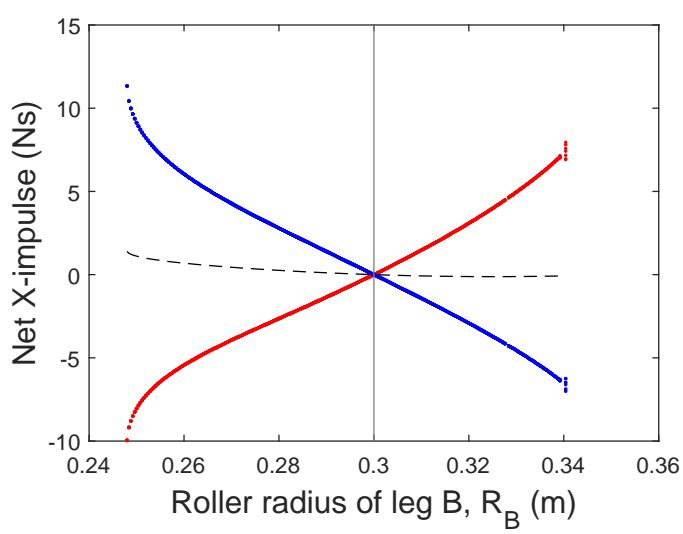

(d)

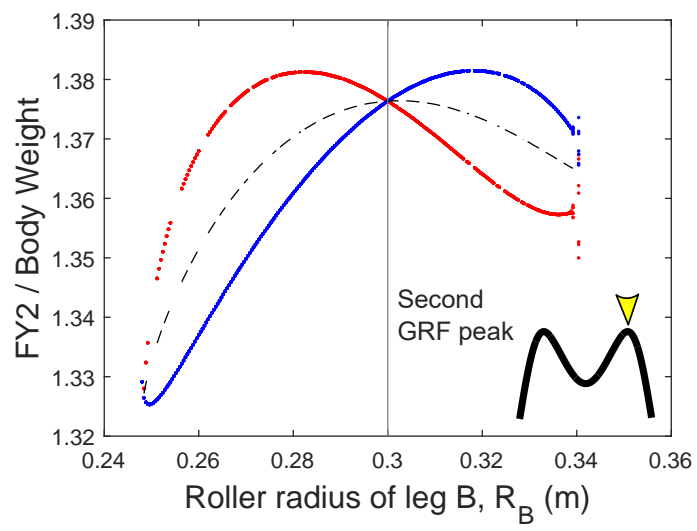

(f)

Figure 5-13: Effect of changing the rocker radius $R_{B}$ of one leg on the gait characteristics of the SLPW model. The blue points represents gait characteristics while the unaffected leg is the support leg, the red points indicates that the modified leg is the support. The other leg remains at $R_{A}=30 \%$ leg length. 


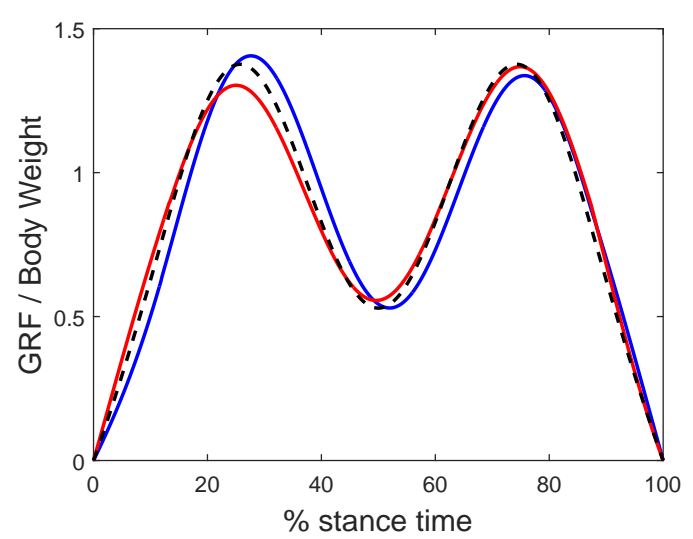

(a) $R_{B}=0.26 \mathrm{~m}$

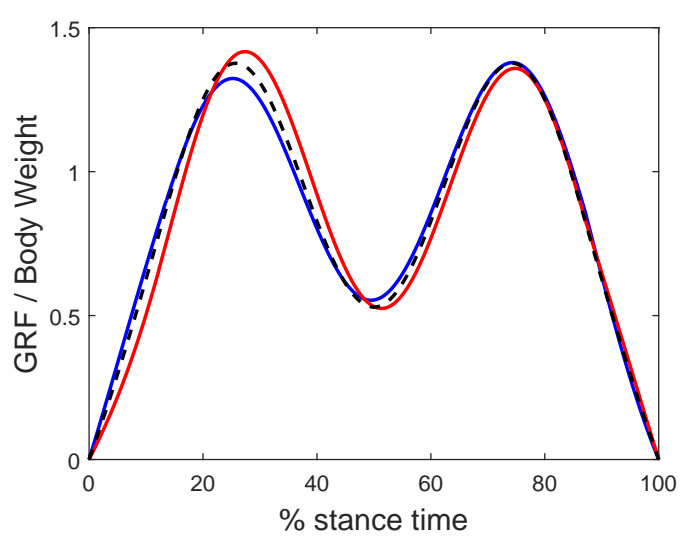

(b) $R_{B}=0.33 \mathrm{~m}$

Figure 5-14: Vertical ground reaction force (GRF) profile for the SLIP walking model with the stiffness of leg $B, R_{B}$, being equal to (a) $0.26 \mathrm{~m}$ and (b) $0.33 \mathrm{~m}$ (shown by the red line), while the other leg touch-down angle, $R_{A}$, remains at $0.30 \mathrm{~m}$ (shown by the blue line). The GRF plot for a symmetric model with $R$ being $0.30 \mathrm{~m}$ for both legs is plotted as the dashed black line.

research suggests that the ankle provides a larger impulse for the trailing leg than the remaining body during the launching phase [82]. This behaviour was not simulated using the SLIP model as the legs are massless.

The mass and centre-of-mass position of a single limb were varied to investigate how the inertial properties of the limb affect gait. Variation of the mass ratio of the leg, $\mu$, retained symmetry however the walking speed increases with increasing $\mu_{B}$. The position of leg COM may also be represented by the leg length ratio, $\beta_{B}$. From alterations of $\beta_{B}$ the stance time remains the mostly unchanged. However, the step length and walking speed increases for a lower limb mass, while decreasing the step length for the unchanged mass. A study by Hekmatfard et al. [50] investigated the effect of changing the prosthetic COM and found that there were no significant changes for increasing the weight of the prosthetic, however adding weight to the ankle - and lowering the position of prosthetic COM location - induced higher stepping speeds and step lengths for the prosthetic limb than the intact limb. This is the same results for the SLPW model investigated in this section. These results were not replicated with the rigid legged PW walker in Section 3.4.1. 
The SLPW model is not robust enough to handle large asymmetries. Prosthetic feet are around $\mu$ and $\beta$. Importantly, the gait dynamics of one leg will have an effect not just on an altered limb but also on the gait characteristics of the contralateral unaffected limb. 


\section{Chapter 6}

\section{Conclusions}

\subsection{Motivation}

Amputees suffer from high rates of residual limb and back pain [28] and increased risk of falling that has an effect on their quality of living [70, 96]. A person may want to achieve symmetric walking to walk more cosmetically, expend less energy or have natural joint loads to reduce further injury. Unilateral lower-limb amputees demonstrate significantly increased rates of osteoarthritis in their sound knee [104, 114] and individuals with transfemoral (above-knee) amputations are three times more likely to develop hip osteoarthritis than transtibial (below-knee) amputees [71].

Prosthetics or orthoses are devices used to rehabilitate a person's gait and ultimately improve their quality of life. The aim of this thesis is to use dynamic walking models to understand how walking characteristics are affected by gait asymmetries and to provide insight into rehabilitation to improve the understanding of prosthetic and orthosis design. To accomplish this, passive walking and spring-mass models were used without active controllers. These have been shown to exhibit human-like gait behaviour and have been used as models before to describe locomotion biomechanics [26, 81, 152, 127]. 


\subsection{Discussion of Results}

In this thesis, dynamic walking models with no controllers or actuators are utilised to gain an understanding of the effects of asymmetric masses, leg stiffnesses and roll-over radii on the passive dynamics of gait. The passive walker $(\mathrm{PW})$, springloaded inverted pendulum (SLIP) and spring-loaded passive walker (SLPW) provided insights into the stability and symmetry of bipedal walking dynamics with a range of asymmetric parameters. Gait characteristics such as walking speed, step length and ground reaction forces (GRFs) were evaluated to provide insight into how locomotion is affected by altered parameters.

A prosthesis usually weighs $30-40 \%$ of the weight of an intact limb [92] and this mass imbalance has an effect on the dynamics of walking $[131,55]$. However, matching a prosthesis' inertial properties to the intact limb produces greater asymmetries and higher energy costs [92, 132], demonstrating that mass imbalance is not the only factor that affects an amputee's gait. Research from Selles et al. [122] shows that amputees will adapt to mass perturbations by maintaining their joint kinematics (joint angles), rather than their kinetics (joint torques). The research in this thesis outlines how the dynamics of gait is affected by the mass and the position of the centre of mass (COM) of the leg. The PW and SLPW models predict that asymmetric gait characteristics are more sensitive to position of leg COM ratio, $\beta$, rather than mass ratio, $\mu$. Empirical results suggest that lowering the position of prosthesis COM by adding a mass to the ankle produces a more noticeable effect than adding mass to the thigh $[78,50,132]$. Significant asymmetries for altered $\beta_{B}$ were found only with the inclusion of modelling springy legs in the SLPW model, which had the effect of simulating a correct human-like GRF pattern. The SLPW also predicts a shorter stance time for a prosthetic leg which is seen in amputees $[4,59,91,25]$ and predicts that a higher COM position of a residual limb contributes to larger forces in the sound limb.

The SLIP model has a single concentrated mass at the hip and the touch-down 
angle of the leg at the beginning of a step is defined by $\alpha$. Using the SLIP it is concluded that an asymmetric touch-down angle or effective leg stiffness does not induce asymmetries in stance time but causes an alteration in inter-leg angle and step speed symmetry. This is in alignment with empirical evidence that there is a significant relationship between step length and walking velocity [98]. Although the stance time remains symmetric, the variation with respect to $\alpha_{B}$ is noticeable, meaning that altering the dynamics of one limb will also alter the effects of the healthy contralateral limb by an appreciable amount.

The SLIP model predicts that a stiffer leg will have higher vGRF forces, while the SLPW model predicts the opposite; a more compliant leg will have larger vGRFs. Discrepancies are due to the propulsion of the swing leg as the SLPW simulates the leg masses to predict the ballistics of the swing leg. Previous research suggest that the ankle propulsion provides a larger impulse to the swing leg than the remaining body [82], which is not simulated in the SLIP model. Importantly, an altered leg stiffness has a noticeable effect on the opposite contralateral limb, which could suggest why amputees have larger rates of osteoarthritis on their sound limb [114]. Experimental results suggest that the sound limb is exposed to higher vertical reaction forces, with the prosthetic foot design having a direct effect on the reaction forces [133].

By studying the dynamics of the SLPW model, it can be seen that a stiffer leg results in smaller touch-down angles and peak vGRFs for that limb, while increasing the vGRFs and decreasing the stance time of the more compliant contralateral limb. An amputee exhibits less push-off force from their prosthesis [78] with a longer stump length increasing propulsion from the residual limb [98]. The impulse from the prosthetic leg may be improved with altered touch-down angle [118] or a lower spring stiffness [151].

By aligning the centre of pressure (COP) of the foot along a coordinate system aligned with the stance leg, it can be seen that people walk on a rocker roller radius of about $30 \%$ the leg length. This roll-over shape does not change with variation 
of walking speeds [47], ground inclines [48], heel height [45] or added weights to the torso [46]. The design of prosthetic limbs and ankle-foot orthoses determine the COP progression during walking. The effect of asymmetric roller radii can be seen in all three of the models used, with the walking speed increasing for the foot with the larger roller radius, $R_{B}$. The PW and SLPW, two models with leg masses, had the most noticeable asymmetries; however the larger $R_{B}$ resulted in a shorter stance time for the PW model and a longer stance time for the SLPW.

The discrepancies between theoretical models and experimental findings may be due to poor methodologic quality of the experiments as well as the limited predictive value of existing models [121]. Although there are limitations to these models, this work may be used to explore methods for improving prostheses and gain insight into the reasons amputees expend more energy. This work can be used to gain investigate how gait asymmetries are affected by dynamic effects rather that physiological or neurological problems and ultimately design clinical experiments to help design future prosthetics, orthotics or exoskeletons intended for gait rehabilitation.

\subsection{Future work}

In this thesis, gait characteristics are evaluated at steady and stable walking conditions. Delving further into these models, it may be worth investigating the robustness of certain gait parameters and how quantify the amount of time needed to retain steady, stable walking after a perturbation [37]. Basin of attraction computations would quantify the ability for the model to recover under random perturbations such as slipping or uneven ground [120]. Further research may also include investigations into asymmetry at a range of walking speeds. Research shows that walking asymmetries due to prosthesis are reduced at higher walking speeds [25, 103]

Naturally there is space for these simple models to gain complexity by adding active controllers, an upper torso for balance [34] or knee and ankle joints [139]. Ex- 
tending these models into 3D may be used to investigate lateral balance and stability [53]. The swing leg mechanics of the SLPW model can also be improved by adding compliance to the swinging leg instead of a rigid link [134]. The virtual pivot point (VPP) methodology for control (as discussed in Section 2.2.6) may provide a simple control method for biped models. Sharbafi and Seyfarth [126] uses a spring-mass model with a controller for the swing leg and VPP control to mimic gait dynamics. This VPP is seen in humans and other animals as a phenomenon during walking, it would be interesting to see if this is appropriately used by persons walking with impairments to maintain stable gait.

A linear spring stiffness has been assumed to mimic the dynamics of gait [81], however it may be worth investigating nonlinear spring stiffnesses at different walking speeds or the effect of a damping mechanism. The ankle mechanics can be described with a 'catch' mechanism [82], with the ankle rapidly unloading before the swing phase. The timing of ankle push-off may be incorporated into devices such as prosthetic feet or exoskeletons [117] to help people walk with minimal energy costs and reduce excessive joint loads.

This research is intended to provide an understanding to help design further experiments. 3D musculoskeletal models simulate muscles, ligaments and bone structures for biomechanical analysis $[21,19,128,13,86]$. Joint forces, moments and the muscular power required to perform certain motions are estimated from gait data and body positions. These multibody systems can also be used to explore gait with changing musculoskeletal geometry and other model parameters. It would be interesting to compare the results of these passive models to these complex musculoskeletal simulations with embedded control. 


\section{Appendix A}

\section{Calculating the Ground Reaction}

\section{Forces}

In between each collision, there are no external forces on the system, therefore angular momentum is conserved and the line of action points towards the COM. This method is similar to Whittington and Thelen [147]'s method of finding the GRFs, except with the inclusion of leg masses if being used for the SLPW model and a schematic shown in Figure A-1. Thus, the ground force vector for each limb is given by:

$$
\theta_{l a}=\tan ^{-1}\left(\frac{X_{\mathrm{COM}}-s}{Y_{\mathrm{COM}}}\right)
$$

The component of GRF vector acting along the spring (and along the $y$-axis) is

$$
F_{y}=k_{1} r_{1}
$$

Knowing the spring force $F_{y}$ and the force vector direction $\theta_{l a}$, the force perpendicular to the spring, $F_{x}$ is

$$
F_{x}=-F_{y} \tan \left(\theta_{1}-\theta_{l a}\right)
$$


The resultant force is then $F_{R}=\sqrt{F_{x}{ }^{2}+F_{y}{ }^{2}}$ and acts from the COP to the COM at an angle of $\theta_{l a}$. The vertical and horizontal anterior-posterior GRFs can now be determined:

$$
\begin{aligned}
& F_{X}=F_{R} \sin \left(\theta_{l a}\right) \\
& F_{Y}=F_{R} \cos \left(\theta_{l a}\right)
\end{aligned}
$$

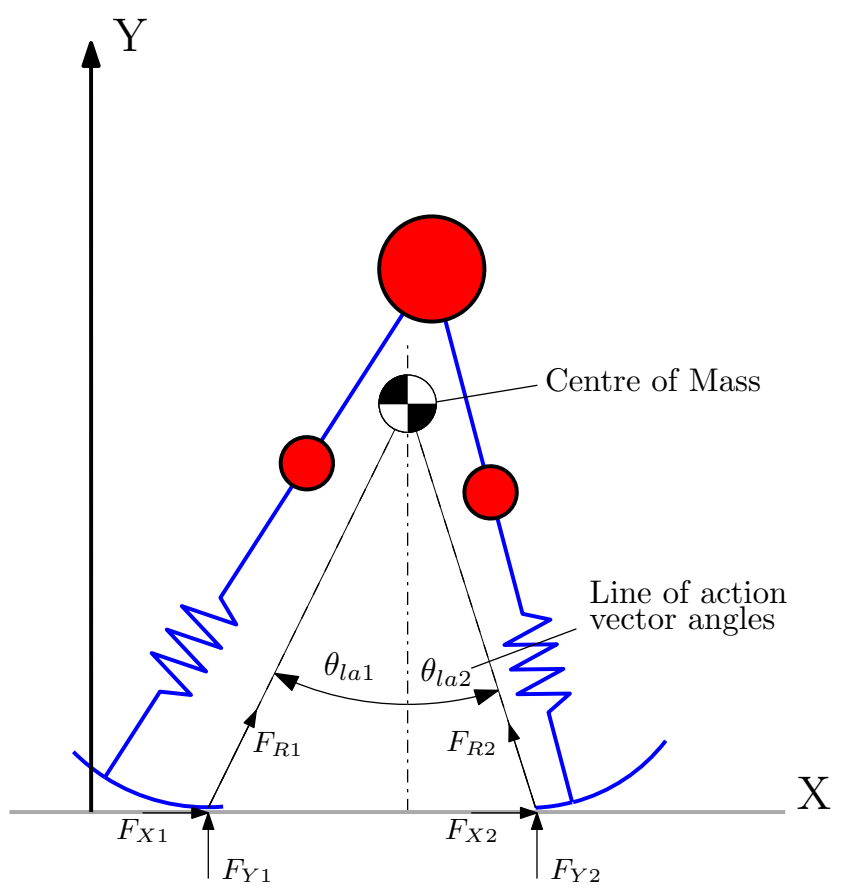

(a) The line of action $\theta_{l a}$ for each limb points from the COP to the COM.

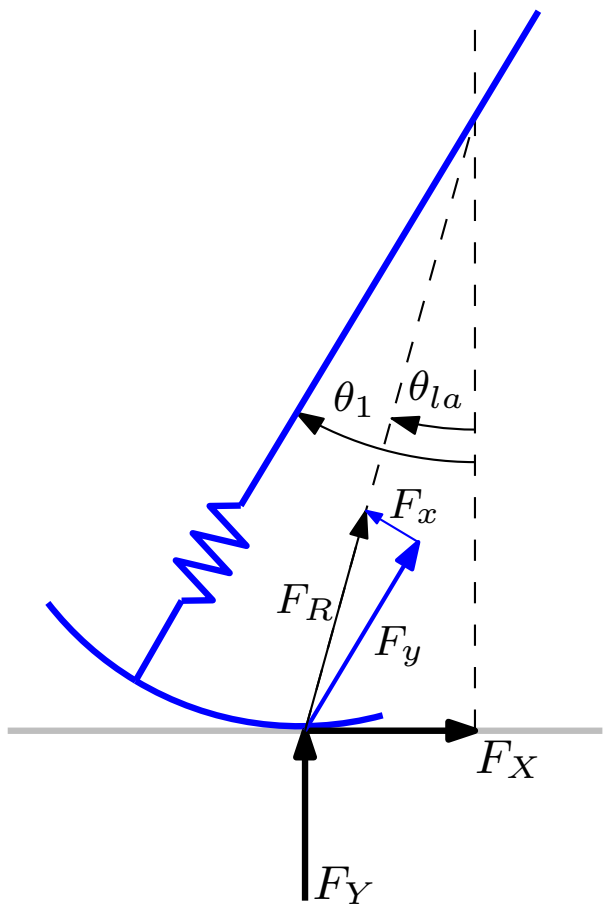

(b) The Ground Reaction Force (GRF) vectors $F_{X}$ and $F_{Y}$ for each limb are calculated from the spring force $F_{y}$ and the angle of line of action vector $\theta_{l a}$.

Figure A-1: Calculating vertical and horizontal GRFs knowing the location of COM, COP and spring extensions. 


\title{
Glossary
}

\author{
Contralateral limb Opposite limb \\ Dorsiflexion Movement of the ankle so that toes move towards the shin \\ Force platform An instrument that measures ground reaction forces \\ (GRFs) \\ Inter-leg angle The angle between the legs, $\theta_{2}$ \\ Kinematics The study of motion of objects \\ Kinetics The study of forces that cause motion \\ MATLAB ode45 A numerical integration solver that uses the \\ Runge-Kutta method \\ Metabolic energy Biological energy spent \\ Residual limb Limb that has suffered an amputation \\ Roll-over shape Effective rocker shape that the lower-limb system conforms \\ to during stance phase \\ Stance phase Period of time while leg is in contact with the ground \\ Step Heel-strike from opposite leg heel-strike \\ Stride Full walking cycle from heel-strike to same leg heel-strike \\ Swing phase Period of time while leg is in the air \\ Touch-down angle Angle of the swing leg at the floor collision, denoted by $\alpha$ \\ or $\phi$ \\ Transfemoral Above knee \\ Transtibial Below knee
}




\section{Bibliography}

[1] P. G. Adamczyk and A. D. Kuo. Redirection of center-of-mass velocity during the step-to-step transition of human walking. The Journal of experimental biology, 212(Pt 16):2668-78, aug 2009. ISSN 0022-0949. doi: 10.1242/jeb. 027581. URL https://www.ncbi.nlm.nih.gov/pubmed/19648412.

[2] P. G. Adamczyk, S. H. Collins, and A. D. Kuo. The advantages of a rolling foot in human walking. The Journal of experimental biology, 209(Pt 20):3953-63, oct 2006. ISSN 0022-0949. doi: 10.1242/jeb.02455. URL http://www.ncbi. nlm.nih.gov/pubmed/17023589.

[3] M. M. Ankaral, S. Sefati, M. S. Madhav, A. Long, A. J. Bastian, and N. J. Cowan. Walking dynamics are symmetric (enough). Journal of The Royal Society Interface, 12(108):20150209, 2015. ISSN 1742-5689. doi: 10.1098/rsif. 2015.0209. URL http://rsif.royalsocietypublishing.org/lookup/doi/ 10.1098/rsif. 2015.0209.

[4] P. A. Baker and S. R. Hewison. Gait recovery pattern of unilateral lower limb amputees during rehabilitation. Prosthetics and orthotics international, 14:8084, 1990. ISSN 0309-3646. doi: 10.3109/03093649009080327.

[5] J. E. A. Bertram and A. Ruina. Multiple walking speed-frequency relations are predicted by constrained optimization. Journal of Theoretical Biology, 209(4): 445-453, 2001. ISSN 00225193. doi: 10.1006/jtbi.2001.2279.

[6] J. M. Brockway. Derivation of formulae used to calculate energy expenditure in man. Hum Nutr Clin Nutr, 41(6):463-471, 1987. ISSN 0263-8290. doi: 10.1016/j.pcad.2015.11.006.

[7] S. M. Bruijn, O. G. Meijer, P. J. Beek, and J. H. van Dieen. Assessing the stability of human locomotion: a review of current measures. Journal of The Royal Society Interface, 10(83):20120999-20120999, 2013. ISSN 1742-5689. doi: 10.1098/rsif.2012.0999. URL http://rsif.royalsocietypublishing. org/cgi/doi/10.1098/rsif.2012.0999.

[8] M. J. Burke, V. Roman, and V. Wright. Bone and joint changes in lower limb amputees. Annals of the Rheumatic Diseases, 37(3):252-254, 1978. ISSN 00034967. doi: 10.1136/ard.37.3.252. 
[9] G. A. Cavagna and R. Margaria. Mechanics of walking. J Appl Physiol, 21(1): 271-278, 1966. ISSN 0021-8987. URL https://www.physiology.org/doi/ abs/10.1152/jappl.1966.21.1.271.

[10] G. A. Cavagna, F. P. Saibene, and R. Margaria. External work in walking. Journal of applied physiology (Bethesda, Md. : 1985), 18(3):1-9, 1963. ISSN 0021-8987.

[11] G. A. Cavagna, N. C. Heglund, and C. R. Taylor. Mechanical work in terrestrial locomotion: Two basic mechanisms for minimizing energy expenditure. American Journal of Physiology, 233:R243-R261, 1977. ISSN 0002-9513. URL http://ajpregu.physiology.org/content/233/5/R243. abstract.

[12] W. G. Charles, P. Mahmoodi, R. S. Ransing, I. Sazonov, and M. I. Friswell. Comparison of point foot, collisional and smooth rolling contact models on the bifurcations and stability of bipedal walking. European Journal of Computational Mechanics, 7179(June):1-21, 2016. ISSN 19585829. doi: 10.1080/ 17797179.2016.1191122. URL http://dx.doi.org/10.1080/17797179.2016. 1191122.

[13] M. Christophy, N. A. F. Senan, J. C. Lotz, and O. M. O'Reilly. A Musculoskeletal model for the lumbar spine. Biomechanics and Modeling in Mechanobiology, 11(1-2):19-34, 2012. ISSN 16177959. doi: 10.1007/s10237-011-0290-6.

[14] T. Chyou, G. F. Liddell, and M. G. Paulin. An upper-body can improve the stability and efficiency of passive dynamic walking. Journal of Theoretical Biology, 285(1):126-135, 2011. ISSN 00225193. doi: 10.1016/j.jtbi.2011.06.032. URL http://dx.doi.org/10.1016/j.jtbi.2011.06.032.

[15] S. Collins, A. Ruina, R. Tedrake, and M. Wisse. Efficient bipedal robots based on passive-dynamic walkers. Science, 307(5712):1082-5, feb 2005. ISSN 10959203. doi: 10.1126/science.1107799. URL http://www.ncbi.nlm.nih.gov/ pubmed/15718465.

[16] S. H. Collins. A Three-Dimensional Passive-Dynamic Walking Robot with Two Legs and Knees. The International Journal of Robotics Research, 20(7):607615, jul 2001. ISSN 0278-3649. doi: 10.1177/02783640122067561. URL http: //ijr.sagepub.com/cgi/doi/10.1177/02783640122067561.

[17] S. H. Collins. Dynamic walking principles applied to human gait. PhD thesis, The University of Michigan, 2008.

[18] C. Curtze, A. L. Hof, H. G. van Keeken, J. P. K. Halbertsma, K. Postema, and B. Otten. Comparative roll-over analysis of prosthetic feet. Journal of biomechanics, 42(11):1746-53, aug 2009. ISSN 1873-2380. doi: 10.1016/j.jbiomech. 2009.04.009. URL http://www.ncbi.nlm.nih.gov/pubmed/19446814. 
[19] M. Damsgaard, J. Rasmussen, S. T. Christensen, E. Surma, and M. de Zee. Analysis of musculoskeletal systems in the AnyBody Modeling System. Simulation Modelling Practice and Theory, 14(8):11001111, nov 2006. ISSN 1569-190X. doi: 10.1016/J.SIMPAT.2006. 09.001. URL https://www.sciencedirect.com/science/article/pii/ S1569190X06000554?via\{\%\}3Dihub.

[20] P. de Leva. Adjustments to Zatsiorsky-Seluyanov's segment intertia parameters. Journal of Biomechanics, 29(9):1223-1230, 1996. ISSN 0021-9290. doi: 10.1016/ 0021-9290(95)00178-6.

[21] S. L. Delp and J. P. Loan. A graphics-based software system to develop and analyze models of musculoskeletal structures. Computers in Biology and Medicine, 25(1):21-34, jan 1995. ISSN 00104825. doi: 10.1016/0010-4825(95) 98882-E. URL https://www.sciencedirect.com/science/article/pii/ 001048259598882E.

[22] J. M. Donelan, R. Kram, and A. D. Kuo. Mechanical and metabolic determinants of the preferred step width in human walking. Proceedings of the Royal Society B: Biological Sciences, 268(1480):1985-1992, 2001. ISSN 0962-8452. doi: 10.1098/rspb.2001.1761. URL http://rspb.royalsocietypublishing. org/cgi/doi/10.1098/rspb.2001.1761.

[23] J. M. Donelan, R. Kram, and A. D. Kuo. Mechanical work for step-to-step transitions is a major determinant of the metabolic cost of human walking. The Journal of experimental biology, 205(Pt 23):3717-3727, 2002. ISSN 0022-0949.

[24] J. M. Donelan, R. Kram, and A. D. Kuo. Simultaneous positive and negative external mechanical work in human walking. Journal of Biomechanics, 35(1):117-124, jan 2002. ISSN 00219290. doi: 10.1016/S0021-9290(01) 00169-5. URL http://www.sciencedirect.com/science/article/pii/ S0021929001001695?via\{\%\}3Dihub.

[25] S. F. Donker and P. J. Beek. Interlimb coordination in prosthetic walking: effects of asymmetry and walking velocity. Acta psychologica, 110:265-288, 2002. ISSN 00016918. doi: 10.1016/S0001-6918(02)00037-9.

[26] K. Endo, D. Paluska, and H. Herr. A quasi-passive model of human leg function in level-ground walking. IEEE International Conference on Intelligent Robots and Systems, pages 4935-4939, 2006. doi: 10.1109/IROS.2006.282454.

[27] J. R. Engsberg, A. G. Lee, J. L. Patterson, and J. A. Harder. External loading comparisons between able-bodied and below-knee-amputee children during walking. Archives of physical medicine and rehabilitation, 72(9):657-661, 1991. ISSN 00039993. doi: 10.5555/URI:PII:0003999391902092. 
[28] P. L. Ephraim, S. T. Wegener, E. J. MacKenzie, T. R. Dillingham, and L. E. Pezzin. Phantom pain, residual limb pain, and back pain in amputees: Results of a national survey, 2005. ISSN 00039993.

[29] A. Erdemir, S. McLean, W. Herzog, and A. J. van den Bogert. Model-based estimation of muscle forces exerted during movements. Clinical Biomechanics, 22(2):131-154, 2007. ISSN 02680033. doi: 10.1016/j.clinbiomech.2006.09.005.

[30] A. Esquenazi. Gait analysis in lower-limb amputation and prosthetic rehabilitation, feb 2014. ISSN 10479651. URL https://www.sciencedirect.com/ science/article/pii/S1047965113000739?via=ihub.

[31] D. J. Farris and G. S. Sawicki. The mechanics and energetics of human walking and running: a joint level perspective. Journal of the Royal Society, Interface / the Royal Society, 9(66):110-8, 2012. ISSN 1742-5662. doi: 10.1098/rsif.2011.0182. URL http://rsif.royalsocietypublishing.org/ content/early/2011/05/20/rsif .2011.0182. short.

[32] N. P. Fey, G. K. Klute, and R. R. Neptune. The influence of energy storage and return foot stiffness on walking mechanics and muscle activity in belowknee amputees. Clinical biomechanics (Bristol, Avon), 26(10):1025-32, dec 2011. ISSN 1879-1271. doi: 10.1016/j.clinbiomech.2011.06.007. URL http: //www.ncbi.nlm.nih.gov/pubmed/21777999.

[33] N. P. Fey, G. K. Klute, and R. R. Neptune. Altering prosthetic foot stiffness influences foot and muscle function during below-knee amputee walking: a modeling and simulation analysis. Journal of biomechanics, 46(4):63744, feb 2013. ISSN 1873-2380. doi: 10.1016/j.jbiomech.2012.11.051. URL http://www.ncbi.nlm.nih.gov/pubmed/23312827.

[34] L. L. Flynn, R. Jafari, and R. Mukherjee. Active Synthetic-Wheel Biped With Torso. IEEE Transactions on Robotics, 26(5):816-826, oct 2010. ISSN 15523098. doi: 10.1109/TRO.2010.2061272. URL http://ieeexplore.ieee.org/ lpdocs/epic03/wrapper .htm?arnumber $=5559518$.

[35] T. Fukunaga, K. Kubo, Y. Kawakami, S. Fukashiro, H. Kanehisa, and C. N. Maganaris. In vivo behaviour of human muscle tendon during walking. Proc. R. Soc, 268(1464)(February 2000):229-233, 2001. doi: 10.1098/rspb.2000.1361.

[36] R. S. Gailey, M. S. Nash, T. A. Atchley, R. M. Zilmer, G. R. Moline-Little, N. Morris-Cresswell, and L. I. Siebert. The effects of prosthesis mass on metabolic cost of ambulation in non-vascular trans-tibial amputees. Prosthetics and orthotics international, 21(1):9-16, 1997. ISSN 0309-3646. doi: 10.3109/03093649709164525.

[37] M. Garcia, A. Chatterjee, A. Ruina, and M. Coleman. The simplest walking model: stability, complexity, and scaling. Journal of biomechanical engineering, 
120(2):281-8, apr 1998. ISSN 0148-0731. URL http://www.ncbi.nlm.nih. gov/pubmed/10412391.

[38] S. A. Gard, S. C. Miff, and A. D. Kuo. Comparison of kinematic and kinetic methods for computing the vertical motion of the body center of mass during walking. Human Movement Science, 22(6):597-610, 2004. ISSN 01679457. doi: 10.1016/j.humov.2003.11.002. URL http://linkinghub.elsevier.com/ retrieve/pii/S016794570300085X.

[39] H. Geyer, A. Seyfarth, and R. Blickhan. Compliant leg behaviour explains basic dynamics of walking and running. Proceedings. Biological sciences / The Royal Society, 273(1603):2861-7, nov 2006. ISSN 0962-8452. doi: 10.1098/rspb.2006. 3637.

[40] A. Goswami, B. Thuilot, and B. Espiau. Compass-like biped robot Part I : Stability and bifurcation of passive gaits. Technical Report RR-2996, 1996.

[41] A. Goswami, B. Thuilot, and B. Espiau. A Study of the Passive Gait of a Compass-Like Biped Robot: Symmetry and Chaos. The International Journal of Robotics Research, 17(12):1282-1301, 1998. ISSN 0278-3649. doi: 10.1177/ 027836499801701202.

[42] R. D. Gregg, Y. Dhaher, and K. M. Lynch. Functional asymmetry in a five-link 3D bipedal walker. Proceedings of the Annual International Conference of the IEEE Engineering in Medicine and Biology Society, EMBS, pages 7820-7823, 2011. ISSN 1557170X. doi: 10.1109/IEMBS.2011.6091927.

[43] R. D. Gregg, A. Degani, Y. Dhaher, and K. M. Lynch. The Basic Mechanics of Bipedal Walking Lead to Asymmetric Behavior. Changes, 29:997-1003, 2012. ISSN 15378276. doi: 10.1016/j.biotechadv.2011.08.021.Secreted.

[44] R. D. Gregg, Y. Y. Dhaher, A. Degani, and K. M. Lynch. On the mechanics of functional asymmetry in bipedal walking. IEEE Transactions on Biomedical Engineering, 59(5):1310-1318, 2012. ISSN 00189294. doi: 10.1109/TBME.2012. 2186808.

[45] A. H. Hansen and D. S. Childress. Effects of shoe heel height on biologic rollover characteristics during walking. Journal of rehabilitation research and development, 41:547-554, 2004. ISSN 0748-7711. doi: 10.1682/JRRD.2003.06. 0098.

[46] A. H. Hansen and D. S. Childress. Effects of adding weight to the torso on rollover characteristics of walking. Journal of rehabilitation research and development, 42(3):381-390, 2005. ISSN 0748-7711. doi: 10.1682/JRRD.2004.04.0048.

[47] A. H. Hansen, D. S. Childress, and E. H. Knox. Roll-over shapes of human locomotor systems: Effects of walking speed. Clinical Biomechanics, 19(4): 407-414, may 2004. ISSN 02680033. doi: 10.1016/j.clinbiomech.2003.12.001. URL http://www.ncbi.nlm.nih.gov/pubmed/15109762. 
[48] A. H. Hansen, D. S. Childress, and S. C. Miff. Roll-over characteristics of human walking on inclined surfaces. Human movement science, 23(6):807-21, dec 2004. ISSN 0167-9457. doi: 10.1016/j.humov.2004.08.023. URL http: //www.ncbi.nlm.nih.gov/pubmed/15664674.

[49] Y. Harada, J. Takahashi, D. Nenchev, and D. Sato. Limit cycle based walk of a powered 7DOF 3D biped with flat feet. 2010 IEEE/RSJ International Conference on Intelligent Robots and Systems, pages 3623-3628, oct 2010. doi: 10.1109/IROS.2010.5651546. URL http://ieeexplore.ieee.org/lpdocs/ epic03/wrapper . htm?arnumber $=5651546$.

[50] M. Hekmatfard, F. Farahmand, and I. Ebrahimi. Effects of prosthetic mass distribution on the spatiotemporal characteristics and knee kinematics of transfemoral amputee locomotion. Gait and Posture, 37(1):78-81, 2013. ISSN 09666362. doi: 10.1016/j.gaitpost.2012.06.010. URL http://dx.doi.org/10. $1016 / \mathrm{j}$.gaitpost . 2012.06 .010 .

[51] W. Herzog, B. M. Nigg, L. J. Read, and E. Olsson. Asymmetries in ground reaction force patterns in normal human gait. Medicine 8 Science in Sports \& Exercise, 21(1):110-114, 1989. ISSN 0195-9131. doi: 10.1249/ 00005768-198902000-00020.

[52] D. G. E. Hobbelen and M. Wisse. A disturbance rejection measure for limit cycle walkers: The gait sensitivity norm. IEEE Transactions on Robotics, 23 (6):1213-1224, 2007. ISSN 15523098. doi: 10.1109/TRO.2007.904908.

[53] A. L. Hof, R. M. van Bockel, T. Schoppen, and K. Postema. Control of lateral balance in walking. Experimental findings in normal subjects and above-knee amputees. Gait and Posture, 25(2):250-258, feb 2007. ISSN 09666362. doi: 10. 1016/j.gaitpost.2006.04.013. URL http://www.sciencedirect.com/science/ article/pii/S0966636206000580?via\{\%\}3ihub.

[54] J. H. Hollman, E. M. McDade, and R. C. Petersen. Normative spatiotemporal gait parameters in older adults. Gait and Posture, 34(1):111-118, 2011. ISSN 09666362. doi: 10.1016/j.gaitpost.2011.03.024. URL http://dx.doi.org/10. $1016 / j$.gaitpost . 2011.03 .024 .

[55] C. Honeycutt, J. Sushko, and K. B. Reed. Asymmetric passive dynamic walker. IEEE International Conference on Rehabilitation Robotics, pages 0-5, 2011. ISSN 19457898. doi: 10.1109/ICORR.2011.5975465.

[56] H. Hong, S. Kim, C. Kim, S. Lee, and S. Park. Spring-like gait mechanics observed during walking in both young and older adults. Journal of Biomechanics, 46(1):77-82, 2013. ISSN 00219290. doi: 10.1016/j.jbiomech.2012.10.003.

[57] G. R. B. Hurley, R. Mckenney, M. Robinson, M. Zadravec, M. R. Pierrynowski, and G. Hurley. The role of the contralateral limb in below-knee amputee gait. 
Prosthetics and Orthotics International, 14(1973):33-42, 1990. ISSN 0309-3646. doi: 10.3109/03093649009080314.

[58] Y. Ikemata, A. Sano, K. Yasuhara, and H. Fujimoto. Dynamic Effects of Arc Feet on the Leg Motion of Passive Walker. (1):2755-2760, 2009.

[59] E. Isakov, H. Burger, J. Krajnik, M. Gregoric, and C. Marincek. Influence of speed on gait parameters and on symmetry in trans-tibial amputees. Prosthetics and orthotics international, 20(3):153-158, 1996. ISSN 0309-3646. doi: 10.3109/ 03093649609164437.

[60] E. Isakov, O. Keren, and N. Benjuya. Trans-tibial amputee gait: time-distance parameters and EMG activity. Prosthetics and orthotics international, 24(3): 216-220, 2000. ISSN 0309-3646. doi: 10.1080/03093640008726550.

[61] Y. Jeon, Y. S. Park, and Y. Park. A study on stability of limit cycle walking model with feet: Parameter study. International Journal of Advanced Robotic Systems, 10:1-9, 2013. ISSN 17298806. doi: 10.5772/55162.

[62] T. S. Keller, A. M. Weisberger, J. L. Ray, S. S. Hasan, R. G. Shiavi, and D. M. Spengler. Relationship between vertical ground reaction force and speed during walking, slow jogging, and running. Clinical biomechanics (Bristol, Avon), 11 (5):253-259, jul 1996. ISSN 1879-1271. URL http://www.ncbi.nlm.nih.gov/ pubmed/11415629.

[63] J. Kim, C. H. Choi, and M. W. Spong. Passive dynamic walking with knee and fixed flat feet. In Conference Proceedings - IEEE International Conference on Systems, Man and Cybernetics, volume 00, pages 2744-2750, 2012. ISBN 9781467317146. doi: 10.1109/ICSMC.2012.6378163.

[64] S. Kim and S. Park. Leg stiffness increases with speed to modulate gait frequency and propulsion energy. Journal of Biomechanics, 44(7):1253-1258, 2011. ISSN 00219290. doi: 10.1016/j.jbiomech.2011.02.072.

[65] B. Kluitenberg, S. W. Bredeweg, S. Zijlstra, W. Zijlstra, and I. Buist. Comparison of vertical ground reaction forces during overground and treadmill running. A validation study. BMC Musculoskeletal Disorders, 13(November), 2012. ISSN 14712474. doi: 10.1186/1471-2474-13-235.

[66] R. Kram, A. Domingo, and D. P. Ferris. Effect of reduced gravity on the preferred walk-run transition speed. The Journal of experimental biology, 200 (Pt 4):821-826, 1997. ISSN 0022-0949.

[67] R. Kram, T. M. Griffin, J. Maxwell Donelan, and Y. Hui Chang. Force treadmill for measuring vertical and horizontal ground reaction forces. Journal of Applied Physiology, 7(9):764-769, 1998. ISSN 15461440. doi: 10.1016/j.jacr.2010.07.010. URL http://jap.physiology.org/content/85/ 2/764.full\{\#\}ref-list-1\{\%\}5Cnhttp://jap.physiology.org/content/ 
85/2/764\{\#\}Cited-by\{\%\}5Cnhttp://jap. physiology.org/content/85/2/ 764.full $\{\%\} 5$ Cnhttp://jap. physiology. org/.

[68] R. Kram, P. a. Willems, and T. P. Gosseye. A treadmill mounted force platform. Journal of Biomechanics, 22(12):1421-4, 2013. ISSN 00219290. doi: 10.1242/ bio.20136379. URL http://www.sciencedirect.com/science/article/pii/ 0021929089903321.

[69] P. A. Kramer and I. Sarton-Miller. The energetics of human walking: Is Froude number (Fr) useful for metabolic comparisons? Gait and Posture, 27(2):209215, 2008. ISSN 09666362. doi: 10.1016/j.gaitpost.2007.03.009.

[70] J. Kulkarni, C. Toole, R. Hirons, S. Wright, and J. Morris. Falls in patients with lower limb amputations: Prevalence and contributing factors. Physiotherapy, 82(2):130-136, 1996. ISSN 00319406. doi: 10.1016/S0031-9406(05)66968-4.

[71] J. Kulkarni, J. Adams, E. Thomas, and A. Silman. Association between amputation, arthritis and osteopenia in British male war veterans with major lower limb amputations. Clinical Rehabilitation, 12(3):274-279, 1998. ISSN 02692155. doi: 10.1191/026921598668452322.

[72] A. D. Kuo. A Simple Model of Bipedal Walking Predicts the Preferred SpeedStep Length Relationship. Journal of Biomechanical Engineering, 123(3):264, 2001. ISSN 01480731. doi: 10.1115/1.1372322. URL http://biomechanical. asmedigitalcollection . asme . org/article . aspx?articleid=1405720.

[73] A. D. Kuo. Energetics of Actively Powered Locomotion Using the Simplest Walking Model. Journal of Biomechanical Engineering, 124(1):113, sep 2002. ISSN 0148-0731. doi: 10.1115/1.1427703. URL http://biomechanical.asmedigitalcollection.asme.org/article. aspx?doi=10.1115/1.1427703.

[74] A. D. Kuo. The six determinants of gait and the inverted pendulum analogy: A dynamic walking perspective. Human Movement Science, 26(4):617-656, 2007. ISSN 01679457. doi: 10.1016/j.humov.2007.04.003. URL http://www. sciencedirect.com/science/article/pii/S0167945707000309.

[75] A. D. Kuo and J. M. Donelan. Dynamic Principles of Gait and Their Clinical Implications. Physical Therapy, 90(2):157-174, 2010. ISSN 0031-9023. doi: 10. 2522/ptj.20090125. URL https://academic.oup.com/ptj/article-lookup/ doi/10.2522/ptj. 20090125.

[76] A. D. Kuo, J. M. Donelan, and A. Ruina. Energetic consequences of walking like an inverted pendulum: step-to-step transitions. Exercise and sport sciences reviews, 33(2):88-97, 2005. ISSN 0091-6331. doi: 10.1097/ 00003677-200504000-00006. 
[77] M. Kwan and M. Hubbard. Optimal foot shape for a passive dynamic biped. Journal of theoretical biology, 248(2):331-9, sep 2007. ISSN 00225193. doi: 10.1016/j.jtbi.2007.05.008. URL http://www.ncbi.nlm.nih.gov/ pubmed/17570405.

[78] J. F. Lehmann, R. Price, R. Okumura, K. Questad, B. J. De Lateur, and A. Négretot. Mass and mass distribution of below-knee prostheses: Effect on gait efficacy and self-selected walking speed. Archives of Physical Medicine and Rehabilitation, 79(2):162-168, 1998. ISSN 00039993. doi: 10.1016/S0003-9993(98)90293-3.

[79] Q. Li and X. S. Yang. New walking dynamics in the simplest passive bipedal walking model. Applied Mathematical Modelling, 36(11):5262-5271, 2012. ISSN 0307904X. doi: 10.1016/j.apm.2011.12.049. URL http://dx.doi.org/10. 1016/j.apm.2011.12.049.

[80] S. W. Lipfert. Kinematic and Dynamic Similarities between Walking and Running. City, (April):1-74, 2010.

[81] S. W. Lipfert, M. Günther, D. Renjewski, S. Grimmer, and A. Seyfarth. A model-experiment comparison of system dynamics for human walking and running. Journal of Theoretical Biology, 292:11-17, 2012. ISSN 00225193. doi: 10.1016/j.jtbi.2011.09.021. URL http://www.sciencedirect.com/science/ article/pii/S0022519311004851.

[82] S. W. Lipfert, M. Günther, D. Renjewski, and A. Seyfarth. Impulsive ankle push-off powers leg swing in human walking. The Journal of Experimental Biology, 217(Pt 8):1218-28, 2014. ISSN 1477-9145. doi: 10.1242/jeb.097345.

[83] M. Q. Liu, F. C. Anderson, M. G. Pandy, and S. L. Delp. Muscles that support the body also modulate forward progression during walking. Journal of biomechanics, 39(14):2623-30, jan 2006. ISSN 0021-9290. doi: 10.1016/j.jbiomech. 2005.08.017. URL http://www.ncbi.nlm.nih.gov/pubmed/16216251.

[84] M. Q. Liu, F. C. Anderson, M. H. Schwartz, and S. L. Delp. Muscle contributions to support and progression over a range of walking speeds. Journal of biomechanics, 41(15):3243-52, nov 2008. ISSN 0021-9290. doi: 10. 1016/j.jbiomech.2008.07.031. URL http://www.ncbi.nlm.nih.gov/pubmed/ 18822415.

[85] L. L. Long and M. Srinivasan. Walking, running, and resting under time, distance, and average speed constraints: optimality of walk-run-rest mixtures. Journal of The Royal Society Interface, 10:20120980, 2013. ISSN 1742-5662. doi: 10.1098/rsif.2012.0980.

[86] M. E. Lund, M. de Zee, M. S. Andersen, and J. Rasmussen. On validation of multibody musculoskeletal models. Proceedings of the Institution of Mechanical 
Engineers, Part H: Journal of Engineering in Medicine, 226(2):82-94, 2012. ISSN 09544119. doi: 10.1177/0954411911431516.

[87] M. E. Lund, M. S. Andersen, M. de Zee, and J. Rasmussen. Scaling of musculoskeletal models from static and dynamic trials. International Biomechanics, 2(1):1-11, 2015. ISSN 23335432. doi: 10.1080/23335432.2014.993706. URL http://dx.doi.org/10.1080/23335432.2014.993706.

[88] P. Mahmoodi. Optimisation of Bipedal Walking Motion with Unbalanced Masses. PhD thesis, Swansea University, 2013.

[89] P. Mahmoodi, R. S. Ransing, and M. I. Friswell. Modelling the effect of 'heel to toe' roll-over contact on the walking dynamics of passive biped robots. Applied Mathematical Modelling, 37(12-13):7352-7373, jul 2013. ISSN 0307904X. doi: 10.1016/j.apm.2013.02.048. URL http://linkinghub.elsevier.com/ retrieve/pii/S0307904X13001558.

[90] P. Mahmoodi, R. S. Ransing, and M. I. Friswell. A novel mathematical formulation for predicting symmetric passive bipedal walking motion with unbalanced masses. Applied Mathematical Modelling, 40(5-6):3895-3906, mar 2016. ISSN 0307904X. doi: 10.1016/j.apm.2015.10.051. URL http://www. sciencedirect.com/science/article/pii/S0307904X15007155.

[91] S. J. Mattes, P. E. Martin, and T. D. Royer. Walking symmetry and energy cost in persons with unilateral transtibial amputations: Matching prosthetic and intact limb inertial properties. Archives of Physical Medicine and Rehabilitation, 81(5):561-568, 2000. ISSN 00039993. doi: 10.1053/mr.2000.3851.

[92] S. J. Mattes, P. E. Martin, and T. D. Royer. Walking Symmetry and Energy Cost in Persons With Unilateral Transtibial Amputations: Matching Prosthetic and Intact Limb Inertial Properties. Arch Phys Med Rehabil, 81:561-8, 2000. doi: $10.1053 /$ nu.2000.385.

[93] H. M. Maus, S. Lipfert, M. Gross, J. Rummel, and A. Seyfarth. Upright human gait did not provide a major mechanical challenge for our ancestors. Nature Communications, 1(6):1-6, 2010. ISSN 2041-1723. doi: 10.1038/ncomms1073. URL http://www . nature.com/doifinder/10.1038/ncomms1073.

[94] T. McGeer. Passive Dynamic Walking. The International Journal of Robotics Research, 9(2):62-82, apr 1990. ISSN 0278-3649. doi: 10.1177/ 027836499000900206. URL http://ijr.sagepub.com/cgi/doi/10.1177/ 027836499000900206.

[95] A. Merker, J. Rummel, and A. Seyfarth. Stable walking with asymmetric legs. Bioinspiration \&3 biomimetics, 6(4):045004, 2011. ISSN 1748-3190. doi: 10.1088/1748-3182/6/4/045004. URL http://iopscience.iop.org/ article/10.1088/1748-3182/6/4/045004. 
[96] W. C. Miller, A. B. Deathe, M. Speechley, and J. Koval. The influence of falling, fear of falling, and balance confidence on prosthetic mobility and social activity among individuals with a lower extremity amputation. Archives of Physical Medicine and Rehabilitation, 82(9):1238-1244, 2001. ISSN 00039993. doi: 10. 1053/apmr.2001.25079. URL http://linkinghub.elsevier.com/retrieve/ pii/S0003999301150264.

[97] A. E. Minetti and F. Saibene. Mechanical work rate minimization and freely chosen stride frequency of human walking: A mathematical model. J. Exp. Biol, 170:19-34, 1992. ISSN 0022-0949.

[98] N. Mizuno, T. Aoyama, A. Nakajima, T. Kasahara, and K. Takami. Functional evaluation by gait analysis of various ankle-foot assemblies used by below-knee amputees. Prosthetics and orthotics international, 16(3):174-182, 1992. ISSN 0309-3646. doi: 10.3109/03093649209164337.

[99] S. Mochon and T. A. McMahon. Ballistic walking: an improved model. Mathematical Biosciences, 52(3-4):241-260, jan 1980. ISSN 00255564. doi: 10.1016/0025-5564(80)90070-X. URL http://www.sciencedirect. com/science/article/pii/002192908090007X?via\{\%\}3ihubhttp: //www.sciencedirect.com/science/article/pii/002192908090007X.

[100] J. K. Moore, S. K. Hnat, and A. J. van den Bogert. An elaborate data set on human gait and the effect of mechanical perturbations. PeerJ, 3(January): e918, 2015. ISSN 2167-8359. doi: 10.7717/peerj.918. URL https://peerj . com/articles/918.

[101] R. R. Neptune, F. E. Zajac, and S. A. Kautz. Muscle mechanical work requirements during normal walking: the energetic cost of raising the body's center-of-mass is significant. Journal of biomechanics, 37(6):817-25, jun 2004. ISSN 0021-9290. doi: 10.1016/j.jbiomech.2003.11.001. URL http://www .ncbi. nlm.nih.gov/pubmed/15111069.

[102] R. R. Neptune, K. Sasaki, and S. A. Kautz. The effect of walking speed on muscle function and mechanical energetics. Gait $\&$ Posture, 28(1):135-143, jul 2008. ISSN 09666362. doi: 10.1016/j.gaitpost.2007.11.004. URL http: //linkinghub.elsevier.com/retrieve/pii/S096663620700269X.

[103] L. Nolan, A. Wit, K. Dudziñski, A. Lees, M. Lake, and M. Wychowañski. Adjustments in gait symmetry with walking speed in trans-femoral and transtibial amputees. Gait \& Posture, 17(2):142-151, apr 2003. ISSN 0966-6362. doi: 10.1016/S0966-6362(02)00066-8. URL https://www.sciencedirect. com/science/article/pii/S0966636202000668.

[104] D. C. Norvell, J. M. Czerniecki, G. E. Reiber, C. Maynard, J. A. Pecoraro, and N. S. Weiss. The prevalence of knee pain and symptomatic knee osteoarthritis among veteran traumatic amputees and nonamputees. Archives of Physical 
Medicine and Rehabilitation, 86(3):487-493, mar 2005. ISSN 00039993. doi: 10. 1016/j.apmr.2004.04.034. URL https://www.sciencedirect.com/science/ article/pii/S000399930400629X?via=ihub.

[105] H. G. A. A. M. R. L. W.-P. A. F. B. Ohio. Anthropometry and mass distribution for human analogues. volume 1. military male aviators. 1988.

[106] K. M. Olesnavage and A. G. Winter. Design and Qualitative Testing of a Prosthetic Foot With Rotational Ankle and Metatarsal Joints to Mimic Physiological Roll-Over Shape. In Volume 5A: 39th Mechanisms and Robotics Conference, page V05AT08A035. ASME, aug 2015. ISBN 978-0-7918-5712-0. doi: 10.1115/ DETC2015-46518. URL http://proceedings.asmedigitalcollection. asme .org/proceeding. aspx?doi=10.1115/DETC2015-46518.

[107] J. Perry. Gait Analysis: Normal and Pathological Function, volume 12. 2010. ISBN ISBN 978-1-55642-766-4. doi: 10.1001.

[108] C. Powers, L. Torburn, J. Perry, and E. Ayyappa. Influence of prosthetic foot design on sound limb loading in adults with unilateral below-knee amputations. Archives of physical medicine and rehabilitation, 75(7):825-829, 1994. ISSN 00039993. doi: 10.5555/uri:pii:0003999394901465.

[109] M. Rabuffetti and G. Baroni. Validation protocol of models for centre of mass estimation. Journal of Biomechanics, 32(6):609-613, 1999. ISSN 00219290. doi: 10.1016/S0021-9290(99)00040-8.

[110] H. J. Ralston. Energy-speed relation and optimal speed during level walking. Internationale Zeitschrift für angewandte Physiologie einschließlich Arbeitsphysiologie, 17(4):277-283, 1958.

[111] F. Rasouli, M. Naraghi, and A. Safa. Asymmetric gait analysis based on passive dynamic walking theory. 4th RSI International Conference on Robotics and Mechatronics, ICRoM 2016, pages 361-366, 2017. doi: 10.1109/ICRoM.2016. 7886765 .

[112] A. Ruina. Nonholonomic stability aspects of piecewise holonomic systems. Reports on Mathematical Physics, 42(1-2):91-100, aug 1998. ISSN 00344877. doi: http://dx.doi.org/10.1016/S0034-4877(98)80006-2. URL https://www . sciencedirect.com/science/article/pii/S0034487798800062.

[113] J. Rummel, Y. Blum, H. M. Maus, C. Rode, and A. Seyfarth. Stable and robust walking with compliant legs. Proceedings - IEEE International Conference on Robotics and Automation, pages 5250-5255, 2010. ISSN 10504729. doi: 10. 1109/ROBOT.2010.5509500.

[114] E. Russell Esposito, J. M. Aldridge Whitehead, and J. M. Wilken. Sound limb loading in individuals with unilateral transfemoral amputation across a range of walking velocities. Clinical Biomechanics, 30 
(10):1049-1055, dec 2015. ISSN 18791271. doi: 10.1016/j.clinbiomech. 2015.09.008. URL https://www.sciencedirect.com/science/article/pii/ S0268003315002491?\{_\}rdoc=1.

[115] H. Sadeghi, P. Allard, and M. Duhaime. Functional gait asymmetry in ablebodied subjects, 1997. ISSN 01679457.

[116] H. Sadeghi, P. Allard, F. Prince, and H. Labelle. Symmetry and limb dominance in able-bodied gait: A review, sep 2000. ISSN 09666362. URL http://www. sciencedirect.com/science/article/pii/S0966636200000709.

[117] G. S. Sawicki, C. L. Lewis, and D. P. Ferris. It pays to have springs in your step. Exercise and sport sciences reviews, 37(3):1-19, 2013. doi: 10.1097/JES. 0b013e31819c2df6.It.

[118] M. Schaarschmidt, S. W. Lipfert, C. Meier-Gratz, H. C. Scholle, and A. Seyfarth. Functional gait asymmetry of unilateral transfemoral amputees. Human Movement Science, 31(4):907-917, 2012. ISSN 01679457. doi: 10.1016/j.humov. 2011.09.004. URL http://dx.doi.org/10.1016/j.humov.2011.09.004.

[119] A. Schüler. Three Methods To Determine Mass Characteristics of Human Body Segments. ISBS-Conference ..., pages 224-227, 2014. URL https://ojs.ub. uni-konstanz.de/cpa/article/view/5925.

[120] A. L. Schwab and M. Wisse. Basin of Attraction of the Simplest Walking Model. In ASME Design Engineering Technical Conference 6, pages 531-539, 2001. ISBN 1549-9596. doi: 10.1021/ci2001988. URL http://audiophile. tam. cornell. edu/\{ $\}$ als93/Publications/vib21363.pdf $\{\%\} 5$ Cnhttp: //bicycle.tudelft.nl/schwab/Publications/vib21363.pdf.

[121] R. W. Selles, J. B. J. Bussmann, R. C. Wagenaar, and H. J. Stam. Effects of prosthetic mass and mass distribution on kinematics and energetics of prosthetic gait: A systematic review. Archives of Physical Medicine and Rehabilitation, 80(12):1593-1599, 1999. ISSN 00039993. doi: 10.1016/S0003-9993(99)90336-2.

[122] R. W. Selles, J. B. Bussmann, L. M. Klip, B. Speet, a. J. Van Soest, and H. J. Stam. Adaptations to mass perturbations in transtibial amputees: Kinetic or kinematic invariance? Archives of Physical Medicine and Rehabilitation, 85 (12):2046-2052, 2004. ISSN 00039993. doi: 10.1016/j.apmr.2003.10.013.

[123] R. W. Selles, J. B. J. Bussmann, A. J. K. Van Soest, and H. J. Stam. The effect of prosthetic mass properties on the gait of transtibial amputees - A mathematical model. Disability and Rehabilitation, 26(12):694-704, 2004. ISSN 09638288. doi: 10.1080/09638280410001704296.

[124] A. Seyfarth, H. Geyer, S. Lipfert, J. Rummel, Y. Minekawa, and F. Iida. Running and walking with compliant legs. Modeling, Simulation and Optimization, COSMOS 18., 18(January):43-51, 2013. doi: 10.1007/978-3-642-36368-9. URL http://link. springer.com/10.1007/978-3-642-36368-9. 
[125] N. H. Shah and M. A. Yeolekar. Influence of Slope Angle on the Walking of Passive Dynamic Biped Robot. (March):456-465, 2015.

[126] M. A. Sharbafi and A. Seyfarth. How locomotion sub-functions can control walking at different speeds? Journal of Biomechanics, 53(January):163-170, 2017. ISSN 18732380. doi: 10.1016/j.jbiomech.2017.01.018. URL http://dx . doi.org/10.1016/j.jbiomech.2017.01.018.

[127] M. A. Sharbafi, A. M. N. Rashty, C. Rode, and A. Seyfarth. Reconstruction of human swing leg motion with passive biarticular muscle models. Human Movement Science, 52:96-107, 2017. ISSN 18727646. doi: 10.1016/j.humov. 2017.01.008. URL http://www.sciencedirect.com/science/article/pii/ S0167945717300313.

[128] J. M. Shippen and B. May. Calculation of muscle loading and joint contact forces during the rock step in Irish dance. Journal of dance medicine 8 science, 14(1):11-18, 2010. ISSN 1089-313X. doi: 10.1017/S000748530002229X.

[129] E. B. Simonsen. Contributions to the understanding of gait control. Danish Medical Journal, 61(4), 2014. ISSN 22451919.

[130] I. Singh. Functional asymmetry in the lower limbs. Cells Tissues Organs, 77 (1):131-138, 1970. ISSN 14226421. doi: 10.1159/000143535.

[131] J. D. Smith and P. E. Martin. Walking patterns change rapidly following asymmetrical lower extremity loading. Human Movement Science, 26(3):412-425, 2007. ISSN 01679457. doi: 10.1016/j.humov.2006.12.001.

[132] J. D. Smith and P. E. Martin. Effects of prosthetic mass distribution on metabolic costs and walking symmetry. Journal of Applied Biomechanics, 29 (3):317-328, 2013. ISSN 10658483.

[133] R. D. Snyder, C. M. Powers, C. Fontaine, and J. Perry. The effect of five prosthetic feet on the gait and loading of the sound limb in dysvascular belowknee amputees. Journal of rehabilitation research and development, 32(4):309315, 1995. ISSN 0748-7711.

[134] H. Song, H. Park, and S. Park. A springy pendulum could describe the swing leg kinetics of human walking. Journal of Biomechanics, 49(9):1504-1509, 2016. ISSN 00219290. doi: 10.1016/j.jbiomech.2016.03.018.

[135] K. M. Steele, A. Seth, J. L. Hicks, M. S. Schwartz, and S. L. Delp. Muscle contributions to support and progression during single-limb stance in crouch gait. Journal of biomechanics, 43(11):2099-105, aug 2010. ISSN 1873-2380. doi: 10.1016/j.jbiomech.2010.04.003. URL https://www.ncbi.nlm.nih.gov/ pubmed/20493489. 
[136] T. Stöckel, R. Jacksteit, M. Behrens, R. Skripitz, R. Bader, and A. MauMoeller. The mental representation of the human gait in young and older adults. Frontiers in Psychology, 6(July):1-10, 2015. ISSN 1664-1078. doi: 10. 3389/fpsyg.2015.00943. URL http://journal.frontiersin.org/Article/ 10.3389/fpsyg. 2015.00943/abstract.

[137] J. Sushko, C. Honeycutt, and K. B. Reed. Prosthesis design based on an asymmetric passive dynamic walker. In Proceedings of the IEEE RAS and EMBS International Conference on Biomedical Robotics and Biomechatronics, pages 1116-1121, 2012. ISBN 9781457711992. doi: 10.1109/BioRob.2012.6290293. URL http://ieeexplore.ieee.org/document/6290293/.

[138] J. A. Thompson, A. M. W. Chaudhari, L. C. Schmitt, T. M. Best, and R. A. Siston. Gluteus maximus and soleus compensate for simulated quadriceps atrophy and activation failure during walking. Journal of biomechanics, 46(13): 2165-72, sep 2013. ISSN 1873-2380. doi: 10.1016/j.jbiomech.2013.06.033. URL http://www.ncbi.nlm.nih.gov/pubmed/23915576.

[139] K. B. Trifonov and S. Hashimoto. Active knee-release mechanism for passivedynamic walking machines and walking cycle research. In 2008 IEEE/RSJ International Conference on Intelligent Robots and Systems, IROS, pages 179184, 2008. ISBN 9781424420582. doi: 10.1109/IROS.2008.4650927.

[140] C. Tudor-Locke, W. D. Johnson, and P. T. Katzmarzyk. Accelerometerdetermined steps per day in US children and youth. Medicine and Science in Sports and Exercise, 42(12):2244-2250, 2010. ISSN 01959131. doi: 10.1249/MSS.0b013e3181e32d7f.

[141] K. A. Valenzuela, S. K. Lynn, L. R. Mikelson, G. J. Noffal, and D. A. Judelson. Effect of acute alterations in foot strike patterns during running on sagittal plane lower limb kinematics and kinetics. Journal of Sports Science and Medicine, 14(1):225-232, 2014. ISSN 13032968.

[142] N. Vanicek, S. Strike, L. McNaughton, and R. Polman. Gait patterns in transtibial amputee fallers vs. non-fallers: Biomechanical differences during level walking. Gait and Posture, 29(3):415-420, apr 2009. ISSN 09666362. doi: 10.1016/j.gaitpost.2008.10.062. URL https://www.ncbi.nlm.nih.gov/ pubmed/19071021.

[143] H. R. Vejdani, A. Wu, H. Geyer, and J. W. Hurst. Touch-down angle control for spring-mass walking. Robotics and Automation (ICRA), 2015 IEEE International Conference on, pages 5101-5106, 2015. ISSN 10504729. doi: 10.1109/ICRA.2015.7139909.

[144] D. W. Wagner, V. Stepanyan, J. M. Shippen, M. S. Demers, R. S. Gibbons, B. J. Andrews, G. H. Creasey, and G. S. Beaupre. Consistency among musculoskeletal models: Caveat utilitor. Annals of Biomedical Engineering, 41(8):1787-1799, 2013. ISSN 00906964. doi: 10.1007/s10439-013-0843-1. 
[145] C. C. Wang and A. H. Hansen. Response of able-bodied persons to changes in shoe rocker radius during walking: changes in ankle kinematics to maintain a consistent roll-over shape. Journal of biomechanics, 43(12):2288-93, aug 2010. ISSN 1873-2380. doi: 10.1016/j.jbiomech.2010.04.036. URL https://www . ncbi.nlm.nih.gov/pubmed/20483413.

[146] Q. Wang, L. Wang, Y. Huang, J. Zhu, and W. Chen. Three-dimensional quasi-passive dynamic bipedal walking with flat feet and compliant ankles. Proceedings of the $48 \mathrm{~h}$ IEEE Conference on Decision and Control (CDC) held jointly with 2009 28th Chinese Control Conference, pages 8200-8205, dec 2009. doi: 10.1109/CDC.2009.5399656. URL http://ieeexplore.ieee.org/ lpdocs/epic03/wrapper .htm?arnumber $=5399656$.

[147] B. R. Whittington and D. G. Thelen. A Simple Mass-Spring Model with Roller Feet can induce the Ground Reactions Observed in Human Walking. J Biomech Eng., 131(1):1-20, 2009. doi: 10.1115/1.3005147.A.

[148] M. Whittle. Gait analysis : an introduction. Butterworth-Heinemann, 2007. ISBN 9780750688833.

[149] M. Wisse, D. Hobbelen, R. Rotteveel, S. Anderson, and G. Zeglin. Ankle springs instead of arc-shaped feet for passive dynamic walkers. 2006 6th IEEE-RAS International Conference on Humanoid Robots, pages 110-116, dec 2006. doi: 10.1109/ICHR.2006.321371. URL http://ieeexplore.ieee.org/ lpdocs /epic03/wrapper .htm?arnumber $=4115588$.

[150] V. Zatsiorsky and V. N. Seluyanov. Mass-inertial characteristics of the segments of the human body and their relationship with anthropometric landmarks. Voprosy Antropologii, 62:91-103, 1979.

[151] K. E. Zelik, S. H. Collins, P. G. Adamczyk, A. D. Segal, G. K. Klute, D. C. Morgenroth, M. E. Hahn, M. S. Orendurff, J. M. Czerniecki, and A. D. Kuo. Systematic variation of prosthetic foot spring affects center-of-mass mechanics and metabolic cost during walking. IEEE Transactions on Neural Systems and Rehabilitation Engineering, 19(4):411-419, 2011. ISSN 15344320. doi: 10.1109/ TNSRE.2011.2159018.

[152] K. E. Zelik, T. W. P. Huang, P. G. Adamczyk, and A. D. Kuo. The role of series ankle elasticity in bipedal walking. Journal of Theoretical Biology, 346: 75-85, 2014. ISSN 00225193. doi: 10.1016/j.jtbi.2013.12.014.

[153] K. E. Zelik, K. Z. Takahashi, and G. S. Sawicki. Six degree-of-freedom analysis of hip, knee, ankle and foot provides updated understanding of biomechanical work during human walking. Journal of Experimental Biology, 218 (6):876-886, 2015. ISSN 0022-0949. doi: 10.1242/jeb.115451. URL http: //jeb.biologists.org/cgi/doi/10.1242/jeb.115451. 
[154] L. Zhenze, T. Yantao, Z. Peijie, and Z. Changjiu. The analysis on bifurcation and chaos in the compass-gait biped. 2007 IEEE International Conference on Robotics and Biomimetics, ROBIO, (2006):972-977, 2007. doi: 10.1109/ ROBIO.2007.4522295. 